Pacific Northwest

National Laboratory

Operated by Battelle for the

U.S. Department of Energy

\title{
Analysis of Induced Gas Released During Retrieval of Hanford Double-Shell Tank Waste
}

\author{
BE Wells \\ JM Cuta \\ SA Hartley \\ LA Mahoney \\ PA Meyer \\ CW Stewart
}

March 2002

Prepared for the U.S. Department of Energy under Contract DE-AC06-76RL01830 


\section{DISCLAIMER}

This report was prepared as an account of work sponsored by an agency of the United States Government. Neither the United States Government nor any agency thereof, nor Battelle Memorial Institute nor any of their employees makes any warranty, express or implied, or assumes any legal liability or responsibility for the accuracy, completeness, or usefulness of any information, apparatus, product, or process disclosed or represents that its use would not infringe privately owned rights. Reference herein to any specific commercial product, process, or service by trade name, trademark, manufacturer, or otherwise does not necessarily constitute or imply its endorsement, recommendation, or favoring by the United States Government or any agency thereof, or Battelle Memorial Institute. The views and opinions of authors expressed herein do not necessarily state or reflect those of the United States Government or any agency thereof.

\section{PACIFIC NORTHWEST NATIONAL LABORATORY \\ operated by \\ BATTELLE \\ for the \\ UNITED STATES DEPARTMENT OF ENERGY \\ under Contract DE-AC06-76RL01830}

Printed in the United States of America

Available to DOE and DOE contractors from the

Office of Scientific and Technical Information,

P.O. Box 62, Oak Ridge, TN 37831-0062;

ph: (865) 576-8401

fax: (865) $576-5728$

email: reports@adonis.osti.gov

Available to the public from the National Technical Information Service,

U.S. Department of Commerce, 5285 Port Royal Rd., Springfield, VA 22161

ph: (800) 553-6847

fax: (703) 605-6900

email: orders@ntis.fedworld.gov

online ordering: http://www.ntis.gov/ordering.htm

This document was printed on recycled paper. 


\section{Analysis of Induced Gas Releases During Retrieval of Hanford Double-Shell Tank Waste}

BE Wells

JM Cuta

SA Hartley

LA Mahoney

PA Meyer

CW Stewart

March 2002

Prepared for

the U.S. Department of Energy

under contract DE-AC06-76RL01830

Pacific Northwest National Laboratory

Richland, WA 99352 


\begin{abstract}
Radioactive waste is currently scheduled to be retrieved from Hanford double-shell tanks AN-103, AN-104, AN-105 and AW-101 and transferred to the vitrification plant beginning about 2009. Retrieval may involve decanting the supernatant liquid and/or mixing the waste with jet pumps. In these four tanks, which contain relatively large volumes of retained gas, both of these operations are expected to induce buoyant displacement gas releases that can potentially raise the tank headspace hydrogen concentration to very near the lower flammability limit. This report describes the theory and detailed physical models for both the supernate decant and jet mixing processes and presents the results from applying the models to these operations in the four tanks. The technical bases for input parameter distributions are elucidated.
\end{abstract}




\section{Executive Summary}

Retrieval of Hanford radioactive tank waste from four double-shell tanks (DSTs), AN-103, AN-104, AN-105, and AW-101, to the vitrification plant is planned for 2009 to 2019. Retrieval will involve decanting the supernatant liquid and/or mixing the waste with two large jet mixer pumps. All four tanks retain sufficient gas to reach or exceed the lower flammability limit (LFL) if a large fraction of the retained gas volume were released suddenly. They also exhibit spontaneous buoyant displacement (BD) gas release events (GREs).

Given this relatively large volume of retained gas and a history of relatively large spontaneous gas releases, retrieval operations need to be planned carefully to ensure they can be performed safely. The large mixer pumps planned to be installed to aid retrieval can quickly mobilize most of the waste in the tank. Upon pump startup, they could release a large fraction of a tank's stored gas in a short time. Decanting the supernatant liquid can be expected to induce a series of BDGREs by reducing the hydrostatic pressure and causing the stored gas to expand.

Detailed models of the gas release behavior during both supernate decant and initial mixer pump operation have been developed that include the dominant phenomena and important mechanisms that can potentially exacerbate gas releases. This report describes the development of the models, the selection of inputs, and the results of simulations of supernate decant and initial waste mixing in the four tanks.

\section{Gas Releases Induced by Supernate Decanting}

Supernate is to be decanted by a transfer pump installed just above the top of the sediment layer. As the liquid is removed, the hydrostatic pressure on the gas retained in the sediment layer decreases, causing the trapped gas bubbles to expand, which increases the gas volume fraction. Portions, or "gobs," of the sediment with a sufficiently high initial gas volume fraction will become buoyant and undergo BDGREs. Once buoyant, gobs induced to rise by the decant are assumed to behave like those participating in spontaneous gas releases.

The initial gob size and gas fraction distributions are determined by tank historical behavior and measured data, as is the gas release rate when a BDGRE occurs during decant. Sediment suspended during prior BDGREs increases the density of the liquid, which increases the buoyancy of the gobs relative to the liquid and hence further promotes BDGREs.

As the supernate depth decreases, the tank headspace increases so the same gas release volume will produce a progressively lower hydrogen concentration. At some point, the supernate depth will be insufficient to provide the energy required to yield the waste, and gas releases will effectively cease. Thus the highest hydrogen concentrations resulting from induced BDGREs tends to occur early in the process.

The model was applied using Monte Carlo simulation. A large number of model runs were performed with different sets of input to correctly portray the combined uncertainty of the input parameters in the results. The input sets for each run were selected from probability distributions 
for each parameter such that the entire uncertainty range was covered. Probability distributions representing the results were then derived from the set of outputs from all the runs. The results were most sensitive to the liquid and sediment densities, which determine the gas volume fraction at neutral buoyancy, and the gas fraction and size of the initial BDGRE.

The base case simulation comparing the gas release behavior of the four tanks used a decant rate of $200 \mathrm{gpm}$. Three additional cases were studied: a 30-gpm decant rate, a control strategy to pause the decant when the hydrogen concentration exceeded the action level of $6,250 \mathrm{ppm}$ and then restart at $500 \mathrm{ppm}$, and a second control strategy to backfill with water at the same rate as the decant to limit the decrease in hydrostatic pressure.

The results of the simulations in AN-105 are summarized as follows:

- The peak hydrogen concentration at the $95 \%$ confidence level remained at or below $8,300 \mathrm{ppm}$ in all cases (ranged from a maximum of 8,300 $\mathrm{ppm}$ in the base case to 7,600 ppm for the water backfill control strategy).

- The median value of the fraction of retained gas in the sediment layer released during decant was only 0.09 , and 0.22 or less at the $95 \%$ confidence level.

- Reducing the decant rate to $30 \mathrm{gpm}$ had essentially no effect on the peak hydrogen concentration or any other output parameter except to increase the total decant time in proportion to the lower rate.

- The stop-start control strategy had a minimal effect on the peak hydrogen concentration but extended the time for decant by about a factor of 3 .

- Backfilling the tank with water did not significantly change the hydrogen concentration at the $95 \%$ confidence level but prevented any BDGREs in over $76 \%$ of the cases.

The simulations for Tanks AN-103, AN-104, and AW-101 exhibited induced BDGRE behavior similar to that of AN-105. The peak hydrogen concentration distribution for AN-104 was similar to AN-105 because its slightly larger retained gas volume was balanced by a similarly larger headspace. Because of its much lower retained gas volume, the peak hydrogen concentrations in AW-101 remained well below the action level of 6,250 ppm for all cases. Because AN-103 contains more than twice the retained gas volume of AN-105 and the initial gas volume fraction is more likely to be closer to neutral buoyancy, its peak hydrogen concentrations at the $95 \%$ confidence level were much larger, $21,100 \mathrm{ppm}$, exceeding $50 \%$ of the LFL (approximately 20,000 ppm).

Though these predictions indicate that AN-103 has a greater potential hazard than the other tanks, the results are much more uncertain. There have been too few spontaneous BDGREs in this tank from which to derive statistically valid input distributions. It may be that decantinduced gas releases in this tank will follow its historical trend in a series of small releases that do not challenge flammability. However, the high uncertainty should engender a corresponding level of caution in planning its retrieval. 


\section{Gas Releases Induced by Initial Mixer Pump Operation}

Jet mixing is a mechanical disturbance that mobilizes a sediment by creating hydrodynamic shear and pressure forces that exceed the strength of the material. This disruption of sediment releases retained gas bubbles that eventually enter the tank headspace. Thus gas release is expected from the volume of waste actually disrupted. A powerful mixer pump may potentially affect a large waste volume at a relatively high rate, which, by design, suspends a large fraction of the mobilized solids in the liquid layer. As described above, this increases the density of the supernate, reducing the neutral buoyancy gas fraction and potentially inducing BDGREs in gobs that were not buoyant when mixing began.

The model developed to represent this process treats gas releases from both the direct disturbance of the mixer pump and the subsequent induced BDGREs. It consists of two independent parts: the first estimates the volume of waste actually mobilized by a mixer pump of known characteristics running at a given speed and duration; the second determines the headspace hydrogen concentration resulting from a specified schedule of pump runs, each of which mobilizes a given volume of waste. The first model is built on jet theory, and the second is a direct adaptation of the detailed supernate decant model described above, with relatively minor modifications.

It is assumed in the mixing model that the flow from the pump nozzles behaves as a turbulent jet impinging on and slowly penetrating a wall of weak solid material. The net hydraulic power of the jet deposited on the waste at the penetration zone is set equal to the power required to yield the solid matrix. Jet theory applied to this energy balance shows that the maximum volume of waste disturbed is proportional to the quantity $\left(\mathrm{u}_{0} \mathrm{~d}_{0}\right)^{3}$, where $\mathrm{u}_{0}$ and $\mathrm{d}_{0}$ are the initial jet velocity and nozzle diameter, respectively, while the time required to achieve this disturbance is proportional to $\mathrm{u}_{0} \mathrm{~d}_{0}$. The model was used to extrapolate gas release data from initial mixing tests in SY-101 to degassing AN-105 with much larger mixer pumps. The results indicate that a 26minute mixer pump run at $1200 \mathrm{rpm}$ (maximum speed) would theoretically mobilize $5 \%$ of the sediment volume in AN-105. A 76-minute run at the same speed mobilizes about $20 \%$ of the sediment.

The gas release model results for AN-105 indicate that $5 \%$ of the sediment can be mobilized per pump run on a schedule of one run per day and the headspace maintained well below the LFL. The median of the peak hydrogen concentration for this schedule is $7,500 \mathrm{ppm}$ with $18,100 \mathrm{ppm}$ at the $95 \%$ confidence level. Raising the disturbance to $20 \%$ of the sediment layer per run per day exceeds $50 \%$ of the LFL with 28,400 ppm of hydrogen at the $95 \%$ confidence level.

AN-104, with a larger headspace than AN-105, has a 95\% confidence level at 18,500 ppm for runs disturbing $20 \%$ of the sediment layer every 24 hours. The low gas volume of AW-101 allowed for runs disturbing $20 \%$ of the sediment layer on a schedule of three times per day. The median peak hydrogen concentration is $6,900 \mathrm{ppm}$, and the $95 \%$ confidence level is $8,800 \mathrm{ppm}$. The large headspace of AN-103 is overwhelmed by the high gas content, with pump runs disturbing only $5 \%$ of the sediment layer every 24 hours, producing a median peak hydrogen concentration of $15,300 \mathrm{ppm}$ and a $95 \%$ confidence level of $26,000 \mathrm{ppm}$. 


\section{Contents}

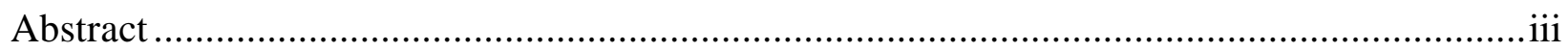

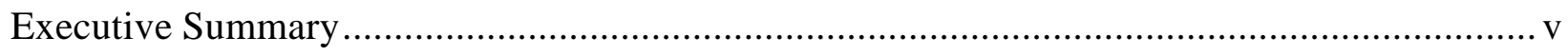

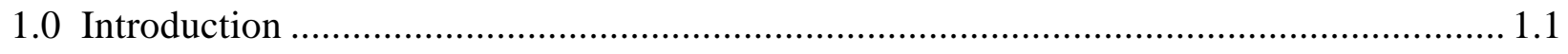

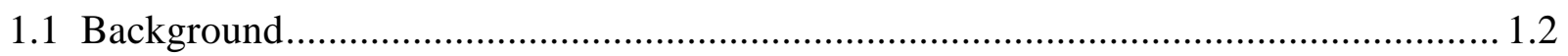

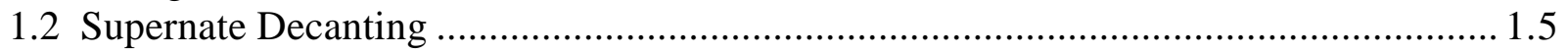

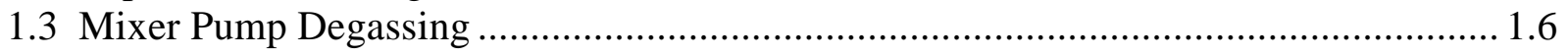

1.4 Improving the Models and Predictions …………...................................................... 1.8

2.0 Phenomena That Could Amplify Gas Releases.............................................................. 2.1

2.1 Phenomena Active During Supernate Decanting ....................................................... 2.1

2.1.1 Mechanisms Making More Gobs Buoyant at the Same Time ................................... 2.1

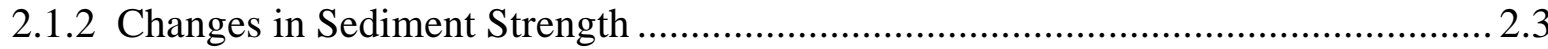

2.1.3 Changes in Void Profile Shape Due to Depressurization ........................................ 2.4

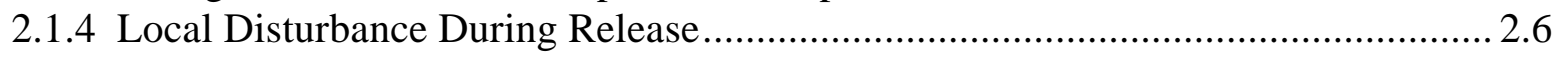

2.1.5 Global Disturbance During Release of First Gob .................................................. 2.7

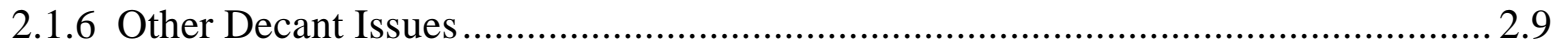

2.2 Phenomena Affecting Initial Mixer Pump Operation ................................................. 2.11

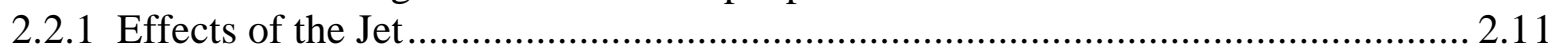

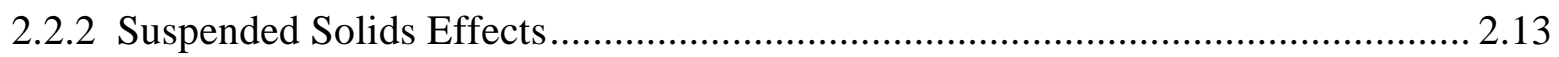

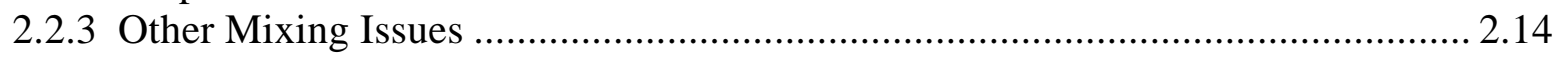

2.3 Reanalysis of Historical Gas Release Data …….................................................... 2.14

3.0 Analysis of Gas Releases Induced by Supernate Decant .................................................. 3.1

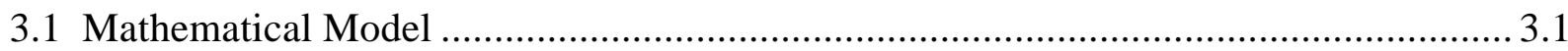

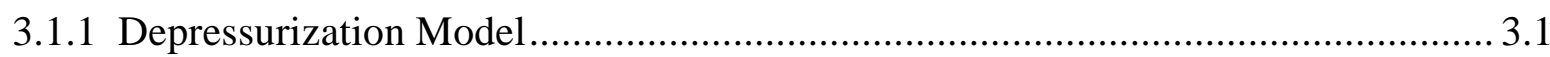

3.1.2 Buoyancy Threshold and Gas Release/Material Suspension Models ........................ 3.5

3.1.3 Headspace Hydrogen Concentration Model ........................................................... 3.10

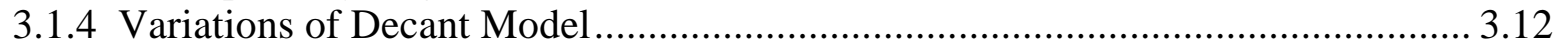

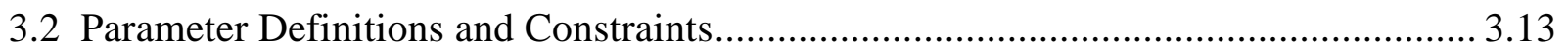

3.2.1 Overall Tank and Waste Configuration and Properties.......................................... 3.14

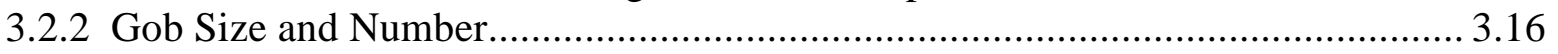

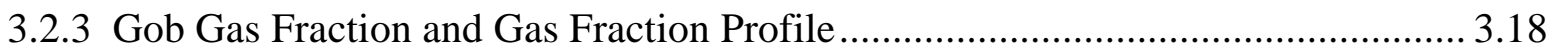

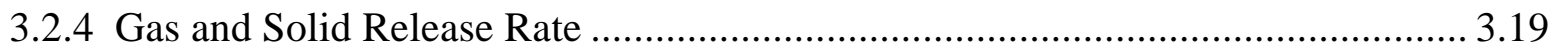

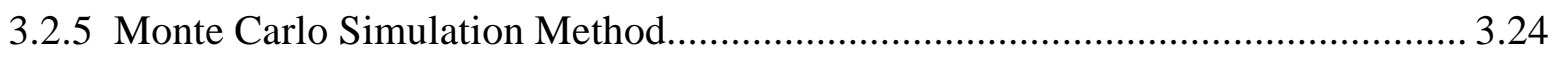

3.3 Analysis of GREs Induced by Supernate Decant in AN-105 .........................................2.25

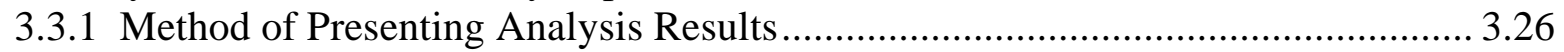

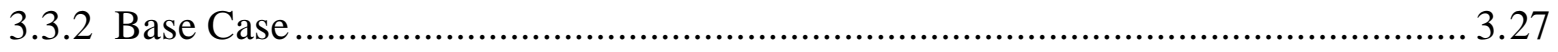

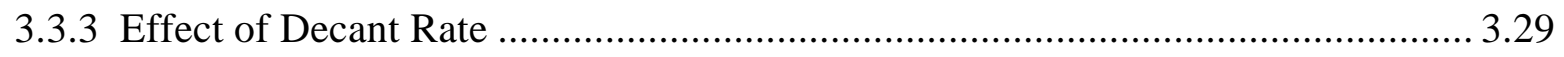

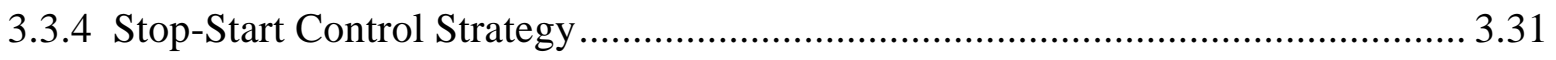

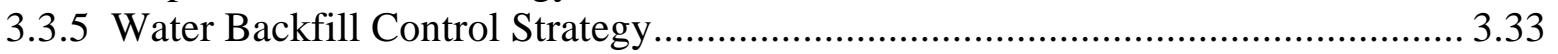

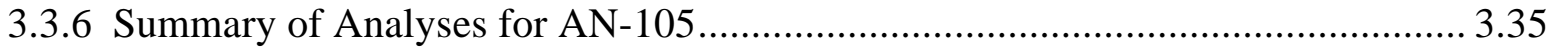

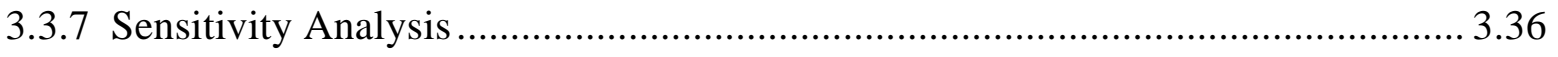




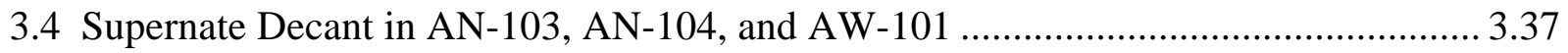

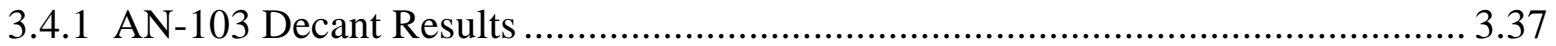

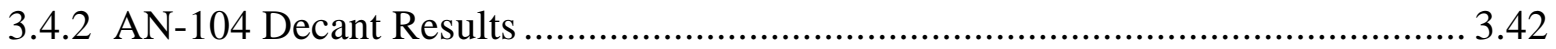

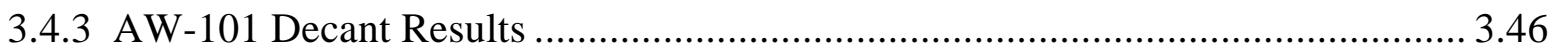

3.5 Conclusions from Supernate Decant Modeling Results............................................... 3.50

4.0 Analysis of Gas Releases Induced by Mixer Pump Operation.............................................. 4.1

4.1 Modeling the Effect of the Mixer Pump Jet on the Waste .............................................. 4.1

4.1.1 The Theory of Jet-Induced Gas Release ................................................................... 4.2

4.1.2 Observations and Data from Initial Mixing of SY-101 …………………….......... 4.7

4.1.3 Energy Balance Model for Waste Disturbance..................................................... 4.17

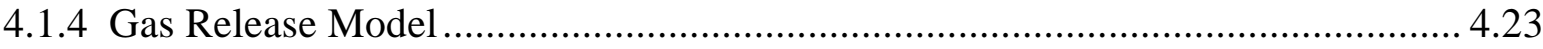

4.1.5 Recommendations for Further Modeling and Validation ..................................... 4.25

4.2 Model for Direct and Induced Gas Releases ............................................................. 4.27

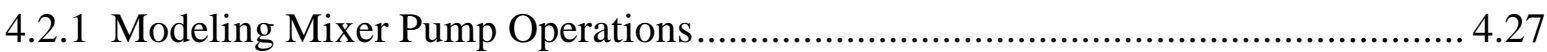

4.2.2 Partitioning the Waste Disturbance Among Gobs ............................................. 4.28

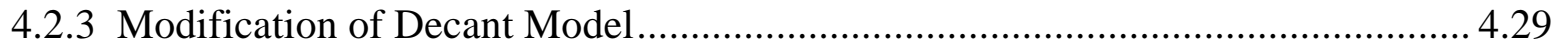

4.3 Parameter Definitions and Constraints........................................................................... 4.31

4.4 Analysis of Mixing-Induced Gas Releases in AN-105 ..................................................3. 4.3

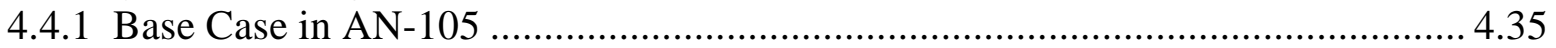

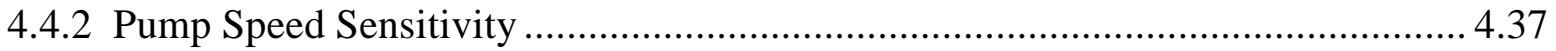

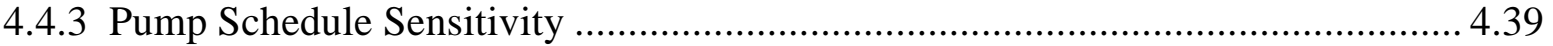

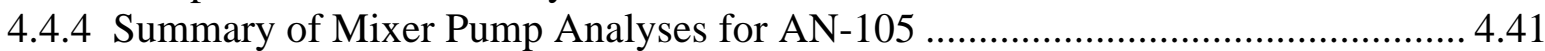

4.5 Analysis of Mixing-Induced Gas Releases in Other Tanks.........................................4.42

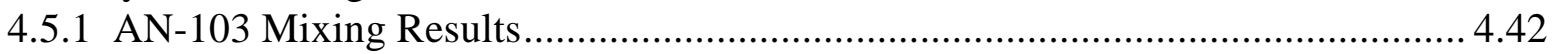

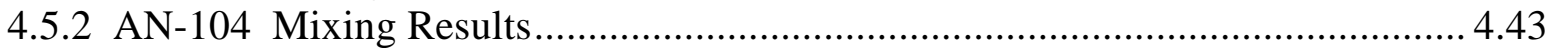

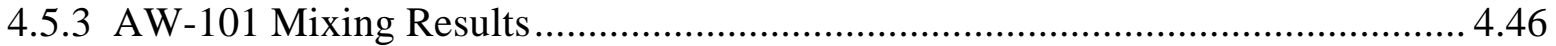

4.6 Conclusions from Mixing-Induced Gas Release Modeling Results .............................. 4.48

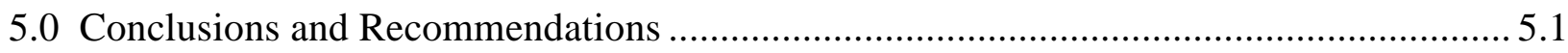

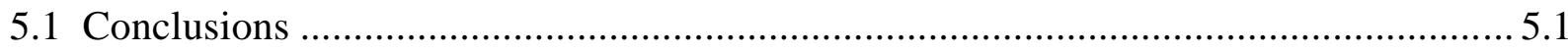

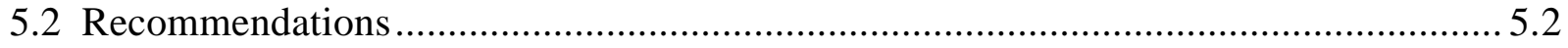

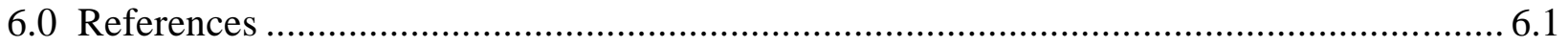

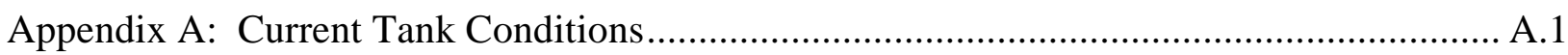

Appendix B: Determination of the Rise Distance of a Buoyant Gob.......................................... 1

Appendix C: Model Parameter Probability Distributions............................................................ 1

Appendix D: Electronic Input Distributions, Decant and Mixing Models, and Results 


\section{Figures}

1.1 Predicted Headspace Hydrogen Concentration in AN-105 During Supernate Decant $^{\circ}$ from $^{\circ}$ Previous Analysis........................................................................... 1.6

1.2 Hydrogen Concentration and Waste Level During SY-101 Phase B Mixing ................ 1.7

2.1 Change in Parabolic Void Profile from Initial Profile During Decant.......................... 2.5

2.2 Shift in Linear Void Profile from Initial Profile During Decant ................................ 2.5

2.3 GRE in AN-104 on 10/2/95, Comparing Calculated and Measured Headspace

Hydrogen ${ }^{\circ}$ Concentrations............................................................................. 2.16

2.4 GRE in AN-105 on 7/29/99, Comparing Calculated and Measured Headspace

Hydrogen ${ }^{\circ}$ Concentrations................................................................................... 2.17

2.5a Examples of GREs that Consist of Multiple Releases .............................................2.18

2.5b GRE in AN-104 on 6-2-00, Showing Model Fit to All Data Without

Differentiation of Separate ${ }^{\circ}$ Releases................................................................... 2.18

2.6 GRE in AN-104 on 6-2-00, Parsed into Multiple Releases....................................... 2.19

2.7 Large GREs that Appear to Consist of Multiple Releases .....................................2.21

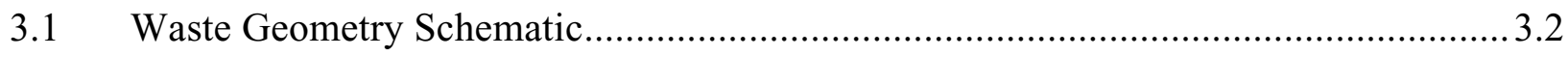

3.2 Linear and Parabolic Gas Fraction Profiles ............................................................ 3.4

3.3 Schematic of Tank Flow Paths ..................................................................... 3.11

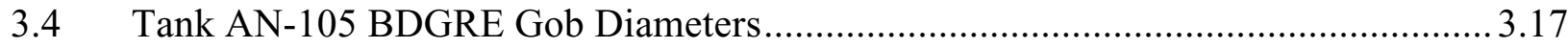

3.5 Tank AN-105 Time to Peak Release Rates for Spontaneous BDGREs.......................3.20

3.6 Number of Gobs as a Function of F: Model Results, AN-105 .............................. 3.21

3.7 Fraction of Gas Released as a Function of F: Model Results, AN-105 ................... 3.22

3.8 Fraction of Tank Area Participating as a Function of F: Model Results, AN-105 ....... 3.22

3.9 Time Between Gobs as a Function of F: Model Results, AN-105 ............................ 3.23

3.10 Time Between Gobs as a Function of the Time to Peak Release Rate ........................ 3.24

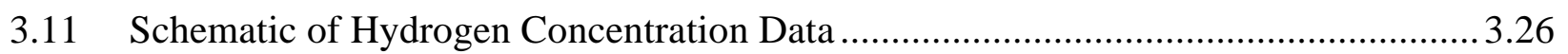

3.12 Derivation of Output Probability Distribution.................................................. 3.27

3.13 Peak Hydrogen Concentration for the AN-105 Base Case .......................................3.28

3.14 Gas Release Fraction for the AN-105 Base Case ................................................. 3.28

3.15 Representative Hydrogen Concentration History for the AN-105 Base Case...............3.29

3.16 Hydrogen Concentration for 30 gpm Decant Rate in AN-105 .................................3.30

3.17 Fraction of Gas Released for 30 gpm Decant Rate in AN-105 .................................3.30

3.18 Peak Hydrogen Concentration for the Stop-Start Case in AN-105 ........................... 3.31

3.19 Gas Release Fraction for the Stop-Start Case in AN-105 ......................................3.32

3.20 Hydrogen Concentration Transient for Stop-Start Case in AN-105 .......................... 3.32

3.21 Peak Hydrogen Concentration for Water Backfill in AN-105 ..................................3.34

3.22 Fraction of Gas Released for Water Backfill in AN-105 ........................................ 3.34

3.23 Hydrogen Concentration Transient for Water Backfill in AN-105 ........................... 3.35

3.24 Peak Hydrogen Concentration for the AN-103 Base Case .....................................3.38 
3.25 Gas Release Fraction for the AN-103 Base Case .....................................................3.38

3.26 Peak Hydrogen Concentration for 30 gpm Decant Rate in AN-103............................3.39

3.27 Gas Release Fraction for 30 gpm Decant Rate in AN-103 ........................................3.39

3.28 Peak Hydrogen Concentration for the Stop-Start Case in AN-103 ...............................3.40

3.29 Gas Release Fraction for the Stop-Start Case in AN-103 ………................................4.

3.30 Peak Hydrogen Concentration for Water Backfill in AN-103 ........................................

3.31 Gas Release Fraction for Water Backfill in AN-103 ..................................................41

3.32 Peak Hydrogen Concentration for the AN-104 Base Case ..........................................3.42

3.33 Gas Release Fraction for the AN-104 Base Case ........................................................43

3.34 Peak Hydrogen Concentration for 30 gpm Decant Rate in AN-104...............................43

3.35 Gas Release Fraction for 30 gpm Decant Rate in AN-104 ..........................................4

3.36 Peak Hydrogen Concentration for the Stop-Start Case in AN-104 ...............................4.

3.37 Gas Release Fraction for the Stop-Start Case in AN-104 ……....................................45

3.38 Peak Hydrogen Concentration for Water Backfill in AN-104 ……..............................4.45

3.39 Gas Release Fraction for Water Backfill in AN-104 ....................................................4.46

3.40 Peak Hydrogen Concentration for the AW-101 Base Case............................................4.

3.41 Gas Release Fraction for the AW-101 Base Case ………...........................................4.

3.42 Peak Hydrogen Concentration for 30 gpm Decant Rate in AW-101 .............................4.

3.43 Gas Release Fraction for 30 gpm Decant Rate in AW-101 ..........................................48

3.44 Peak Hydrogen Concentration for Water Backfill in AW-101 ....................................3.49

3.45 Gas Release Fraction for Water Backfill in AW-101 ……………..............................3.49

4.1 Gas Release from SY-101 Mixer-Pump Correlated with Jet Momentum Scaling 4.9

4.2 Gas Release from SY-101 Mixer Pump Correlated with Jet Volume Flow Scaling ..... 4.10

4.3 Gas Release from SY-101 Mixer Pump Correlated with Jet Hydraulic Power Scaling 4.10

4.4 July 26, 1993 Pump Run and GC Data ………….................................................... 4.11

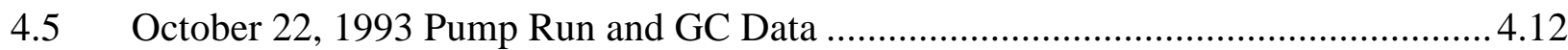

4.6 November 5, 1993 Pump Run and GC Data ..............................................................12

4.7 November 10, 1993 Pump Run and GC Data.............................................................. 43

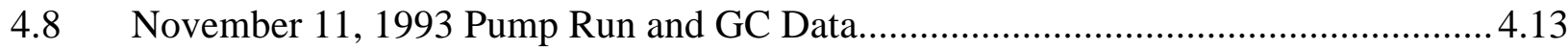

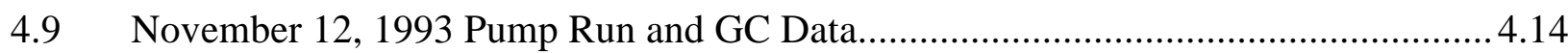

4.10 November 13, 1993 Pump Run and GC Data............................................................

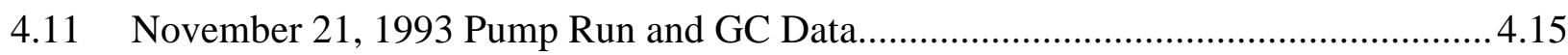

4.12 Relation Between Peak $\mathrm{H}_{2}$ Delay and Total Gas Release ............................................ 4.15

4.13 Relation Between Peak $\mathrm{H}_{2}$ Delay and Jet Flow Rate Scaling ..................................... 4.16

4.14 Relation Between Peak $\mathrm{H}_{2}$ Delay and Jet Hydraulic Power Scaling ............................4.16

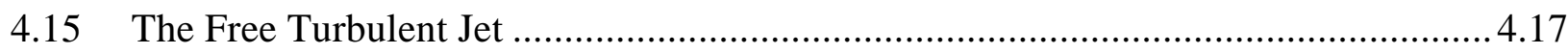

4.16 A Model for Jet Penetration into a Pseudo Solid ....................................................... 4.19

4.17 Gas Release Model Results .................................................................................2

4.18 Comparison of Predicted Gas Release from Model and Observed Release in SY-101.4.24

4.19 Predicted Gas Release Volume from Operation of a Mixer Pump in Tank AN-105..... 4.25 
4.20 Schematic of Mixer Pump Operation and Effect on Gob Distribution...........................4.29

4.21 Distribution for Time to Peak Gas Release Rate for Mixer Pump Operations............... 4.32

4.22 Distribution for Fraction of Solids, Mobilized by Pump, that Remain Suspended ....... 4.33

4.23 Peak Hydrogen Concentration: 5\% Disturbance Every 24 Hours in AN-105............... 4.35

4.24 Gas Released by BDGREs: 5\% Disturbance Every 24 Hours in AN-105 ………......... 4.36

4.25 Example Simulation Hydrogen Concentration: 5\% Disturbance

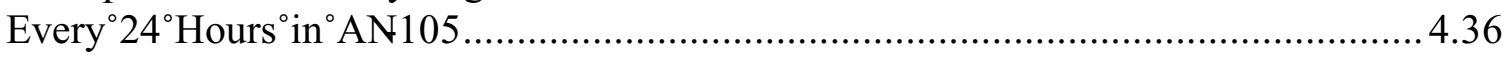

4.26 Peak Hydrogen Concentration: 20\% Disturbance Every 24 Hours in AN-105............. 4.37

4.27 Gas Released by BDGREs: 20\% Disturbance Every 24 Hours in AN-105 .................. 4.38

4.28 Example Simulation Hydrogen Concentration in AN-105 ……................................38

4.29 Peak Hydrogen Concentration: 5\% Disturbance Every 8 Hours in AN-105 ................. 4.39

4.30 Gas Released by BDGREs: 5\% Disturbance Every 8 Hours in AN-105 ……............... 4.40

4.31 Example Simulation Hydrogen Concentration: 5\% Disturbance

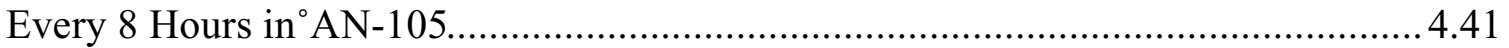

4.32 Peak Hydrogen Concentration: 5\% Disturbance Every 24 Hours in AN-103 .............. 4.42

4.33 Gas Released by BDGREs: 5\% Disturbance Every 24 Hours in AN-103 .................... 4.43

4.34 Peak Hydrogen Concentration: 5\% Disturbance Every 24 Hours in AN-104 .............. 4.44

4.35 Gas Released by BDGREs: 5\% Disturbance Every 24 Hours in AN-104 ……..............4.44

4.36 Peak Hydrogen Concentration: 20\% Disturbance Every 24 Hours in AN-104.............. 4.45

4.37 Gas Released by BDGREs: 20\% Disturbance Every 24 Hours in AN-104 ….............. 4.45

4.38 Peak Hydrogen Concentration: 5\% Disturbance Every 24 Hours in AW-101...............4.46

4.39 Gas Released by BDGREs: 5\% Disturbance Every 24 Hours in AW-101 ................... 4.47

4.40 Peak Hydrogen Concentration: 20\% Disturbance Every 8 Hours in AW-101 ............... 4.47

4.41 Gas Released by BDGREs: 20\% Disturbance Every 8 Hours in AW-101 ................... 4.48

\section{Tables}

1.1 Design Parameters of the SY-101 and W-211 Mixing Pumps ..................................... 1.8

2.1 Comparing Single- and Multiple-Release Analysis Results for AN-104 ....................2.20

2.2 Comparing Single- and Multiple-Release Analysis Results for Different GREs..........2.2.22

2.3 Summary of Gas Release Calculations for AN-103, AN-104, AN-105, AW-101 _.......2.23

3.1 Select Hanford DST Waste Properties and Energy Ratio Results .................................... 3.7

3.2 Select BD Experiments Material Properties and Energy Ratio Results............................3.7

3.3 Tank and Waste Configuration Parameters ................................................................. 3.14

3.4 Tank and Waste Configuration Parameters: Values and Distributions........................... 3.15

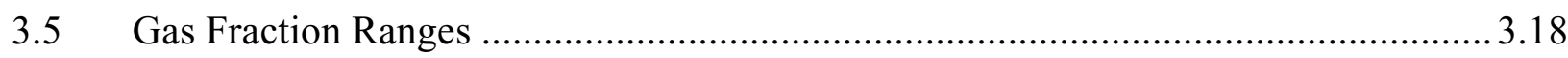

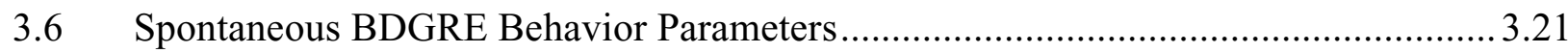

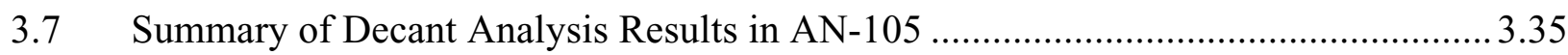

3.8 Sensitivity Results from Base Case ....................................................................3.36 
3.9 Summary of Decant Analysis Results ..................................................... 3.50

4.1 SY-101 Pump Runs that Disturbed Fresh Waste.................................................... 4.8

4.2 Gas Releases Associated with SY-101 Pump Runs of Interest ................................4.9

4.3 Model Input Parameters for Tank SY-101 Mixer Pump Tests .................................. 4.23

$4.4 \quad$ Model Parameter Values for Tank AN-105. .................................................... 4.25

4.5 Fraction of Sediment Disturbed for Given Mixer Pump Run Conditions.................... 4.34

4.6 Summary of Mixer Pump Analysis Results in AN-105 ...................................... 4.41

4.7 Summary of Mixing Analysis Results ............................................................. 4.49 


\subsection{Introduction}

This report describes an investigation performed by Pacific Northwest National Laboratory (PNNL) for CH2M HILL Hanford Group, Inc. (CHG) on potential flammable gas releases from proposed retrieval operations in four double-shell tanks (DSTs). The tanks are 241-AN-103, -AN-104, -AN-105, and -AW-101. The operations, which involve removing or decanting the supernatant liquid or mixing the waste with two jet mixer pumps, or both, are planned for waste feed delivery to the vitrification plant in 2009-2019. Such operations are challenging in these tanks because each of them has exhibited spontaneous buoyant displacement gas release events (BDGREs) that have ranged from less than 1 to more than 30 standard cubic meters of gas (30-1,200 scf) containing 30 to $60 \%$ hydrogen. Only twice have these releases raised the headspace hydrogen concentration to more than $25 \%$ of the lower flammability limit (LFL) in AN-105 during the six to seven years that headspace hydrogen concentration has been monitored. $^{\text {(a) }}$ However, all four tanks retain enough gas to reach or exceed the LFL if a large enough fraction of the retained gas volume were released suddenly (Hedengren et al. 2000).

Given this relatively large volume of retained gas, retrieval operations could potentially create a hazardous gas release. The large mixer pumps that are planned to be installed have the capability of mobilizing most of the waste in the tank. Upon pump startup, they could release a large fraction of a tank's stored gas in a short time. Decanting the supernatant liquid can trigger a series of BDGREs by reducing the hydrostatic pressure and causing the stored gas to expand. Both operations therefore need to be planned carefully to ensure they can be performed safely.

This report presents the results of a detailed model of the gas release behavior during both supernate decant and initial mixer pump operation that puts the potential for induced gas releases in perspective. Section 2 describes the search for mechanisms that could exacerbate gas release during decant or mixing; Section 3 analyzes gas releases during supernate decant, including the mathematical model, parameter definitions, and analysis results. Section 4 analyzes gas releases during initial mixer pump operation, and Section 5 presents the conclusions and recommendations from the study. Section 6 lists the cited references. Appendixes are provided with detailed tank waste configuration waste properties and an analysis of observed gas release behavior. Appendix D is a CD containing the input data sets, models, and outputs of the Monte Carlo simulations, and spreadsheets with analyses of historic gas releases.

The rest of Section 1 provides important contextual information upon which the main body of the report is built. Section 1.1 contains background information on flammable gas release behavior, Section 1.2 summarizes the current understanding of the effects of supernate decanting and some earlier calculation results, and Section 1.3 describes initial mixer pump operation in SY-101 and an earlier degassing plan developed for AN-105.

(a) The peak headspace hydrogen concentration in AN-105 was $1.45 \%$ on $5 / 30 / 96,1.75 \%$ on $8 / 21 / 96$, and $0.689 \%$ on $4 / 5 / 97$. One-fourth of the LFL in AN-105 is $0.98 \%$ hydrogen; the administrative action level for monitoring is $0.625 \%$ hydrogen. The LFL for hydrogen is approximately $4.00 \%$ (40,000 ppm). 


\subsection{Background}

There are 149 are single-shell tanks (SSTs) with capacities from 50 to 1,000 kgal. These tanks were built in the 1940s, 1950s, and early 1960s. In the 1970s and 1980s, 28 DSTs of $1,200 \mathrm{kgal}$ capacity were constructed. Because of concerns about potential and actual leaks, the SSTs were removed from active use in 1980, and much of their liquid waste has been transferred to DSTs. To conserve tank space, the liquid in the DSTs was typically concentrated by evaporation, which, in some tanks, formed relatively deep layers of sediment as sodium salts precipitated from the cooling liquid.

All Hanford radioactive wastes generate flammable gases by radiolysis of water, decomposition of organic compounds, and corrosion of the steel tank walls. The main fuel components of the waste gas mixture are hydrogen and, at much lower concentrations, ammonia. The gas also contains nitrous oxide (an oxidizer at temperatures above 1,000K), nitrogen (inert component), and very small amounts of methane and other hydrocarbons. The gas mixture can be ignited if its concentration exceeds the LFL. Hydrogen, with an LFL of 4 vol\% in air, typically dominates the flammability of the waste gas (Mahoney et al. 2000). Accounting for the effects of the other fuel gases reduces the LFL slightly to $3.7-3.9 \mathrm{vol} \%$.

Flammable gas generation by itself is not a hazard if the gas is released continuously as fast as it is generated. The rate of gas generation is much slower than the headspace ventilation rate, and the mixing is rapid. The highest gas generation rates are less than 100 scf per day, while the nominal DST ventilation rate is 100 scf per minute. Consequently, the background concentration of flammable gas in the headspace of each of the DSTs has remained far below the LFL.

In some DST wastes, specifically those with relatively deep layers of supernate and sediment, the generated gas can accumulate in the sediment until a relatively large volume is released rapidly into the tank headspace. This is a BDGRE. Such sudden releases potentially could cause the concentration of the flammable gas mixture in the headspace to reach the LFL, potentially leading to deflagration if an ignition source were present while the tank headspace remained flammable. If the energy in the deflagration were sufficient, the tank could be damaged and radioactive waste released.

The four tanks covered in this report, AN-103, AN-104, AN-105 and AW-101, as well as SY-103, all exhibit BDGREs, though none have made the tank headspace flammable since gas monitoring equipment was installed in 1994-95. A detailed analysis of recorded gas releases of these tanks is given in Section 2.3, and measurements of the waste temperature, level, and GRE history are provided in Appendix A. Tank SY-101 had the largest BDGREs, three of which caused the headspace to exceed the LFL before mixer pump operation halted them in 1993. The intense study of the behavior of this tank led to a general understanding of BDGREs and how to quantify and predict them to some degree.

The data and observations pertaining to BDGREs indicate that the sediment layer consists of a collection of about 10 to 15 regions, or "gobs," in different stages of gas retention (Meyer et al. 1997; Meyer and Stewart 2001). A BDGRE occurs when one or more of these gobs of sediment layer accumulates enough gas to become buoyant with respect to the liquid above it. The 
buoyant gob rises, and the gas it contains expands as the hydrostatic pressure decreases. The expanding gas bubbles yield the surrounding waste and escape into the headspace as the gob disintegrates at the waste surface. The onset of buoyancy and the subsequent gas release are summarized here and treated in more detail in Section 3.

For the gob to initiate a BDGRE, it must be slightly more than buoyant to overcome the restraining force of the sediment strength. That is, $\alpha_{\mathrm{gob}}=\alpha_{\mathrm{C}}$, the critical gas fraction, defined by Meyer et al. (1997) as

$$
\alpha_{C}=\alpha_{N B}+\frac{\beta \tau_{y}}{\rho_{S} g h_{S}}
$$

where $\tau_{\mathrm{y}}$ is the yield stress in shear, $\rho_{\mathrm{S}}$ is the bulk degassed sediment density, $\mathrm{g}$ is the acceleration of gravity, and $h_{s}$ is the sediment depth. The coefficient, $\beta$, is the ratio of the yield stress in tension to the yield stress in pure shear. The stress at yielding may be given by $\beta \tau_{\mathrm{y}}$, where $1 \leq \beta \leq \sqrt{3}$. In this study, $\beta=1$ is used because this provides good correlation between the measured and calculated gas fractions in select DSTs (Meyer et al. 1997). The neutral buoyancy void fraction, $\alpha_{\mathrm{NB}}$, is defined in terms of the densities of the liquid and sediment as

$$
\alpha_{\mathrm{NB}}=1-\frac{\rho_{\mathrm{L}}}{\rho_{\mathrm{S}}}
$$

where $\rho_{\mathrm{L}}$ is the supernatant liquid density. Eq. (1.2) shows that increasing the liquid density lowers the buoyancy point.

The volume of gas released in a BDGRE is determined from the specific gob geometry and void fraction. The in situ volume of gas in a right circular cylindrical gob is given by

$$
\mathrm{V}_{\text {gas }}=\mathrm{h}_{\mathrm{S}} \frac{\pi}{4} \mathrm{D}_{\text {gob }}^{2} \alpha_{\text {gob }}
$$

where $\mathrm{D}_{\mathrm{gob}}$ is the gob diameter and $\alpha_{\mathrm{gob}}$ is its gas volume fraction. At the moment the gob breaks free and begins to rise to the surface, $\alpha_{\text {gob }}=\alpha_{C}$. After the gob has risen to the surface, the volume of gas that is released to the tank headspace is described by

$$
\mathrm{V}_{\text {rel }}=\frac{\mathrm{P}_{\mathrm{S}}}{\mathrm{P}_{\mathrm{A}}} \mathrm{f}_{\text {rel }} \mathrm{V}_{\text {gas }}
$$

where $f_{\text {rel }}$ is the fraction of gas released from a gob assuming return to neutral buoyancy at the surface. The average pressure of the gas in the sediment layer, $\mathrm{P}_{\mathrm{S}}$, is given by

$$
\mathrm{P}_{\mathrm{S}}=\mathrm{P}_{\mathrm{A}}+\rho_{\mathrm{L}} \mathrm{g}\left(\mathrm{h}_{\mathrm{C}}+\mathrm{h}_{\mathrm{L}}+\frac{\mathrm{h}_{\mathrm{S}}}{2}\right)
$$


where $P_{A}$ is the atmospheric pressure, $h_{C}$ is the crust layer thickness, and $h_{L}$ is the supernate depth. The release fraction, $\mathrm{f}_{\text {rel }}$, is given by

$$
\mathrm{f}_{\text {rel }}=1-\frac{\alpha_{\mathrm{NB}}\left(1-\alpha_{\mathrm{C}}\right)}{\alpha_{\mathrm{C}}\left(1-\alpha_{\mathrm{NB}}\right)} \frac{\mathrm{P}_{\mathrm{A}}}{\mathrm{P}_{\mathrm{S}}}
$$

After the gob has released the portion of its gas defined by Eq. (1.6) and compresses as it falls back to the sediment layer, its gas volume fraction will be reduced to

$$
\alpha_{\mathrm{gob}}=\left[\frac{\mathrm{P}_{\mathrm{S}}}{\mathrm{P}_{\mathrm{A}}} \frac{\left(1-\alpha_{\mathrm{NB}}\right)}{\alpha_{\mathrm{NB}}}+1\right]^{-1}
$$

While a buoyant displacement can occur when the gas volume fraction in the sediment reaches the critical value, gas release will be minimal unless the buoyant potential energy exceeds the energy required to yield the displaced material. A large fraction of the buoyant energy is dissipated in other processes, so the required buoyant energy is much greater than that which would just yield the waste. Stewart et al. (1996) developed an energy model to account for this. The ratio between the buoyant energy, $E_{b}$, and the energy required to yield the gasbearing gob participating in the buoyant displacement, $\mathrm{E}_{\mathrm{y}}$, is termed the "energy ratio" and may be expressed as

$$
\frac{E_{b}}{E_{y}}=\frac{\alpha_{C} \rho_{L} g h}{\left(1-\alpha_{C}\right) \varepsilon_{y} \tau_{y}}\left[\left(1+\frac{1}{\gamma}\right) \ln (1+\gamma)-k\right]
$$

where $\mathrm{h}$ is the distance from the center of the participating gob to the top of the liquid layer, and $\varepsilon_{\mathrm{y}}$ is strain at failure, taken to be unity. The parameters $\gamma$ and $\mathrm{k}$ are

$$
\begin{aligned}
& \gamma=\frac{\rho_{\mathrm{L}} \mathrm{gh}}{\mathrm{P}_{\mathrm{A}}} \\
& \mathrm{k}=\frac{\alpha_{\mathrm{NB}}\left(1-\alpha_{\mathrm{C}}\right)}{\alpha_{\mathrm{C}}\left(1-\alpha_{\mathrm{NB}}\right)}
\end{aligned}
$$

Based on experimental observations and tank behavior, gas can be released when the energy ratio exceeds 3, and significant releases [approximating the release fraction defined by Eq. (1.6)] can occur above energy ratios of 5 . $^{\text {(a) }}$

Because AN-103, AN-104, AN-105, and AW-101 exhibit spontaneous BDGREs at a nearly random frequency, it is impossible to compute a "window" for intrusive activities during which

(a) A more precise definition of rise distance (h) that accounts for the actual buoyancy of the gob at the surface is developed in Section 3. The energy ratio thresholds for gas release with this definition are adjusted accordingly. 
no gas releases will happen. Releases of as much as 10 to 20 cubic meters of gas can be expected and should be planned for during any operation in these tanks. At the same time, the global waste disturbances resulting from supernate decanting and initial mixing can induce additional BDGREs. Section 1.2 describes how this occurs during supernate decant.

\subsection{Supernate Decanting}

The supernate is planned to be decanted by a transfer pump installed just above the top of the sediment layer. As the liquid is removed at constant rate, the hydrostatic pressure on the gas in the sediment layer decreases and causes it to expand increasing the gas volume fraction. This is the mechanism that triggers gas release during decant. Gobs with a sufficient initial gas volume fraction will become buoyant and undergo BDGREs.

Once buoyant, rising gobs are assumed to behave like those participating in spontaneous gas releases. The initial gob size and gas fraction distributions are determined by tank historical behavior and measured data, as is the actual gas release behavior when a BDGRE occurs during decant. However, as the supernate depth decreases the tank headspace increases, so the same gas release will produce a progressively lower hydrogen concentration. At some point, the supernate depth will be insufficient to provide the energy required to yield the waste, as defined by Eq. (1.8), and gas releases will effectively cease. Thus the highest hydrogen concentrations resulting from induced BDGREs can be expected early in the process.

The possibility of these potential BD GREs to occur and to reach the LFL was investigated for Tank AN-105 in 1999. (a) This analysis assumed an initial gob size distribution dictated by the sizes of circles, representing individual gobs, that can be fit inside a larger circle representing the tank. The distribution was chosen such that the mean gob diameter was consistent with the mean historic gas release volumes (from Meyer et al. 1997). Initial gas volume fractions equally spaced from neutral buoyancy (Eq. 1.1) down to the minimum value (approximately as given by Eq. 1.7) were assigned to each gob. The largest gob was conservatively assigned the largest gas fraction. This resulted in the modeled tank having about twice the retained gas volume as actually measured. The decant rate was set at $100 \mathrm{gpm}$.

Figure 1.1 shows the predicted headspace hydrogen concentration over the entire decant process. Because of the linear distribution of gas fraction over the gobs, the induced BDGREs are evenly spaced. Despite the conservative gas volume, the peak headspace hydrogen concentration was well below the LFL, though it exceeded the action level of 6,250 ppm (CHG 2000) during the first two BDGREs. The peak hydrogen concentration for the largest induced release was roughly equivalent to the largest historical GRE, where the hydrogen concentration reached $17,000 \mathrm{ppm}$. Only five of the ten gobs contained enough gas initially to become buoyant during the decant. These results are consistent with those of the much more complete and detailed analysis presented in Section 3.

(a) PNNL letter report TWS99.44 Rev. 1, August 1999. Potential for Inducing Gas Releases in DoubleShell Tanks During Retrieval, by CW Stewart, PA Meyer, and BE Wells. 


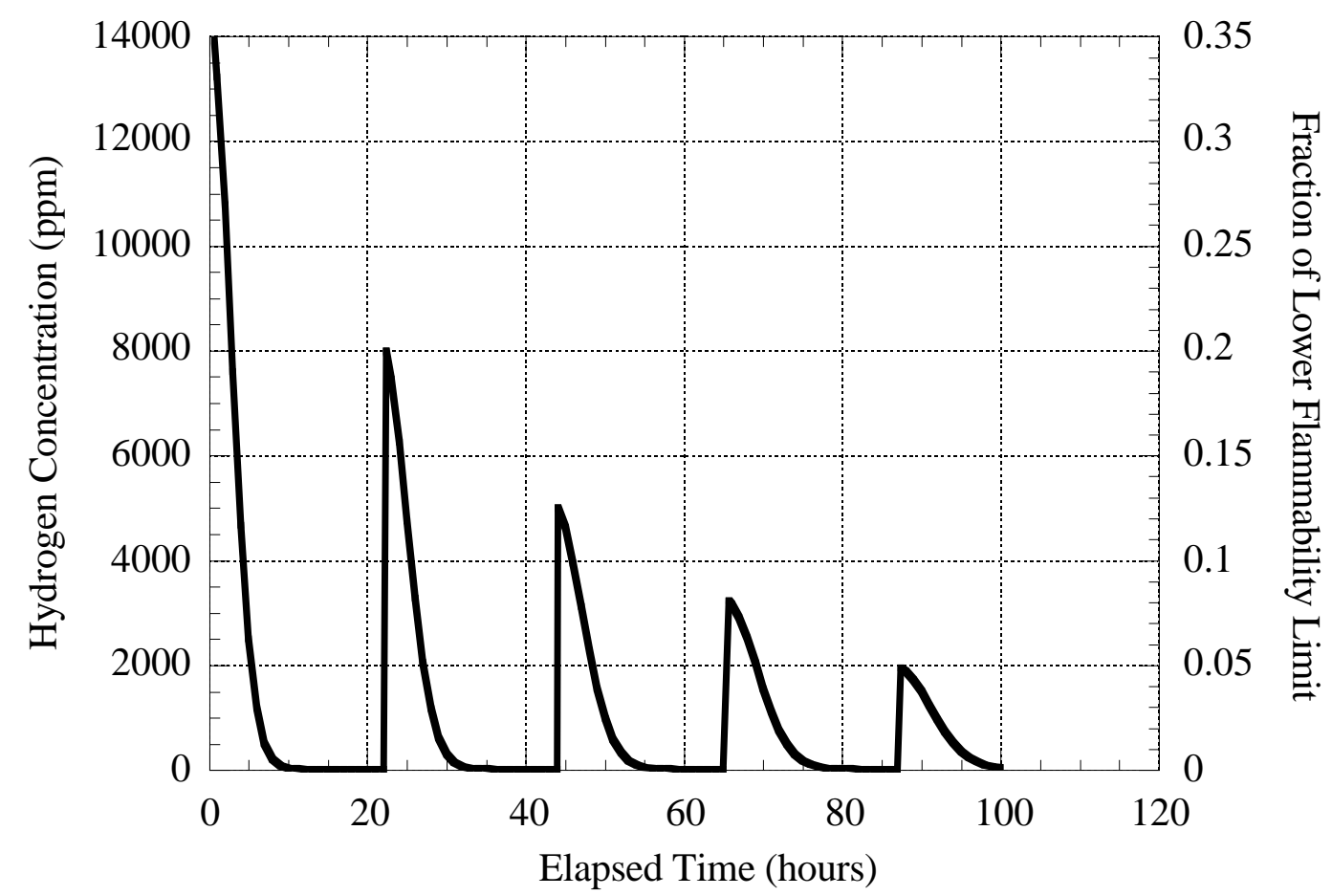

Figure 1.1. Predicted Headspace Hydrogen Concentration in Tank AN-105 During Supernate Decant from Previous Analysis ${ }^{(a)}$

\subsection{Mixer Pump Degassing}

Jet mixing is a mechanical disturbance that has the potential to affect a large waste volume at a relatively high rate. Mixer pump operation in SY-101 provided insight into gas releases induced by mechanical disruption (Allemann et al. 1994; Brewster et al. 1995). The pump was installed on July 3, 1993, just over a week after a 6,000-scf spontaneous BDGRE. Initial pump operations (Phase A) were extremely gentle because of concerns that pump operation might trigger a major GRE. This initial, low-speed testing did very little to disturb the waste or release gas, but it did show that brief 5-minute pump runs called "bumps" were required to prevent nozzle plugging. Bumps continued about every other day up to the start of high-speed testing on October 21, 1993. One significant induced BDGRE and two much smaller releases were observed during this period, indicating that some regions of the tank were likely near buoyant.

High-speed mixer pump testing (Phase B) began on October 21, 1993 and continued until December 17, 1993. Testing consisted of a series of pump runs aimed into undisturbed waste and lasting from 20 to 30 minutes at up to almost full speed of $920 \mathrm{rpm}$. Figure 1.2 shows the daily peak hydrogen concentrations and the waste surface level during Phase B testing (Allemann et al. 1994). A large volume of gas was released during the first few weeks.

(a) PNNL letter report TWS99.44 Rev. 1, August 1999. Potential for Inducing Gas Releases in DoubleShell Tanks During Retrieval, by CW Stewart, PA Meyer, and BE Wells. 


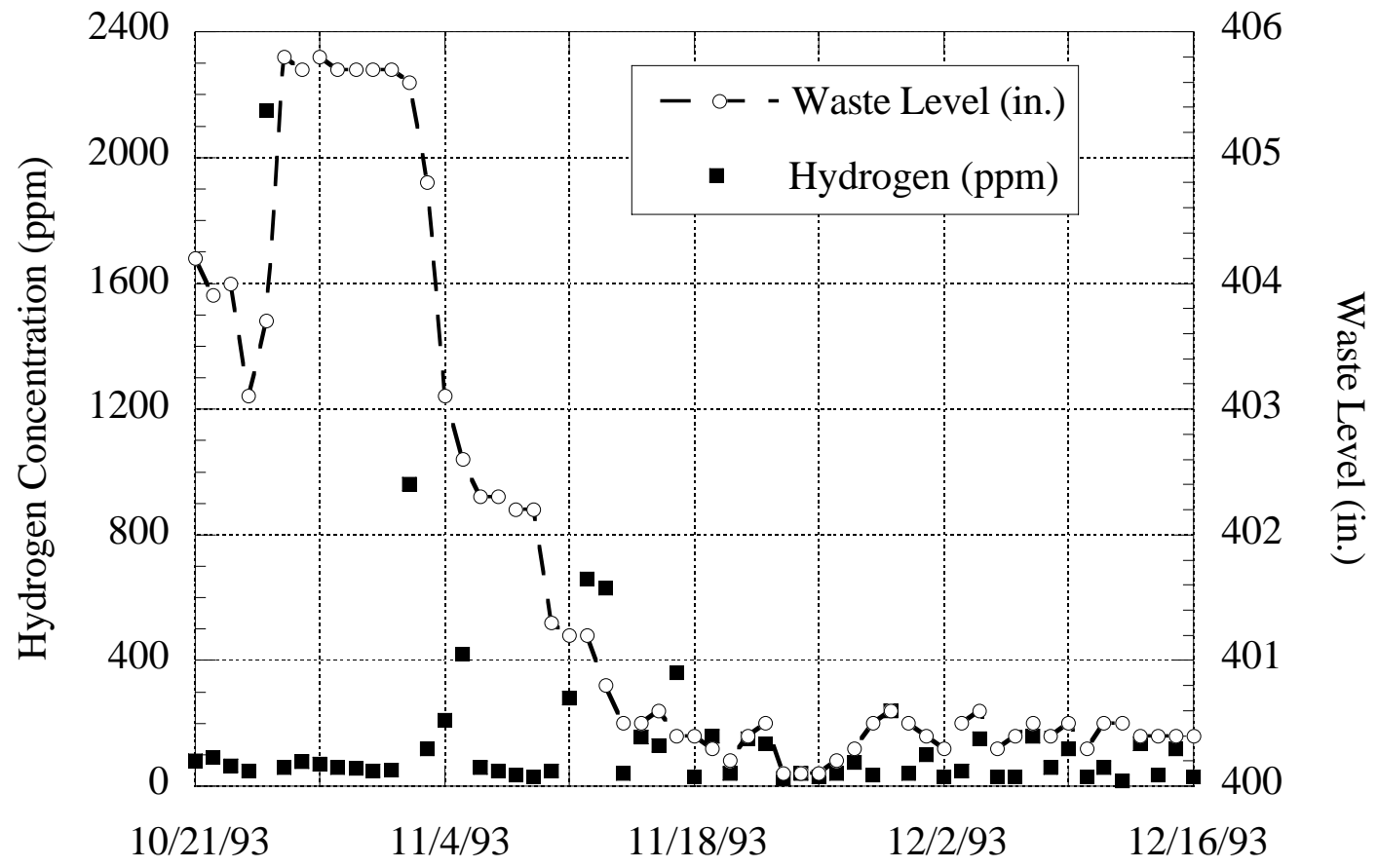

Figure 1.2. Hydrogen Concentration and Waste Level During SY-101 Phase B Mixing

The sudden level rise and high hydrogen concentration on November 1 is a clear example of an induced BDGRE, although the gas release was delayed several days. Two runs, at 320 and $520 \mathrm{rpm}$, had been made with the jets turned 30 degrees into undisturbed waste. The event occurred during the third run in the same direction at $920 \mathrm{rpm}$. A significant gas release accompanied the level rise, and another somewhat smaller one signaled the later level drop. But because the level remained relatively constant for over a week before suddenly dropping again, the waste apparently floated on the surface without releasing its gas during that time. No further episodes of this kind were seen. By early December the jets had mixed most of the gas-bearing sediment layer because gas releases for the remainder of Phase B testing were relatively small.

A degassing strategy using the two mixer pumps proposed for retrieval of $\mathrm{AN}-105$ was developed based on the mitigation of SY-101 during Phase B pump operations (Caley et al. 1998). The mixer pumps proposed for AN-105 are significantly more powerful than the mixer pump in SY-101; at their minimum operating speed of $700 \mathrm{rpm}$, they approximate the maximum performance of the SY-101 mixer pump at its maximum practical speed of $1000 \mathrm{rpm}$. The degassing plan for AN-105 accommodated mixer pump differences by operating one pump at a time and limiting the duration. The differences in the two pumps are summarized in Table 1.1.

The maximum cumulative gas release for a single day in SY-101 during the Phase B testing was $14.3 \mathrm{~m}^{3}$ (well below that required to reach the LFL). This gas release resulted from four 20minute mixer pump runs at 360,510,720, and $920 \mathrm{rpm}$ at a fixed angle. The calculated energy supplied to the waste by the pump during this period was approximately $18.2 \mathrm{~kW}-\mathrm{hr}$ (Caley et al. 1998). A single mixer pump in AN-105, operated at $700 \mathrm{rpm}$ for 24 minutes, was calculated to 
Table 1.1. Design Parameters of the SY-101 and W-211 Mixing Pumps

\begin{tabular}{|c|c|c|}
\hline Parameters & SY-101 & AN-105 (W-211 Project) \\
\hline Power & $112 \mathrm{~kW}(150 \mathrm{hp})$ & $224 \mathrm{~kW}(300 \mathrm{hp})$ \\
\hline Speed & 100 to $1,180 \mathrm{rpm}$ & 700 to $1,200 \mathrm{rpm}$ \\
\hline Pumping capacity & $0.18 \mathrm{~m}^{3} / \mathrm{s}(2,800 \mathrm{gpm})$ & $0.69 \mathrm{~m}^{3} / \mathrm{s}(11,000 \mathrm{gpm})$ \\
\hline Inlet nozzle location & $\begin{array}{l}6.6 \mathrm{~m}(260 \mathrm{in} .) \text { above tank } \\
\text { bottom }\end{array}$ & $\begin{array}{l}17.8 \mathrm{~cm}(7 \text { in.) above tank } \\
\text { bottom }\end{array}$ \\
\hline $\begin{array}{l}\text { Discharge nozzle } \\
\text { location }\end{array}$ & $\begin{array}{l}71 \mathrm{~cm}(28 \text { in.) above tank } \\
\text { bottom }\end{array}$ & $\begin{array}{l}45.7 \mathrm{~cm}(18 \text { in.) above tank } \\
\text { bottom }\end{array}$ \\
\hline $\begin{array}{l}\text { Discharge nozzle } \\
\text { diameter }\end{array}$ & $6.6 \mathrm{~cm}(2.6$ in. $)$ & $15.2 \mathrm{~cm}(6$ in. $)$ \\
\hline Tank position & $\begin{array}{l}0.9 \mathrm{~m}(3 \mathrm{ft}) \text { west of tank } \\
\text { center }\end{array}$ & $\begin{array}{l}6.1 \mathrm{~m}(20 \mathrm{ft}) \text { north and } \\
\text { southeast of tank center } \\
\text { ( } 2 \text { pumps total) }\end{array}$ \\
\hline
\end{tabular}

supply equivalent energy for a roughly equivalent waste disturbance. It was suggested that during degassing each W-211 mixer pump be run individually at $700 \mathrm{rpm}$ for 24 minutes in varying directions to remove the bulk of the stored gas before being operated simultaneously at full power.

\subsection{Improving the Models and Predictions}

The work described above was performed in 1998 (Caley et al. 1998) and 1999. (a) During this time the most urgent safety issue at Hanford was the waste level growth phenomenon occurring in SY-101. The supernate decant and mixer pump degassing issues were relegated to low priority. Except for a scoping calculation for supernate decant in SY-103, ${ }^{(b)}$ no further analysis or development was performed.

In February and March of 2001, the Defense Nuclear Facility Safety Board (DNFSB) staff reviewed the earlier decanting model and results and questioned the assumption that induced GREs should be similar to historical spontaneous releases. In response to the DNFSB review, a workshop was held at PNNL in July 2001 to identify plausible mechanisms that could increase BDGREs beyond historical observations. Experts with considerable experience in the Hanford flammable gas issue participated from PNNL, CHG, and other contractors. The team developed a relatively long list of potential mechanisms that might exacerbate induced releases during both supernate decant and initial mixing.

(a) PNNL letter report TWS99.44 Rev. 1, August 1999. Potential for Inducing Gas Releases in DoubleShell Tanks During Retrieval, by CW Stewart, PA Meyer, and BE Wells.

(b) PNNL letter report TWS01.01, September 2000. A Strategy for Remediation and Return-to-Service for Tank 241-SY-103, by BE Wells and CW Stewart. 
The list of mechanisms was reviewed in detail, and calculations were performed as appropriate to evaluate whether they could have a significant effect. Several important mechanisms were selected for inclusion in improved models for the processes. The results were reviewed by an ad hoc committee of PNNL and CHG staff that included scientists, managers, and nuclear safety and licensing experts. The detailed explanation and disposition of each of the proposed mechanisms is the subject of Section 2.

The assimilation of the model improvements, development of input parameter distributions for the Monte Carlo analysis (itself a significant improvement in modeling strategy), and results of the improved models were reviewed during a series of meetings from August through November 2001. The results of the improved supernate decant model were presented to the DNFSB on November 19, 2001. The models and results are discussed in Sections 3 and 4. 


\subsection{Phenomena That Could Amplify Gas Releases}

This section describes potential mechanisms that could produce gas releases during supernate decanting and mixer pump degassing that are larger than those observed historically. The list was developed by a group of experts from PNNL and CHG in a workshop held July 3, 2001. Each of the proposed mechanisms was evaluated for inclusion in the enhanced gas release model described in Section 3. Those included are so indicated in the discussion. Phenomena active during supernate decanting are evaluated in Section 2.1 and those accompanying initial mixer pump operation in Section 2.2. A reanalysis of the historical gas release data is given in Section 2.3.

\subsection{Phenomena Active During Supernate Decanting}

Some phenomena relating to gas release may actually be caused by the decant process and do not occur during spontaneous GREs. These generally involve void growth during depressurization, which is shown to be different from accumulation of internally generated gas. These factors are generally included in the enhanced model for the depressurization process.

Other mechanisms take effect after a gob has become buoyant. Though the process of void growth to buoyancy is much faster during decant than during gas accumulation leading to spontaneous GREs, the actual release process must occur on fundamentally the same length and time scales as historic spontaneous GREs. Thus gob interactions resulting from the release process itself should be similar regardless of the process by which buoyancy is achieved, though there may be differences in degree. These effects, for the most part, are not included specifically in the model but are assumed to be included within the historic GRE data.

Section 2.1.1 describes proposed mechanisms that could make more gobs buoyant at the same time. Section 2.1.2 covers potential changes in the effective material strength. Changes in the shape of the void profile are discussed in Section 2.1.3; the effect of local disturbances during

the release of the first gob is treated in Section 2.1.4; and Section 2.1.5 discusses global effects on the release of the first gob. Various other issues are covered in Section 2.1.6.

\subsubsection{Mechanisms Making More Gobs Buoyant at the Same Time}

Though gas releases from each gob during decant may be indistinguishable from historic releases, the decant process may cause more gobs to release at or near the same time, causing a much larger than historical release. The most important of these mechanisms are the uncertain changes in gob size and void distribution that have occurred in the past few years due to waste cooling, as described in subsection 2.1.1.4.

\subsubsection{Lateral Void Migration}

Lateral movement of retained gas from a high-void gob into surrounding regions during depressurization by an unknown mechanism brings more gobs to near-buoyancy at the same time. 
Not included in model. This concept is incorrect. Bubbles can only move down a pressure gradient established by the surrounding fluid. In a hydrostatic pressure field, the force on a bubble is strongly upward, which would prevent significant lateral migration. Even if it could occur, lateral migration would be opposite the proposed mechanism. A locally higher void region would create a lower hydrostatic pressure due to its relative buoyancy. This pressure sink would tend to draw bubbles from lower void surroundings, making the void higher still.

\subsubsection{Faster Gas Expansion}

During decant, the average void in the tank is greater than that during natural growth because all the gas is expanding.

Included in model. While the average void increases as the hydrostatic pressure decreases, the difference in void between gobs increases because the change in void with pressure increases with void. Natural gas generation produces a constant void growth. This means that during decant the gob with the highest void will grow fastest, so the next gob in line will be relatively less buoyant when the first one goes-causing BDGREs to occur at wider intervals. The local void change with pressure is included in the enhanced model (see subsection 2.1.3.3).

\subsubsection{Bypass of Background Release}

Decant bypasses background gas release mechanisms and could make gobs buoyant that would not have been so under natural conditions.

Not included in model. This effect is inconsequential. The gobs affected by this phenomenon would become buoyant toward the end of decanting, when headspace is at a maximum and hydrostatic pressure at a minimum. This effect is not included in the enhanced model.

\subsubsection{Changes in Observed Gob Size and Void Distribution}

Changes in BDGRE behavior in the last few years, with more very small releases, may be changing the distribution of gob sizes and the relative void fraction.

Included in model. This trend increases uncertainty in historic tank behavior. Several tanks have had few or no large, historically typical gas releases in several years. However, the waste levels have stayed constant or decreased, indicating no increase in gas retention. Gob size and gas distribution could be expected to differ from earlier historically inferred parameters, but the direction is not known. A more uniform gob size and void would exacerbate gas releases. This uncertainty is included in the Monte Carlo simulation by wider input distributions on gob size and initial void.

\subsubsection{Evaporation of Dissolved Gas}

Depressurization will cause saturated dissolved gas to come out of solution into existing bubbles. This does not occur during natural void growth and would increase the void at a faster rate than depressurization alone. 
Not included in model. This mechanism is inconsequential. Ammonia is the major dissolved gas component with some contribution by nitrous oxide. However, the amount of both of these gases that is dissolved in the liquid is relatively small in the tanks to be decanted. Calculations of bubble expansion during decant based on Henry's law show that the additive effect of dissolved gas is small. This phenomenon is not included in the enhanced model.

\subsubsection{Changes in Sediment Strength}

The relatively rapid expansion of bubbles and other effects may change the effective yield stress of the sediment. This means that less over-void would be needed to enable a buoyant gob to detach than would be needed during natural void growth. However, none of the proposed mechanisms are significant.

\subsubsection{Bubble Expansion Decreases Bulk Yield Strength}

The solid-liquid matrix surrounding each bubble yields during expansion, reducing the average yield strength. This could reduce the over-void needed to make a buoyant gob break away.

Not included in model. The effect of strength is inconsequential. A lower yield stress lowers the over-void needed for gob detachment, which also reduces the energy of the release. Lower strength makes the length scale smaller, which would tend to make released gobs smaller. However, at the length scale of a gob, the effect of material strength is small and the over-void is minimal. It is also believed that the strength is reduced only in the immediate vicinity of the bubble and does not affect the bulk waste. A local reduction in yield stress would allow individual bubbles to disengage, lowering the gas content of the gob. This effect is not included in the enhanced model.

\subsubsection{Bypass Slow Relaxation}

Faster gas expansion in decant prevents slow "relaxation" of material, which effectively increases the average yield stress.

Not included in model. This effect is inconsequential. Avoiding a postulated relaxation process might increase the bulk yield stress relative to that occurring during natural GREs. This would increase the over-void required to release a gob, increasing the energy of the release and raising the potential for multiple gob releases. However, at the scale of a gob, the effect of material strength is small and the over-void is minimal. This hypothetical effect is not included in the enhanced model.

\subsubsection{Small Disturbances}

Propagation of small vibrations due to transfer pump operation could cause relative particle motion and reduce the bulk waste strength.

Not included in model. Rationale given in subsection 2.1.2.1. 


\subsubsection{Changes in Void Profile Shape Due to Depressurization}

One of the most important results of this study is the conclusion that depressurization is fundamentally different from accumulation of gas generated within the waste. The shape of the void profile is shifted slightly relative to the initial profile. While the shape change is greatest in the latter stages of decant, when headspace is at a maximum and hydrostatic pressure at a minimum, the effect is strong enough to warrant inclusion in the enhanced gas release model.

\subsubsection{Shape Independent of Gas Generation Rate}

The shape of the void profile depends on the linear viscosity profile and Stokes form for bubble velocity and is independent of gas generation rate. Therefore, assuming that gas expansion due to depressurization is equivalent to a rapid gas generation rate, GREs due to depressurization are the same as natural ones.

Not included in model. This hypothesis was later shown to be incorrect. While it is true that the void profile is independent of gas generation rate, it is affected differently by depressurization. Depressurization is not equivalent to a faster gas generation rate (see subsection 2.1.3.3).

\subsubsection{Faster Time Scale for Depressurization}

Bubble/void growth during depressurization is much faster than during natural bubble/void growth by generation and migration. The relative bubble size distribution and gas location can be considered fixed during decant.

Included in model. Difference in time scales is the basis for the enabling assumption of the model. Slow migration sets up initial conditions for a much faster decant process. Time scales are so different that gas generation and migration can be ignored during decant. This is the basic enabling assumption of the decant gas release model.

\subsubsection{Void Profile Shape Changes During Depressurization}

The peak of the void profile shifts upward during depressurization.

Included in model. The effect of pressure on void fraction increases with void and the inverse of the gas pressure according to the following expression:

$$
\frac{\mathrm{d} \alpha}{\mathrm{dP}}=-\frac{\alpha(1-\alpha)}{\mathrm{P}}
$$

Integrating Eq. (2.1) between initial state 0 and final state 1 gives

$$
\alpha_{1}=\left[\frac{\mathrm{P}_{1}}{\mathrm{P}_{0}} \frac{\left(1-\alpha_{0}\right)}{\alpha_{0}}+1\right]^{-1}
$$


The effect of Eq. (2.2) is to cause the void to increase more rapidly at higher elevations. The change in void compared with the initial profile for a hypothetical decant of 1 to $4 \mathrm{~m}$ of supernate from a tank initially with $5 \mathrm{~m}$ of supernate and $5 \mathrm{~m}$ of sediment is shown in Figures 2.1 and 2.2 for parabolic and linear void profiles, respectively. For a parabolic profile, void fraction

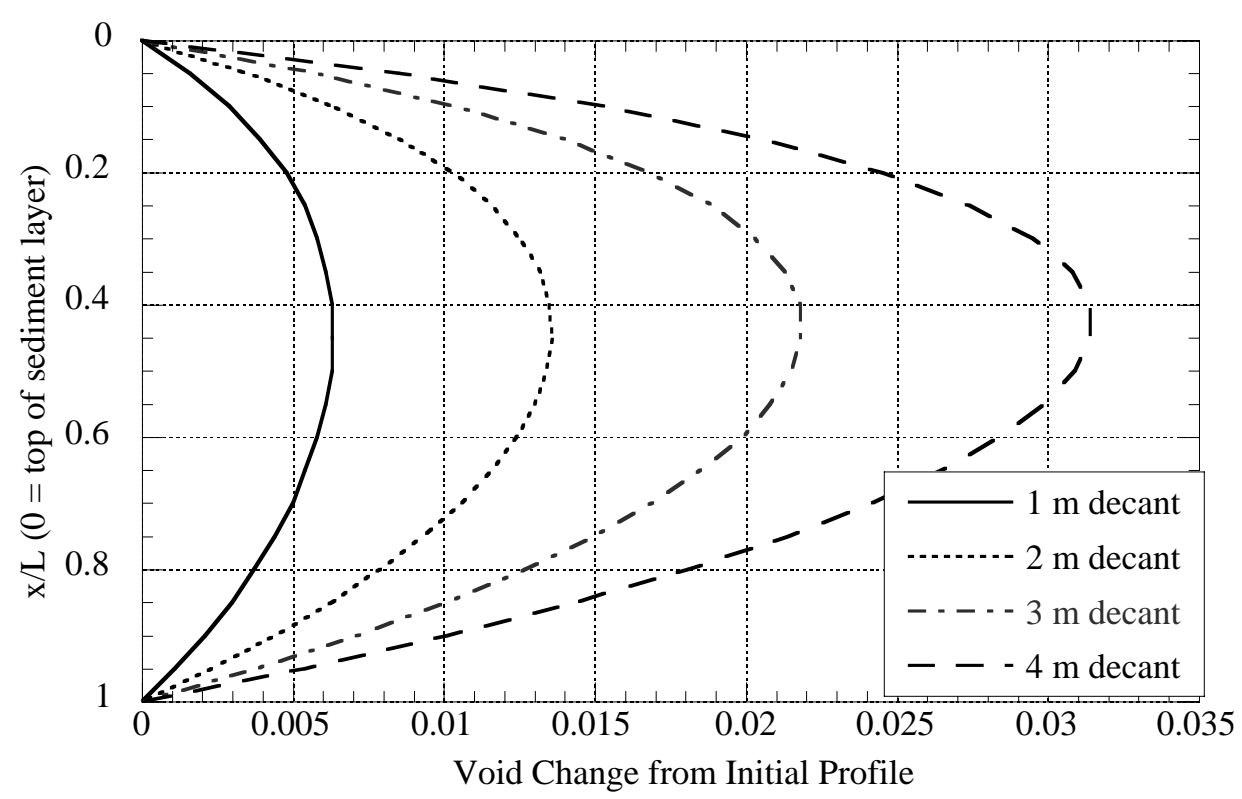

Figure 2.1. Change in a Parabolic Void Profile from Initial Profile During Decant

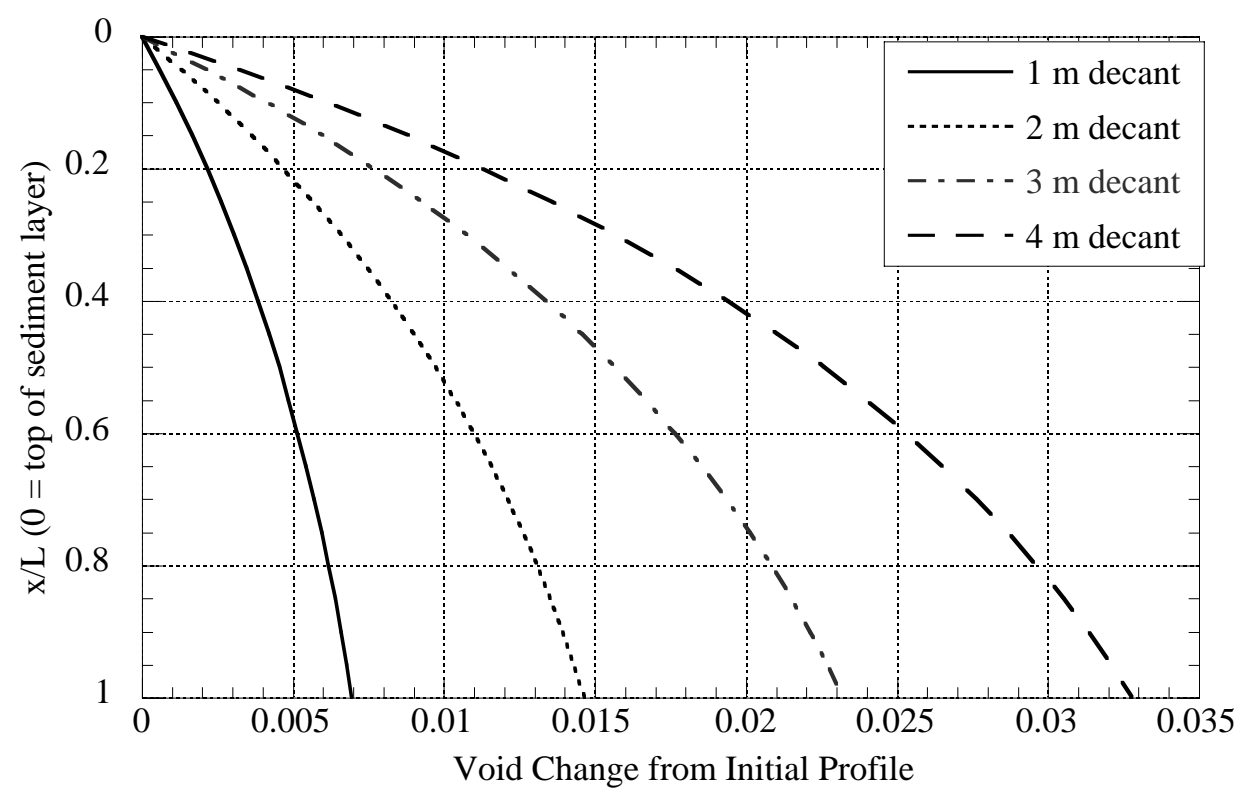

Figure 2.2. Change in a Linear Void Profile from Initial Profile During Decant 
above the peak increases faster, causing the peak to shift upward slightly and reducing the depth of material that becomes buoyant. Also, the average void computed from the void profile increases faster than the effect of the average pressure decrease on the initial average void. For a linear profile, the upper portion also grows more rapidly, but the maximum void remains at the bottom. The average of the linear profile lags the effect of pressure on the initial average void, opposite the behavior of the linear profile.

\subsubsection{Local Disturbance During Release}

The mechanisms in this section all relate to the effects of local disturbances around a releasing gob. It is recognized that the detachment and rise of an over-buoyant gob releases a large amount of potential energy in a few minutes. The fluid motion around a rising gob several meters in diameter can produce large hydrodynamic loads that can move a significant volume of sediment. Also, breaking the forces that were restraining the buoyant gob alters the static force balance of the remaining gobs.

While some of these effects could potentially lead to multiple gob releases or exacerbate single-gob releases, there is no fundamental difference in the dynamics of releases resulting from natural gas accumulation or from depressurization during decant. Therefore, no attempt is made to model any of the specific mechanisms proposed in this section. Their effects are evidenced in observed variation of gas release volume and are included in the gob size and void distributions input to the Monte Carlo simulation.

\subsubsection{Released Gob Was Restraining an Adjacent Gob}

When the buoyant gob releases, part of the restraint on unreleased gobs is removed.

Not included in model: This hypothesis was later shown to be incorrect. Because the primary gob is over-buoyant, it is restrained by adjacent material prior to release. This means that the force it exerts on adjacent gobs is upward, and release of this gob represents a relaxation of the upward force, not removal of a restraining force. The net effect is opposite the proposed mechanism.

\subsubsection{Stacked Gobs}

Release of a part-depth gob causes another gob below it to release.

Not included in model: This hypothesis is implausible. Both gobs would need to be buoyant for both to be released. If both are buoyant, they would act as a single gob. Also, both gobs going buoyant simultaneously would have the same effect as one nominal-sized one.

\subsubsection{Backflow of Supernate Removes Overburden}

When a gob is released, liquid flows back into the volume it occupied in the sediment. This inflow could sweep overburden off adjacent gobs to make them buoyant. 
Not included in model: The upper portion of the sediment is weakest and most subject to disturbance by flow of supernate. Flow velocities are such that movement of the overburden is minimal. Further, adjacent gob would need to be nearly buoyant to release. This behavior, if real, is implicitly included in the model through the uncertainty distributions describing historical multiple BDGRE behavior.

\subsubsection{Overburden Sloughs into the Cavity Left by Rising Gob}

Weak material flows to backfill the released gob. This removes overburden to make adjacent gobs buoyant.

Not included in model: Same as subsection 2.1.4.3.

\subsubsection{Spent Gob Resubmergence}

After gas release at the waste surface, it is assumed that a spent gob sinks more or less intact back onto the sediment. When the spent gob contacts the sediment layer, the disturbance could move overburden off another gob so it becomes buoyant.

Not included in model: This hypothesis is implausible. Assuming the spent gob is essentially intact, the outflow of liquid between the falling gob and the sediment would sweep away some of the weak overburden. However, the overburden is immediately replaced by the spent gob, which is probably more negatively buoyant than the overburden it replaces. Also, submergence should be far less energetic than release because the gas content of the falling gob averages less than half of neutral buoyancy.

\subsubsection{Releasing Gob Lifts Adjacent Sediment}

All or part of an adjacent nonbuoyant gob is pulled up, reducing hydrostatic pressure to make it buoyant also.

Not included in model: By definition, a body of sediment that breaks free of the sediment and rises as a single entity is a gob. While a gob must be buoyant on the average, it contains portions that are negatively buoyant and parts that are over-buoyant. The proposed mechanism actually describes the release of a single gob. This behavior is implicitly included in the model through the uncertainty distributions describing historical multiple BDGRE behavior.

\subsubsection{Global Disturbance During Release of First Gob}

In a global disturbance, release of one gob affects all the remaining waste. This is one area where the decant rate may have an influence, especially for mechanisms involving suspended particles. The effect will be stronger if the period between depressurization-induced GREs is less than the time required for particles to settle out. The most important of these is the reduction of the neutral buoyancy void fraction described in subsection 2.1.5.5. Essentially all of these mechanisms are included in the enhanced decant gas release model. 


\subsubsection{Suspended Solids Increase Hydrostatic Pressure}

Suspended solids from prior releases increase the density of the convective layer, which increases the hydrostatic pressure on the nonconvective layer. This pressure increase counteracts some of the expansion from decanting and delays release of subsequent gobs.

Included in model: An increase in hydrostatic pressure compresses stored gas and reduces the void fraction to prevent a gob from becoming buoyant. To evaluate the potential pressure increase, assume an entire gob is suspended in the supernate and that the total volume of supernatant liquid increases by the volume of the gob (i.e., backfills the "hole" left by the suspended gob). Under this scenario, the new density can be calculated by

$$
\rho_{\mathrm{CL} 1}=\frac{\mathrm{H}_{\mathrm{CL}} \mathrm{A} \rho_{\mathrm{CL} 0}+\mathrm{V}_{\mathrm{gob}} \rho_{\mathrm{gob}}}{\mathrm{H}_{\mathrm{CL}} \mathrm{A}+\mathrm{V}_{\mathrm{gob}}}
$$

where $\mathrm{H}_{\mathrm{CL}}$ is the supernatant depth and $\mathrm{A}$ is the tank area. For a large gob, $10 \mathrm{~m}$ in diameter and $5 \mathrm{~m}$ deep, with a density of $1,600 \mathrm{~kg} / \mathrm{m}^{3}$, suspended in $4 \mathrm{~m}$ of supernate with an initial density of $1,400 \mathrm{~kg} / \mathrm{m}^{3}$, the new density is $1,438 \mathrm{~kg} / \mathrm{m}^{3}$. This raises the pressure beneath $4 \mathrm{~m}$ of fluid from $1.542 \mathrm{~atm}$ to $1.557 \mathrm{~atm}$, a change of $1 \%$, equivalent to $11 \mathrm{~cm}(4.3 \mathrm{in}$., about 12,000 gal) of the original supernate. Using Eq. (2.2), this pressure change causes less than a 1\% reduction in an initial void fraction of 0.10 . Though this is insignificant, the effect of solids suspension on the supernate density and hydrostatic pressure is included in the enhanced model.

\subsubsection{Gas Release Reduces the Hydrostatic Head}

The convective layer depth drops slightly as liquid backfills the "hole" left by the released gas. The corresponding reduced hydrostatic pressure increases the void in remaining gobs.

Included in model: To evaluate this phenomenon, consider the release of all the gas in the large gob described in subsection 2.1.5.1. The 10-m-diameter, 5 -m-thick gob contains $39 \mathrm{~m}^{3}$ of gas in situ, assuming a void fraction of 0.10 . Release of this volume would lower the convective layer by $9.5 \mathrm{~cm}$. This would result in a hydrostatic pressure reduction of only $0.8 \%$, which increases the void fraction by about the same amount. Though insignificant, the effect of gas release on the convective layer volumes and the hydrostatic pressure is included in the enhanced model.

\subsubsection{Suspension of Solids Increases Supernate Viscosity}

The increased supernate viscosity could change the size and release characteristics of subsequent gobs.

Not included in model: In classic Rayleigh-Taylor instability, the viscosity difference between layers has a strong influence on the wavelength of the disturbance. In the approximate stability analysis developed to predict the gob diameter (Meyer et al. 1997), the supernate viscosity was assumed small, and the gob volume was ultimately predicted to be proportional to 
the sediment yield stress as an approximation of the sediment viscosity. Thus changes in supernate viscosity theoretically do not affect the gob size. This is consistent with the assumption that the decant process acts on the gob size and void fraction established by the preceding steadystate period. Supernate viscosity changes are not explicitly included in the enhanced model, but the distribution of gob sizes generated from the historical BDGREs can be said to implicitly cover the potential effects.

\subsubsection{Suspension of Solids Reduces Average Overburden}

Sediment suspended in the convective layer from prior releases during decant reduces the average overburden on the nonconvective layer, allowing other gobs to release sooner.

Not included in model: This postulation is not correct. Actual, not average, overburden is what determines gob buoyancy. Settling of suspended solids from prior BDGREs would actually add to existing overburden of gobs that have not yet gone. The added overburden would tend to delay their buoyancy slightly. However, this effect is small compared with the effect on neutral buoyancy void fraction discussed in subsection 2.1.5.5. This effect is not included in the enhanced model.

\subsubsection{Suspension of Solids Reduces the Neutral Buoyancy Void Fraction}

Suspension of solids increases the density of the convective layer, which reduces the neutral buoyancy void fraction. Subsequent gobs can therefore be released.

Included in model: Suspended sediment has a strong effect on fluid density. For the example given in subsection 2.1.5.1, an increase in supernate density from 1,400 to $1,438 \mathrm{~kg} / \mathrm{m}^{3}$ with a sediment density of $1,600 \mathrm{~kg} / \mathrm{m}^{3}$ changes the neutral buoyancy void fraction $\left(\alpha_{\mathrm{NB}}=1\right.$ $\rho_{\mathrm{CL}} / \rho_{\mathrm{CL}}$ ) from 0.125 to 0.10 , a reduction of $20 \%$. Of course, the entire gob is not suspended but some smaller fraction, possibly 1 to $10 \%$. However, if several gobs are released and a significant fraction of their solids suspended early in decant, the neutral buoyancy void fraction for subsequent gobs would be lowered. There is the potential for all releasable gobs to be released in a rapid sequence. The effect of solids suspension is included in the enhanced model. The fraction of solids suspended is varied in the analysis.

\subsubsection{Other Decant Issues}

Some of the phenomena in this section did not fit into the categories of prior sections. They include thermal effects and some potential changes in the dynamics of buoyant instability.

\subsubsection{Slow Thermal Relaxation}

The time scale for thermal relaxation is much longer than that of gas expansion. Thus the temperature profiles of gobs about to be released may be different during decant than during natural growth. 
Not included in model: Temperature differences are small. The largest is in AN-104, where the nonconvective layer temperature ranges from $80^{\circ}$ to $100^{\circ} \mathrm{F}$. Based on observations of temperature changes in response to natural GREs (Hedengren et al. 2000), release of a gob would expose surrounding unreleased gobs to the lower temperature. Using PV/T = constant, the 20-degree temperature change would reduce the gas volume by $4 \%$. However, the thermal mass of the waste is very high, and this temperature change would require weeks or months to reequilibrate-much longer than the entire decant process. Lateral temperature nonuniformity effects are not included in the model.

\subsubsection{Nonequilibrium Thermal Profile}

The changing temperatures in resettled gobs could affect release behavior of nearby unreleased gobs.

Not included in model: Same as subsection 2.1.6.1.

\subsubsection{Atmospheric Pressure Changes}

Barometric pressure fluctuations are observed to trigger some GREs that are about ready to release. This effect should approximate the initial stages of decant because the time scales of the pressure change are similar.

Not included in model: This is a potential source of information. The GRE histories of the tanks under consideration can be checked to determine whether GREs that were triggered by a pressure change are different in any significant way from others. However, it is not clear how the results of such a study could be scaled up to the decant process.

\subsubsection{Decreasing Supernate Depth}

Besides reducing the gob potential energy, lowering the supernate may change the dynamics of gob release behavior.

Included in model: As the supernate depth decreases, the hydrostatic pressure on the gas decreases, causing the void in the sediment layer to increase. This is the basic trigger for BDGREs during decant. However, the fraction of the gas that is released also decreases with the hydrostatic pressure by $\mathrm{F}=\left(1-\mathrm{P}_{0} / \mathrm{P}_{\mathrm{GAS}}\right)$. The release fraction is $50 \%$ for $\mathrm{P}_{\mathrm{GAS}}=2$ atm but only $33 \%$ for $\mathrm{P}_{\mathrm{GAS}}=1.5 \mathrm{~atm}$. The potential energy available to cause gas release decreases nonlinearly with supernate depth such that the later gobs may not release gas at all. These effects were in the original model and are retained in the enhanced model.

\subsubsection{Dynamics of Buoyant Instability}

The natural maximum and minimum unstable gob size may change with depressurization because it is so much more rapid than the natural process. 
Not included in model: This hypothesis was later shown to be implausible. The gob size expression developed from an approximate Rayleigh-Taylor stability analysis does not depend on hydrostatic pressure or on the height of the supernatant liquid layer. It does, however, depend weakly on the neutral buoyancy void fraction, which may decrease after the first GRE (see subsection 2.1.5.5). Therefore, the gob size is expected to be the same for the first event but may change slightly in subsequent releases. The difference is expected to be far less than the uncertainty in the gob size. This is implicitly covered in the analysis, so the potential effect is not included explicitly in the enhanced model.

\subsubsection{Decant Slowly to Match Natural Time Scale}

The lower flow rates ( 5 to 10 gpm) of current saltwell pumps would perform a decant in months rather than in days. This would more closely approximate the natural time scale.

Investigated: The difference between spontaneous GREs and those induced by decant is more than a matter of time scale alone. It was found that void growth by depressurization of existing gas is fundamentally different from accumulation of gas generated in the waste, though the effect is small for gas fractions of interest. To the extent it accommodates these differences, the enhanced decant gas release model can assess whether much slower decant rates would be advantageous. A sensitivity study showed that the predicted headspace hydrogen concentration is not sensitive to decant rate (Section 3).

\subsection{Phenomena Affecting Initial Mixer Pump Operation}

Mixing the waste in a tank will release most of the gas stored therein. The desire is to operate the mixer pumps initially so that gas is released in a controlled, predictable manner. Strategies to resolve complications of deploying the mixer pumps may partially degas the tank before the mixer pump is operated. For example, supernate decant will make it easier to start and run the pump. Specific degassing strategies will need to be modeled when defined. The current modeling effort assumes a degassing scenario similar to SY-101 but using the two W-211 baseline pumps (with bottom inlet) that run in a fixed azimuth one at a time for a relatively short duration. To plan this operation, the ability of these large mixer pumps to mobilize waste needs to be modeled as does the potential for waste mobilization to induce additional gas releases.

Unlike supernate decanting, where BDGREs are induced by depressurization, the dominant gas release mechanism in mixing is expected to be mobilization of the gas-bearing waste by the jet. The concern is that BDGREs may also be induced that add to the gas released by mixing. Section 2.2.1 addresses direct effects of the jet, while Section 2.2.2 discusses implications of solids suspended by mixing. Section 2.2.3 covers other, nonspecific issues.

\subsubsection{Effects of the Jet}

The details of mobilizing a sediment bed by a mixer pump jet are not known. However, transient computational fluid dynamics simulations show it to be a complex process of repeated 
tunneling and collapse that affects a relatively narrow region. This process may induce gas releases or aggravate those already induced by other mechanisms, as discussed in this section.

\subsubsection{Differences in Mixer Pump Design}

The degassing behavior of the baseline W-211 pump with bottom suction may be significantly different than that of the SY-101 pump with high suction.

Not included in model: Bottom suction will tend to produce a neutrally or negatively buoyant jet rather than a buoyant jet as in SY-101. Data show that the buoyancy of the SY-101 jet had a significant effect on the penetration distance. Also, the high inlet forces the fluid expelled by the jet to circulate upward, creating a significant lifting force in some situations; the bottom inlet cannot create this kind of "piston" lifting effect. Other geometric factors may have as powerful an effect in degassing as the inlet location. However, the only useful data from an actual degassing operation came from SY-101, and there are no data to compare this with a similar operation with a bottom-inlet pump. Therefore, while jet buoyancy was considered in developing the model, it was not specifically included in the final form.

\subsubsection{Mechanical Agitation}

If a BDGRE is induced during mixing, the release fraction of that gob could be larger due to mechanical agitation if the rising gob is in the area of the jet.

Not included in model: A GRE induced by reduction in the neutral buoyancy void fraction due to solids suspension is assumed to release gas until it is neutrally buoyant at the surface. This effect is included in the model, but no additional gas release is attributed to fluid agitation. The gob would be rising out of the region of influence of the jet, the region of influence of the jet is relatively small, and the time the rising gob might be exposed to additional turbulence is also small. However, the model includes a reduction in gob volume before it becomes buoyant if it is in the path of the jet.

\subsubsection{Undermining}

The jet penetrates the waste and undermines an area, lifting it up and causing it to become buoyant.

Not included in model: Undermining is unlikely. Recent computational simulations show that the jet is more likely to break up the material than to lift it as a coherent body. The bottom inlet of the mixer pump prevents hydraulic lifting, and gobs can be lifted only by dynamic pressure. Also, several factors reduce or prevent significant gas release from a lifted gob: 1) the fraction of gas released in a spontaneous buoyant GRE is less than would be released if the jet disrupted the same volume, 2) some of the gas in a lifted gob would be released in the process of undermining, 3) if a nonbuoyant gob is lifted mechanically so it becomes buoyant at a higher elevation, less energy is released to yield the waste, resulting in a low or negligible gas release. In the limit, a postulated multigob lifting event is similar to supernate decant. Undermining is not included in the model. 


\subsubsection{Effect of Off-Center Pumps}

The two pumps are displaced from the tank center, so one jet will always be impinging on the tank wall (if run enough for a sufficiently long duration).

Not included in model: It is unlikely that jet would erode waste effectively after impingement. However, the model conservatively assumes both jets have equal effect.

\subsubsection{Suspended Solids Effects}

As was noted in Section 2.1, suspension of solid particles in the supernate is a very important factor in inducing BDGREs via decreases in the neutral buoyancy void fraction. This is also a likely occurrence in mixing as mentioned in subsection 2.2.2.2. Some other effects pertaining to solids are also covered in this section.

\subsubsection{Increased Supernate Viscosity}

Increases in supernate viscosity due to solids suspended by mixing and/or gas release may change release behavior.

Not included in model: The effect of higher viscosity would absorb more energy as the gob rises and thus tends to be moderating rather than exacerbating. Also, gob rise time is short regardless of changes in viscosity.

\subsubsection{Reduction in Neutral Buoyancy Void Fraction}

Mixer pump operation suspends solids, increasing the convective layer density, and reducing the neutral buoyancy void fraction.

Included in model: Solids suspension is a strong effect, as discussed in Section 2.1. The decreased neutral buoyancy void fraction due to solids suspension from both mixing and other induced GREs is included in the model.

\subsubsection{Dissolution of Solids}

Dissolution may occur during mixing if the supernate is not in equilibrium with the sediment. Dissolution of solids could broaden the region of influence of the jet, release more gas, and increase the density of the supernate more than suspension alone.

Not included in model: Tanks that have experienced BDGREs, which are of interest in this study, have been stirred periodically by these events over many years so are probably in equilibrium, at least in the major soluble species. No dissolution effects are included in the model. 


\subsubsection{Other Mixing Issues}

This section contains two suggestions that fell outside the preceding categories.

\subsubsection{Alternative Parameters}

The degassing plan should be based on something other than energy input (e.g., effective cleaning radius, momentum).

Not included in model. The degassing plan is not based on parameters such as effective cleaning radius because basic scaling laws demand that the model depend on energy input.

\subsubsection{Consideration of LANL Safety Analysis}

Many exacerbating mechanisms associated with mixing were considered in the Los Alamos National Laboratory Safety Analysis for SY-101 mixing (Sullivan 1995). This document should be reviewed.

Not included in model: The SY-101 LANL Safety Analysis basically ignores detailed mixing and gas release mechanisms, and the major assumption was that mixer pump operation would cause a large GRE. The GRE size was correlated to waste level by extrapolating historic GREs without considering specific mixer pump effects. The document was reviewed and none of the exacerbating mechanisms discussed in the report could be suitably incorporated into the enhanced mixing model.

\subsection{Reanalysis of Historical Gas Release Data}

To better define the distribution of gob sizes and interaction, a much more detailed analysis was conducted of historic gas releases. This section describes the analysis of GREs in tanks AN-103, AN-104, AN-105, and AW-101, recorded by headspace gas monitoring equipment since 1994-1995 (McCain 2001). While these data have been analyzed before, only recently have we recognized that many of the events consist of multiple releases occurring relatively close together over a comparatively short time. Headspace hydrogen concentration data for each release event were reviewed to determine whether more than one gob was released. For multigob events, the data were parsed into discrete subevents describing the release of each participating gob. The additional depth of understanding provided by these analyses significantly strengthened the technical basis for the parameter values used for modeling BDGREs during waste retrieval activities.

It was also recognized that the original conservative assumption of instantaneous gas releases (Section 1) was not physically realistic. Using the same rate function used to fit the hydrogen concentration data, gas releases were modeled as functions of time occurring over hours, as observed in the data. This same rate function was used to calculate the rate of suspension of gob material into the supernate. See the model description in Section 3.1 and the parameter definitions in Section 3.2 for a complete description of these derivations. 
The gas release data were parsed into their individual components by applying the exponential gas release model described in Hedengren et al. (2000), which was derived by observing a large number of events. In this model, the hydrogen release rate is assumed to follow the form

$$
\mathrm{Q}_{\mathrm{R}}=\mathrm{Q}_{\mathrm{R} 0}\left(\frac{\mathrm{t}-\tau_{0}}{\tau}\right) \mathrm{e}^{-\left(\frac{\mathrm{t}-\tau_{0}}{\tau}\right)}
$$

where

$$
\begin{array}{ll}
\mathrm{Q}_{\mathrm{R} 0} & =\text { hydrogen release rate constant } \\
\tau & =\text { time to peak release rate } \\
\mathrm{t} & =\text { current time } \\
\mathrm{t}_{0} & =\text { time at which the release started. }
\end{array}
$$

The model uses this release rate in the continuity equation for headspace hydrogen,

$$
\mathrm{V}_{\mathrm{HS}} \frac{\mathrm{d}\left[\mathrm{H}_{2}\right]}{\mathrm{dt}}=\mathrm{Q}_{\mathrm{R}}-\mathrm{Q}_{\mathrm{V}}\left[\mathrm{H}_{2}\right]
$$

where

$\mathrm{Q}_{\mathrm{R}} \quad=$ model hydrogen release rate [from Eq. (2.4)]

$\mathrm{Q}_{\mathrm{V}} \quad=$ headspace ventilation rate

$\mathrm{V}_{\mathrm{HS}} \quad=$ headspace volume

$\left[\mathrm{H}_{2}\right] \quad=$ hydrogen concentration ${ }^{(2)}$ in the headspace.

The relationship in Eq. (2.5) is valid as long as $Q_{R}<<Q_{V}$, which is the case for even the largest GREs. The headspace volume and ventilation rate are assumed constant over the duration of the event. There is also the implicit assumption that the hydrogen gas released in the event very quickly becomes well mixed in the headspace. The hydrogen concentration measurements are therefore assumed to represent the entire headspace. A large body of computational and experimental evidence has shown that this is a reasonable assumption (Huckaby et al. 1997).

The model is applied by solving the system of these two equations iteratively, adjusting the parameters $\mathrm{Q}_{\mathrm{R} 0}$ and $\tau$ to minimize the cumulative root-mean-square error between the calculated and measured headspace hydrogen concentration. If the headspace ventilation rate, $\mathrm{Q}_{\mathrm{V}}$, is not known, it must also be solved for in the iteration process, subject to the constraint of a reasonable range of possible values and based on physical conditions in the tank and associated ventilation system. When a set of values has been determined that gives an acceptable fit to the hydrogen concentration measurements for the event, the total gas release volume due to the GRE can be calculated from the hydrogen release as

(a) The peak hydrogen release rate is equal to $\mathrm{Q}_{\mathrm{R} 0} \mathrm{e}^{-1}$.

(b) This term is actually a volume fraction, with units of $\mathrm{m}^{3}$ of hydrogen gas over $\mathrm{m}^{3}$ of release gas, but to preserve historical continuity with earlier publications of the model, it is referred to here as a concentration. 


$$
\mathrm{V}_{\mathrm{R}}=\frac{1}{\chi_{\mathrm{H}}} \int_{\mathrm{t}_{0}}^{\infty} \mathrm{Q}_{\mathrm{R}} \mathrm{dt}=\frac{\mathrm{Q}_{\mathrm{R} 0} \tau}{\chi_{\mathrm{H}}}
$$

where $\chi_{H}$ is the fraction of hydrogen in the gas retained in the waste.

For GREs where the measured headspace hydrogen concentration (in ppm) consists of the typical sharp rise followed by an exponential decay, this model does a very good job of characterizing the event. The model fits the changing concentration with time, and provides a good estimate of the total hydrogen release volume. Figure 2.3 illustrates this approach for what is clearly a single-release event in AN-104 on October 2, 1995. This event occurred before flow controllers were installed on the tank ventilation system, so the headspace ventilation rate was not precisely known. It was therefore solved for as part of the iterative process of fitting the model to the data. The estimated ventilation rate obtained for this event was $92 \mathrm{scfm}$, which is a reasonable value for the conditions in the tank at that time.

Since inlet flow controllers were installed in the ventilation systems of the AN farm DSTs in mid-1996, the headspace ventilation rate for AN-103, AN-104, and AN-105 has been maintained at an essentially constant nominal rate, $100 \mathrm{scfm}$. Figure 2.4 shows a single-release GRE that occurred in AN-105 in 1999. The calculated hydrogen concentration determined with the model is in very good agreement with the measured concentration values. When fitting the model to this event, the headspace ventilation rate was assumed to be constant at the nominal value of 100 scfm, and only the model coefficients $Q_{R 0}$ and $\tau$ were solved for in the iterative process.

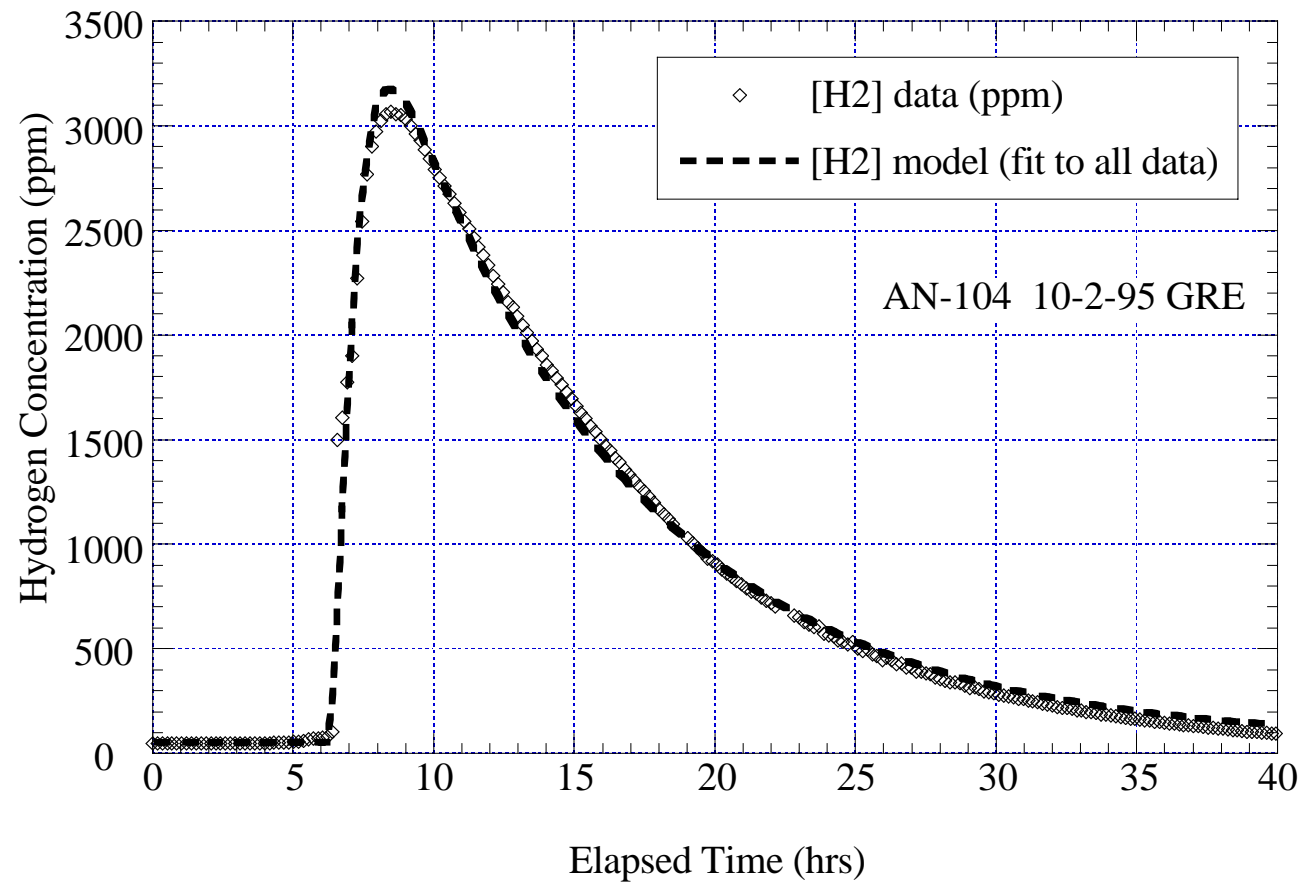

Figure 2.3. GRE in AN-104 on 10/2/95, Comparing Calculated and Measured Headspace Hydrogen Concentrations (ventilation rate unknown; solved for in model application) 


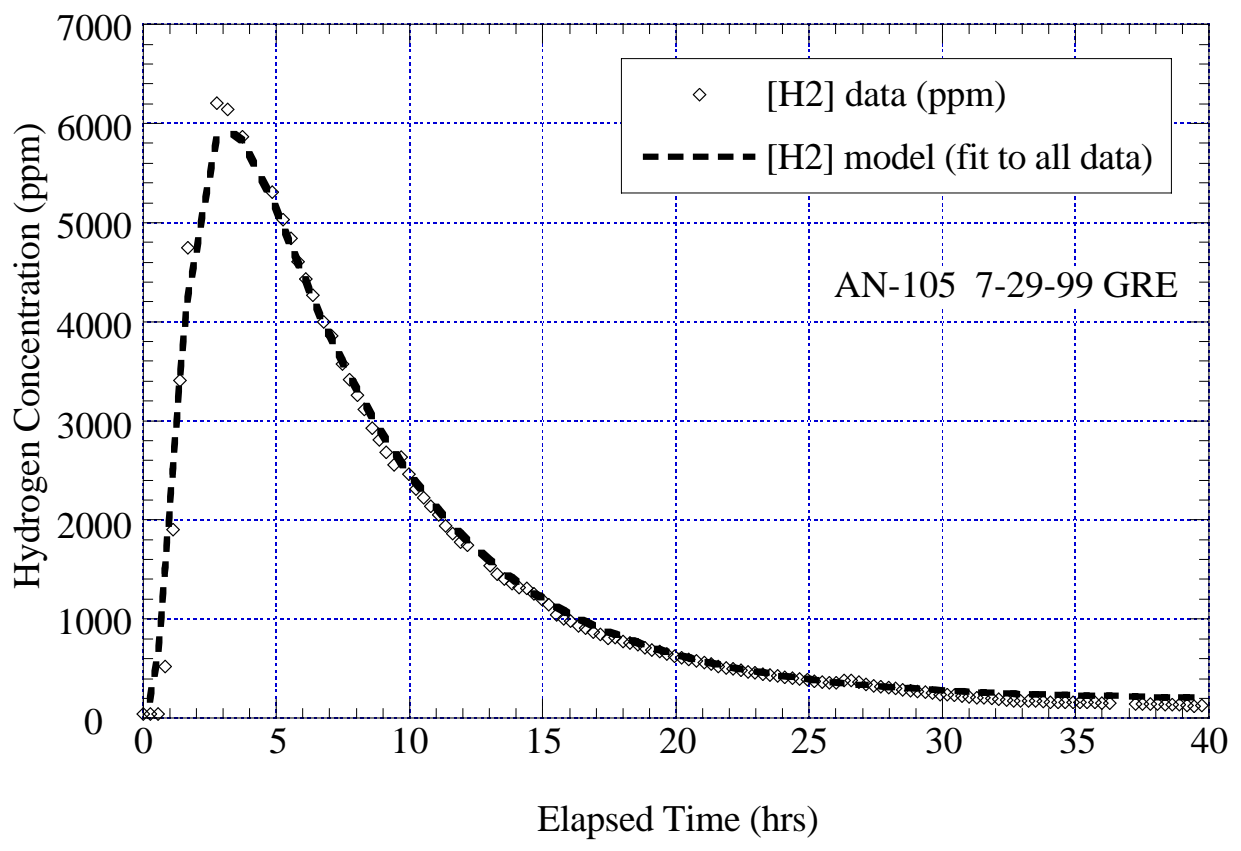

Figure 2.4. GRE in AN-105 on 7/29/99, Comparing Calculated and Measured Headspace Hydrogen Concentrations (ventilation rate known, assumed constant at nominal value of $100 \mathrm{scfm}$ )

Examination of the headspace hydrogen concentration data from tanks AN-103, AN-104, AN-105, and AW-101 shows that GREs in many cases consist of multiple release events. Figure 2.5a shows a number of such releases from two different tanks. The shapes of the hydrogen concentration curves indicate that, in this relatively small sample of multiple-release events, the GREs can consist of two, three, four, or more individual releases. A simple generic model would be unlikely to fit all of these different GREs, even though the underlying events are of the same general character. Further, it is difficult to determine the number of BDGREs in an event until the data are parsed into discrete subevents describing the release of each participating gob.

As an example, if the gas release model is applied to the event in AN-104 on 6-2-00, treating it as a single release, the "best fit" is that shown by the dashed line in Figure 2.5b. This event consisted of a sharp peak in hydrogen concentration (to $264 \mathrm{ppm}$ ), followed by an exponential decay interrupted for a time with a sustained 'hold' at about $200 \mathrm{ppm}$. Because the ventilation rate is essentially constant and the calculated hydrogen concentration "misses" the time of the peak value and only roughly approximates the shape of the data curve, there is clear evidence that there has been additional gas release since the initial event.

An alternative approach to analysis of such multiple release events has been developed that uses the principle of superposition to parse out the series of gas releases making up the GRE. The model can very accurately represent the peak hydrogen concentration and the exponential decay of the hydrogen concentration as headspace ventilation clears out the released gas and returns to the normal 'background' hydrogen concentration for the tank, and does so very well for those events consisting of a single BDGRE. It is therefore reasonable to suppose that a good 


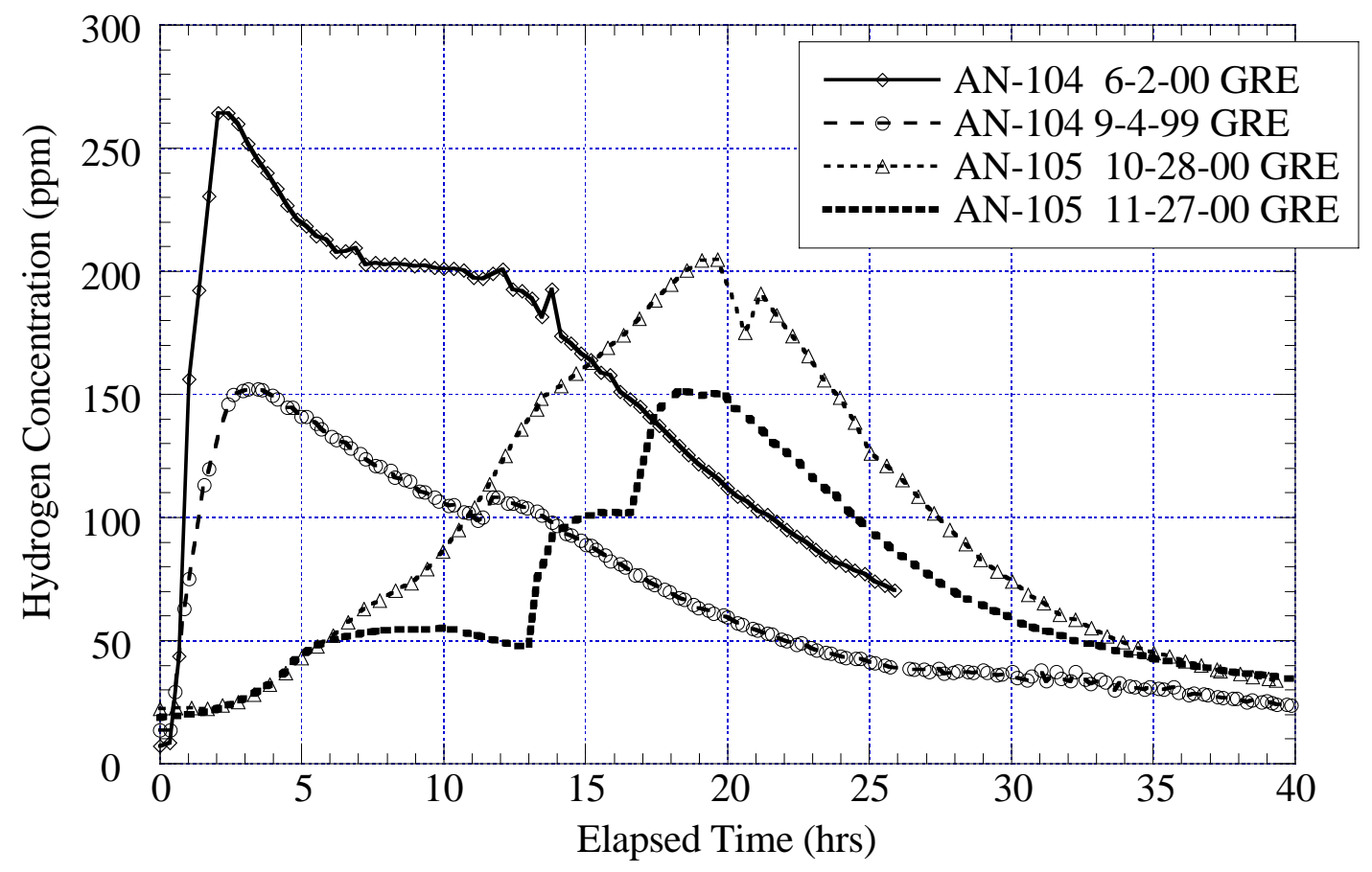

Figure 2.5a. Examples of GREs that Consist of Multiple Releases

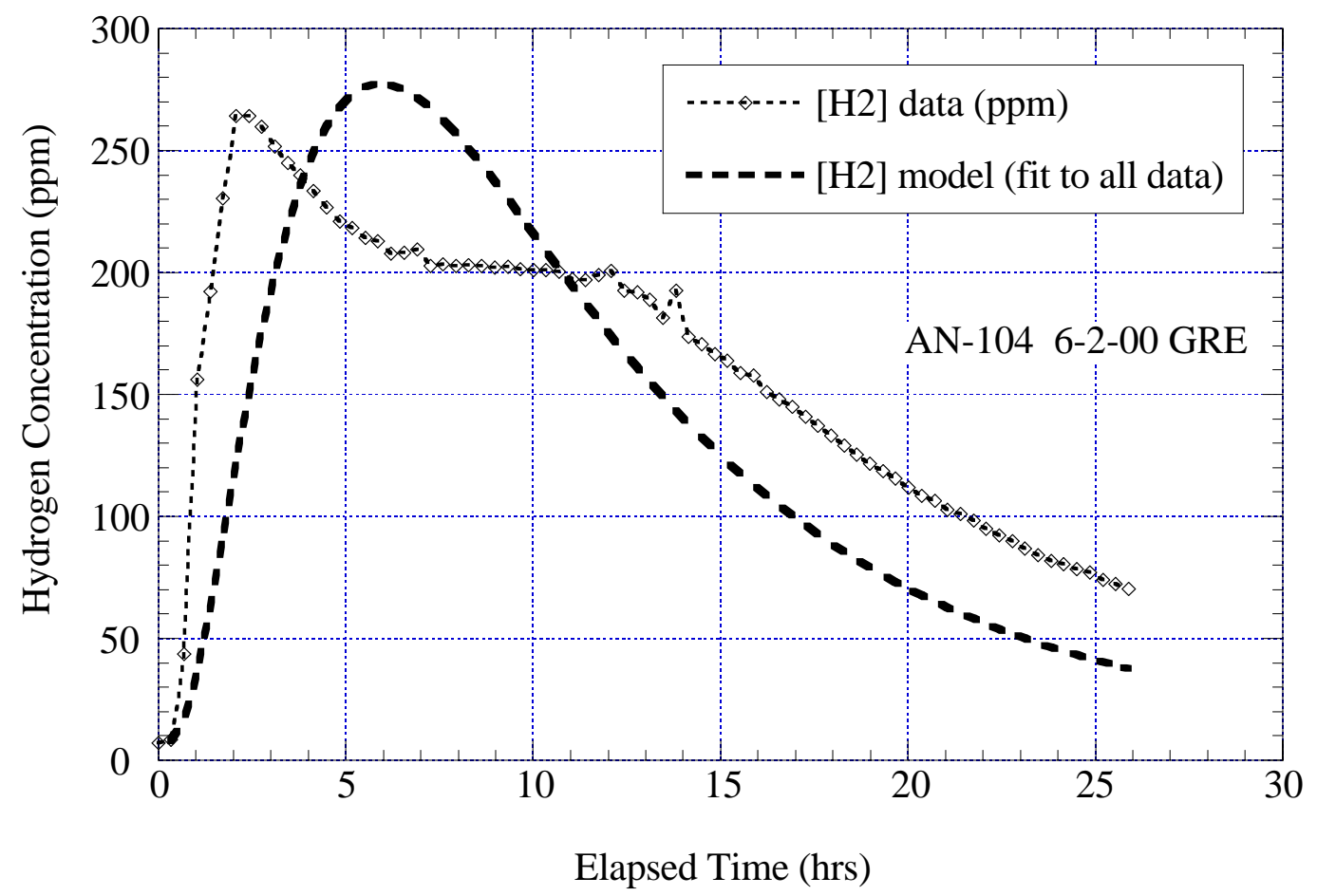

Figure 2.5b. GRE in AN-104 on 6-2-00, Showing Model Fit to All Data Without Differentiation of Separate Releases 
estimate of the shape of a subsequent release can be obtained by using the model to estimate the shape of the hydrogen concentration curve for the first release and subtracting that estimated concentration from the measured concentration data. This yields a new curve of hydrogen concentration that is an estimate of the "real" shape of the second release of the event.

Figure 2.6 shows the results obtained with this approach for the multiple-release GRE in AN-104 on June 2, 2000. Instead of fitting the model to the entire data set, the model is initially fit only to the "first peak" of the release, as indicated by the plot of the data during the first five hours of the event. As shown by the short-dashed line in Figure 2.6, the model does an excellent job of fitting this part of the release when constrained to consider only the first five hours of data. Applying the model in this manner yields an estimate of the volume of gas released by the initial release of the event and a predicted concentration history of what was expected to occur had this been the only release during the event.

The curve describing the model prediction for this "first peak" can be used to obtain an estimate of the concentration that would have been obtained in the headspace for the second release, had it occurred on its own. That is, the "real" second peak can be described by the relationship

$\left[\mathrm{H}_{2}\right]_{\text {real' 2nd peak }}=\left[\mathrm{H}_{2}\right]_{\text {data }}-\left[\mathrm{H}_{2}\right]_{\text {predicted 1st peak }}$

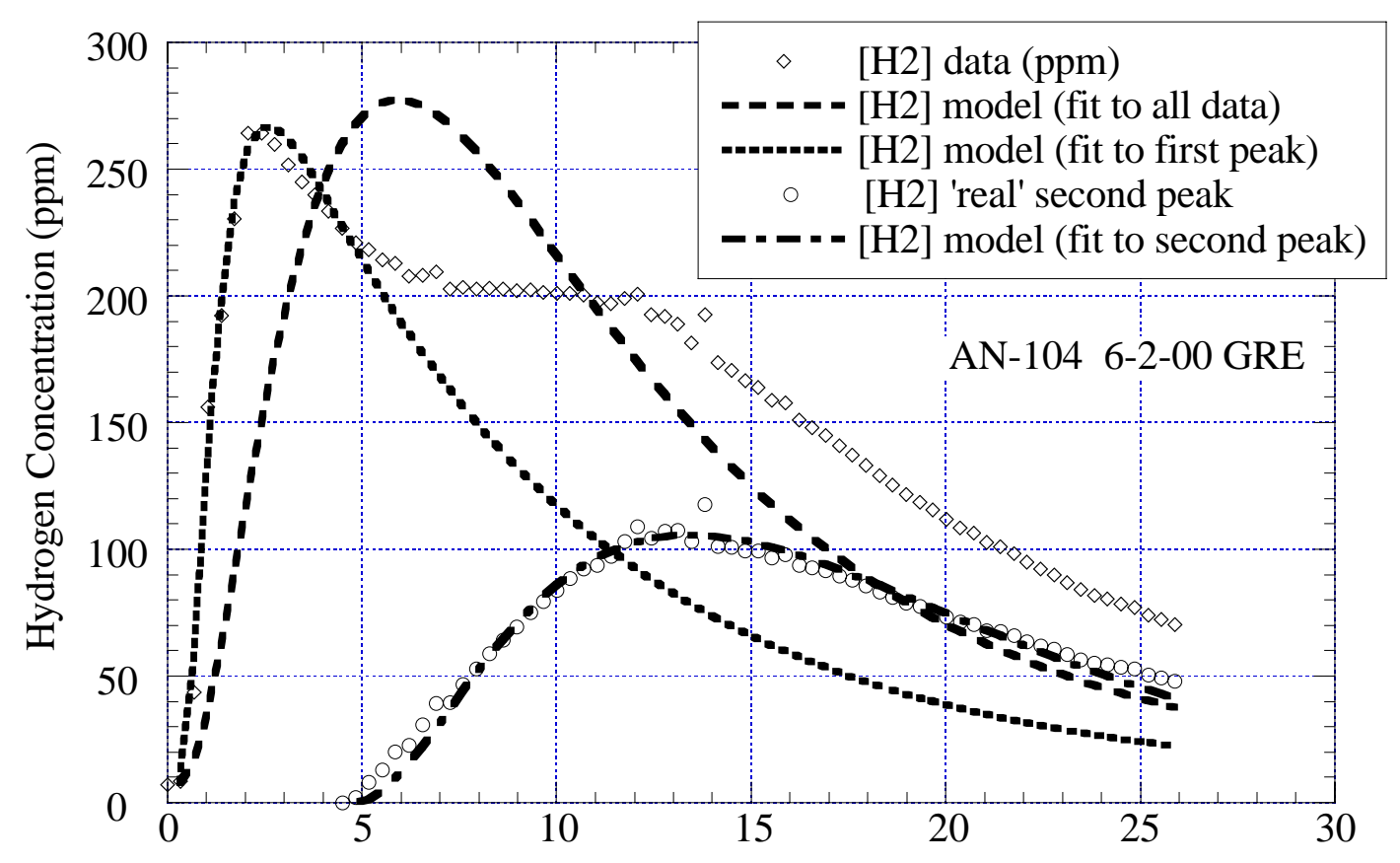

Elapsed Time (hrs)

Figure 2.6. GRE in AN-104 on 6-2-00, Parsed into Multiple Releases 
The model can be fit to the estimate of the second "real peak" obtained using Eq. (2.7), yielding an estimate of the volume of gas released by the second release of the GRE and a predicted concentration history for the decay of this "second peak" of the event. For the GRE in AN-104 on 6-2-00, the event appears to parse out into only two separate peaks. However, this process of deconstructing the event as a superposition of releases can be repeated as many times as necessary to uncover all of the significant individual releases in a multiple-release GRE. The total hydrogen gas release for the event is estimated by summing the volumes calculated for the individual events. That is, the total release rate for a multiple-release event can be estimated by summing the contributions of its components via Eq. (2.4) as

$$
\mathrm{Q}_{\mathrm{R}}=\sum_{\mathrm{i}=1}^{\mathrm{N}_{\mathrm{t}}} \mathrm{Q}_{\mathrm{R} 0_{\mathrm{i}}}\left(\frac{\mathrm{t}-\tau_{0_{\mathrm{i}}}}{\tau_{\mathrm{i}}}\right)^{-\left(\frac{\mathrm{t}-\tau_{0_{i}}}{\tau_{\mathrm{i}}}\right)}
$$

where $\mathrm{N}_{\mathrm{r}}$ is the total number of releases in the GRE. The total release volume is given by the analog to Eq. (2.6) as

$$
\mathrm{V}_{\mathrm{R}}=\frac{1}{\chi_{\mathrm{H}}} \sum_{\mathrm{i}=1}^{\mathrm{N}_{r}} \mathrm{Q}_{\mathrm{R}_{0}} \tau_{\mathrm{i}}
$$

Table 2.1 summarizes this information for the multiple-release analysis for the event in AN-104 that is illustrated in Figure 2.6.

\begin{tabular}{|c|c|c|c|c|c|c|c|}
\hline \multicolumn{4}{|c|}{ Model fit to all data } & \multicolumn{4}{|c|}{ Model fit to individual peaks } \\
\hline \multirow{2}{*}{$\begin{array}{c}\text { Time of } \\
\text { peak }\end{array}$} & \multicolumn{2}{|c|}{$\begin{array}{c}\text { Peak hydrogen } \\
\text { (ppm) }\end{array}$} & \multirow{2}{*}{$\begin{array}{c}\text { Total } \\
\text { hydrogen } \\
\text { release vol } \\
\left(\mathrm{ft}^{\mathbf{3}}\right)\end{array}$} & \multirow[b]{2}{*}{ Time of peak } & \multicolumn{2}{|c|}{$\begin{array}{l}\text { Peak hydrogen } \\
\text { (ppm) }\end{array}$} & \multirow{2}{*}{$\begin{array}{c}\text { Total } \\
\text { hydrogen } \\
\text { release vo } \\
\left(\mathrm{ft}^{\mathbf{3}}\right)\end{array}$} \\
\hline & & odel & & & Data & Model & \\
\hline \multirow{2}{*}{$6 / 2 / 00 \quad 8: 25$} & \multirow{2}{*}{264} & \multirow{2}{*}{277} & \multirow{2}{*}{22} & \begin{tabular}{|l|} 
Initial peak: \\
6/2/00 9:06 \\
\end{tabular} & 266 & 266 & 15 \\
\hline & & & & $\begin{array}{l}\text { Real 2nd peak } \\
\text { 6/2/00 19:48 }\end{array}$ & 107 & 106 & 11 \\
\hline
\end{tabular}

Table 2.1. Comparing Single- and Multiple-Release Analysis Results for AN-104

Treating a GRE as the superposition of a number of individual gas release events makes it possible to apply the gas release model to almost any event, regardless of the shape of the measured concentration history. Figure 2.7 shows additional examples of multiple-release events for GREs with relatively high peak-headspace hydrogen concentrations. None of these appear to consist of a single release, yet all of these are considered BDGREs. If the gas release model is applied by treating each event as a superposition of a number of individual releases, the model estimates the overall gas release behavior quite well. Table 2.2 contains a summary of the total gas release volume for these GREs when analyzed as multiple-release events and compares the results with the values obtained when the model treats the entire event as a single release. 


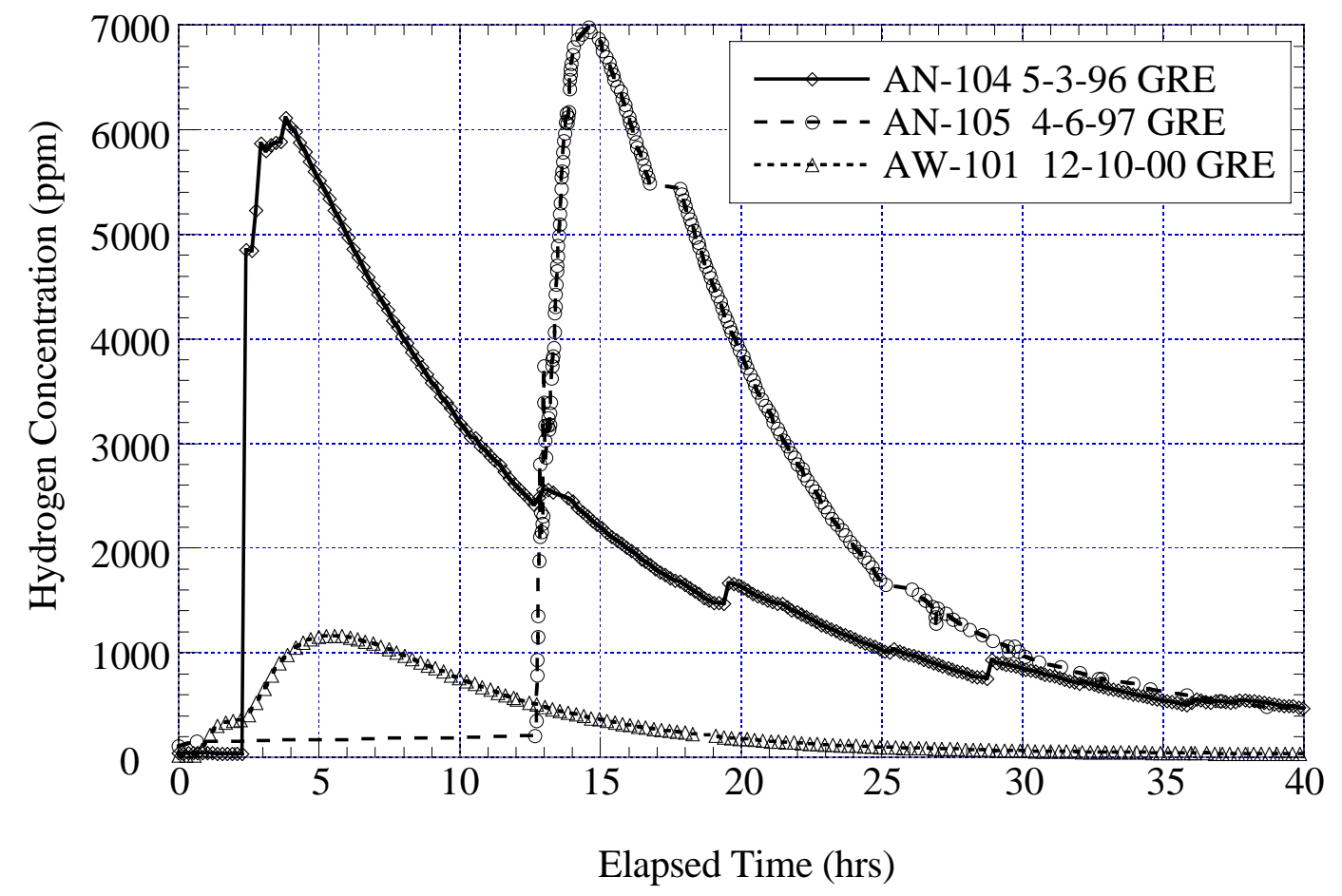

Figure 2.7. Large GREs that Appear to Consist of Multiple Releases

Table 2.2 shows that these two approaches do not produce large differences in the calculated values for total gas release volume obtained for a given event. This is because, even in multiplerelease events, the general shape of a GRE is characterized by a relatively sharp peak in concentration followed by a more or less rapid decay to background concentration levels. For the three relatively large GREs (Figure 2.7), the multiple-release analysis yields total gas release volume about $20-25 \%$ higher than the values obtained by treating the event as a single release. The three GREs illustrated in Figure 2.5a, however, are not as "classical" in shape and are generally of much smaller magnitude overall. Rather than consisting of one large release accompanied by three or four lesser releases, these events appear to consist of a series of releases that are very similar in size. It is thus more difficult to obtain a reasonable estimate of the concentration history when treating the event as a single release, and the difference between the results obtained with the two approaches is greater. For these events, the multiple-release approach is clearly preferable. In any event, the model can be expected to give an accurate estimate of the total release volume only when there is sufficient data to properly characterize the overall shape of the GRE, regardless of the number of actual releases it comprises. This analysis assumes that all GREs are governed by essentially the same physical behavior. Therefore, their hydrogen release behavior is similar.

The time-varying release from a single gob event with a set start time (caused by decant or mixing operations, for example) and total release volume can be modeled using the estimated time to the peak release rate. Table 2.3 summarizes the total gas release volume for GREs in AN-103, AN-104, AN-105, and AW-101 since 1995. This table includes all releases in which 
Table 2.2. Comparing Single- and Multiple-Release Analysis Results for Different GREs

\begin{tabular}{|c|c|c|c|c|c|c|c|}
\hline \multicolumn{4}{|c|}{ Model fit to all data } & \multicolumn{4}{|c|}{ Model fit to individual peaks } \\
\hline \multirow{2}{*}{ Time of peak } & \multicolumn{2}{|c|}{$\left[\mathrm{H}_{2}\right](\mathbf{p p m})$} & \multirow{2}{*}{$\begin{array}{l}\text { Total } \mathbf{H}_{2} \\
\text { rel. vol. } \\
\quad\left(\mathrm{ft}^{\mathbf{3}}\right)\end{array}$} & \multirow{2}{*}{ Time of peak } & \multicolumn{2}{|c|}{$\left[\mathrm{H}_{2}\right](\mathrm{ppm})$} & \multirow{2}{*}{$\begin{array}{c}\text { Total } \mathbf{H}_{2} \\
\text { rel. vol. } \\
\left(\mathbf{f t}^{3}\right)\end{array}$} \\
\hline & Data & Model & & & Data & Model & \\
\hline AN-104 & & & & & & & \\
\hline \multirow{6}{*}{$5 / 4 / 96 \quad 1: 39$} & \multirow{6}{*}{6109} & \multirow{6}{*}{7054} & \multirow{6}{*}{367} & $5 / 4 / 96 \quad 1: 18$ & 6109 & 6144 & 329 \\
\hline & & & & $5 / 4 / 96 \quad 7: 42$ & 447 & 461 & 40 \\
\hline & & & & 5/4/96 12:02 & 378 & 403 & 24 \\
\hline & & & & $5 / 4 / 96 \quad 21: 02$ & 444 & 448 & 42 \\
\hline & & & & $5 / 4 / 965: 41$ & 215 & 223 & 14 \\
\hline & & & & 5/4/96 13:49 & 172 & 170 & 13 \\
\hline & & & & & & & 462 \\
\hline \multirow{4}{*}{ 9/4/99 12:02 } & \multirow{4}{*}{163} & \multirow{4}{*}{163} & \multirow{4}{*}{11} & 9/4/99 12:02 & 152 & 153 & 9 \\
\hline & & & & 9/4/99 20:40 & 32 & 29 & 3 \\
\hline & & & & 9/4/99 22:13 & 18 & 18 & 1 \\
\hline & & & & 9/5/99 19:06 & 12 & 10 & 2 \\
\hline AN-105 & & & & & & & 15 \\
\hline \multirow{5}{*}{$4 / 6 / 97 \quad 23: 46$} & \multirow{5}{*}{6980} & \multirow{5}{*}{7467} & \multirow{5}{*}{347} & 4/6/97 23:46 & 6890 & 7033 & 321 \\
\hline & & & & 4/7/97 3:02 & 988 & 1009 & 43 \\
\hline & & & & 4/7/97 22:14 & 157 & 171 & 20 \\
\hline & & & & 4/8/97 $12: 58$ & 616 & 644 & 33 \\
\hline & & & & 4/8/97 21:24 & 188 & 195 & 20 \\
\hline & & & & & & & 437 \\
\hline \multirow{3}{*}{ 10/28/00 6:09 } & \multirow{3}{*}{205} & \multirow{3}{*}{167} & \multirow{3}{*}{20} & 10/27/00 21:01 & 95 & 76 & 5 \\
\hline & & & & $10 / 28 / 00 \quad 4: 30$ & 142 & 111 & 11 \\
\hline & & & & 10/28/00 7:08 & 51 & 52 & 4 \\
\hline & & & & & & & 19 \\
\hline \multirow{3}{*}{$11 / 27 / 00 \quad 10: 20$} & \multirow{3}{*}{151} & \multirow{3}{*}{149} & \multirow{3}{*}{9} & $11 / 27 / 00 \quad 0: 55$ & 55 & 54 & 3 \\
\hline & & & & $11 / 27 / 00 \quad 7: 50$ & 61 & 62 & 3 \\
\hline & & & & 11/27/00 12:16 & 80 & 78 & 5 \\
\hline AW-101 & & & & & & & 11 \\
\hline & & & & $12 / 10 / 00 \quad 14: 03$ & 353 & 352 & 16 \\
\hline & & & & $12 / 10 / 00 \quad 17: 22$ & 965 & 964 & 64 \\
\hline $12 / 10 / 00 \quad 17: 22$ & 1162 & 1118 & 90 & $12 / 10 / 00 \quad 23: 28$ & 111 & 112 & 10 \\
\hline & & & & $\begin{array}{lll}12 / 10 / 00 & 11: 39\end{array}$ & 29 & 31 & 5 \\
\hline & & & & & & & 95 \\
\hline
\end{tabular}

sufficient data were recorded to adequately characterize the overall shape of the release for the event. (This, in general, means that the data were obtained on at least an hourly interval. For some events, data were obtained only on a daily basis, which may be adequate to determine that a release has occurred but not to ensure catching the peak concentration or to characterize the decay curve. These events were omitted from the analysis results shown in Table 2.3.) 
Table 2.3. Summary of Gas Release Calculations for GREs in AN-103, AN-104, AN-105, and AW-101

\begin{tabular}{|c|c|c|c|c|c|c|c|}
\hline Tank ID & Event ID & Time of Peak & $\begin{array}{c}\text { Measured Peak } \\
\text { Hydrogen (ppm) }\end{array}$ & $\begin{array}{c}\text { Interpolated Peak } \\
\text { Hydrogen (ppm) }\end{array}$ & $\begin{array}{c}\text { Hydrogen Release } \\
\text { Volume }\left(\mathbf{f t}^{\mathbf{3}}\right)\end{array}$ & Tau & $\begin{array}{c}\text { Total Gas } \\
\text { Release }\left(\mathbf{m}^{3}\right)\end{array}$ \\
\hline $\mathrm{AN}-103$ & 8-22-95 GRE & $8 / 22 / 9522: 30$ & 1600 & & 92.407 & 9.406 & 4.29 \\
\hline AN-103 & 2-1-99 GRE & $1 / 30 / 9913: 58$ & 210 & & 13.441 & 0.538 & 0.62 \\
\hline $\mathrm{AN}-103$ & & 2/1/99 10:29 & 560 & & 44.895 & 4.908 & 2.08 \\
\hline AN-103 & 9-4-99 GRE & 9/4/99 8:37 & 1080 & & 71.160 & 9.488 & 3.30 \\
\hline $\mathrm{AN}-103$ & & 9/4/99 11:29 & & 192 & 12.015 & 4.972 & 0.56 \\
\hline $\mathrm{AN}-103$ & & 9/5/99 3:16 & & 149 & 9.677 & 4.696 & 0.45 \\
\hline $\mathrm{AN}-103$ & & 9/5/99 18:11 & & 73 & 4.904 & 0.526 & 0.23 \\
\hline $\mathrm{AN}-103$ & 12-29-99 GRE & 12/30/99 3:27 & 580 & & 33.834 & 40.374 & 1.57 \\
\hline AN-103 & & $12 / 30 / 998: 38$ & & 105 & 7.159 & 21.548 & 0.33 \\
\hline AN-103 & 8-6-00 GRE & 8/6/00 1:56 & 200 & & 10.382 & 11.536 & 0.48 \\
\hline AN-103 & & 8/6/00 9:32 & 210 & & 14.664 & 13.704 & 0.68 \\
\hline $\mathrm{AN}-103$ & & $8 / 7 / 000: 00$ & 115 & & 9.144 & 13.506 & 0.42 \\
\hline AN-103 & & 8/7/00 10:13 & 150 & & 13.475 & 85.335 & 0.63 \\
\hline $\mathrm{AN}-103$ & & 8/8/00 12:38 & 120 & & 10.778 & 132.687 & 0.50 \\
\hline AN-103 & 10-3-00 GRE & 10/3/00 1:54 & 260 & & 11.640 & 19.590 & 0.54 \\
\hline AN-103 & & 10/3/00 21:26 & & 38 & 3.781 & 19.587 & 0.18 \\
\hline AN-103 & 10-20-00 GRE & $10 / 20 / 0017: 14$ & 340 & & 24.022 & 14.130 & 1.12 \\
\hline AN-103 & & $10 / 22 / 006: 48$ & & 189 & 75.986 & 1335.856 & 3.53 \\
\hline AN-104 & 11-6-94 GRE & 11/6/94 17:24 & 3050 & & 178.640 & 45.292 & 11.497 \\
\hline AN-104 & 2-16-95 GRE & 2/16/95 5:08 & 2089 & & 114.592 & 30.548 & 7.375 \\
\hline AN-104 & 10-2-95 GRE & $10 / 2 / 9523: 56$ & 3068 & & 182.271 & 28.728 & 11.730 \\
\hline AN-104 & 10-8-95 GRE & 10/8/95 11:10 & 1800 & & 79.993 & 5.149 & 5.148 \\
\hline AN-104 & 5-3-96 GRE & 5/4/96 1:39 & 6109 & & 329.000 & 14.000 & 21.173 \\
\hline AN-104 & & 5/4/96 7:42 & & 461 & 39.600 & 120.000 & 2.549 \\
\hline AN-104 & & 5/4/96 12:02 & & 403 & 24.000 & 30.000 & 1.545 \\
\hline AN-104 & & 5/4/96 21:02 & & 448 & 42.000 & 150.000 & 2.703 \\
\hline AN-104 & & 5/5/96 5:41 & & 223 & 14.400 & 45.000 & 0.927 \\
\hline AN-104 & & 5/5/96 13:49 & & 170 & 13.287 & 92.395 & 0.855 \\
\hline AN-104 & 5-1-97 GRE & 5/2/97 7:23 & 2250 & & 75.259 & 12.000 & 4.843 \\
\hline AN-104 & & 5/2/97 7:23 & & 800 & 42.732 & 11.006 & 2.750 \\
\hline AN-104 & & 5/2/97 10:49 & & 40 & 1.940 & 1.481 & 0.125 \\
\hline AN-104 & 12-2-98 GRE & 12/2/98 2:04 & 550 & & 27.709 & 22.282 & 1.783 \\
\hline AN-104 & & 12/2/98 4:08 & & 33 & 1.769 & 3.014 & 0.114 \\
\hline
\end{tabular}


Table 2.3. Summary of Gas Release Calculations for GREs in AN-103, AN-104, AN-105, and AW-101

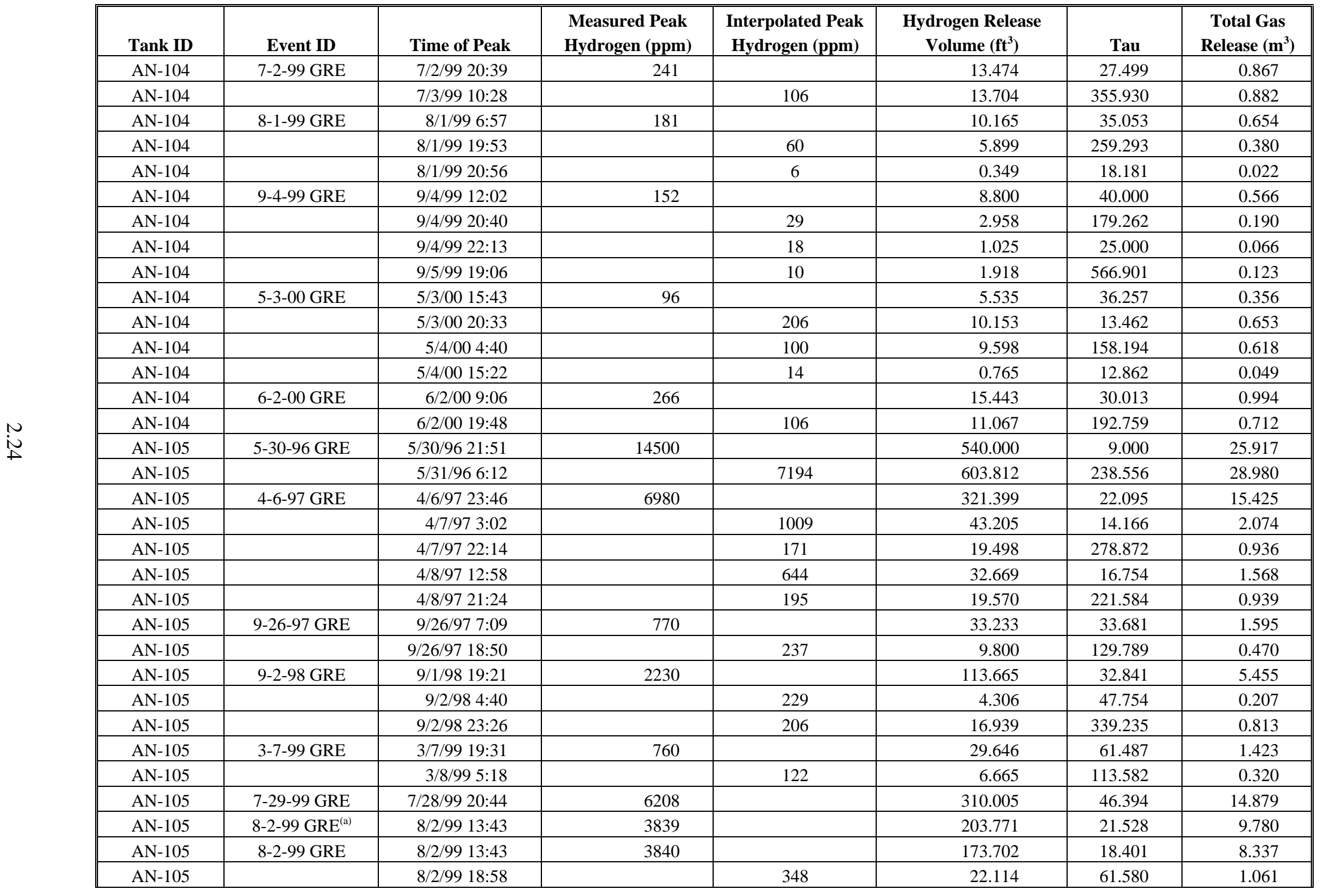


Table 2.3. Summary of Gas Release Calculations for GREs in AN-103, AN-104, AN-105, and AW-101

\begin{tabular}{|c|c|c|c|c|c|c|c|c|}
\hline \multirow{34}{*}{$\stackrel{N}{N}$} & Tank ID & Event ID & Time of Peak & $\begin{array}{c}\text { Measured Peak } \\
\text { Hydrogen (ppm) }\end{array}$ & $\begin{array}{c}\text { Interpolated Peak } \\
\text { Hydrogen (ppm) }\end{array}$ & $\begin{array}{c}\text { Hydrogen Release } \\
\text { Volume }\left(\mathbf{f t}^{\mathbf{3}}\right)\end{array}$ & Tau & $\begin{array}{c}\text { Total Gas } \\
\text { Release }\left(\mathbf{m}^{3}\right) \\
\end{array}$ \\
\hline & AN-105 & & 8/3/99 2:10 & & 102 & 5.507 & 45.888 & 0.264 \\
\hline & $\mathrm{AN}-105$ & & 8/3/99 8:24 & & 28 & 1.987 & 104.677 & 0.095 \\
\hline & AN-105 & & 8/3/99 21:58 & & 42 & 4.519 & 246.967 & 0.217 \\
\hline & AN-105 & 10-27-99 GRE & 10/27/99 23:06 & 180 & & 14.245 & 185.000 & 0.684 \\
\hline & $\mathrm{AN}-105$ & & 10/28/99 4:05 & & 15 & 0.580 & 55.757 & 0.028 \\
\hline & AN-105 & & $10 / 28 / 995: 12$ & & 23 & 0.819 & 34.158 & 0.039 \\
\hline & AN-105 & & 10/29/99 0:02 & & 21 & 1.047 & 83.036 & 0.050 \\
\hline & AN-105 & 12-31-99 GRE & 1/1/00 9:58 & 186 & & 14.217 & 170.383 & 0.682 \\
\hline & $\mathrm{AN}-105$ & 7-21-00 GRE & 7/21/00 22:39 & 146 & & 11.218 & 273.484 & 0.538 \\
\hline & AN-105 & 10-28-00 GRE & 10/27/00 21:01 & & 76 & 5.005 & 182.371 & 0.240 \\
\hline & AN-105 & & 10/28/00 4:30 & & 111 & 10.815 & 210.000 & 0.519 \\
\hline & $\mathrm{AN}-105$ & & $10 / 28 / 007: 08$ & & 52 & 3.636 & 103.872 & 0.174 \\
\hline & AN-105 & 11-27-00 GRE & $11 / 27 / 000: 55$ & 55 & & 3.060 & 180.000 & 0.147 \\
\hline & AN-105 & & $11 / 27 / 007: 50$ & & 62 & 3.300 & 44.000 & 0.158 \\
\hline & $\mathrm{AN}-105$ & & $11 / 27 / 0012: 16$ & & 78 & 4.875 & 75.000 & 0.234 \\
\hline & AN-105 & 12-14-00 GRE & 12/14/00 19:47 & 164 & & 9.016 & 78.530 & 0.433 \\
\hline & AN-105 & & 12/15/00 2:01 & & 25 & 1.677 & 89.006 & 0.080 \\
\hline & $\mathrm{AN}-105$ & & 12/15/00 12:49 & & 9 & 0.538 & 89.006 & 0.026 \\
\hline & $\mathrm{AN}-105$ & 2-23-01 GRE & 2/23/01 18:23 & 82 & & 4.816 & 189.985 & 0.231 \\
\hline & AN-105 & 3-21-01 GRE & $3 / 20 / 0123: 49$ & 337 & & 15.791 & 43.981 & 0.758 \\
\hline & AN-105 & & $3 / 21 / 017: 51$ & & 760 & 43.657 & 72.096 & 2.095 \\
\hline & $\mathrm{AN}-105$ & & 3/21/01 16:09 & & 172 & 6.780 & 38.778 & 0.325 \\
\hline & AN-105 & & $3 / 21 / 0121: 58$ & & 165 & 5.934 & 37.647 & 0.285 \\
\hline & AN-105 & & $3 / 22 / 0114: 10$ & 589 & & 33.735 & 89.087 & 1.619 \\
\hline & $\mathrm{AN}-105$ & & $3 / 23 / 017: 12$ & & 96 & 3.829 & 153.781 & 0.184 \\
\hline & AN-105 & & $3 / 24 / 01$ 19:45 & 166 & & 8.976 & 144.034 & 0.431 \\
\hline & AW-101 & 10-3-94 GRE & 10/3/94 2:00 & 5500 & & 262.043 & 141.158 & 23.188 \\
\hline & AW-101 & 10-5-94 GRE & 10/5/94 11:00 & 6200 & & 211.260 & 75.247 & 18.694 \\
\hline & AW-101 & 11-29-94 GE & 11/28/94 16:00 & 4900 & & 236.716 & 191.089 & 20.947 \\
\hline & AW-101 & 2-23-95 GRE & 2/23/95 10:37 & 4600 & & 193.841 & 42.046 & 17.153 \\
\hline & AW-101 & 5-8-95 GRE & 5/8/95 18:00 & 1800 & & 166.214 & 438.529 & 14.708 \\
\hline & AW-101 & 9-17-95 GRE & 9/17/95 0:00 & 1930 & & 88.618 & 343.684 & 7.842 \\
\hline & AW-101 & 9-23-95 GRE & 9/23/95 8:00 & 1460 & & 45.752 & 51.504 & 4.049 \\
\hline
\end{tabular}


Table 2.3. Summary of Gas Release Calculations for GREs in AN-103, AN-104, AN-105, and AW-101

\begin{tabular}{|c|c|c|c|c|c|c|c|}
\hline Tank ID & Event ID & Time of Peak & $\begin{array}{c}\text { Measured Peak } \\
\text { Hydrogen (ppm) }\end{array}$ & $\begin{array}{c}\text { Interpolated Peak } \\
\text { Hydrogen (ppm) }\end{array}$ & $\begin{array}{c}\text { Hydrogen Release } \\
\text { Volume }\left(\mathbf{f t}^{\mathbf{3}}\right)\end{array}$ & Tau & $\begin{array}{c}\text { Total Gas } \\
\text { Release }\left(\mathbf{m}^{3}\right) \\
\end{array}$ \\
\hline AW-101 & 9-24-95 GRE & 9/25/95 0:00 & 4660 & & 223.040 & 131.954 & 19.737 \\
\hline AW-101 & 10-16-95 GRE & 10/16/95 11:00 & 1750 & & 77.292 & 146.388 & 6.840 \\
\hline AW-101 & 12-29-95 GRE & 12/29/95 17:30 & 6000 & & 251.794 & 66.905 & 22.281 \\
\hline AW-101 & 5-14-96 GRE & 5/14/96 17:56 & 1383 & & 164.934 & 256.634 & 14.595 \\
\hline AW-101 & 10-30-00 GRE & 10/30/00 19:08 & 259 & & 23.489 & 179.997 & 2.079 \\
\hline AW-101 & 1-16-00 GRE & $1 / 14 / 0014: 23$ & & 82 & 7.779 & 457.604 & 0.688 \\
\hline AW-101 & & 1/16/00 9:59 & & 10 & 0.536 & 34.725 & 0.047 \\
\hline AW-101 & & 1/16/00 11:06 & 205 & 88 & 17.109 & 476.253 & 1.514 \\
\hline AW-101 & & $1 / 16 / 0014: 25$ & & 64 & 56.385 & 2569.740 & 4.990 \\
\hline AW-101 & & 1/19/00 21:03 & & 9 & 2.065 & 590.000 & 0.183 \\
\hline AW-101 & & $1 / 19 / 0021: 37$ & & 27 & 30.125 & 3292.183 & 2.666 \\
\hline AW-101 & & 1/20/00 2:19 & & 11 & 0.672 & 48.000 & 0.059 \\
\hline AW-101 & 4-22-00 GRE & $4 / 22 / 004: 35$ & & 2022 & 122.550 & 57.000 & 10.844 \\
\hline AW-101 & & $4 / 22 / 006: 15$ & & 270 & 13.000 & 20.000 & 1.150 \\
\hline AW-101 & & $4 / 22 / 008: 53$ & & 366 & 17.600 & 20.000 & 1.557 \\
\hline AW-101 & & $4 / 22 / 0012: 45$ & & 169 & 11.815 & 80.156 & 1.046 \\
\hline AW-101 & 9-17-00 GRE & 9/17/00 12:08 & 228 & & 30.123 & 436.038 & 2.666 \\
\hline AW-101 & 9-19-00 GRE & $9 / 19 / 0012: 35$ & 212 & & 13.540 & 165.861 & 1.198 \\
\hline AW-101 & 9-22-00 GRE & 9/22/00 3:27 & 356 & & 33.411 & 103.439 & 2.957 \\
\hline AW-101 & & $9 / 22 / 0013: 58$ & & 283 & 13.085 & 9.669 & 1.158 \\
\hline AW-101 & & $9 / 22 / 0017: 51$ & & 403 & 18.200 & 14.000 & 1.611 \\
\hline AW-101 & & 9/23/00 1:44 & & 108 & 11.100 & 185.000 & 0.982 \\
\hline AW-101 & & 9/23/00 17:06 & & 32 & 4.352 & 297.412 & 0.385 \\
\hline AW-101 & 11-19-00 GRE & $11 / 19 / 0010: 32$ & 567 & & 43.965 & 123.000 & 3.890 \\
\hline AW-101 & & 11/19/00 20:47 & & 54 & 6.729 & 256.070 & 0.595 \\
\hline AW-101 & 12-10-00 GRE & 12/10/00 14:03 & 353 & & 16.287 & 21.348 & 1.441 \\
\hline AW-101 & & $12 / 10 / 0017: 22$ & & 964 & 63.914 & 70.000 & 5.656 \\
\hline AW-101 & & $12 / 10 / 0023: 28$ & & 112 & 10.150 & 145.000 & 0.898 \\
\hline AW-101 & & \begin{tabular}{l|l}
$12 / 11 / 00 ~ 11: 39$ \\
\end{tabular} & & 31 & 4.648 & 333.651 & 0.411 \\
\hline \multicolumn{8}{|c|}{ (a) GRE in AN-105 on 8-2-99, treated as a single release. } \\
\hline
\end{tabular}




\subsection{Analysis of Gas Releases Induced by Supernate Decant}

Transfer, or decant, of supernatant liquid has been proposed as a method for retrieval of radioactive waste to the vitrification plant. The supernate decant process is expected to induce BDGREs by reducing the hydrostatic pressure on the gas stored in the sediment layer. A detailed model has been developed that simulates this process. The model is described in Section 3.1, and the parameters used in the model are defined in Section 3.2. Analysis results for Tank AN-105 are presented in Section 3.3, and those for AN-103, AN-104, and AW-101 in Section 3.4. The overall conclusions from the decant analysis are given in Section 3.5.

\subsection{Mathematical Model}

The data and observations pertaining to BDGREs indicate that the sediment layer in those tanks experiencing BDGREs consists of 10 to 15 discrete regions, or "gobs," in different stages of gas retention (Meyer et al. 1997; Meyer and Stewart 2001). In-tank video during large gas releases in SY-101 clearly shows a series of local upwellings from different areas of the tank. Waste temperature profiles before and after GREs observed in other DSTs show the effects of very few of them, indicating localized events. Additionally, based on actual gas release volumes, sediment layer depth, and gas volumes required for buoyancy, only a portion of the sediment layer participates in individual GREs. Finally, this gob concept describes the observed GRE period in relation to the gas generation rate for the BDGRE tanks.

The model simulates the behavior of a number of gobs of gas-bearing sediment during the decant process. It is therefore intended for analyzing tanks that currently exhibit BDGREs. To reiterate, a BDGRE occurs when a portion or gob of the sediment layer accumulates sufficient gas to become buoyant with respect to the overlying liquid. The gob then breaks free of the surrounding sediment and rises to the waste surface, where it releases a portion of its gas into the tank headspace. The gob is assumed to sink back to the sediment layer after releasing enough gas to become negatively buoyant.

This behavior is assumed to occur similarly during decant. The decreasing supernate depth during decant reduces hydrostatic pressure on the gas stored in the sediment and causes it to expand, increasing the gas volume fraction. The initial conditions are determined by tank historical behavior and measured data, as is the actual gas release behavior when a BDGRE occurs during decant. However, gas expansion and approach to buoyancy during decant are not equivalent to accelerated "natural" gas generation and are not modeled as such. Details of the model are presented in this section.

\subsubsection{Depressurization Model}

As discussed, the sediment layer consists of a number of regions or gobs in varying stages of gas retention. Gas release is induced from a gob during decant by the decreasing supernate depth. This reduces hydrostatic pressure on the gas stored in the sediment layer (or individual gobs) and causes it to expand, increasing the gas volume fraction. The assumed waste geometry 
is illustrated in Figure 3.1. The pressure of the gas during the decanting process at a given depth within the sediment layer is given by

$$
\mathrm{P}_{\mathrm{Si}}(\eta)=\mathrm{P}_{\mathrm{A}}+\rho_{\mathrm{L}_{\mathrm{i}-1}} \mathrm{~g}\left(\mathrm{~h}_{\mathrm{C}}+\mathrm{h}_{\mathrm{L}_{\mathrm{i}}}+\eta \mathrm{h}_{\mathrm{S}}\right)
$$

where the subscript $\mathrm{i}$ denotes the time step, $\rho_{\mathrm{L}}$ is the liquid density, $\mathrm{P}_{\mathrm{A}}$ is the atmospheric pressure, and $\mathrm{g}$ is the acceleration due to gravity. The thickness of the crust, supernatant, and sediment layers are denoted by $h_{C}, h_{L}$, and $h_{S}$, respectively. The nondimensional depth $\eta$ is defined as the depth in the sediment layer, $\mathrm{z}$, divided by the sediment layer thickness, $\mathrm{h}_{\mathrm{s}}$. The initial liquid depth is computed from

$$
\mathrm{h}_{\mathrm{L}}=\mathrm{h}_{\mathrm{T}}-\mathrm{h}_{\mathrm{C}}-\mathrm{h}_{\mathrm{S}}
$$

where $\mathrm{h}_{\mathrm{T}}$ is the total waste depth. During a decanting process, the supernatant liquid depth is determined by

$$
\mathrm{h}_{\mathrm{Li}}=\mathrm{h}_{\mathrm{Li}-1}-\mathrm{h}_{\mathrm{R}}-\mathrm{h}_{\mathrm{GASi}-1}
$$

where $h_{R}$ is the depth of liquid removed by the decant during a time step (depends on the decant rate), and $\mathrm{h}_{\mathrm{GAS}}$ is the height associated with the in situ volume of gas released at the previous time step defined by

$$
\mathrm{h}_{\mathrm{GASi}-1}=\frac{\mathrm{P}_{\mathrm{A}}}{\mathrm{P}_{\mathrm{Si}-1}} \frac{\mathrm{Q}_{\mathrm{GASi}-1} \Delta \mathrm{t}}{\mathrm{A}_{\mathrm{T}}}
$$

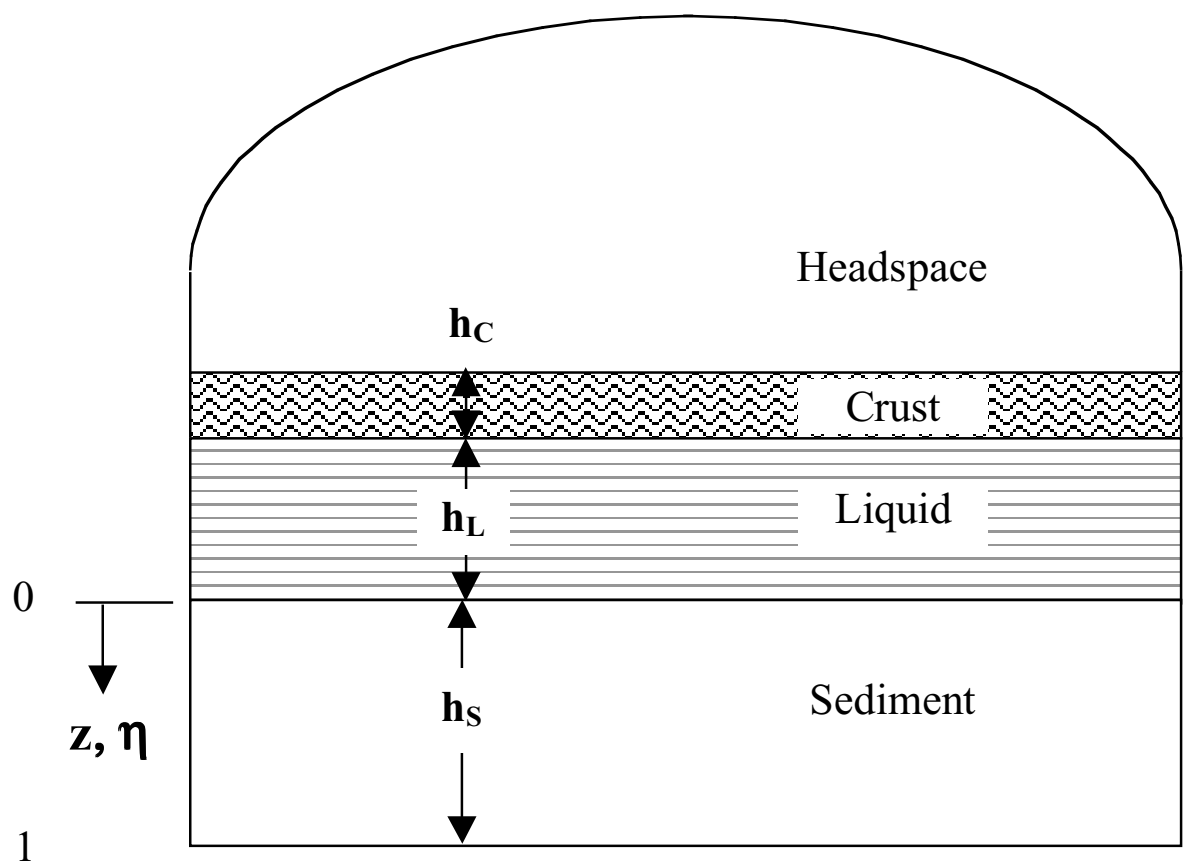

Figure 3.1. Waste Geometry Schematic 
In Eq. (3.4), $\mathrm{Q}_{\mathrm{GASi}-1}$ is the total gas release rate (at headspace pressure) during the previous time step, $\Delta \mathrm{t}$ is the time step, and $\mathrm{A}_{\mathrm{T}}$ is the tank cross-sectional area. This term accounts for the "replacement" of gas released from the sediment layer by supernatant liquid. In addition to the reduction of the supernatant liquid thickness, the average pressure of the gas is also altered by any change in the liquid density due to suspension of released gob material, as is discussed below.

The gas volume fraction or "void fraction" in a gob is defined as

$$
\alpha=\frac{\mathrm{V}_{\mathrm{G}}}{\mathrm{V}_{\mathrm{G}}+\mathrm{V}_{\mathrm{NG}}}
$$

where $V_{G}$ is the gas volume and $V_{N G}$ is the nongas volume of solids and interstitial liquid. The effect of the pressure change on the gas volume fraction in the gob(s) can be determined from the ideal gas law to be

$$
\frac{\mathrm{d} \alpha}{\mathrm{dP}_{\mathrm{S}}}=-\frac{\alpha(1-\alpha)}{\mathrm{P}_{\mathrm{S}}}
$$

Measured gas fraction profiles appear to approximate either a linear or parabolic vertical profile over the depth of the sediment layer. Both profiles may be present in a given tank (Hedengren et al. 2000). We postulate that these profiles develop in the following manner. Gas generation and retention in an initially gasless sediment layer will develop a parabolic vertical gas fraction profile (Meyer and Stewart 2001). When a gob with a parabolic profile goes buoyant, only the portion that is buoyant participates. This leaves a portion of the gob on the tank bottom with whatever gas it has already accumulated. As degassed material refills above this portion and the gob accumulates gas for its next cycle, there is initially more gas on the bottom, and the gob assumes a linear profile. When this gob reaches buoyancy, all of it participates in a BDGRE. A parabolic gas fraction profile will then re-develop, and so on. Allowing for a vertical distribution of gas fraction in a gob, the following expression for the gas fraction can be determined by integrating Eq. (3.6) from the initial conditions (denoted by the subscript 0 ) to time i:

$$
\alpha_{i}(\eta)=\left[\frac{P_{i}(\eta)}{P_{0}(\eta)}\left(\frac{1-\alpha_{0}(\eta)}{\alpha_{0}(\eta)}\right)+1\right]^{-1}
$$

The change of the average gas fraction in a gob during a decant process can therefore be determined from

$$
\alpha_{\mathrm{Ai}, \mathrm{n}}=\frac{1}{\eta_{2}-\eta_{1}} \int_{\eta_{1}}^{\eta_{2}} \alpha_{\mathrm{i}, \mathrm{n}}(\eta) \mathrm{d} \eta
$$


where the subscript $n$ denotes the particular gob. The limits, $\eta_{1}$ and $\eta_{2}$, are dependent on the vertical gas fraction profile in the gob. The cumulative effect of gas generation on the retained gas volume over the several days required for decant is negligible and is ignored.

The gas fraction profile $\alpha_{0}(\eta)$ in Eq. (3.7) is defined by either

$$
\alpha_{0}(\eta)=2 \alpha_{\mathrm{A} 0 \mathrm{n}} \eta
$$

for a linear profile gob or

$$
\alpha_{0}(\eta)=\frac{16}{3} \alpha_{A 0 n} \eta(1-\eta)
$$

for a parabolic gas fraction profile, where $\alpha_{\mathrm{A} 0 \mathrm{n}}$ is the average initial gas fraction in the gob. These profiles are depicted in Figure 3.2.

The average gas fraction growth during a decant is determined by substituting Eq. (3.9) and (3.10) into Eq. (3.8). The limits of the definite integral are dependent on the vertical gas fraction profile. For a gob to become buoyant, its average gas fraction must be the neutral buoyant gas fraction defined by

$$
\alpha_{\mathrm{NBi}}=1-\frac{\rho_{\mathrm{Li}-1}}{\rho_{\mathrm{S}}}
$$

where $\rho_{s}$ is the sediment or gob degassed density. For a linear gas fraction profile gob the limits are the top and bottom of the sediment layer, so $\eta_{1}$ (recalling that $\eta$ is the depth into the sediment layer divided by the sediment layer thickness) is zero, and $\eta_{2}$ is equal to unity.

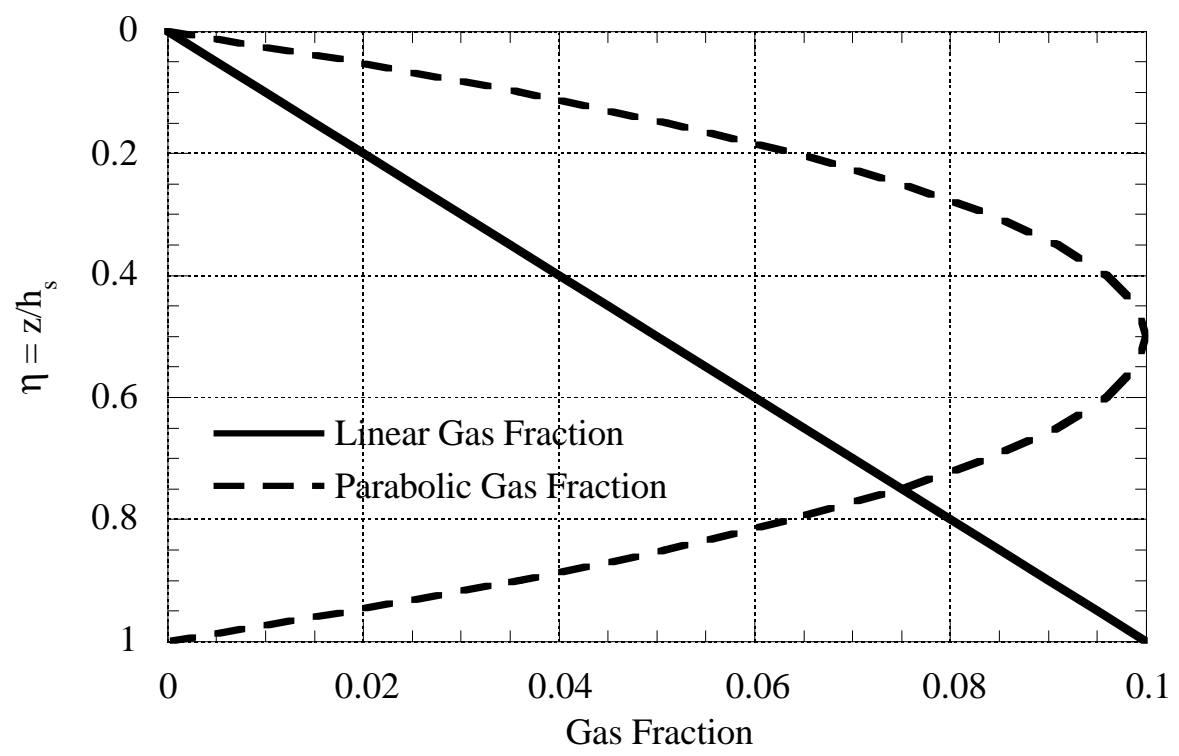

Figure 3.2. Linear and Parabolic Gas Fraction Profiles 
For a gob with a parabolic gas fraction profile Eq. (3.10) to become buoyant, its average gas fraction necessarily must also be the neutral buoyant gas fraction. However, the low gas fraction at the bottom of a parabolic profile gob will reach this condition only after the higher gas fraction regions above have already exceeded neutral buoyancy. This is obviously nonphysical. It is clear, therefore, that not all of the gob will reach buoyancy at the same time. The depth in the sediment layer at which that point and the material above it reach buoyancy is the depth at which both the gas fraction at that point and the average gas fraction from the top of the sediment layer down to that point are both equal to the neutral buoyant gas fraction. We define that point as the critical depth into the sediment layer, $\eta_{\mathrm{C}}$, or

$$
\alpha_{A}\left(\eta_{C}\right)=\frac{\frac{16}{3} \alpha_{A 0 n}}{z_{C}} \int_{0}^{\eta_{C}} \eta(1-\eta) d \eta=\frac{16}{3} \alpha_{A 0 n} \eta_{C}\left(\frac{1}{2}-\frac{\eta_{C}}{3}\right)=\alpha_{N B}
$$

At height $\eta_{C}$, the gas fraction must also be equal to neutral buoyancy, $\alpha\left(\eta_{C}\right)=\alpha_{N B}$, so

$$
\frac{16}{3} \alpha_{\mathrm{A} 0 \mathrm{n}} \eta_{\mathrm{C}}\left(\frac{1}{2}-\frac{\eta_{\mathrm{C}}}{3}\right)=\frac{16}{3} \alpha_{\mathrm{A} 0 \mathrm{n}} \eta_{\mathrm{C}}\left(1-\eta_{\mathrm{C}}\right)
$$

Solving Eq. (3.13) for the critical depth $\eta_{\mathrm{C}}$ yields 0.75. The limits of integration on Eq. (3.8) for a parabolic profile gob are then $\eta_{1}=0$ and $\eta_{2}=0.75$. This implies that the average gas fraction of interest in regard to buoyant conditions during a decant in a parabolic gob is that at $\eta=0.75$, or $3 / 4$ of $h_{s}$.

\subsubsection{Buoyancy Threshold and Gas Release/Material Suspension Models}

Gobs with a sufficient initial retained gas volume will become buoyant due to the decant and undergo BDGREs. The gas fraction required for release is slightly greater than for neutral buoyancy to account for the restraining effect of the surrounding nonbuoyant sediment and is defined as the critical gas fraction (Meyer et al. 1997) or

$$
\alpha_{\mathrm{Ci}}=\alpha_{\mathrm{NBi}}+\frac{\beta \tau_{\mathrm{y}}}{\rho_{\mathrm{S}} \mathrm{gh}_{\mathrm{S}}}
$$

where $\beta$ is ratio of the yield stress in tension to the yield stress in pure shear. The stress at yielding may be given by $\beta \tau_{\mathrm{y}}$, where $1 \leq \beta \leq \sqrt{3} . \beta=1$ is used in this study. Therefore, when $\alpha_{\mathrm{A}, \mathrm{n}} \geq \alpha_{\mathrm{C}}$, the gob rises to the waste surface and may release a portion of its gas.

While a BD can occur when the gas volume fraction in a gob reaches the critical value, gas release will be minimal unless the buoyant potential energy exceeds the energy required to yield the displaced material. In addition, a large fraction of the buoyant energy is dissipated in other processes, so the required buoyant energy is greater than that which would just yield the waste. The energy model (Stewart et al. 1996) introduced in Section 1 is used to account for these phenomena. In the decant model, the "energy model" is the ratio between the buoyant energy, 
$\mathrm{E}_{\mathrm{b}}$, and the energy required to yield the gas-bearing gob participating in the buoyant displacement, $\mathrm{E}_{\mathrm{y}}$, given by

$$
\frac{\mathrm{E}_{\mathrm{b}}}{\mathrm{E}_{\mathrm{y}}}=\frac{\alpha_{\mathrm{Ai}, \mathrm{n}} \rho_{\mathrm{Li}-1} \mathrm{gh}_{\mathrm{i}}}{\left(1-\alpha_{\mathrm{Ai}, \mathrm{n}}\right) \varepsilon_{\mathrm{y}} \tau_{\mathrm{y}}}\left(\left(1+\frac{1}{\gamma}\right) \ln (1+\gamma)-\mathrm{k}\right\rfloor
$$

where $\varepsilon_{\mathrm{y}}$ is the strain at failure, which is taken to be unity. The distance (h) through which the participating gob can rise and release potential energy is computed based on the actual buoyancy of the gob at the surface and is defined by

$$
\mathrm{h}_{\mathrm{i}}=\mathrm{h}_{\mathrm{Li}}+\mathrm{h}_{\mathrm{S}}\left\{\frac{1}{2}+\frac{1}{2}\left[\mathrm{P}_{\mathrm{Si}} \alpha_{\mathrm{Ai}, \mathrm{n}}+\left(1-\alpha_{\mathrm{Ai}, \mathrm{n}}\right)\right]^{\frac{1}{3}}-\frac{\rho_{\mathrm{S}}}{\rho_{\mathrm{L}}}\left(1-\alpha_{\mathrm{Ai}, \mathrm{n}}\right)\left[\mathrm{P}_{\mathrm{Si}} \alpha_{\mathrm{Ai}, \mathrm{n}}+\left(1-\alpha_{\mathrm{Ai}, \mathrm{n}}\right)\right]^{-\frac{2}{3}}\right\}
$$

The derivation of Eq. (3.16) is provided in Appendix B. The parameters $\gamma$ and $\mathrm{k}$ are determined from

$$
\gamma=\frac{\rho_{\mathrm{Li}-1} \mathrm{gh}_{\mathrm{i}}}{\mathrm{P}_{\mathrm{A}}}
$$

and

$$
k=\frac{\alpha_{\mathrm{NB}}\left(1-\alpha_{\mathrm{Ai}}\right)}{\alpha_{\mathrm{Ai}}\left(1-\alpha_{\mathrm{NB}}\right)}
$$

Criteria for determining the potential for gas release from a buoyant gob with the energy model (h is given in Eq. 3.16) have been developed by applying the energy model to the results of a set of scaled experiments and to Hanford DSTs that have historically exhibited BDGRE behavior. The input parameters of six DSTs and their energy ratios are summarized in Table 3.1. It is important to note that these inputs are median values and, as well characterized as these DSTs are, uncertainties in their waste properties can produce uncertainties in the model results.

AW-101 has the highest energy ratio and SY-103 the smallest. Because all of the tanks under consideration exhibit periodic BDGREs, the tank results imply that an energy ratio greater than 7.0 indicates that there is sufficient energy in a BD to release a large fraction of its gas.

The results of scaled BD experiments (Stewart et al. 1996) were also evaluated. These experiments used a bentonite clay simulant with gas produced in situ by the decomposition of hydrogen peroxide. The model inputs and results are shown in Table 3.2. In the first test case a displacement occurred, but minimal gas was released because the individual gobs did not break apart and release their gas while rising to the surface. An energy ratio of 4.1 was determined for this case. 
Table 3.1. Select Hanford DST Waste Properties and Energy Ratio Results (Hedengren et al. 2000; Moore et al. 2000; TWINS database)

\begin{tabular}{||l|c|c|c|c|c|c||}
\hline Property/Tank & AN-103 & AN-104 & AN-105 & AW-101 & SY-101 $^{\text {(a) }}$ & SY-103 \\
\hline \multicolumn{7}{|c||}{ Density $\left(\mathrm{kg} / \mathrm{m}^{3}\right)$} \\
\hline Supernatant Layer & 1450 & 1440 & 1430 & 1430 & 1490 & 1470 \\
\hline Sediment Layer & 1737 & 1579 & 1572 & 1553 & 1700 & 1600 \\
\hline \multicolumn{7}{|c|}{ Layer Thickness (m) } \\
\hline Waste Level & 8.84 & 9.72 & 10.41 & 10.40 & 10.54 & 6.91 \\
\hline Crust Layer & 0.89 & 0.41 & 0.45 & 0.80 & 1.00 & 0.58 \\
\hline Supernatant Layer & 4.17 & 5.26 & 5.46 & 6.74 & 3.70 & 3.08 \\
\hline Sediment Layer & 3.78 & 4.05 & 4.50 & 2.86 & 5.84 & 3.25 \\
\hline \multicolumn{7}{|c||}{ Sediment Layer Rheology } \\
\hline Yield Stress (Pa) & 160 & 125 & 135 & 150 & 116 & 150 \\
\hline \multicolumn{7}{|c|}{ Energy Ratio } \\
\hline $\mathrm{E}_{\mathrm{b}} / \mathrm{E}_{\mathrm{y}}$ & \multicolumn{7}{|c|}{21} & 21 & 25 & 21 & 7 \\
\hline (a) SY-101 data represent pre-mixer pump conditions (pre 1993). \\
\hline \hline
\end{tabular}

Table 3.2. Scaled Buoyant Displacement Experiments Material Properties and Energy Ratio Results

\begin{tabular}{|l|c|c|c|c||}
\hline \hline Property/Case & $\mathbf{1}$ & $\mathbf{2}$ & $\mathbf{3}$ & $\mathbf{4}$ \\
\hline \multicolumn{5}{|c||}{ Density $\left(\mathrm{kg} / \mathrm{m}^{3}\right)$} \\
\hline Supernatant Layer & 1000 & 1000 & 1000 & 1000 \\
\hline Sediment Layer & 1087 & 1087 & 1070 & 1070 \\
\hline \multicolumn{5}{|c||}{ Layer Thickness (m) } \\
\hline Supernatant Layer & 0.105 & 0.012 & 0.101 & 0.011 \\
\hline Sediment Layer & 0.047 & 0.045 & 0.048 & 0.048 \\
\hline \multicolumn{5}{|c||}{ Sediment Layer Rheology } \\
\hline Yield Stress (Pa) & 67 & 67 & 14 & 14 \\
\hline \multicolumn{5}{|c||}{ Gas Release Behavior } \\
\hline Energetic & Yes & No & Yes & No \\
\hline Gas Release & Minimal & No & Yes & No \\
\hline \multicolumn{7}{|c||}{ Energy Ratio } \\
\hline $\mathrm{E}_{\mathrm{b}} / \mathrm{E}_{\mathrm{y}}$ & 4.1 & 0.7 & 7.9 & 2.3 \\
\hline \hline
\end{tabular}

The conditions of the second case are the same as those of the first, but the depth of the liquid layer is reduced. For this case, no active displacement or gas release was observed. The model predicts an energy ratio of 0.7 . In the third and fourth cases, a weaker simulant was used. In Case 3, with a predicted energy ratio of 7.9, both an energetic displacement and a gas release were observed. In the final case the liquid layer was reduced, and again no active displacement was observed. A small amount of gas was released, but the mechanism appeared to be percolation rather than buoyant displacement. The energy ratio for this case is 2.3. 
The energy ratio for SY-103 is lower than the energy ratio of Case 3. Case 3, however, compared well visually with the large, very energetic BDs videotaped in SY-101 and is therefore considered to represent a more severe event.

While these observations and the scaled experiment results are not enough to precisely quantify the relation between gas release and energy ratio, they are consistent with the following criteria: no disruptive $\mathrm{BD}$ is predicted for $\mathrm{E}_{\mathrm{b}} / \mathrm{E}_{\mathrm{y}}<1$, BDs with limited gas release might occur for $\mathrm{E}_{\mathrm{b}} / \mathrm{E}_{\mathrm{y}} \geq 4$, and major gas releases can be expected if $\mathrm{E}_{\mathrm{b}} / \mathrm{E}_{\mathrm{y}} \geq 7$. For the decant analysis, gobs that become buoyant with an energy ratio less than 4.0 are assumed not to release gas.

The total gas release volumes from those gobs that have enough energy to release their gas are determined from the gob's conditions and the condition of the supernatant layer at the time the gob becomes buoyant. The volume of gas in a gob that has risen to the waste surface can be determined from

$$
\mathrm{V}_{\text {gasAi,n }}=\frac{\mathrm{P}_{\mathrm{Si}}}{\mathrm{P}_{\mathrm{A}}} \mathrm{V}_{\text {gobn }} \alpha_{\mathrm{Ai}, \mathrm{n}}
$$

where $\mathrm{V}_{\mathrm{gob}}$ is the bulk gob volume. In this analysis, the gobs are assumed to be right-circular cylinders with depths equal to the thickness of the sediment layer. All of the sediment volume is assumed to be composed of these gobs. Although this shape is a construct to allow for computational convenience, it is acceptable given that the exact shape of the gobs is unknown. Note that the previously discussed vertical gas fraction profiles in the sediment layer dictate the depth of a gob that will participate in a BDGRE. Further, the pertinent quantity is the gas volume contained in and released from a gob, which is dependent on total gob volume, not gob shape. The gob volume of a buoyant gob may therefore be expressed as

$$
\mathrm{V}_{\text {gobn }}=\frac{\pi}{4} \mathrm{D}_{\mathrm{n}} \mathrm{h}_{\mathrm{Gn}}
$$

where $D_{n}$ is the gob diameter and $h_{G n}$ is the gob height $\left(h_{S}\right.$ for linear gas fraction profile and $3 / 4$ $\mathrm{h}_{\mathrm{S}}$ for a parabolic gas fraction profile).

The total volume of gas released from the gob is given by

$$
\mathrm{V}_{\text {re ln }}=\mathrm{V}_{\text {gasAi,n }} \mathrm{f}_{\text {reli }}
$$

Assuming that gas is released at the waste surface until neutral buoyant conditions, the fraction of the gas released, or $\mathrm{f}_{\text {rel }}$, is given by

$$
\mathrm{f}_{\text {reli }}=1-\frac{\alpha_{\mathrm{NBi}}\left(1-\alpha_{\mathrm{Ai}}\right)}{\alpha_{\mathrm{Ai}}\left(1-\alpha_{\mathrm{NBi}}\right)} \frac{\mathrm{P}_{\mathrm{A}}}{\mathrm{P}_{\mathrm{Si}}}
$$

Combining Eq. (3.21) and (3.22) yields 


$$
\mathrm{V}_{\text {reln }}=\mathrm{V}_{\text {gobn }}\left[\left(\frac{\mathrm{P}_{\mathrm{Si}}}{\mathrm{P}_{\mathrm{A}}}+\frac{\alpha_{\mathrm{NBi}}}{\left(1-\alpha_{\mathrm{NBi}}\right)}\right) \alpha_{\mathrm{Ai}, \mathrm{n}}-\frac{\alpha_{\mathrm{NBi}}}{\left(1-\alpha_{\mathrm{NBi}}\right)}\right]
$$

When a BDGRE occurs, gas is assumed to be released from the gob at the same time-varying rate as during spontaneous BDGREs. The average gas release rate at time $\mathrm{i}$ from an individual gob can be computed from the release rate model presented in Hedengren et al. (2000):

$$
Q_{R n}=Q_{R 0 n}\left(\frac{t-t_{0 n}}{\tau_{n}}\right)^{-\frac{t-t_{0 n}}{\tau_{n}}}
$$

where $\mathrm{Q}_{\mathrm{R} 0 \mathrm{n}}$ is the peak gas release rate $\left(\mathrm{V}_{\text {reln }} / \tau\right)$ for gob $\mathrm{n}, \mathrm{t}_{0 \mathrm{n}}$ is the gas release start time, and $\tau_{\mathrm{n}}$ is the time to the peak release rate (particular to each gob, see Section 3.2). Because the release rate changes much more rapidly than the headspace hydrogen concentration, it is helpful to integrate Eq. (3.24) to get the average release rate over a time step so the correct release volume will be used even with relatively large time steps. The average gas release rate, $\mathrm{Q}_{\mathrm{Ai}, \mathrm{n}}$, is expressed as

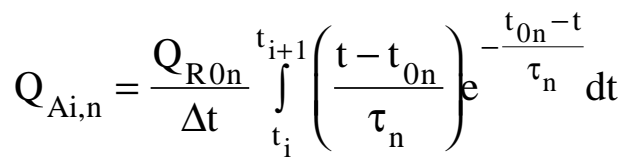

Carrying out the integration gives

$$
\mathrm{Q}_{\mathrm{Ai}, \mathrm{n}}=\frac{\mathrm{V}_{\mathrm{re} \ln }}{\Delta \mathrm{t}}\left\lfloor\left(\frac{\mathrm{t}_{\mathrm{i}}-\mathrm{t}_{0 \mathrm{n}}}{\tau_{\mathrm{n}}}+1\right)^{\frac{\mathrm{t}_{0 \mathrm{n}}-\mathrm{t}_{\mathrm{i}}}{\tau_{\mathrm{n}}}}-\left(\frac{\mathrm{t}_{\mathrm{i}+1}-\mathrm{t}_{0 \mathrm{n}}}{\tau_{\mathrm{n}}}+1\right)^{\frac{\mathrm{t}_{0 n}-\mathrm{t}_{\mathrm{i}+1}}{\tau_{\mathrm{n}}}}\right\rfloor
$$

The total gas release rate at time $\mathrm{i}$ during the decant $\left(\mathrm{Q}_{\mathrm{GAS}}\right.$ in Eq. 3.4) is simply the sum of $\mathrm{Q}_{\mathrm{Ai}, \mathrm{n}}$ over all gobs, from which the concentration of hydrogen in the headspace can be computed (see Section 3.1.3).

The gas release process is assumed to result from a fraction of the gob volume disintegrating or breaking up to allow bubbles to escape. A fraction of the portion of the gob that disintegrates in this process is assumed to remain suspended and become mixed in the supernate and not resettle with the rest of the spent gob. The fractional rate of mixing is assumed to be the same as the fractional gas release rate. The volume of gob material released into the supernate is therefore given by setting the peak gas release rate in Eq. (3.24) to $1 / \tau$ (from $\mathrm{Q}_{\mathrm{R} 0} \tau=1.0$ : i.e., maximum release of solids at the maximum gas release rate). Carrying out the integration and multiplying by the nongaseous volume of the gob gives 


$$
V_{\text {gobRi,n }}=V_{N G n} f_{\text {rel0n }}\left\lfloor\left(\frac{t_{i}-t_{0 n}}{\tau_{n}}+1\right) e^{\frac{t_{0 n}-t_{i}}{\tau_{n}}}-\left(\frac{t_{i+1}-t_{0 n}}{\tau_{n}}+1\right) e^{\frac{t_{0 n}-t_{i+1}}{\tau_{n}}}\right\rfloor
$$

where $f_{\text {rel0n }}$ is the fraction of the gas released at the start time of the gas release, and $V_{\mathrm{NGn}}$ is the nongaseous volume of the gob given by

$$
\mathrm{V}_{\mathrm{NGn}}=\mathrm{V}_{\mathrm{gobn}}\left(1-\alpha_{\mathrm{Ai}, \mathrm{n}}\right)
$$

with the gas fraction at the time of release. The volume of gob material suspended in the supernate is given by

$$
\mathrm{V}_{\text {gobSi }}={ }^{\text {gob }} \sum_{\mathrm{n}=1}^{\text {count }} \mathrm{V}_{\text {gobRi, } \mathrm{n}} \mathrm{F}
$$

where $\mathrm{F}$, the fraction of this released material that remains suspended in the supernate, is determined from historical release data, as discussed in Section 3.2. The gob count, or the number of gobs in the tank, is also addressed in Section 3.2.

Material thus mixing in the supernate is assumed to remain there for the duration of the simulation. Suspension of the heavier solid particles increases the supernate density and reduces the neutral buoyancy gas fraction for the gobs that have not previously become buoyant. This may make additional gobs buoyant immediately and allow subsequent gobs to become buoyant earlier than they would have without mixing. The dominant effect is to allow multiple BDGREs to be triggered by preceding events. This phenomenon is postulated to cause the multiple release behavior observed in the tanks, as discussed in Section 2.

The liquid density $\left(\rho_{L}\right)$ at time $i$ accounts for the suspension of gob material and is given by

$$
\rho_{\mathrm{Li}}=\frac{\left(\mathrm{h}_{\mathrm{Li}} \mathrm{A}_{\mathrm{T}}+\sum_{\mathrm{t}=1}^{\mathrm{i}-1} \mathrm{v}_{\mathrm{gobSt}}\right) \rho_{\mathrm{Li}-1}+\mathrm{V}_{\mathrm{gobSi}} \cdot \rho_{\mathrm{S}}}{\mathrm{h}_{\mathrm{Li}} \mathrm{A}_{\mathrm{T}}+\sum_{\mathrm{t}=1}^{\mathrm{i}-1} \mathrm{~V}_{\mathrm{gobSt}}+\mathrm{V}_{\text {gobSi }}}
$$

\subsubsection{Headspace Hydrogen Concentration Model}

The headspace hydrogen concentration is computed from $\mathrm{Q}_{\mathrm{GASi}} \Delta \mathrm{t}$ and the background gas generation of the remaining waste (supernate and sediment). Consider a tank containing variable volumes of waste and headspace with inflows and outflows as indicated in Figure 3.3. The ventilation inflow, $\mathrm{Q}_{\mathrm{VIN}}$, and outflow, $\mathrm{Q}_{\mathrm{VOUT}}$, carry air into and the headspace atmosphere out of the tank headspace volume, $\mathrm{V}_{\mathrm{HS}}$, respectively. During decant, liquid is removed from the convective layer at the rate $\mathrm{Q}_{\mathrm{LOUT}}$. Gas is released from the waste to the headspace at the 


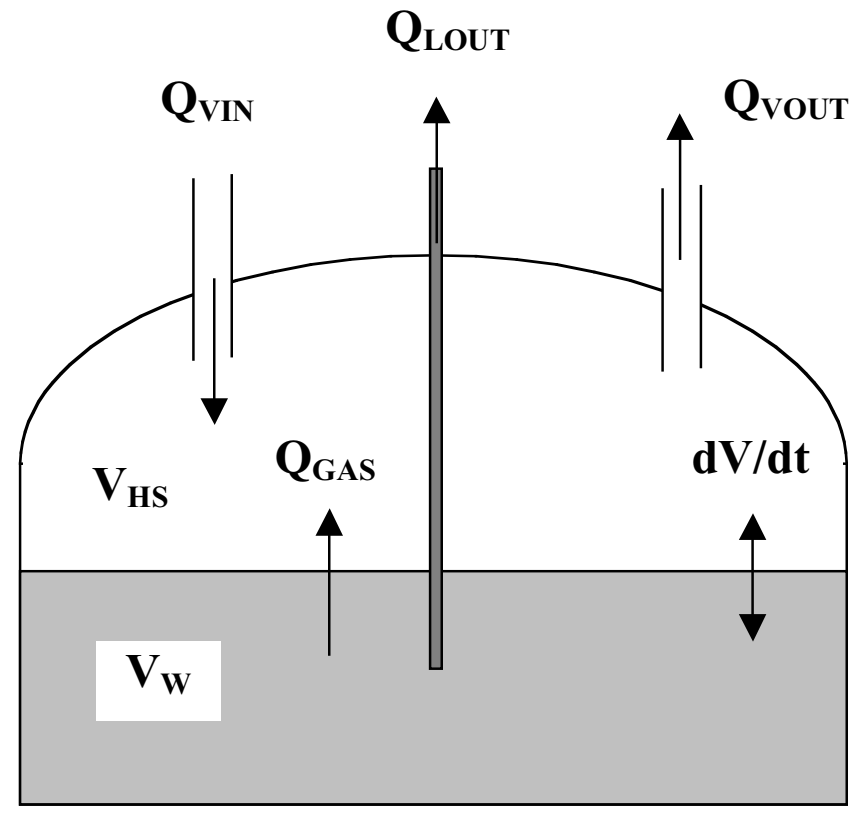

Figure 3.3. Schematic of Tank Flow Paths

volumetric rate $\mathrm{Q}_{\mathrm{GAS}}$. The headspace atmosphere and the liquid/gas/solid mixture in the waste volume are both assumed to be incompressible for the headspace hydrogen concentration analysis. We define $\mathrm{Q}_{\mathrm{LOUT}}$ and $\mathrm{Q}_{\mathrm{VOUT}}$ as positive for outflow, $\mathrm{Q}_{\mathrm{VIN}}$ as positive for inflow, and $\mathrm{Q}_{\mathrm{GAS}}$ as positive for flow from the waste into the headspace.

Let the volume of the tank be fixed, but allow the headspace volume and waste volume to vary. The incompressible fluid assumption requires that

$$
\frac{\mathrm{dV}_{\mathrm{HS}}}{\mathrm{dt}}=-\frac{\mathrm{dV}_{\mathrm{W}}}{\mathrm{dt}}
$$

The headspace volume during the decant is computed from

$$
\mathrm{V}_{\mathrm{HSi}}=\mathrm{V}_{\mathrm{T}}-\left(\mathrm{h}_{\mathrm{C}}+\mathrm{h}_{\mathrm{Li}}+\mathrm{h}_{\mathrm{S}}\right) \mathrm{A}_{\mathrm{T}}
$$

where $\mathrm{V}_{\mathrm{T}}$ is the tank volume. Continuity on the headspace volume can be expressed as

$$
\frac{\mathrm{dV}_{\mathrm{HS}}}{\mathrm{dt}}=\mathrm{Q}_{\mathrm{VIN}}-\mathrm{Q}_{\mathrm{VOUT}}+\mathrm{Q}_{\mathrm{GAS}}
$$

assuming the released gas leaves the waste at the pressure that retained it, $\mathrm{P}_{\mathrm{GAS}}$, and expands isothermally to the headspace pressure, $\mathrm{P}_{\mathrm{HS}}$. Also assuming that the effect of retained gas expansion in the waste due to decrease in hydrostatic pressure is minimal, continuity on the waste volume is written as 


$$
\frac{\mathrm{dV}_{\mathrm{W}}}{\mathrm{dt}}=-\mathrm{Q}_{\text {LOUT }}-\frac{\mathrm{P}_{\mathrm{HS}}}{\mathrm{P}_{\mathrm{GAS}}} \mathrm{Q}_{\mathrm{GAS}}
$$

Substituting Eq. (3.33) and (3.34) into Eq. (3.31) provides an expression for the ventilation outflow, $Q_{\text {out }}$, as a function of the other known inflows and outflows:

$$
\mathrm{Q}_{\mathrm{VOUT}}=\mathrm{Q}_{\mathrm{VIN}}+\mathrm{Q}_{\mathrm{GAS}}\left(1-\frac{\mathrm{P}_{\mathrm{HS}}}{\mathrm{P}_{\mathrm{GAS}}}\right)-\mathrm{Q}_{\mathrm{LOUT}}
$$

The continuity equation for hydrogen in the headspace, assuming that the hydrogen concentration remains small, can be written as

$$
\frac{\mathrm{d}}{\mathrm{dt}}\left(\mathrm{C}_{\mathrm{H}} \mathrm{V}_{\mathrm{HS}}\right)=\mathrm{Q}_{\mathrm{GAS}} \chi_{\mathrm{H}}-\mathrm{Q}_{\mathrm{VOUT}} \mathrm{C}_{\mathrm{H}}
$$

where $\mathrm{C}_{\mathrm{H}}$ is the headspace hydrogen concentration ( $\mathrm{vol} \%$ ) and $\chi_{\mathrm{H}}$ is the volume fraction of hydrogen in the waste gas. Expanding the derivative and substituting Eq. (3.34) and (3.35) via Eq. (3.31) yields

$$
\frac{\mathrm{dC}_{\mathrm{H}}}{\mathrm{dt}}=\frac{1}{\mathrm{~V}_{\mathrm{HS}}}\left(\mathrm{Q}_{\mathrm{GAS}} \chi_{\mathrm{H}}-\left(\mathrm{Q}_{\mathrm{VIN}}-\mathrm{Q}_{\mathrm{GAS}}\right) \mathrm{C}_{\mathrm{H}}\right)
$$

An implicit finite difference analog to Eq. (3.37) for time step $\Delta t$ is

$$
\frac{\mathrm{C}_{\mathrm{Hi}}-\mathrm{C}_{\mathrm{Hi}-1}}{\Delta \mathrm{t}}=\frac{\mathrm{Q}_{\mathrm{GASi}} \cdot \chi_{\mathrm{H}}-\mathrm{C}_{\mathrm{Hi}}\left(\mathrm{Q}_{\mathrm{VIN}}+\mathrm{Q}_{\mathrm{GASi}}\right)}{\mathrm{V}_{\mathrm{HSi}}}
$$

Solving Eq. (3.38) for $\mathrm{C}_{\mathrm{Hi}}$ gives

$$
\mathrm{C}_{\mathrm{Hi}}=\frac{\mathrm{C}_{\mathrm{Hi}-1}-\frac{\mathrm{Q}_{\mathrm{GASi}} \cdot \chi_{\mathrm{H}}}{\mathrm{V}_{\mathrm{HSi}}} \Delta \mathrm{t}}{1+\frac{\left(\mathrm{Q}_{\mathrm{VIN}}+\mathrm{Q}_{\mathrm{GASi}}\right)}{\mathrm{V}_{\mathrm{HSi}}} \Delta \mathrm{t}}
$$

\subsubsection{Variations of Decant Model}

As well as simply decanting the tank, the effects of two postulated decant control strategies were investigated with the model. These control strategies included a stop-start control strategy and a water backfill.

Tank farm operations (CHG 2000) requires that operations be halted if the gas monitoring instruments measure a hydrogen concentration exceeding an level of $6,250 \mathrm{ppm}$ in the headspace. This control was set to prevent the concentration from reaching the LFL on the 
assumption that the operation was causing the gas release. Operations can theoretically resume when the hydrogen concentration drops back below 6,250 ppm. However, a lower restart threshold is typically used. The SY-101 mixer pump Safety Assessment (Sullivan 1995) specified a 500-ppm hydrogen concentration for restart. The only change in the model for the stop-start control strategy is to set the decant rate to zero when the hydrogen concentration rises above 6,250 ppm and restart the decant after the hydrogen concentration falls below $500 \mathrm{ppm}$.

Because decant-induced BDGREs result from decreasing hydrostatic pressure, it was believed that maintaining pressure by backfilling with water might prevent or reduce the frequency of BDGREs. However, because water is much less dense than the concentrated supernate the hydrostatic pressure cannot be maintained, and some potential for induced BDGREs remains. A negative side effect of the backfill is that the tank headspace does not increase (except minimally due to gas release) as supernate is removed. Only minor changes in the model were required for the water backfill control strategy tests. Water was added back to the tank at the same rate as the supernate was removed, so the waste level changes only with gas release.

$$
\mathrm{h}_{\mathrm{Wi}}=\sum_{\mathrm{t}=1}^{\mathrm{i}} \mathrm{h}_{\mathrm{Rt}}
$$

The pressure of the gas during the decanting process at a given depth in a gob (Eq. (3.1) is then given by

$$
P_{S i}(\eta)=P_{A}+\rho_{L_{i-1}} g\left(h_{C}+h_{L_{i}}+\eta h_{S}\right)+\rho_{W} g h_{W i}
$$

where $\rho_{\mathrm{w}}$ is the water density. The headspace volume (Eq. (3.32) is calculated by

$$
\mathrm{V}_{\mathrm{HSi}}=\mathrm{V}_{\mathrm{T}}-\left(\mathrm{h}_{\mathrm{C}}+\mathrm{h}_{\mathrm{Li}}+\mathrm{h}_{\mathrm{S}}+\mathrm{h}_{\mathrm{Wi}}\right) \mathrm{A}_{\mathrm{T}}
$$

\subsection{Parameter Definitions and Constraints}

Most of the model parameters define the initial physical conditions in the waste that can be obtained from directly measuring the waste layers. With few exceptions, all of these parameters have been established with reasonable accuracy and uncertainty for the tanks under evaluation. Parameters that describe the configuration of gas stored in the sediment are much more uncertain. The initial gas retention conditions must be defined by the number of gobs with each gob defined by its size, gas volume fraction, and vertical gas fraction profiles.

The behavior of the waste during decant is described by the rates of the various processes involved. Many of these parameters may be even more uncertain and difficult to define because the models in which they are used are also more inferred from indirect evidence than described from first principles. These parameters include coefficients for the gas release rates function, solids suspension fraction and the potential energy threshold. 
Each of these parameters, even those obtained directly from a measurement, has some uncertainty that can be described by a probability distribution. The bases for the parameter values for the four tanks under evaluation (AN-103, AN-104, AN-105, and AW-101) are presented in the following sections.

For those parameters requiring extensive analysis, AN-105 is presented in detail as the representative tank. It had the largest historic BDGREs next to SY-101, is one of the first low-activity waste tanks scheduled for retrieval, and is a good representative for the other BDGRE tanks in terms of physical and chemical composition. Model parameters for each of the tanks evaluated were developed in a similar fashion, and anomalies from this pattern are noted in each section.

\subsubsection{Overall Tank and Waste Configuration and Properties}

The tank and waste configuration properties include those parameters that are direct measurements or design specifications, as indicated in Table 3.3. Distributions for the measured parameters are summarized in Table 3.4. The maximum and minimum values are those to which the distributions are truncated. Complete probability distributions for each of the parameters for all tanks evaluated are given in Appendix C.

Table 3.3. Tank and Waste Configuration Parameters

\begin{tabular}{|c|c|c|c|}
\hline Parameter & Symbol & Source & Reference \\
\hline Tank Volume & $\mathrm{V}_{\mathrm{T}}$ & Design specification & \\
\hline Tank Area & $\mathrm{A}_{\mathrm{T}}$ & Design specification & \\
\hline Vent Rate & $\mathrm{Q}_{\mathrm{VIN}}$ & Operational measurement & \\
\hline Total Waste Thickness & $\mathrm{h}_{\mathrm{T}}$ & Enraf buoyancy gauge & $\begin{array}{l}\text { Hedengren et al. (2000), } \\
\text { Moore et al. (2000), } \\
\text { TWINS }^{\text {(a) }}\end{array}$ \\
\hline Crust Thickness & $\mathrm{h}_{\mathrm{C}}$ & Temperature profiles & $\begin{array}{l}\text { Hedengren et al. (2000), } \\
\text { Moore et al. (2000) }\end{array}$ \\
\hline Sediment Thickness & $\mathrm{h}_{\mathrm{S}}$ & Various measurements & $\begin{array}{l}\text { Hedengren et al. (2000), } \\
\text { Moore et al. (2000) }\end{array}$ \\
\hline Supernatant Liquid Density & $\rho_{\mathrm{L}}$ & $\begin{array}{l}\text { Ball rheometer, core } \\
\text { samples }\end{array}$ & $\begin{array}{l}\text { Hedengren et al. (2000), } \\
\text { Moore et al. (2000) } \\
\text { TWINS }^{\text {(a) }}\end{array}$ \\
\hline Sediment Density & $\rho_{\mathrm{S}}$ & Core samples & $\begin{array}{l}\text { TWINS, }{ }^{(a)} \text { Hedengren et } \\
\text { al. }(2000) \text {, Moore et al. } \\
(2000)\end{array}$ \\
\hline $\begin{array}{l}\text { Hydrogen Concentration in gas } \\
\text { stored in Sediment Layer }\end{array}$ & $\chi_{\mathrm{H}}$ & $\begin{array}{l}\text { Retained gas sampler (RGS) } \\
\text { measurement }\end{array}$ & Mahoney et al. (1999) \\
\hline Hydrogen Generation Rate & $\mathrm{H}_{2 \mathrm{G}}$ & Empirical model & $\mathrm{Hu}(2000)$ \\
\hline $\begin{array}{l}\text { Initial Gas Volume in Sediment } \\
\text { Layer }\end{array}$ & $\mathrm{V}_{\mathrm{G} 0}$ & $\begin{array}{l}\text { Void fraction instrument } \\
\text { (VFI) and RGS } \\
\text { measurements }\end{array}$ & Hedengren et al. (2000) \\
\hline Sediment Yield Stress & $\tau_{\mathrm{y}}$ & Ball rheometer & Hedengren et al. (2000) \\
\hline
\end{tabular}


Table 3.4. Tank and Waste Configuration Parameters: Values and Distributions

\begin{tabular}{|c|c|c|c|c|c|}
\hline $\begin{array}{l}\text { Parameter, } \\
\text { Distribution } \\
\text { (units) }\end{array}$ & & AN-105 & AN-104 & AN-103 & AW-101 \\
\hline $\begin{array}{c}\mathrm{V}_{\mathrm{T}} \\
\left(\mathrm{m}^{3}\right)\end{array}$ & Point Value & 5337.4 & 5337.4 & (5337.4 & $\begin{array}{c}5337.4 \\
\end{array}$ \\
\hline $\begin{array}{c}\begin{array}{c}\mathrm{A}_{\mathrm{T}} \\
\left(\mathrm{m}^{2}\right)\end{array} \\
\end{array}$ & Point Value & 410.4 & 4110.4 & 4110.4 & 410.4 \\
\hline $\begin{array}{l}\mathrm{Q}_{\mathrm{VIN}} \\
\text { (cfm) }\end{array}$ & Point Value & 100 & 100 & 100 & 125 \\
\hline \multirow{4}{*}{$\begin{array}{c}\mathrm{h}_{\mathrm{T}} \\
\text { normal } \\
(\mathrm{m})\end{array}$} & Median & 10.41 & 9.72 & 8.84 & 10.4 \\
\hline & Maximum & 10.51 & 9.82 & 8.94 & 10.5 \\
\hline & Minimum & 10.31 & 9.62 & 8.74 & 10.3 \\
\hline & $3 \sigma$ & 0.10 & 0.10 & 0.10 & 0.10 \\
\hline \multirow{3}{*}{$\begin{array}{c}\mathrm{h}_{\mathrm{C}} \\
\text { uniform } \\
(\mathrm{m})\end{array}$} & Median & 0.45 & 0.41 & 0.89 & 0.80 \\
\hline & Maximum & 0.65 & 0.55 & 1.21 & 1.20 \\
\hline & Minimum & 0.25 & 0.27 & 0.57 & 0.40 \\
\hline \multirow{4}{*}{$\begin{array}{c}\mathrm{h}_{\mathrm{S}} \\
\text { normal } \\
(\mathrm{m})\end{array}$} & Median & 4.50 & 4.05 & 3.78 & 2.86 \\
\hline & Maximum & 5.34 & 4.85 & 3.92 & 3.46 \\
\hline & Minimum & 3.66 & 3.25 & 3.64 & 2.26 \\
\hline & $2 \sigma$ & 0.84 & 0.80 & 0.14 & 0.60 \\
\hline \multirow{4}{*}{$\begin{array}{c}\rho_{\mathrm{L}} \\
\text { normal } \\
\left(\mathrm{kg} / \mathrm{m}^{3}\right)\end{array}$} & Median & 1430 & 1440 & 1450 & 1430 \\
\hline & Maximum & 1520 & 1548 & 1544 & 1516 \\
\hline & Minimum & 1340 & 1332 & 1356 & 1344 \\
\hline & $2 \sigma$ & 90 & 108 & 94 & 86 \\
\hline \multirow{4}{*}{$\begin{array}{c}\rho_{\mathrm{s}} \\
\text { normal } \\
\left(\mathrm{kg} / \mathrm{m}^{3}\right)\end{array}$} & Median & 1572 & 1579 & 1737 & 1553 \\
\hline & Maximum & 1652 & 1679 & 1957 & 1633 \\
\hline & Minimum & 1492 & 1479 & 1517 & 1473 \\
\hline & $2 \sigma$ & 80 & 100 & 220 & 80 \\
\hline \multirow{4}{*}{$\begin{array}{c}\chi_{\mathrm{H}} \\
\text { normal }\end{array}$} & Median & 0.59 & 0.45 & 0.61 & 0.32 \\
\hline & Maximum & 0.644 & 0.519 & 0.687 & 0.352 \\
\hline & Minimum & 0.536 & 0.381 & 0.533 & 0.288 \\
\hline & $3 \sigma$ & 0.054 & 0.069 & 0.077 & 0.032 \\
\hline $\begin{array}{c}\begin{array}{c}\mathrm{H}_{2 \mathrm{G}} \\
\left(\mathrm{ft}^{2} / \text { day }\right)\end{array} \\
\end{array}$ & Point Value & 3.32 & 4.97 & 4.26 & 4.80 \\
\hline \multirow{2}{*}{$\begin{array}{c}\mathrm{V}_{\mathrm{G} 0} \\
\left(\mathrm{~m}^{3}\right) \\
\end{array}$} & Maximum & 100 & 99 & 183 & 51 \\
\hline & Minimum & 60 & 64 & 157 & 39 \\
\hline \multirow{2}{*}{$\begin{array}{c}\tau_{\mathrm{y}} \\
\text { uniform } \\
(\mathrm{Pa}) \\
\end{array}$} & Maximum & 250 & 250 & 250 & 250 \\
\hline & Minimum & 50 & 50 & 50 & 50 \\
\hline
\end{tabular}




\subsubsection{Gob Size and Number}

As discussed, the preponderance of evidence requires the sediment layer to be considered as a collection of semi-independently evolving gobs of varying sizes with different degrees of gas retention and relative buoyancy (Meyer et al. 1997; Meyer and Stewart 2001). The volume of gas released from a gob is determined by the size of the gob, the gas volume within the gob, and the fraction of gas that is released during a BDGRE. Inversely, therefore, the size of a gob may be determined from the volume of gas released during a BDGRE.

The hydrogen concentration in a tank's headspace can be used to quantify gas release behavior during BDGREs (Hedengren et al. 2000). Gas release volumes have been determined from historical BDGREs as presented in Section 2.3. From Eq. (3.24), the release volume for a gob can be expressed as

$$
\mathrm{V}_{\mathrm{re} \ln }=\frac{\mathrm{Q}_{\mathrm{R} 0} \tau_{\mathrm{n}}}{\chi_{\mathrm{H}}}
$$

The gob volume can then be estimated by combining Eq. (3.20) and (3.21) to yield

$$
\mathrm{V}_{\text {gobn }}=\frac{\mathrm{V}_{\text {re ln }}}{\mathrm{P}_{\mathrm{S}} \alpha_{\mathrm{NB}} \mathrm{f}_{\text {rel }}}
$$

Recall that the analysis of the BDGREs for this study departed from previous analyses (Hedengren et al. 2000, for example) in that the possibility of multiple BDGREs was allowed for in events that were previously thought to be single BDGREs. The identification of multiple BDGRE phenomena is discussed in detail in Section 2.3.

Combining Eq. (3.20) and (3.44) yields the following expression for the gob diameter based on the gas release volume:

$$
\mathrm{D}_{\mathrm{n}}=\sqrt{\frac{4}{\pi} \frac{\mathrm{V}_{\mathrm{reln}}}{\mathrm{P}_{\mathrm{S}} \alpha_{\mathrm{NB}} \mathrm{f}_{\mathrm{rel}} \mathrm{h}_{\mathrm{S}}}}
$$

The gob diameters determined for AN-105 (including single and multiple release events) in this manner are shown in Figure 3.4. 


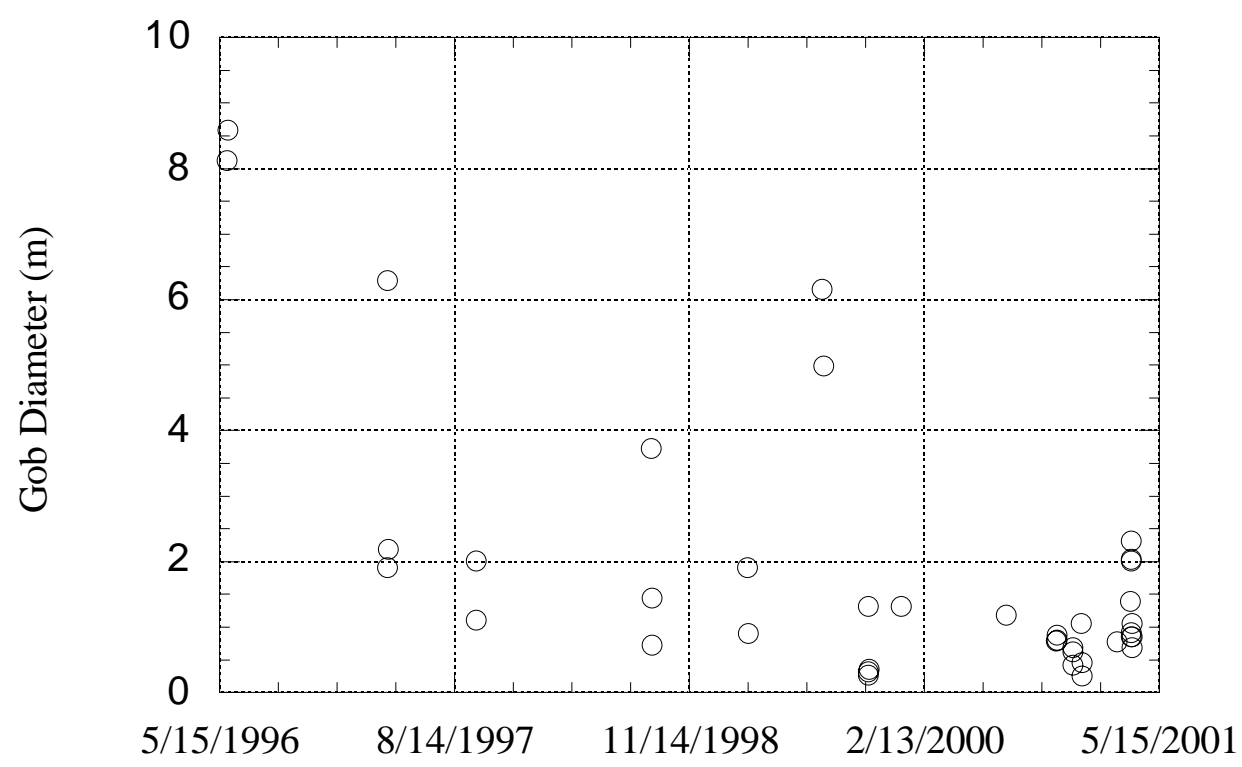

Figure 3.4. Tank AN-105 BDGRE Gob Diameters

It appears that BDGRE gob volumes have been decreasing in AN-105 over the period shown. Similar trends are seen in the other tanks. A gob diameter distribution taken directly from this data would have the highest probability at smaller $(\sim 1 \mathrm{~m}$ diameter $)$ gob sizes. However, a Rayleigh-Taylor stability analysis conducted by Meyer et al. (1997) indicates that gobs with aspect ratios near unity are the least stable. Given our assumption that a gob is represented by a right-circular cylinder with a depth approximately equal to the sediment thickness (supported by the gas fraction profiles), the gob diameters would most probably be closer to the sediment thickness and therefore larger ( $4.5 \mathrm{~m}$ diameter). Further, the gobs must be relatively large given the gas generation rate and event frequency and size. This may be seen by considering that about $5201-\mathrm{m}$-diameter gobs could be present in a tank. The frequency of events seen in the tanks (Hedengren et al. 2000) clearly does not support this distribution. Finally, it has been determined that it is also more conservative to consider larger gobs.

Thus a gob diameter distribution was chosen that would allow for both small and large gobs but has the most probable gob diameter as the sediment layer depth to approximately the largest historical value. The probability decreases at either end of the distribution as dictated by the tank size, indicating a maximum diameter of $22 \mathrm{~m}$ and a minimum of zero. The diameter values were truncated to $11 \mathrm{~m}$ (tank radius) and $1 \mathrm{~m}$. The detailed diameter distributions for each tank are presented in Appendix C. The single peak in AN-103 is achieved because the sediment depth and largest historical gob sizes are equivalent.

Based on combinations of the gas release history and generation rates, and sediment layer volume and gob volume (Meyer et al. 1997), it is estimated that there are approximately 10 to 15 gobs in a tank. The actual number of gobs (gob count) in the tank is determined by the limit of the tank area, or 


$$
\sum_{n=1}^{\text {gob }}{ }_{n}^{\text {count }} \frac{\pi}{4} \mathrm{D}_{\mathrm{n}}^{2}=\mathrm{A}_{\mathrm{T}}
$$

with $\mathrm{D}_{\mathrm{n}}$ randomly selected from its distribution until all of the tank area is occupied. Because the assumed shape is an approximation and the geometric location of an individual gob is irrelevant in this analysis, the non-physical complete occupation of the circular tank area by circular gobs is ignored. When the remaining tank area is less than that that determined by the minimum gob diameter, the final gob diameter is set by

$$
\mathrm{D}_{\mathrm{n}}=\sqrt{\frac{4}{\pi} \mathrm{A}_{\mathrm{T}}-\sum_{\mathrm{n}=1}^{\text {gob_count }-1} \mathrm{D}_{\mathrm{n}}^{2}}
$$

\subsubsection{Gob Gas Fraction and Gas Fraction Profile}

The total gas volume in the sediment layer must be inferred from local gas fraction measurements. The initial average gas fraction in a gob is necessarily less than the neutral buoyant gas fraction and is assumed to be greater than approximately $1 / 4$ of this value. This minimum can be computed by the release fraction of gas from a gob and the in situ hydrostatic pressure in the sediment layer. For our purposes, given that low-gas fraction gobs will not be affected by a decant, a fixed minimum is used that is approximately $1 / 4$ of a minimum neutral buoyant gas fraction (dictated by the layer densities [Table 3.4] and Eq. 3.11) for the particular tank. The average gas fraction of an individual gob is then randomly selected from the ranges presented in Table 3.5.

Table 3.5. Gas Fraction Ranges

\begin{tabular}{|c|c|c|c|c||}
\hline \hline Gas Fraction & AN-105 & AN-104 & AN-103 & AW-101 \\
\hline Minimum & 0.02 & 0.02 & 0.04 & 0.02 \\
\hline Maximum & $\alpha_{\mathrm{NB}}$ & $\alpha_{\mathrm{NB}}$ & $\alpha_{\mathrm{NB}}$ & $\alpha_{\mathrm{NB}}$ \\
\hline
\end{tabular}

The distribution shape of the initial average gas fraction is dictated by the initial gas volume in the sediment layer. The assignation of gas fraction to the gob volumes is made by

$$
\sum_{\mathrm{n}=1}^{\text {gob }} \alpha_{\mathrm{A} 0 \mathrm{n}} \lambda \frac{\pi}{4} \mathrm{D}_{\mathrm{n}}^{2} \mathrm{~h}_{\mathrm{S}}=\mathrm{V}_{\mathrm{G} 0}
$$

where $\alpha_{\mathrm{A} 0 \mathrm{n}}$ is the initial average gas fraction in the gob, and $\lambda$ is a factor that accounts for the vertical gas fraction profile in a gob (see below). A set of gas fractions that satisfy Eq. (3.48) are assigned to a set of gob diameters that meet the constraint of Eq. (3.46).

To have a gas volume from the gob configuration that matched the total gas volume in the sediment layer (Table 3.4), trapezoidal distributions for the gas fractions were used. Higher probabilities were assigned to the minimum gas fractions for each tank (compared with the 
probability of $\alpha_{\mathrm{NB}}$ ) except AN-103, where a much higher probability of $\alpha_{\mathrm{NB}}$ had to be assigned. This apparent deviation of $\mathrm{AN}-103$ from the gas retention characteristics in the other tanks is supported by the lack of BDGREs in AN-103, the small size of those that do occur, and the slow level growth in the tank (Hedengren et al. 2000).

The rate of buoyancy and the volume of the gob that becomes buoyant are affected by the vertical gas fraction profile in a gob (see Section 3.1.1). This profile also affects the computation of the total gas volume in the tank (Eq. 3.48). Recall that the average gas fraction of interest in regard to buoyant conditions during a decant in a parabolic gas fraction profile gob is that at $3 / 4$ $\mathrm{h}_{\mathrm{s}}$. The average gas fraction for a parabolic profile gob selected from the distribution range is therefore taken to be this average value, which is higher than the average gas fraction over the entire depth of the gob (see Figure 3.2). The relationship between the average gas fraction from the top to $3 / 4$ of the way down into the gob to that over the entire gob can be computed by

$$
\alpha_{\mathrm{A} 0 \mathrm{n}}(\text { entire_gob })=\frac{16}{3} \alpha_{\mathrm{A} 0 \mathrm{n}} \int_{0}^{1} \eta(1-\eta) \mathrm{d} \eta=0.889 \alpha_{\mathrm{A} 0 \mathrm{n}}
$$

A binary distribution of either linear or parabolic gas fraction profile is used (Hedengren et al. 2000). For those gobs that have a parabolic profile, $\lambda$ in Eq. (3.48) is set to 0.889, and for linear profile gobs, 1.0 .

An interesting effect of the inferred gas volume is that it reduces the possible range of the initial gas fraction in the gobs for each of the tanks, thereby reducing the variability of the liquid and sediment densities (see Eq. 3.11). This effect is reflected in the parameter distributions presented in Appendix C.

\subsubsection{Gas and Solid Release Rate}

Gas is released from the gob at the same time-varying rate as it is during spontaneous BDGREs (see Section 3.1.2). The headspace hydrogen concentration recorded during spontaneous BDGREs in each of the tanks under evaluation has been modeled by Eq. (3.24) and (3.37) in Section 2.4. This analysis has provided the time to the peak release rate $\left(\tau_{\mathrm{n}}\right)$ required in Eq. (3.26) and (3.27), and the results for AN-105 are shown in Figure 3.5. No specific trend is observable over the time period (approximately six years), and no correlation is observable between $\tau$ and the release volume from given events. It is apparent that, even neglecting the uncertainty of the analysis, no definitive $\tau$ value is identifiable. Similar results were observed in the other tanks. As a result, the $\tau$ value for a gob in a given tank was assigned from a distribution created from that tank's historical release results, as shown in Appendix C.

As discussed, a fraction of the portion of the gob that disintegrates in the gas release process is assumed to remain suspended and become mixed in the supernate and not resettle with the rest of the spent gob. The fraction of this released material that remains suspended in the supernate, $\mathrm{F}$ in Eq. (3.29), is determined from the historical headspace hydrogen concentration data. 


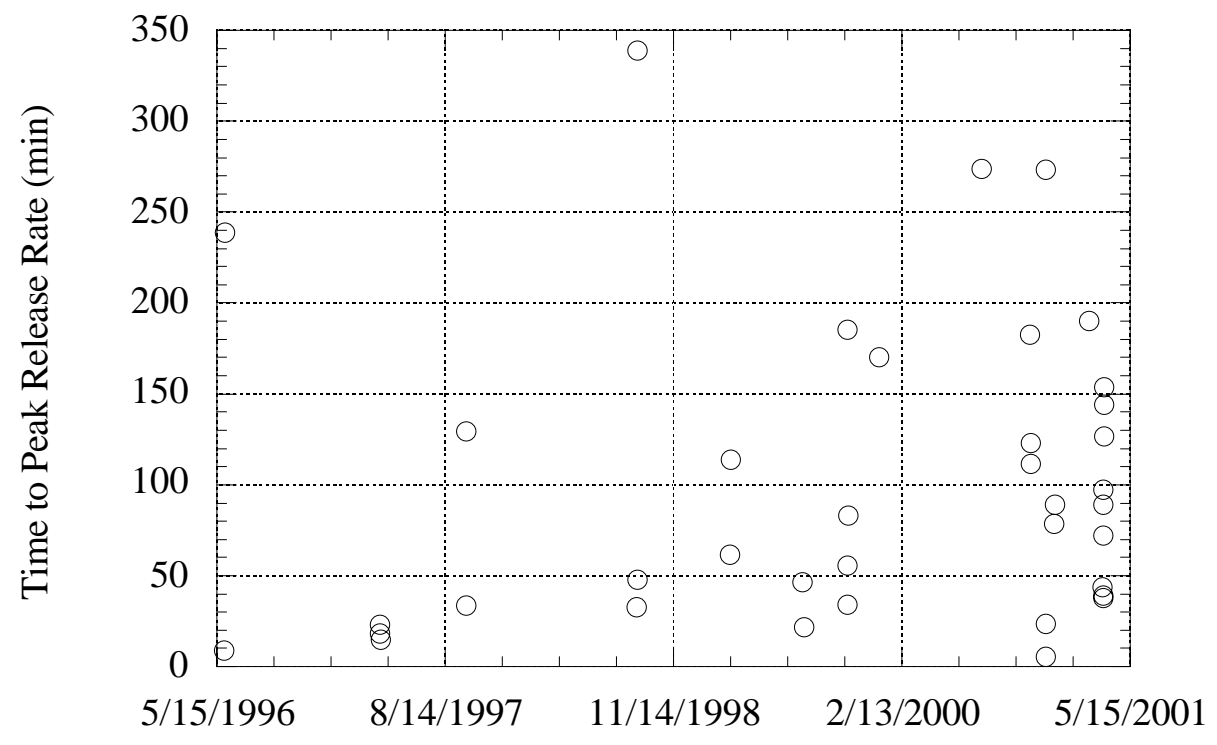

Figure 3.5. Tank AN-105 Time to Peak Release Rates for Spontaneous BDGREs

Numerous historical (spontaneous) BDGRE events have multiple-gob participation (Section 2.3). We have assumed that this multiple gob phenomenon is directly and solely influenced by the suspension of the released material. Therefore, to quantify F, the multiple gob behavior has been described by specific parameters:

- The number of gobs participating in a GRE.

- The fraction of the gas released. This parameter is computed by dividing the total gas release volume of a single or multiple GRE by the total amount of gas stored in the sediment layer.

- The fraction of the tank area participating in BDGREs. This parameter is computed by dividing the total gob area of a single or multiple GRE by the total tank area. The participating gob area is determined directly from the gas release volumes, as discussed in Section 3.2.2.

- The time between gobs.

The results of the spontaneous BDGREs are compared with results generated from a modified decant model. To account for the variability of the input conditions, approximately 400 different sets of inputs were created from the input parameter distributions discussed above. For each input case, a supernatant decant was conducted until a BDGRE was triggered; at that point the decant was stopped. Care was taken in the selection of the input cases to ensure that the initial BDGRE was triggered during the early stages of the decant operation so that the effects of the decant were minimized (reduced supernatant liquid volume, reduced hydrostatic pressure, increased headspace, etc.) to replicate the tank conditions during a spontaneous BDGRE. For each input case, eight values of $\mathrm{F}$ were used, ranging from the physically imposed limits of zero 
to one in a geometric progression and resulting in approximately 3,200 simulations. Comparing the spontaneous BDGRE results to the model BDGRE results indicated applicable values for F.

Spontaneous BDGREs for each tank were selected from those in Table 2.4. Individual events with a total gas release volume greater than $1 \mathrm{~m}^{3}$ were included in the evaluation. This imposed release volume limit follows from our selection of potential gob diameters discussed in Section 3.2.2. The parameter values determined from the spontaneous events are given in Table 3.6, and model parameter results as functions of $\mathrm{F}$ are presented in Figures 3.6 through 3.9.

Table 3.6. Spontaneous BDGRE Behavior Parameters

\begin{tabular}{|c|c|c|c|c|c||}
\hline \hline Parameter & & AN-105 & AN-104 & AN-103 & AW-101 \\
\hline \hline \multirow{2}{*}{$\begin{array}{c}\text { Number of } \\
\text { Gobs }\end{array}$} & Maximum & 3 & 3 & 2 & 4 \\
\cline { 2 - 6 } & Average & 1.4 & 1.4 & 1.3 & 2 \\
\cline { 2 - 6 } & Minimum & 1 & 1 & 1 & 1 \\
\hline \hline \multirow{2}{*}{$\begin{array}{c}\text { Fraction of } \\
\text { Gas }\end{array}$} & Maximum & 0.322 & 0.111 & 0.014 & 0.144 \\
\cline { 2 - 6 } Released & Average & 0.073 & 0.039 & 0.008 & 0.091 \\
\cline { 2 - 6 } & Minimum & 0.008 & 0.004 & 0.005 & 0.020 \\
\hline \hline \multirow{2}{*}{$\begin{array}{c}\text { Fraction of } \\
\text { Tank Area } \\
\text { Participating }\end{array}$} & Maximum & 0.267 & 0.148 & 0.023 & 0.114 \\
\cline { 2 - 6 } & Average & 0.061 & 0.052 & 0.015 & 0.072 \\
\hline \hline \multirow{2}{*}{$\begin{array}{c}\text { Time } \\
\text { Between } \\
\text { Gobs (hrs) }\end{array}$} & Minimum & 0.007 & 0.006 & 0.008 & 0.016 \\
\cline { 2 - 6 } & Maximum & 31.9 & 8.3 & 37.6 & 7.9 \\
\cline { 2 - 6 } & Average & 14.7 & 3.9 & 37.6 & 3.7 \\
\hline \multirow{2}{*}{} & & 3.3 & 0.5 & 37.6 & 1.7 \\
\hline
\end{tabular}

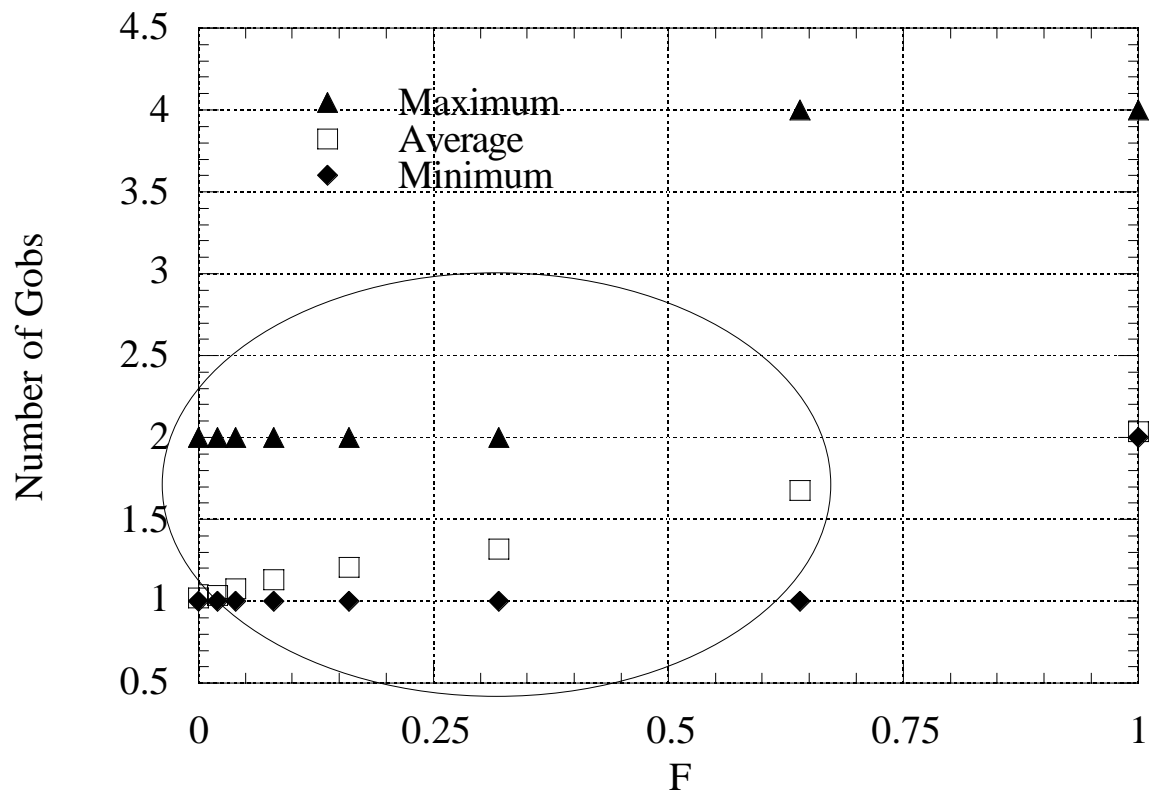

Figure 3.6. Number of Gobs as a Function of F: Model Results, AN-105 


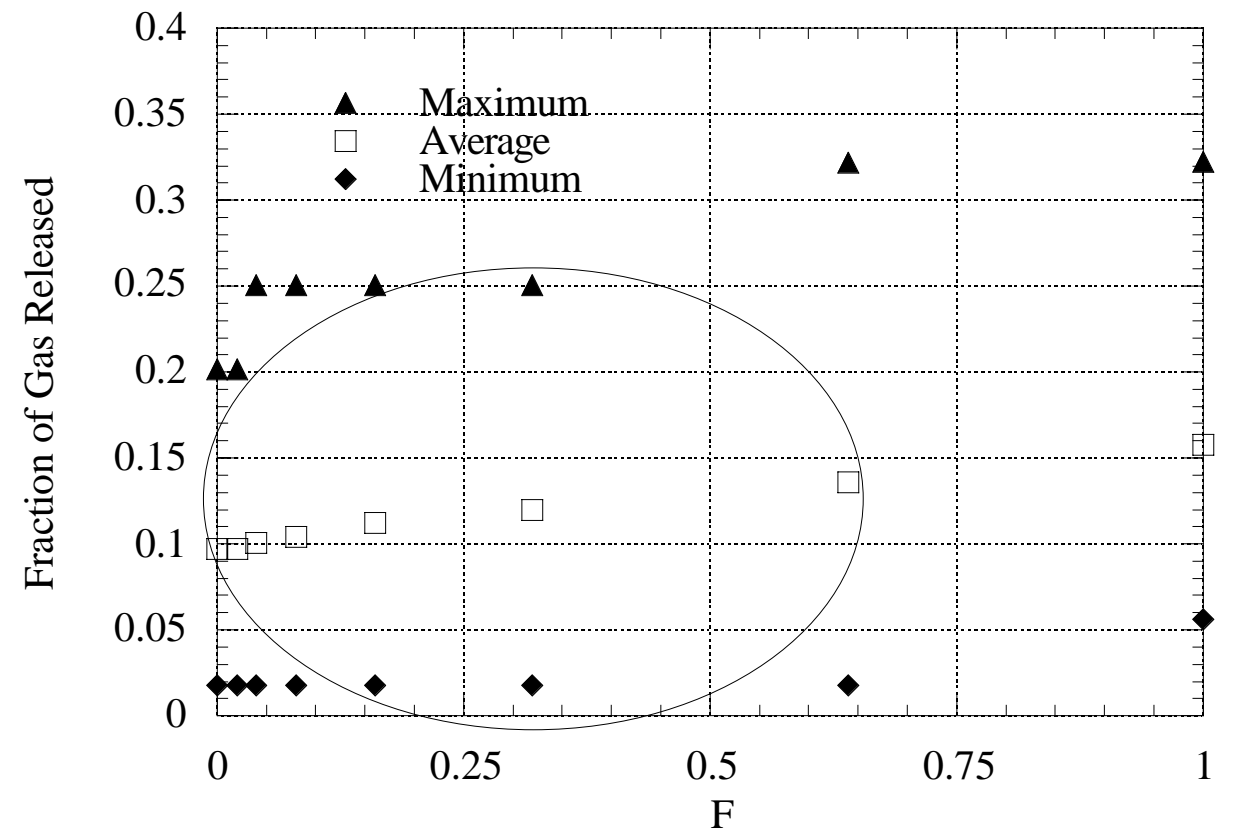

Figure 3.7. Fraction of Gas Released as a Function of F: Model Results, AN-105

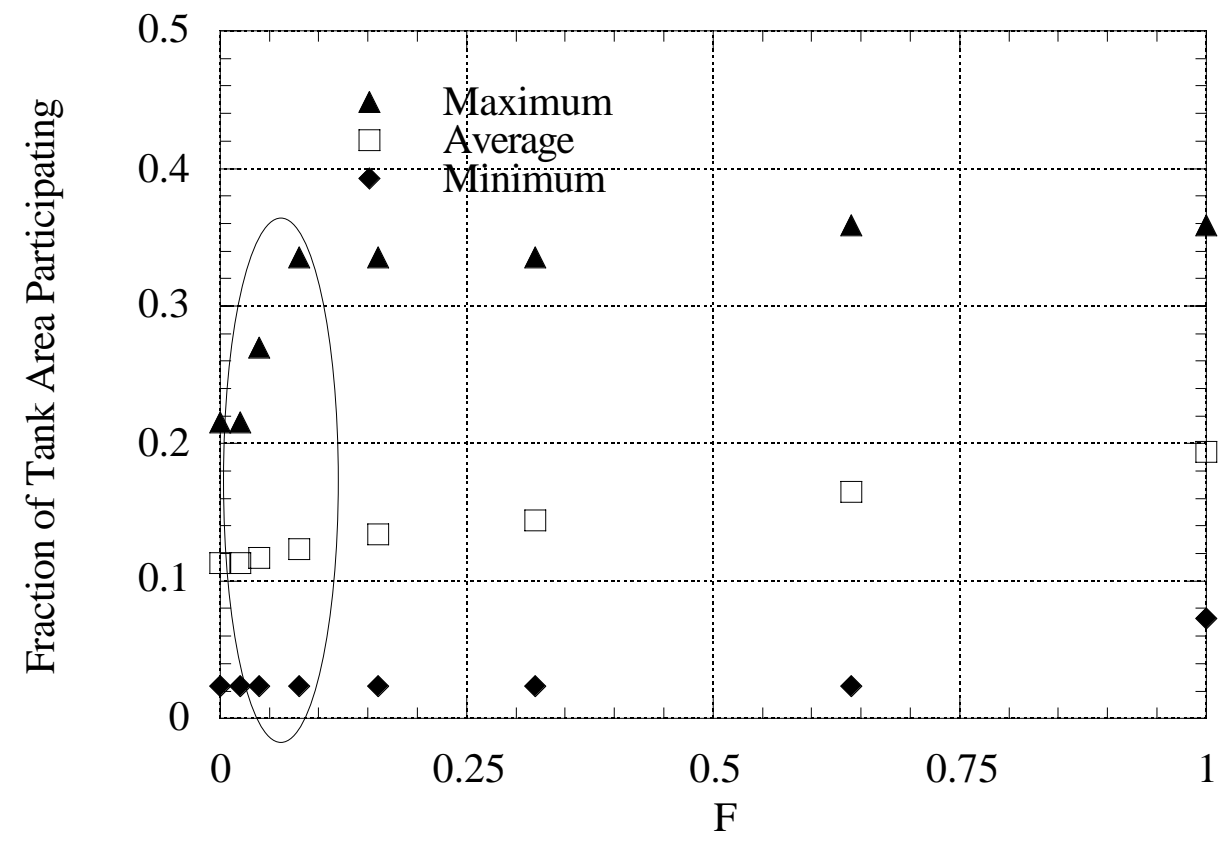

Figure 3.8. Fraction of Tank Area Participating as a Function of F: Model Results, AN-105 
For each parameter, the appropriate F values as determined by the model are based on the results that do not exceed the spontaneous BDGRE results. That is, at what value of $F$ do the model results exceed the spontaneous results? The necessity of not exceeding the spontaneous results is clearly illustrated by the minimum number of gobs. Comparison of the minimum number of gobs in Table 3.6 with those in Figure 3.6 indicates that F values at least 0.64 and below produce single gob GREs. Because single gob GREs have been identified in the historical tank data, setting $\mathrm{F}$ above 0.64 would not represent actual tank behavior. Likewise, the comparison of the average number of gobs indicates that $\mathrm{F}$ values of approximately 0.5 are bounding. At this value of $\mathrm{F}$, the maximum number of gobs is not exceeded. However, the occurrence of single events is much more certain than the determination of the average number. Therefore, based on the number of gobs in a GRE, F may be bounded at or below approximately 0.6. A similar process was conducted for each of the remaining parameters. On each plot, the bounding range of $\mathrm{F}$ is indicated by the ellipsoid.

F appears to be bounded at or below 0.64 by the fraction of gas released (Figure 3.7), at or below 0.1 by the fraction of tank area participating (Figure 3.8), and below 0.1 by the time between gobs (Figure 3.9). Based on the inherent uncertainty in the determination of the parameters for the spontaneous BDGREs and the general behaviors observed in the simulation results, no definitive $\mathrm{F}$ values can be identified. We have therefore selected a distribution which has the most probable $F$ values between 0.1 and 0.6 , and the probability decreases at either end of the distribution to the physical limits of zero and unity. This distribution is used for each of the tanks, and the input probability distributions are shown in Appendix C.

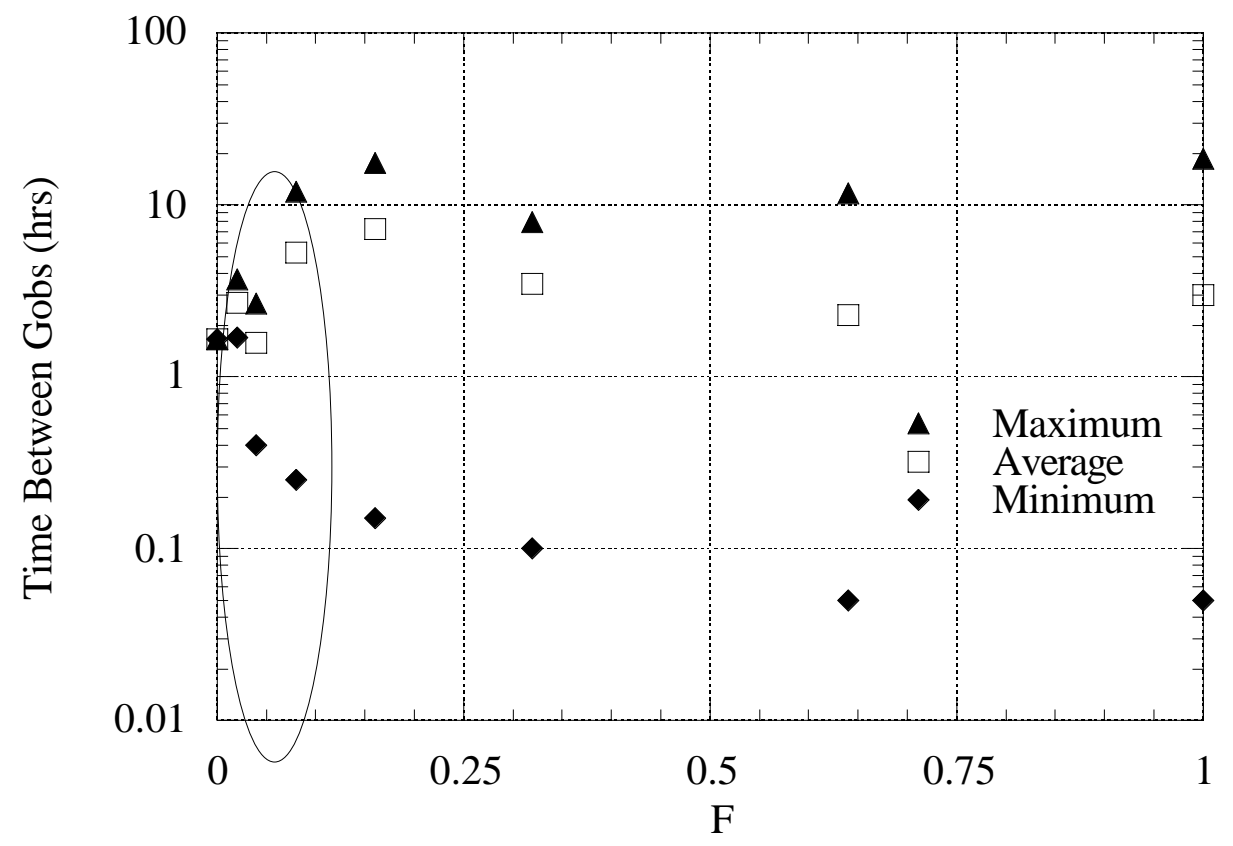

Figure 3.9. Time Between Gobs as a Function of F: Model Results, AN-105 
It is interesting to consider the relation between the time between gobs and the time to the peak release rate. Given our assumption that multiple BDGREs occur due to the suspension of solids into the supernate at the same rate as the gas release, it is reasonable to expect that, as the time to the peak release rate is increased (slower buildup of solids in the supernate), the time between gobs would also increase. This is reflected in the selected spontaneous BDGRE data (selected as above) for all four tanks as shown in Figure 3.10. While it is certainly not conclusive, this behavior does support our assumptions for gob interaction mechanisms.

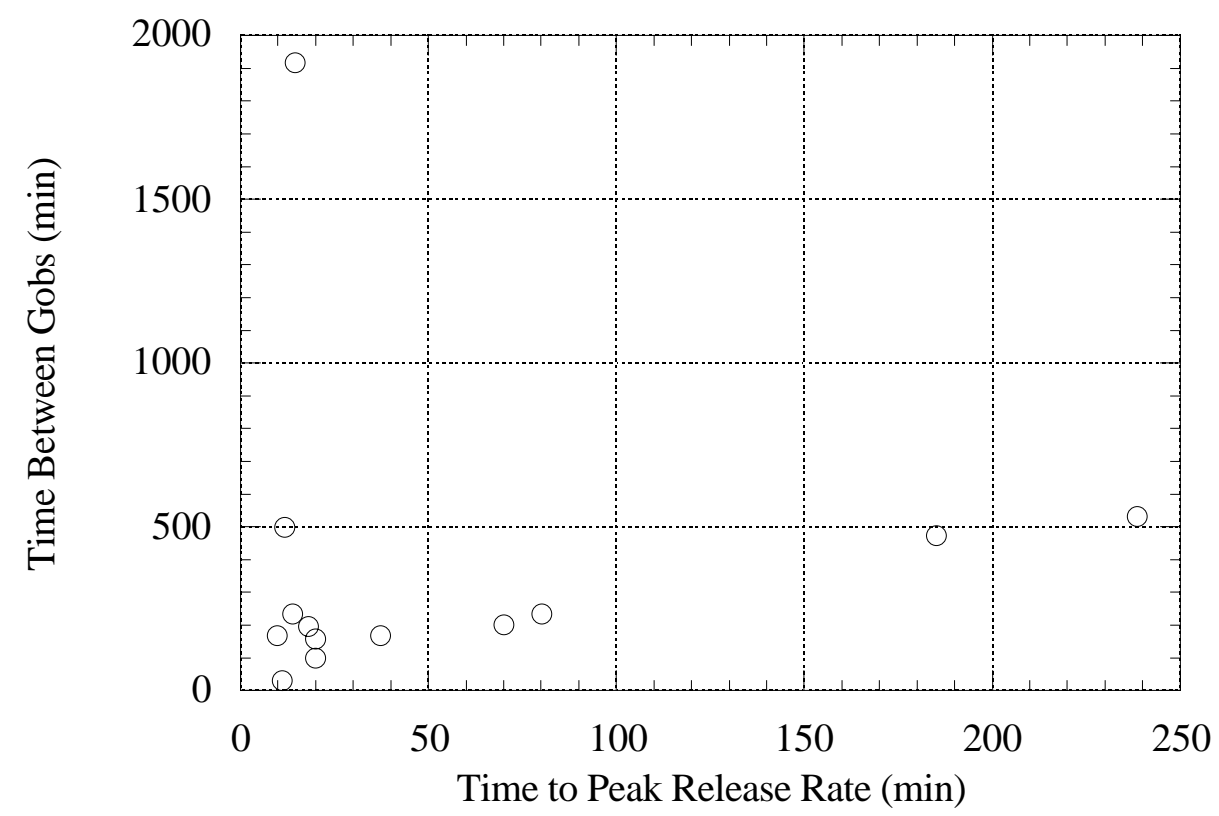

Figure 3.10. Time Between Gobs as a Function of the Time to Peak Release Rate

\subsubsection{Monte Carlo Simulation Method}

From the preceding discussion of the input parameters, it is apparent that the majority have significant, quantified uncertainties. A deterministic calculation with all parameters set to bounding values has no physical or statistical meaning. Instead, we must account for all the parameter uncertainties and establish the overall probability distribution for the model's predictions. To accomplish this, a large number of model simulations are run with input parameter sets selected from their respective distributions. The collection of output values from all the model simulations then forms the desired overall probability distribution.

A Monte Carlo simulation approach was used. This approach can be employed to determine the uncertainty of modeling results when the input parameters have uncertainty distributions. For each retrieval operation modeled, 10,000 simulation runs were conducted. Simulating a large number of cases allows all important physical effects included in the model to influence the predicted behavior. The result is a set of 10,000 model outputs, each with its own set of predicted results, that constitutes a probability distribution over those predicted results. This allows us to predict the probability of a given result given the input probability distributions. 
Each value used for the inputs is randomly sampled from an infinite population based on the specified distribution. The commercial code $\mathrm{S}+$ was used to generate these inputs. In addition to the constraints specified above, the liquid and sediment layer densities were selected from the same half of their respective distributions (i.e., a liquid density above its median required a sediment density above its respective median, or vice-versa). Ten-thousand input distributions that met all of the constraints were created for each retrieval operation modeled. A Visual Basic code written around the Excel $^{\circledR}$-based model was used to generate results for each of the 10,000 simulations.

\subsection{Analysis of GREs Induced by Supernate Decant in AN-105}

While supernate decant simulations were performed for each of the four tanks of interest (AN-103, AN-104, AN-105, and AW-101), Tank AN-105 was selected to be presented in detail because it has experienced BDGREs with the highest headspace hydrogen concentration, is one of the first tanks scheduled to be retrieved for vitrification, and it was selected as representative of the other tanks.

Four cases were run to investigate the sensitivity of the results to the decant rate and the effects of two postulated control strategies:

1. Base Case: The decant rate was set at a constant $200 \mathrm{gpm}$. The model was run until essentially the entire supernatant layer was removed. The 200-gpm decant rate was chosen as the maximum flow rate likely to be achieved by available transfer pumps.

2. Decant Rate Sensitivity: The decant rate was reduced to $30 \mathrm{gpm}$. A lower decant rate was expected to reduce the peak hydrogen concentration. The 30-gpm flow rate was selected as the minimum practical decant rate.

3. Stop-Start Control Strategy: The base case was rerun with logic to stop the decant when the headspace hydrogen concentration exceeded $6,250 \mathrm{ppm}$ and to restart decant when the hydrogen concentration fell below $500 \mathrm{ppm}$. This was postulated as a means to reduce the peak hydrogen concentrations by preventing back-to-back BDGREs. A hydrogen concentration of $6,250 \mathrm{ppm}$ is the action level for gas monitoring controls (CHG 2000); 500 ppm was the restart point in the SY-101 mixer pump tests (Sullivan et al. 1995).

4. Water Backfill Control Strategy: The base case was rerun with logic to add water at the same rate as decant, assuming that the water did not mix with the supernate. This was postulated as a method to maintain the hydrostatic pressure and prevent BDGREs.

Results of each of these cases for AN-105 are presented in separate sections. The method of presentation of the results is explained in Section 3.3.1. Results of the base case are shown in Section 3.3.2 and the decant sensitivity study in Section 3.3.3. The Start-Stop and Water Backfill control strategies are presented in Sections 3.3.3 and 3.3.4, respectively. The overall conclusions of these studies are summarized in Section 3.3.5. The results of the simulations for the other three tanks are given in Section 3.4. 


\subsubsection{Method of Presenting Analysis Results}

As described in Section 3.2, the Monte Carlo simulation method propagates the probability distributions for the input parameters through the model to produce probability distributions of the results. This is done by performing a large number of simulations with different sets of parameter values chosen at random from the input probability distributions. It was found that 10,000 simulations were adequate to accurately represent the full range of inputs.

Each of these 10,000 simulations consists of a single supernate decant operation from start to finish, nominally calculating 1,000 time steps of 3 minutes each for a total of 50 hours. At each time step, data are recorded describing the state of the tank, including the condition of each gob. These data allow, for example, the headspace hydrogen concentration to be plotted as a function of time through the entire operation. This is shown schematically in Figure 3.11.

\section{Hydrogen Concentration Data from 10,000 Runs}

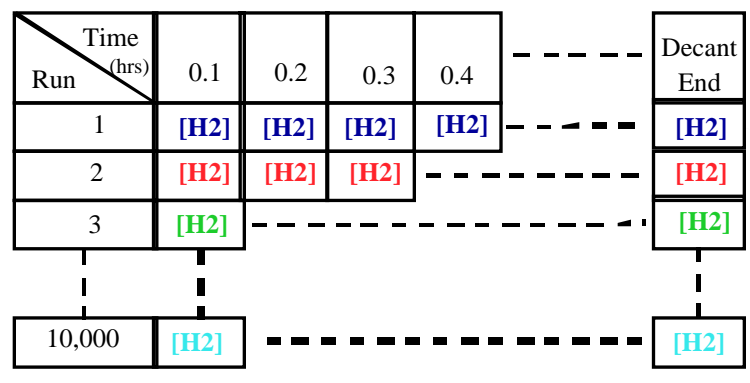

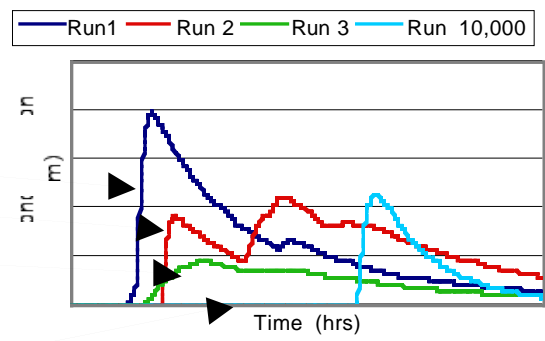

\section{Results of Four Runs Plotted vs. Time}

Figure 3.11. Schematic of Hydrogen Concentration Data

The peak hydrogen concentration attained during decant is the most useful result for assessing the flammable gas hazard. A set of 10,000 peak hydrogen concentrations is created from the results of the 10,000 Monte Carlo simulations performed for each case, each produced by a different set of input parameters. These data are sorted into 100 uniform bins to produce a fine-scale histogram describing the probability distribution for the peak hydrogen concentration. This process is represented by the sketch in Figure 3.12.

The frequency and cumulative frequency are plotted with the median and the 95th percentile values (95\% confidence limit [CL]) identified by a dashed line. These probability distributions are plotted for both the peak hydrogen concentration and the fraction of gas in the sediment layer released during decant. The transient hydrogen concentrations for one or two representative runs are also plotted to better illustrate the dynamic gas release behavior and to show the sequence of BDGREs. The median and 95th percentile values for the base case are shown on the three succeeding cases to illustrate the effect of the changes involved. 
Peak $\left[\mathrm{H}_{2}\right]$ from $\left[\mathrm{H}_{2}\right](\mathbf{t})$

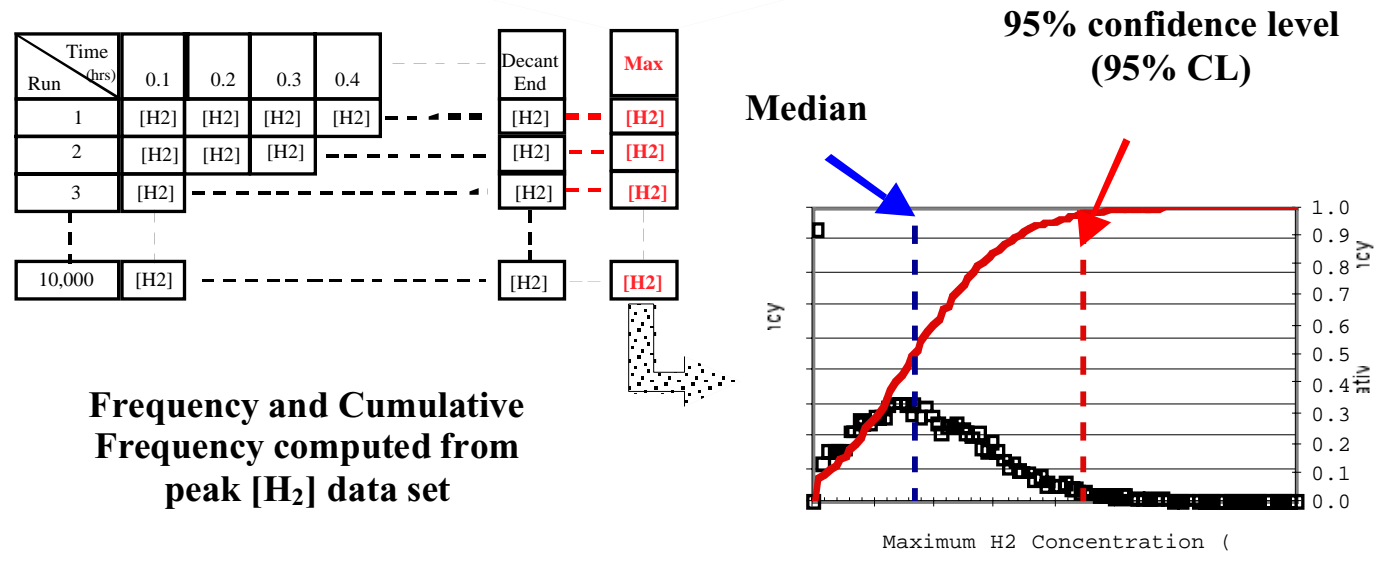

Figure 3.12. Derivation of Output Probability Distribution

\subsubsection{Base Case}

The distribution of peak hydrogen concentration for the base case is shown in Figure 3.13. This case used a constant 200-gpm decant rate with $100 \mathrm{scfm}$ of ventilation. The median of the peak hydrogen concentration data set is $2,800 \mathrm{ppm}$, and the 95 th percentile value is $8,300 \mathrm{ppm}$, lower than $25 \%$ of the LFL in AN-105 but higher than the action level of 6,250 ppm. The initial high value of the frequency indicates that $14.4 \%$, or 1,440 , of the 10,000 runs had only small gas releases (peak hydrogen concentrations less than $210 \mathrm{ppm}$ ). There were no BDGREs induced at all in 1,356 of the runs, so the hydrogen concentration remained below the initial steady-state value, which was approximately 23 ppm for the input hydrogen generation and ventilation rates.

The distribution of the fraction of retained gas released from the sediment layer for the AN-105 base case is shown in Figure 3.14. The full supernate decanting process releases a relative small fraction of the total retained gas volume. The median release fraction is 0.09 , while the 95th percentile value is 0.22 . The relatively high frequency of 0.136 at zero gas release corresponds to the 1,356 runs with no BDGREs. The largest spontaneous BDGRE in AN-105 released on the order of $20 \%$ of the retained gas in the sediment layer.

The headspace hydrogen concentration transient for a typical simulation is plotted in Figure 3.15. This run reached a peak hydrogen concentration of about $6,500 \mathrm{ppm}$. The figure shows results of five BDGREs triggered during the decant. The first large GRE, composed of one gob, began at about 10 hours, and a small single-gob release occurred at 26 hours. The second large GRE began at 30 hours and involved three more gobs. No BDGREs were induced in the last 20 hours of the decant.

These results show that decanting the supernate from AN-105 will be unlikely to challenge the LFL, though the peak headspace hydrogen concentration approaches $25 \%$ of the LFL (nearly $10,000 \mathrm{ppm}$ in AN-105) at the 95\% confidence level. The highest hydrogen concentrations are most likely to occur in the first 20 hours of decant, when the headspace is least and the hydrostatic pressure is highest. The decant process is expected to release less than a quarter of the gas. 


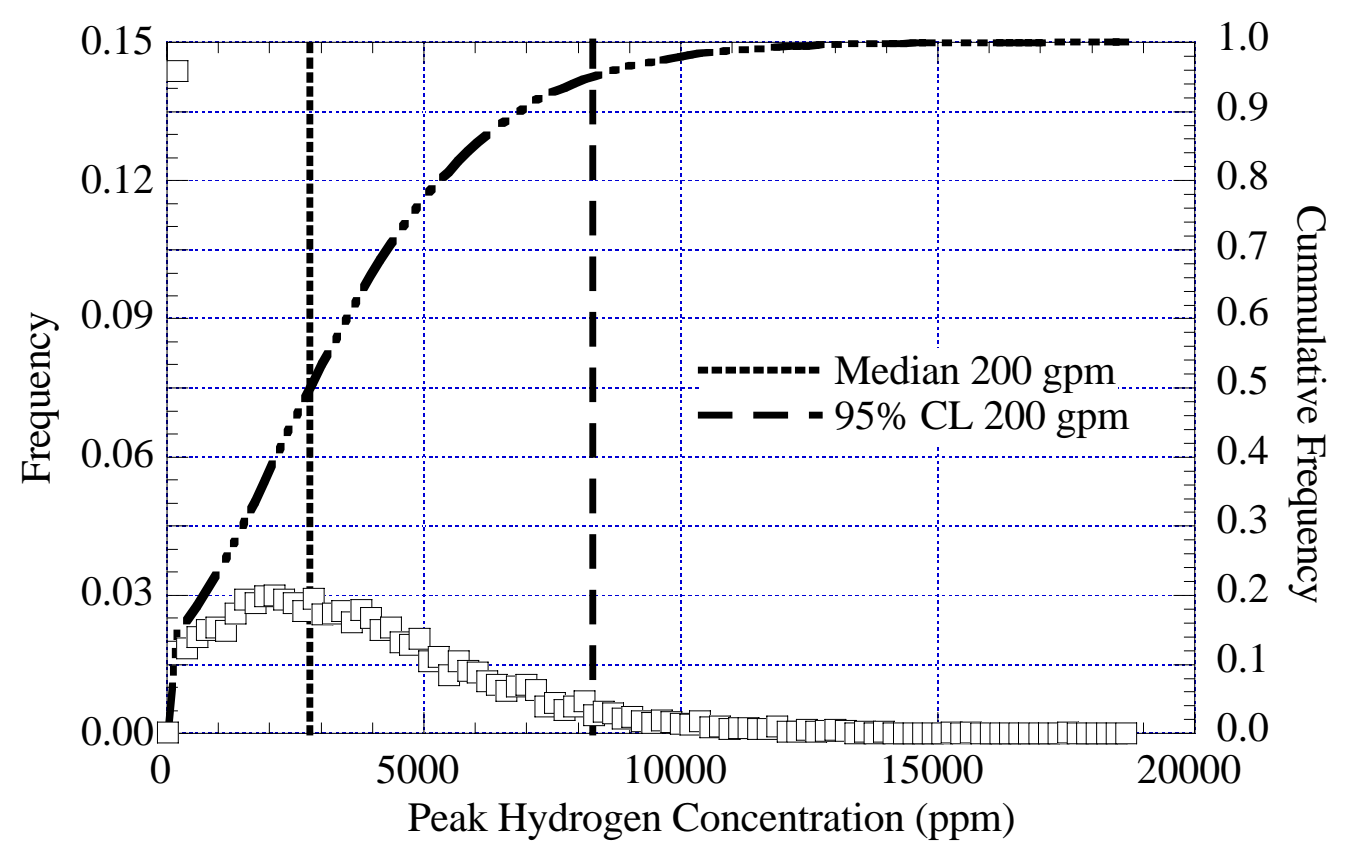

Figure 3.13. Peak Hydrogen Concentration for the AN-105 Base Case

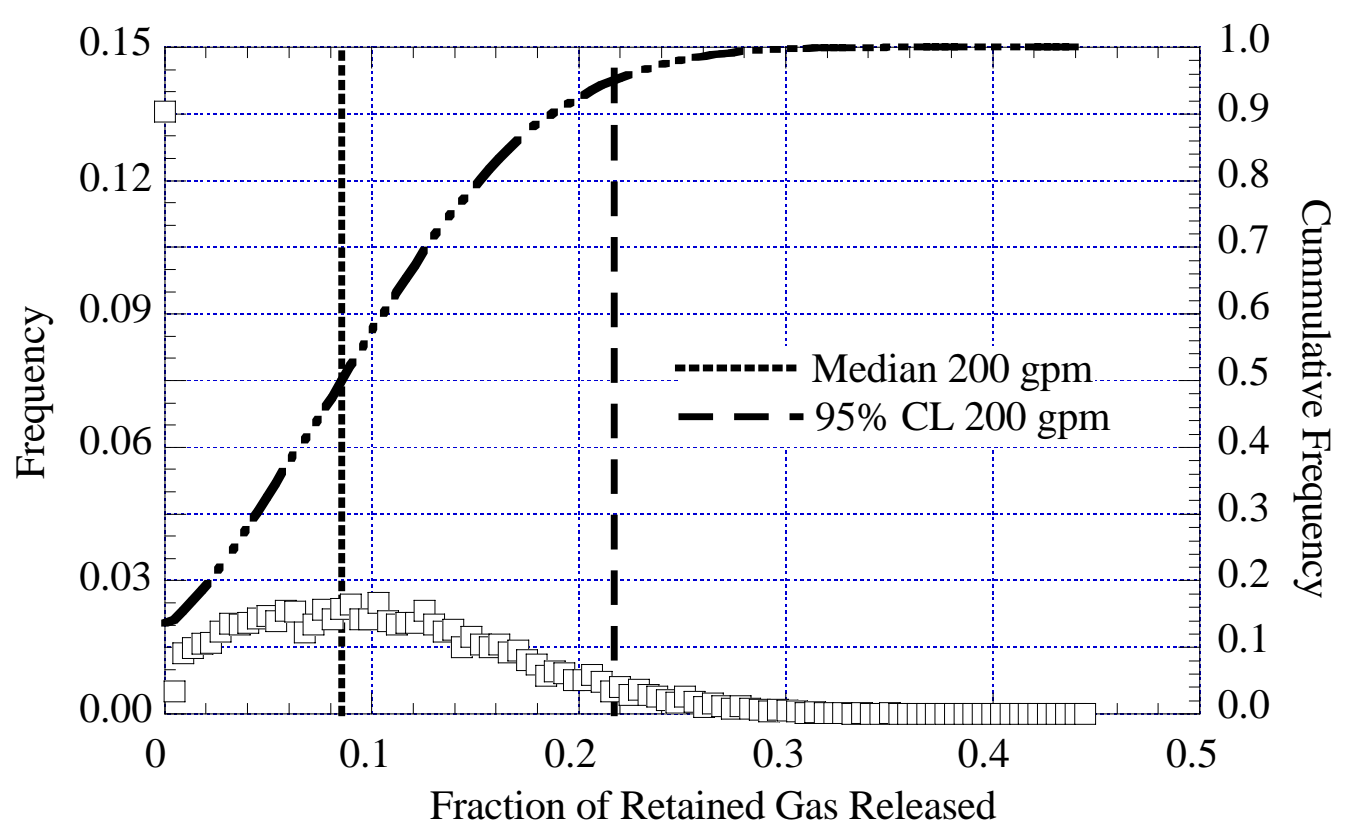

Figure 3.14. Gas Release Fraction for the AN-105 Base Case 


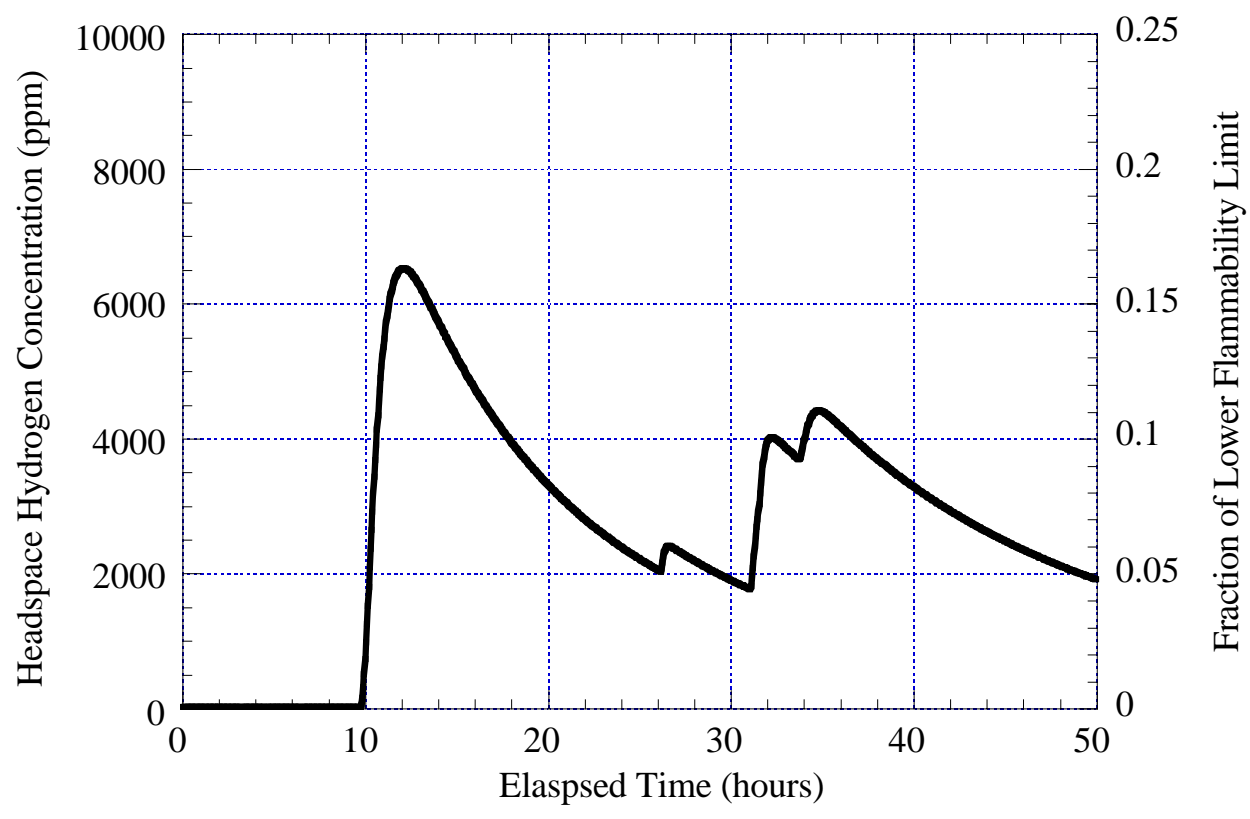

Figure 3.15. Representative Hydrogen Concentration History for the AN-105 Base Case

Because the gas releases are rapid as compared to the ventilation rate (see Section 3.2), the maximum hydrogen concentration results are relatively insensitive to changes in the ventilation rate. Varying the ventilation rate by a factor of two alters the maximum hydrogen concentration at the $95 \%$ confidence level by approximately $10 \%$.

\subsubsection{Effect of Decant Rate}

The decant flow rate is the only parameter over which the operators have direct control. It would be beneficial if a lower decant rate would reduce the peak hydrogen concentration by increasing the time between induced BDGREs. A case was run at a decant rate of $30 \mathrm{gpm}$, less than $1 / 6$ of that used in the base case, to investigate this. The 30-gpm rate was chosen as the minimum rate practical for future operations.

Figure 3.16 shows the distribution of peak hydrogen concentrations for the 30 -gpm decant rate. The median and 95th percentile value for the base case are also shown. While the lower decant rate lowered the peak hydrogen concentrations measurably, the difference was not significant. The median value decreased from $2,800 \mathrm{ppm}$ in the base case to 2,600 ppm and the 95th percentile value from $8,300 \mathrm{ppm}$ to $8,000 \mathrm{ppm}$. The number of runs in which no BDGREs occurred (1356) remained the same as in the base case.

The distribution of the fraction of retained gas released (Figure 3.17) is virtually identical to the base case, showing that a given reduction in hydrostatic pressure releases the same fraction of retained gas regardless of the rate at which it is reduced (assuming suspended solids do not settle out). This also will be shown for the stop-start control strategy. The main consequence of the lower decant rate is to lengthen the time required for decant from 50 hours to almost two weeks. 


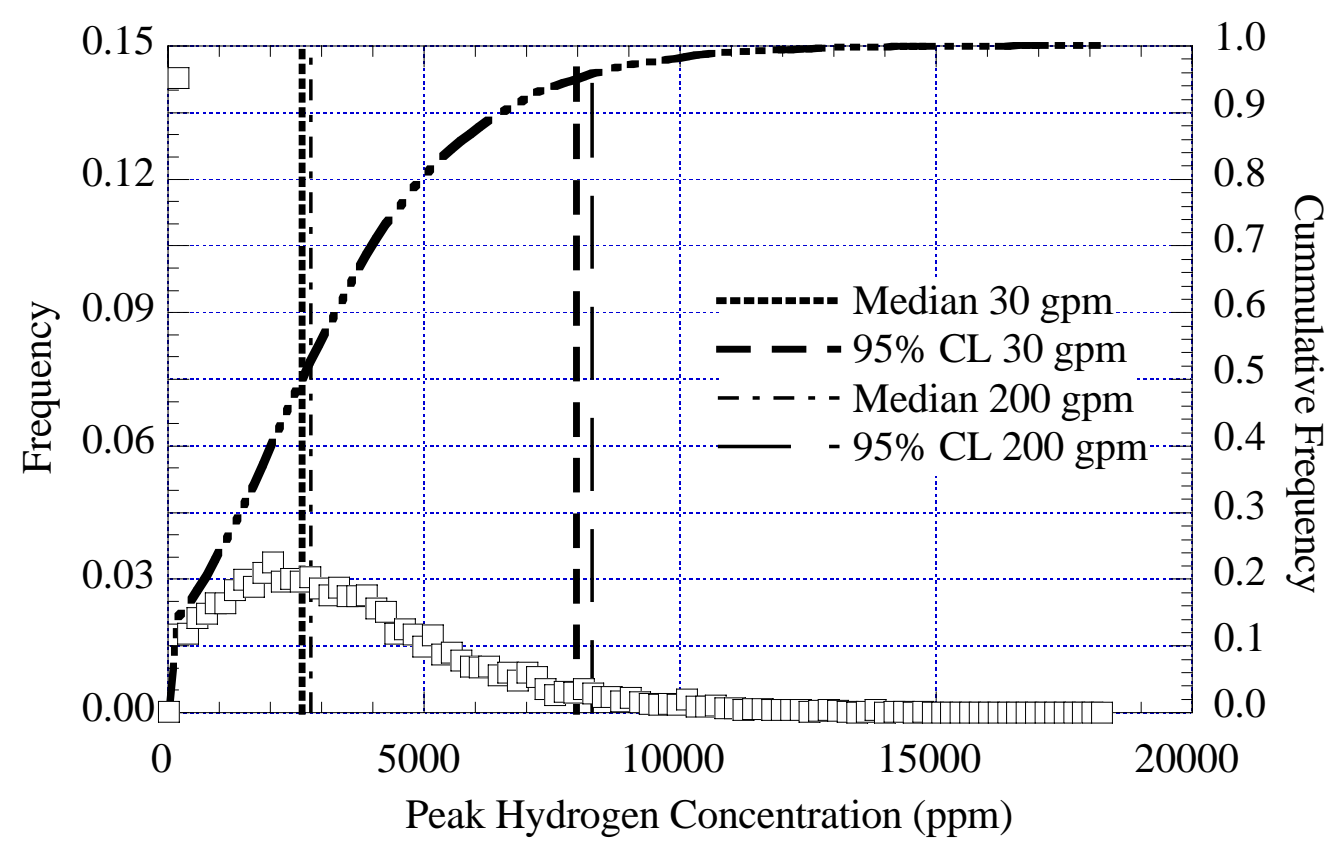

Figure 3.16. Hydrogen Concentration for 30 gpm Decant Rate in AN-105

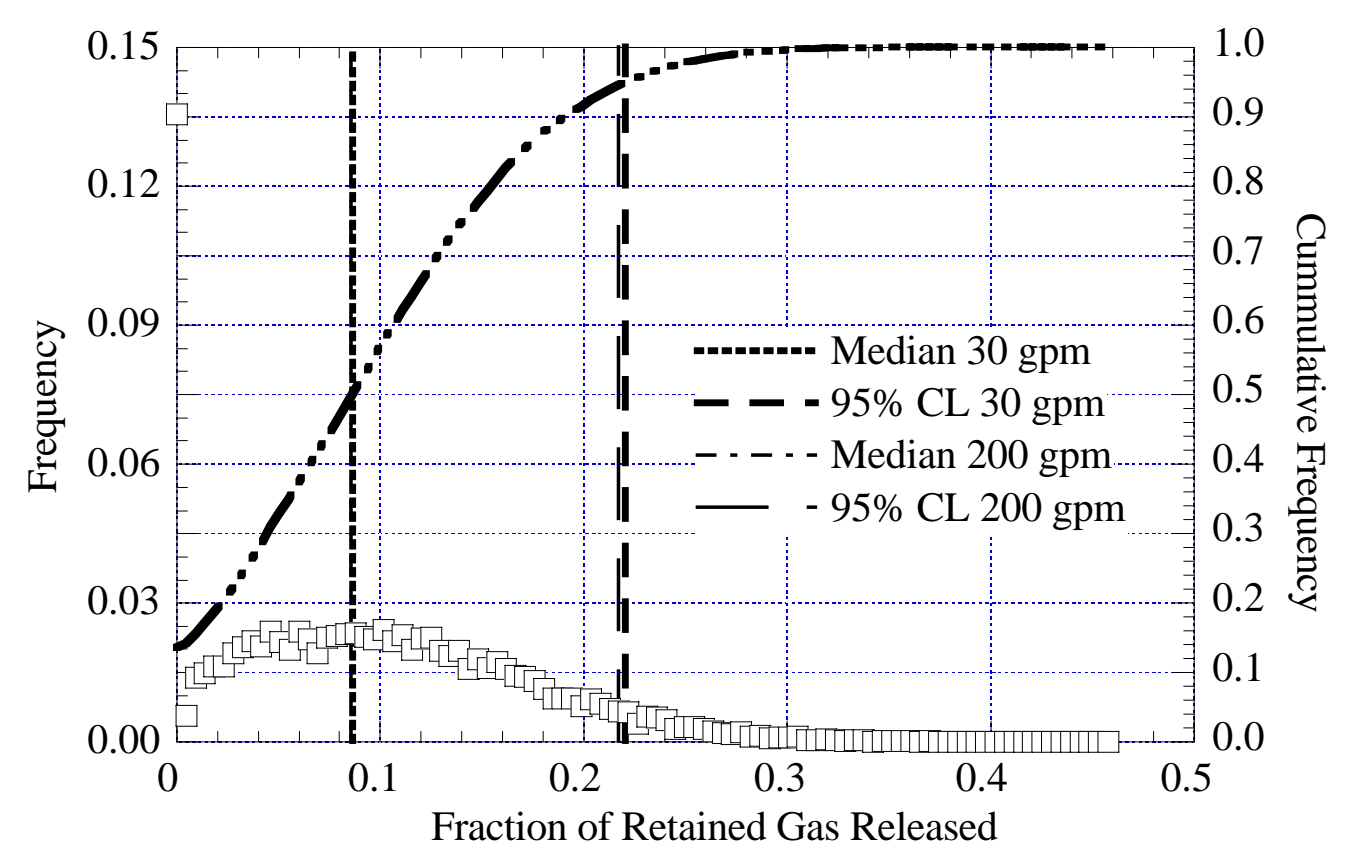

Figure 3.17. Fraction of Gas Released for 30 gpm Decant Rate in AN-105 


\subsubsection{Stop-Start Control Strategy}

Tank farm operations are required to be halted if the gas monitoring instruments measure a hydrogen concentration exceeding 6,250 ppm in the headspace (CHG 2000). This control was set to prevent the concentration from reaching the LFL on the assumption that the operation was causing the gas release. Operations can theoretically resume when the hydrogen concentration drops back below 6,250 ppm; however, a lower restart threshold is usually used. The SY-101 mixer pump safety assessment (Sullivan 1995) specified 500-ppm for restart.

To check the effect of a stop-start control strategy, the base case was rerun with the decant rate set to zero at hydrogen concentrations above $6,250 \mathrm{ppm}$ and restarted at $500 \mathrm{ppm}$. The resulting peak hydrogen concentration distribution is shown in Figure 3.18. The indicators for the base case are also shown. The stop-start control strategy had less effect on the peak hydrogen concentration than reducing the decant rate. The median of $2,800 \mathrm{ppm}$ is identical to the base case, and the 95th percentile value is lowered only slightly to 8,200 ppm. The distribution of gas release fraction, given in Figure 3.19, and the number of runs with no BDGREs (1365) are also virtually identical to that of the base case.

As with the 30-gpm decant rate case, the main result of the start-stop control strategy was to prolong decant by about 100 hours. This is illustrated in Figure 3.20, where the hydrogen concentration transients in the start-stop case and base case are plotted for runs using the same set of input. The first GRE, composed of one gob, began at about 10 hours; decant was halted at about 11.5 hours, when the hydrogen concentration rose above $6,250 \mathrm{ppm}$. However, the BDGRE was already under way and drove the hydrogen concentration to the same peak value as the base case. Decant restarted at about 37.5 hours, and the second GRE, composed of four gobs, started about 14.5 hours later, at 52 hours.

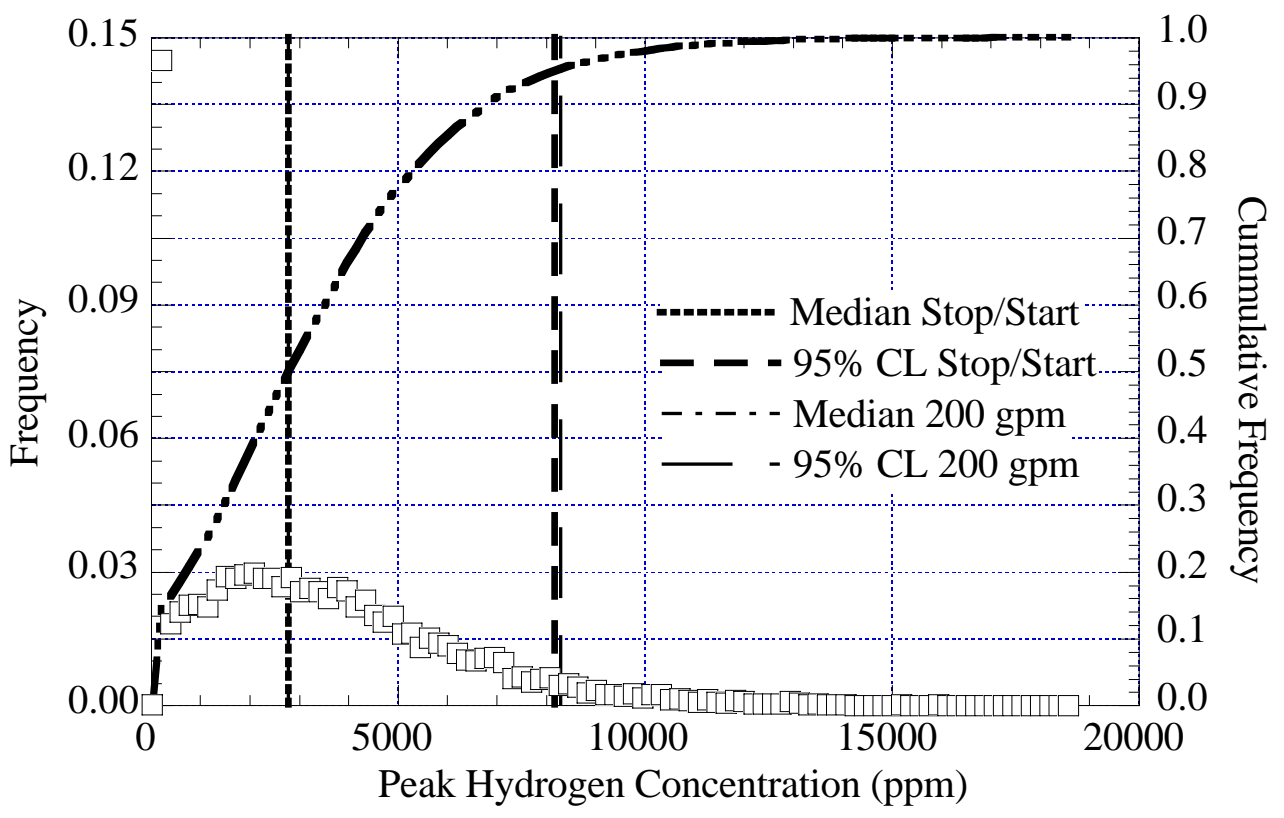

Figure 3.18. Peak Hydrogen Concentration for the Stop-Start Case in AN-105 


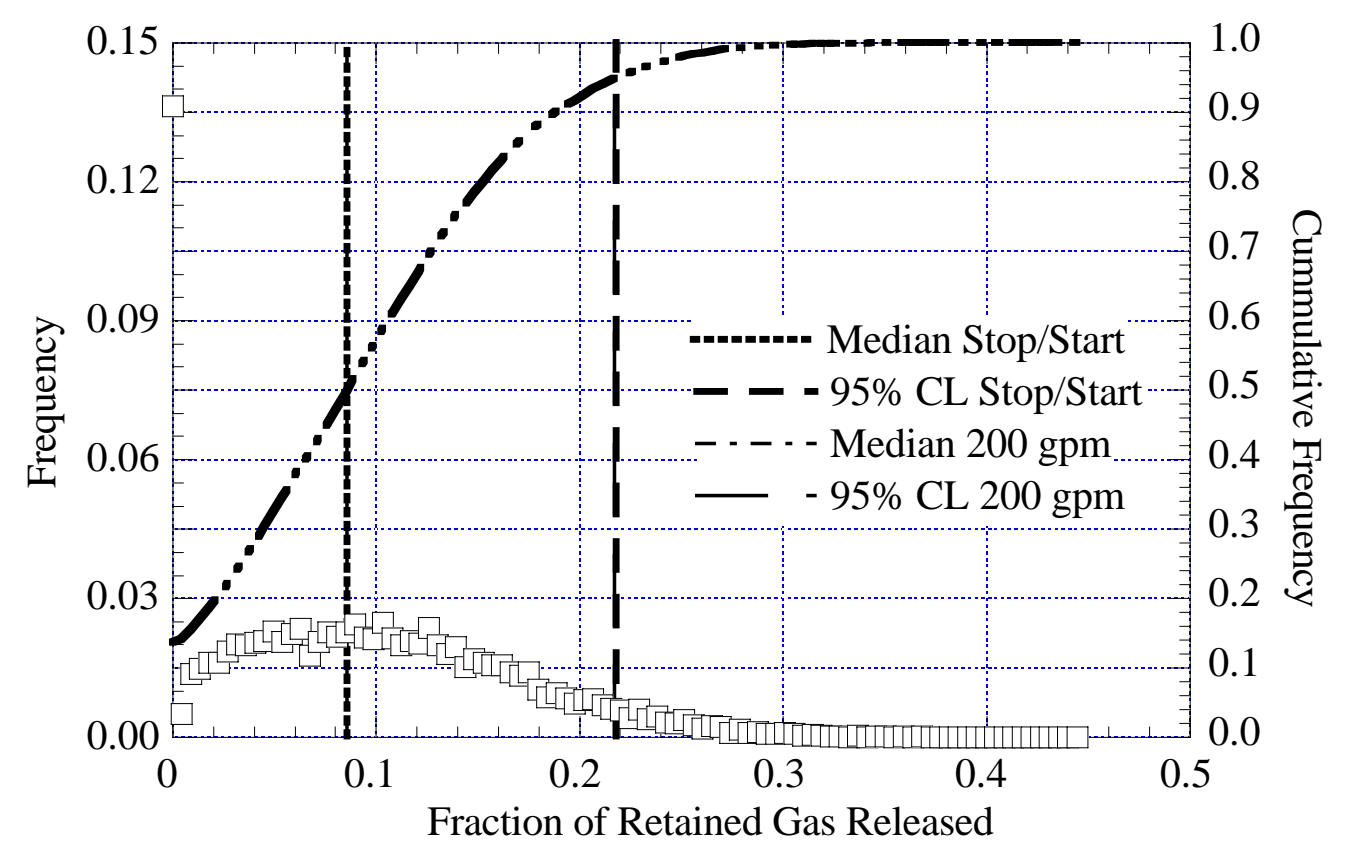

Figure 3.19. Gas Release Fraction for the Stop-Start Case in AN-105

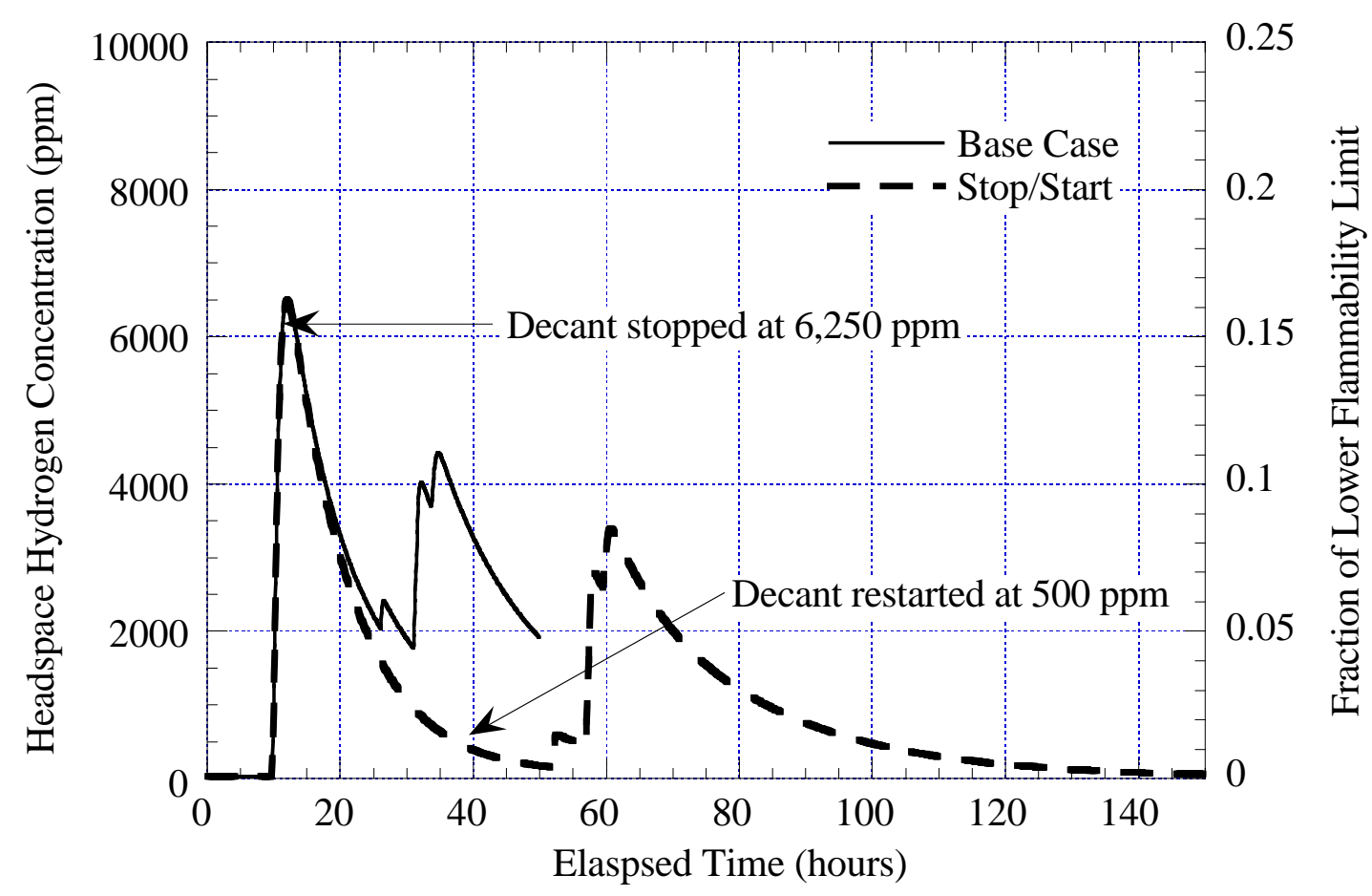

Figure 3.20. Hydrogen Concentration Transient for Stop-Start Case in AN-105 
There were 1,262 runs out of the 10,000 with hydrogen concentrations reaching 6,250 ppm, requiring 1266 stop-start periods (four runs had two stop-start periods apiece). During these periods the hydrogen concentration kept rising, reaching a maximum 2.5 hours after decant stopped. The median peak concentration during a stop-start period was about 7,600 ppm, and the 95th percentile value was almost $12,000 \mathrm{ppm}$. Because a hydrogen concentration rising past $6,250 \mathrm{ppm}$ is generally the result of a BDGRE in progress, and the gas release from gobs already at the surface is not affected by decanting, stopping the decant does not reduce the peak hydrogen concentration.

\subsubsection{Water Backfill Control Strategy}

Since decant-induced BDGREs are the result of decreasing hydrostatic pressure, it was believed that maintaining the pressure by backfilling with water might prevent or reduce the frequency of BDGREs. However, because water is much less dense than the concentrated supernate, the hydrostatic pressure cannot be maintained, and some potential for induced BDGREs remains. In AN-105, the initial hydrostatic pressure on the retained gas is about $2.0 \mathrm{~atm}$. A full supernate decant reduces the pressure to $1.3 \mathrm{~atm}$, while the minimum pressure for a water backfill is $1.8 \mathrm{~atm}$. However, a negative side effect of the backfill is that the tank headspace does not increase (except minimally due to gas release) as supernate is removed. This means that the same gas release results in a higher hydrogen concentration. This is exacerbated by the higher gas release fraction coincident with the higher hydrostatic pressure.

The results of these combined effects are illustrated in the distribution of peak hydrogen concentration shown in Figure 3.21. The most striking effect of the water backfill is that the median peak hydrogen concentration is at the steady-state background concentration of $23 \mathrm{ppm}$. This is the direct result of there being no BDGREs in 7,653 of the 10,000 runs. However, due to the smaller headspace, the peak hydrogen concentration at the 95 th percentile value, 7,600 ppm, was essentially the same as that of the base case. The fraction of retained gas released is also much lower than the base case, as shown in Figure 3.22. The median is 0 and the 95th percentile value 0.13 - base case is 0.22 . This means that many fewer BDGREs occurred in all runs.

The behavior of induced BDGREs during water backfill is presented more clearly in the hydrogen concentration transient in Figure 3.23. Plots for the same set of input values in the base case and stop-start case are also shown. The water backfill plot shows that the first BDGRE occurs about 20 hours later than in the other cases but cause a higher hydrogen concentration because of the smaller headspace. The remaining four BDGREs (base case run has five BDGREs total) are not triggered. These results show that, while water backfill does not reduce the bounding hydrogen concentration, it does very significantly reduce the probability of BDGREs and the total volume of gas released. 


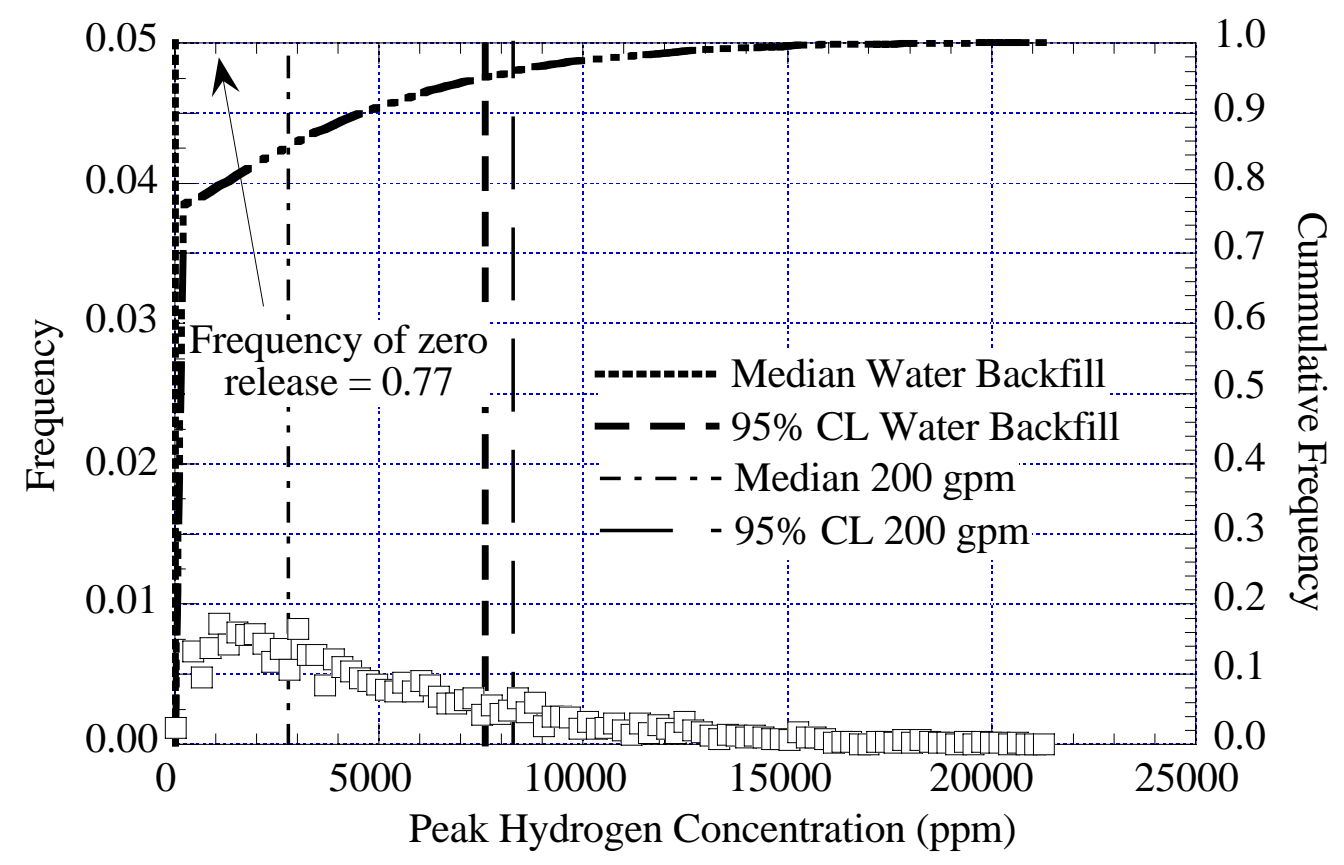

Figure 3.21. Peak Hydrogen Concentration for Water Backfill in AN-105

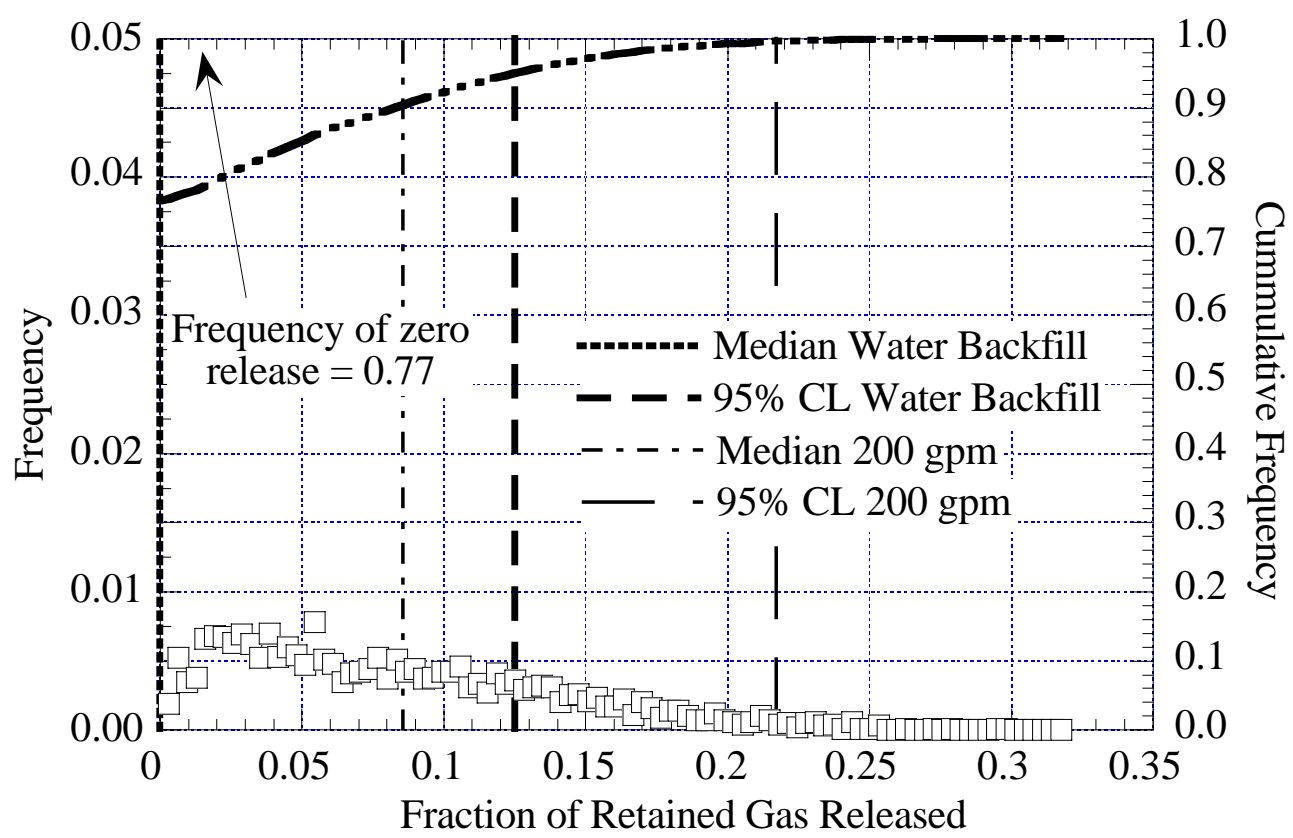

Figure 3.22. Fraction of Gas Released for Water Backfill in AN-105 


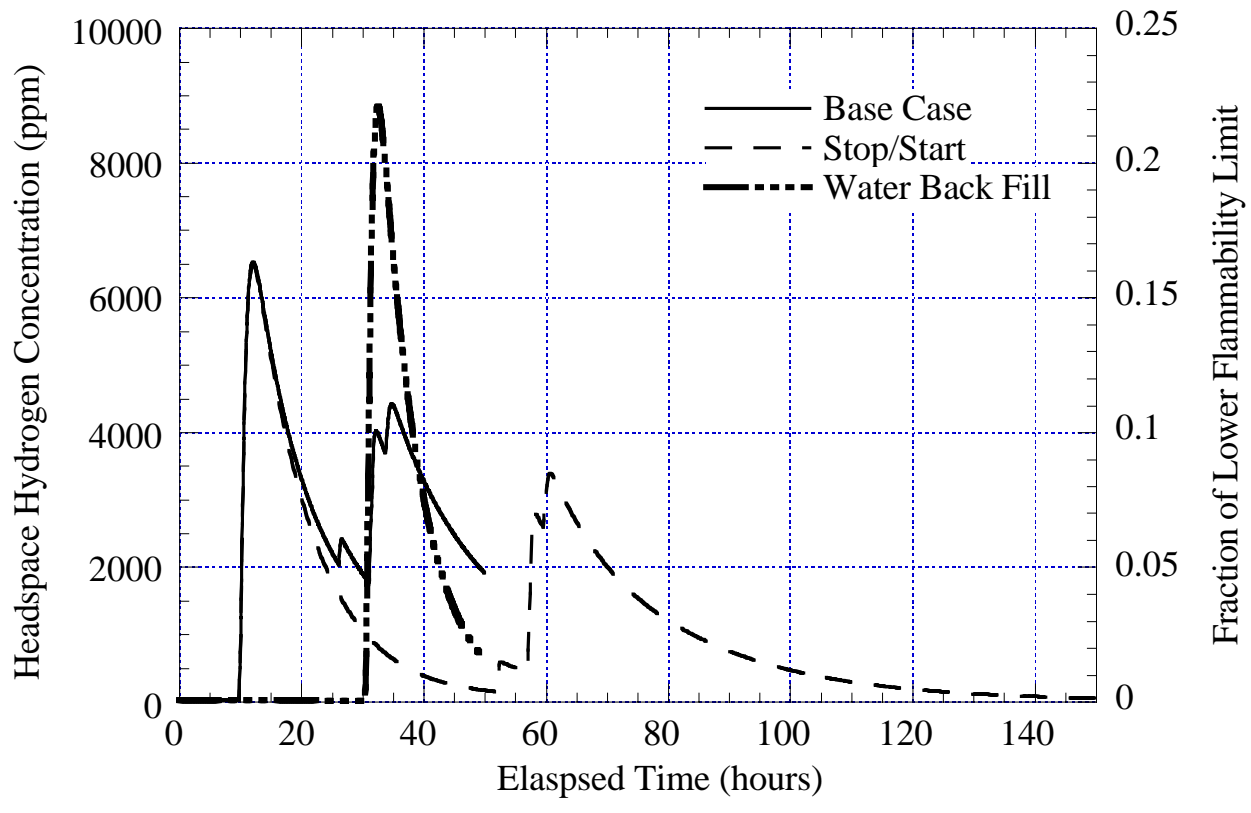

Figure 3.23. Hydrogen Concentration Transient for Water Backfill in AN-105

\subsubsection{Summary of Analyses for AN-105}

Gas releases induced by supernate decant in AN-105 are very unlikely to approach flammability. The median value of the peak hydrogen concentration during decant was about $3,000 \mathrm{ppm}$, and about $8,000 \mathrm{ppm}$ at the $95 \%$ confidence level. It is relatively insensitive to decant rate and was not reduced by stopping the decant at the action level of 6,250 ppm. Backfilling with water significantly reduced the median peak hydrogen concentration, though it did not reduce the bounding value of peak hydrogen concentration. It also greatly reduced the probability of BDGREs. More than $75 \%$ of the water backfill runs did not have a BDGRE. The fraction of total gas released during decant was virtually identical in the base case, the reduced decant rate, and the stop-start control strategy case at $22 \%$. Only the water backfill reduced it significantly. These results are summarized in Table 3.7.

Table 3.7. Summary of Decant Analysis Results in AN-105

\begin{tabular}{||l|l|c|c||}
\hline Run & Quantity & Median & 95\% CL \\
\hline Base Case & Hydrogen $(\mathrm{ppm})$ & 2,800 & 8,300 \\
\hline & Fraction Gas Release & 0.09 & 0.22 \\
\hline 30 gpm Decant & Hydrogen (ppm) & 2,600 & 8,000 \\
\hline & Fraction Gas Release & 0.09 & 0.22 \\
\hline Stop-Start Control & Hydrogen (ppm) & 2,800 & 8,200 \\
\hline Water Backfill & Fraction Gas Release & 0.09 & 0.22 \\
\hline & Hydrogen (ppm) & 23 & 7,600 \\
\hline & Fraction Gas Release & 0.00 & 0.13 \\
\hline
\end{tabular}




\subsubsection{Sensitivity Analysis}

A sensitivity analysis was conducted on the AN-105 decant results to determine which variables have the most impact on the output results. The results of only the base case are shown because analysis results for the other cases were proven very similar.

The set of inputs and outputs is assumed to be a multivariate normal distribution. A multivariate analysis of variance (MANOVA) was performed on the data. The analysis compares the matrix of hydrogen concentration outputs (1,000 results per simulation) to the set of inputs. The error associated with the model is assumed to be distributed multivariate normal with mean zero and variance matrix $\Sigma$. It is assumed that no significant interactions exist between the independent variables in the data. The results are shown in Table 3.8. Low p-values $(<0.05)$ indicate highly significant impact on the results. In the scheme of the decant model, gob 1 has the highest initial average gas fraction, gob 2 has the second highest average gas fraction, and so on.

No unexpected results are achieved. The model results are most sensitive to the liquid density, sediment layer density, and the gas fraction and diameter of gob 1. The layer densities dictate the gas fraction at which a gob may become buoyant (also affected by sediment yield stress), and the liquid density affects the pressure change for a volume of supernate removed and amount of gas released in a BDGRE. The gas fraction in gob $^{\circ} 1$, the highest gas fraction in the gobs, directly affects the likelihood of a BDGRE occurring during decant, the diameter of this gob directly affects the quantity of gas released, and the hydrogen concentration in the retained gas dictates the volume of hydrogen in the headspace. The gas fraction profile in the gob dictates the rate at which the gas fraction changes with decant and thus directly impacts the

Table 3.8. Sensitivity Results from Base Case

\begin{tabular}{||l|r||}
\hline \multicolumn{1}{|c|}{ Parameter } & P-Value \\
\hline Crust thickness & $<0.0001$ \\
\hline Sediment yield stress & $<0.0001$ \\
\hline Liquid density & 0.0000 \\
\hline Sediment layer density & 0.0000 \\
\hline Hydrogen concentration in retained gas & $<0.0001$ \\
\hline Fraction of gob material suspended into supernate & $<0.0001$ \\
\hline Time to peak release rate, gob 1 & $<0.0001$ \\
\hline Time to peak release rate, gob 2 & $<0.0001$ \\
\hline Time to peak release rate, gob 3 & 0.0009 \\
\hline Gob gas fraction, gob 1 & 0.0000 \\
\hline Gob gas fraction, gob 2 & $<0.0001$ \\
\hline Gob gas fraction, gob 3 & 0.0091 \\
\hline Gob diameter, gob 1 & 0.0000 \\
\hline Gob diameter, gob 2 & $<0.0001$ \\
\hline Gob gas fraction profile, gob 1 & $<0.0001$ \\
\hline Gob gas fraction profile, gob 2 & $<0.0001$ \\
\hline Gob gas fraction profile, gob 3 & 0.0468 \\
\hline Gob gas fraction profile, gob 4 & 0.0085 \\
\hline
\end{tabular}


results. The time to peak release rate affects the hydrogen concentration and stimulates subsequent BDGREs in conjunction with the fraction of the gob material suspended in the supernate.

\subsection{Supernate Decant in AN-103, AN-104, and AW-101}

Decant-induced gas releases in the three other tanks were also analyzed. Four cases were run to investigate the sensitivity of the results to the decant rate and the effects of the two postulated control strategies. The initial conditions for each tank were determined from historical data and measurements the same way as AN-105 (see Section 3.2). Both 5,000 and 10,000 simulations were conducted for the base case in each tank. Because the results of these simulations were equivalent, only 5,000 simulations were conducted to investigate the effects of varying the decant rate and of the control strategies in an effort to control computational time. The favorable comparison of the base case runs indicates that the validity of the results is not compromised by the decrease in run count. The decant results for all of the tanks are summarized in Section 3.5.

\subsubsection{AN-103 Decant Results}

Tank AN-103 contains by far the highest retained gas volume of the four tanks considered in this study. It also has exhibited the fewest and smallest spontaneous BDGREs, so there are relatively few good observations from which to input parameter distributions. By all indications (i.e., direct void measurements, barometric pressure response, surface level rise, and lack of large GREs), the entire waste volume is very near neutral buoyancy. This required a distribution for the gob gas fraction that was skewed toward the neutral buoyant gas fraction (see Section 3.2.3) to satisfy the constraint on total gas volume.

Because a high percentage of the gobs in the tank are initially very close to neutral buoyancy, BDGREs occur relatively early in every run (only $2 \%$ of the runs had no BDGREs occur). This results in rather high hydrogen concentration results. The peak hydrogen concentration and the gas release fraction results for the base case are shown in Figures 3.24 and 3.25. The median of the peak hydrogen concentration is $10,500 \mathrm{ppm}$, and the 95 th percentile value is $21,100 \mathrm{ppm}$, or about $50 \%$ of the LFL. None of the runs exceeded the LFL. The median and 95th percentile values of the gas release fraction are 0.18 and 0.33 , respectively.

The results for the $30 \mathrm{gpm}$ decant are similar to those of the base case (Figures 3.26 and 3.27. The stop-start control results are shown in Figures 3.28 and 3.29. Almost $80 \%$ of the runs had hydrogen concentrations exceed 6,250 ppm, requiring 4,811 stop-start periods. About $1 \%$ of the runs required three stop-start periods (i.e., 6,250 ppm was exceeded three times during the decant for $1 \%$ of the simulations) and over $20 \%$ required at least two stop-start periods. The hydrogen concentration during these stopped periods kept rising, reaching a maximum approximately 3.5 hours after decant stopped. The median peak concentration during a stop-start period was about 9,400 ppm, and the 95th percentile value was almost 50\% of the LFL at 19,400 ppm.

The water backfill control results are shown in Figures 3.30 and 3.31. As with AN-105, the control strategies do not significantly alter the $95^{\text {th }}$ percentile results, and median results for the water backfill case are notably lower. Only $45 \%$ of the water backfill runs had no BDRGEs. 


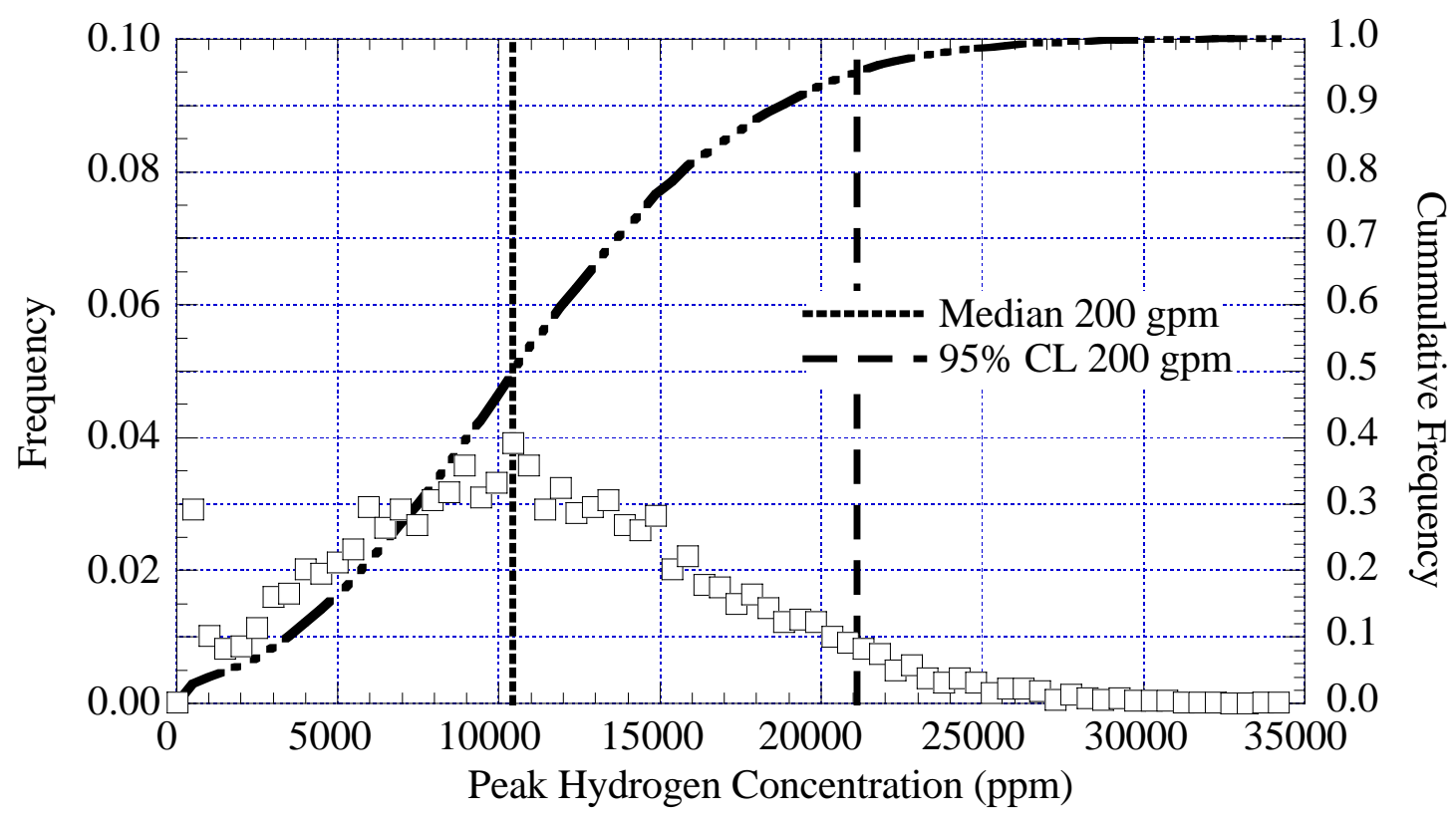

Figure 3.24. Peak Hydrogen Concentration for the AN-103 Base Case

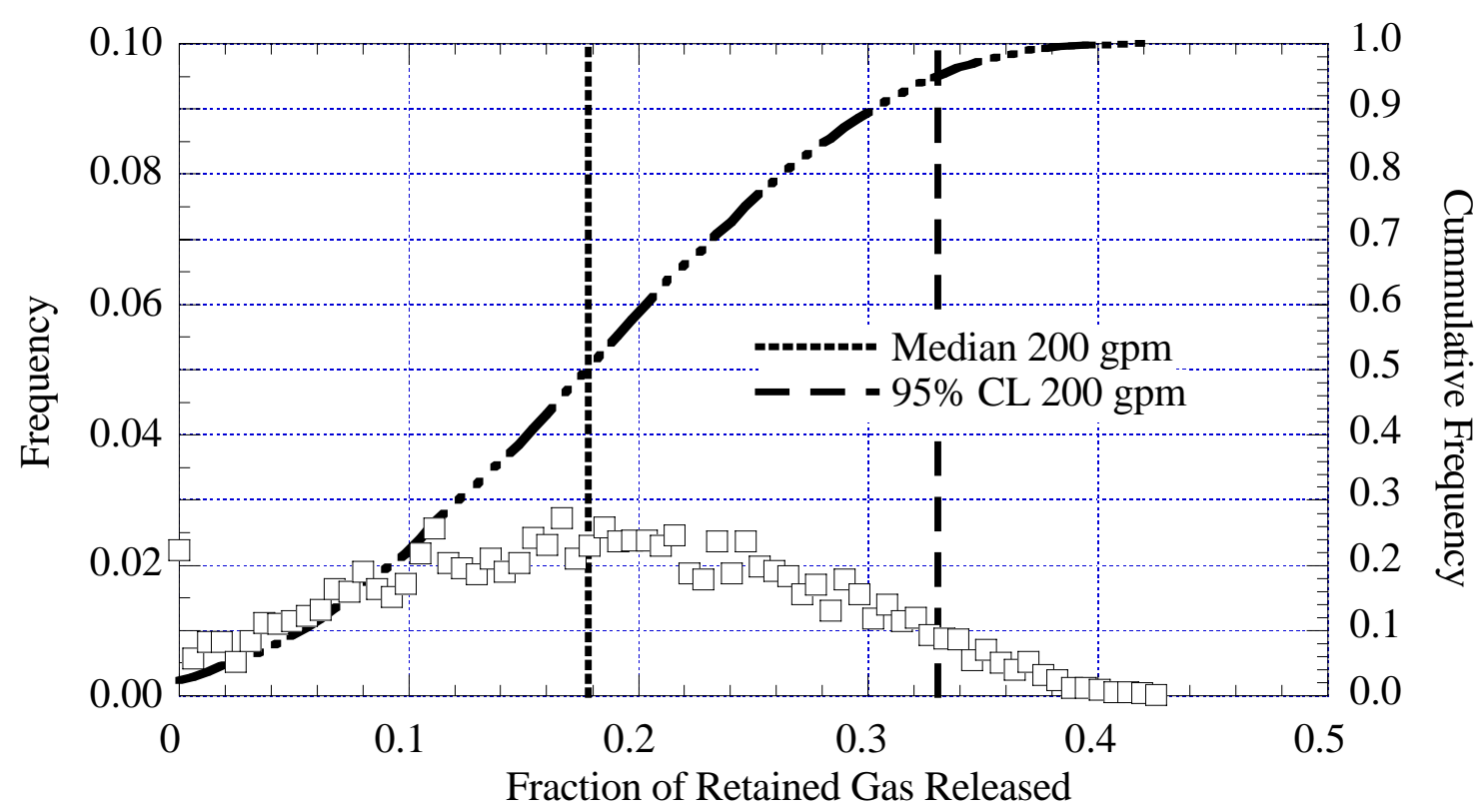

Figure 3.25. Gas Release Fraction for the AN-103 Base Case 


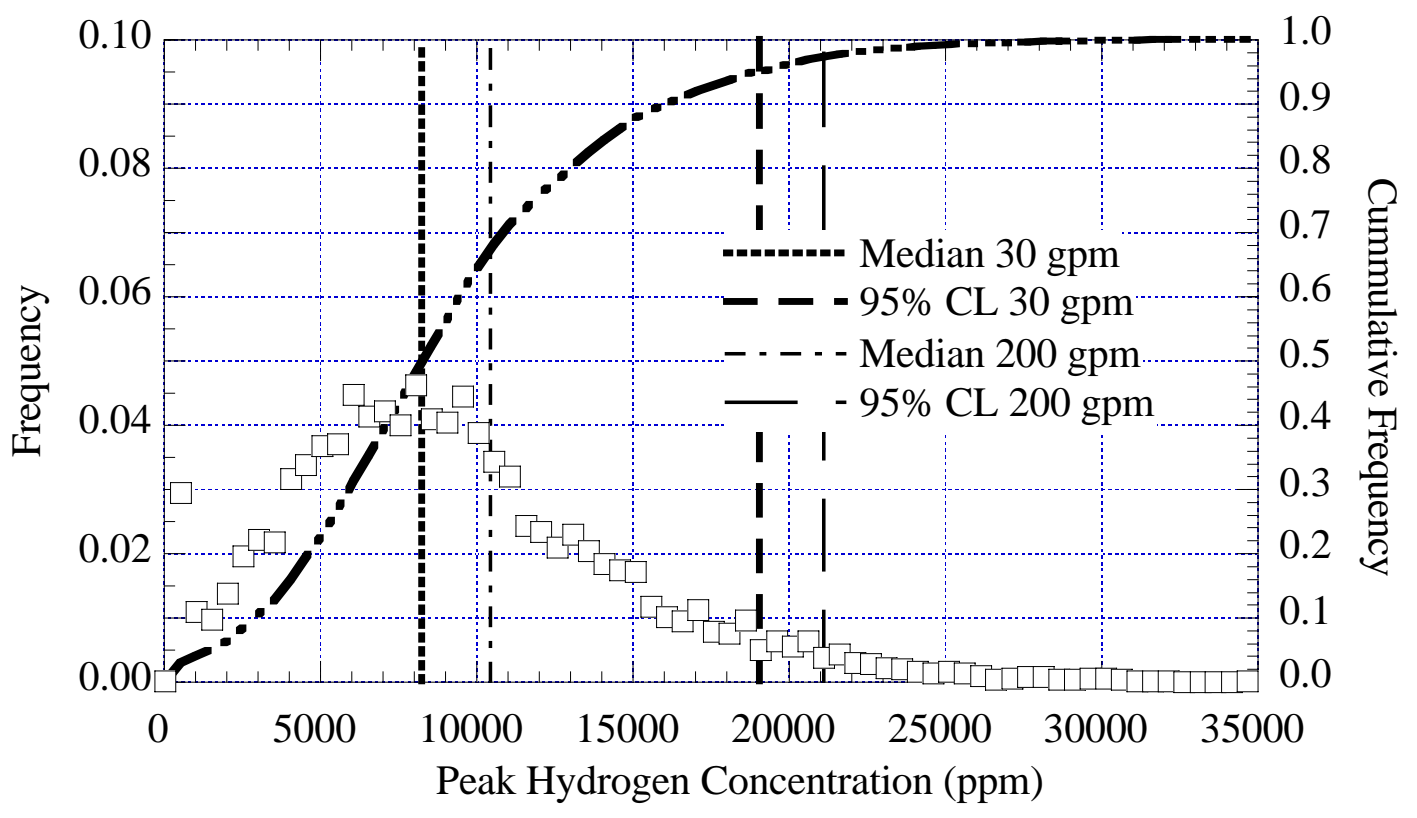

Figure 3.26. Peak Hydrogen Concentration for 30 gpm Decant Rate in AN-103

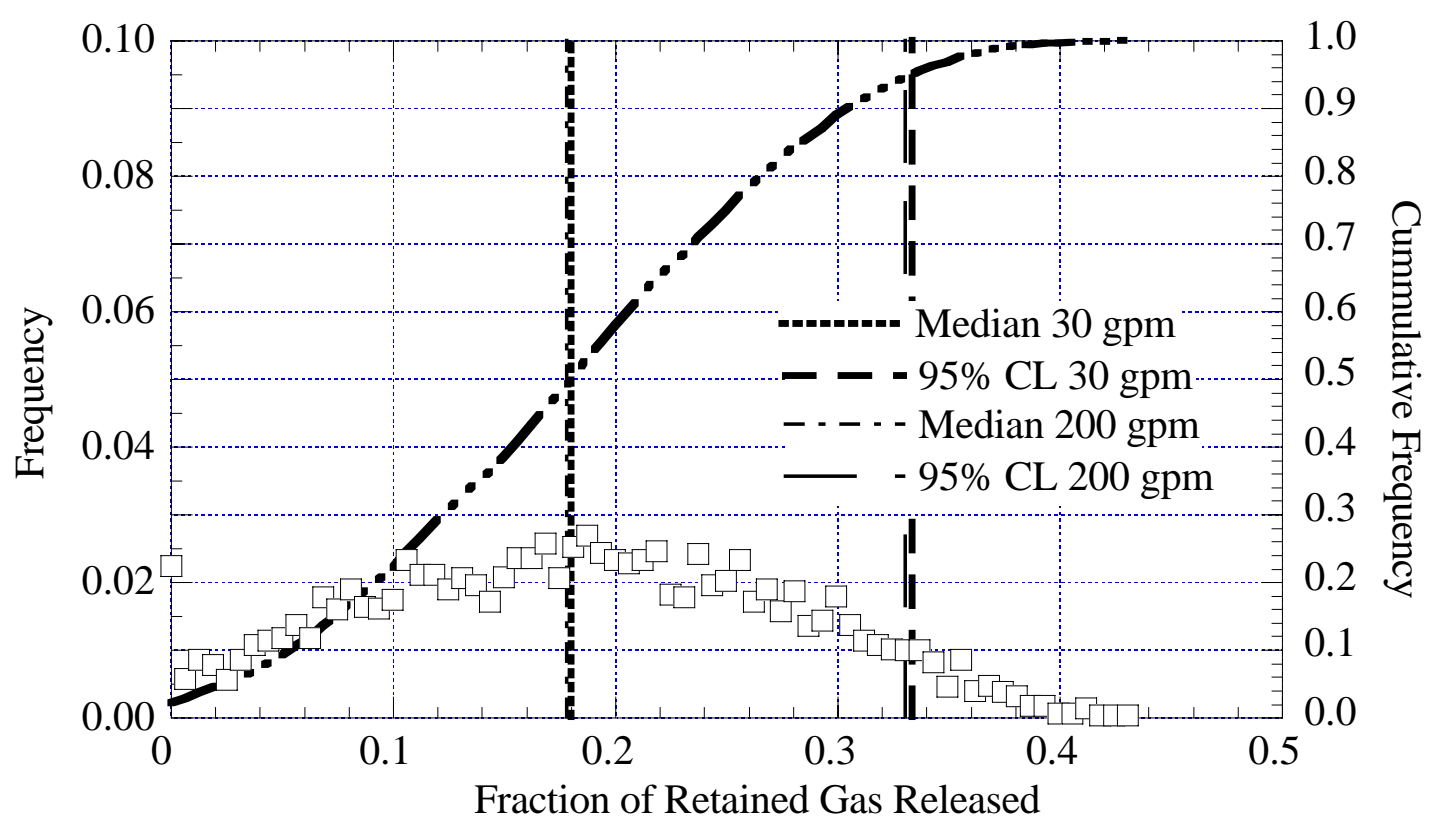

Figure 3.27. Gas Release Fraction for 30 gpm Decant Rate in AN-103 


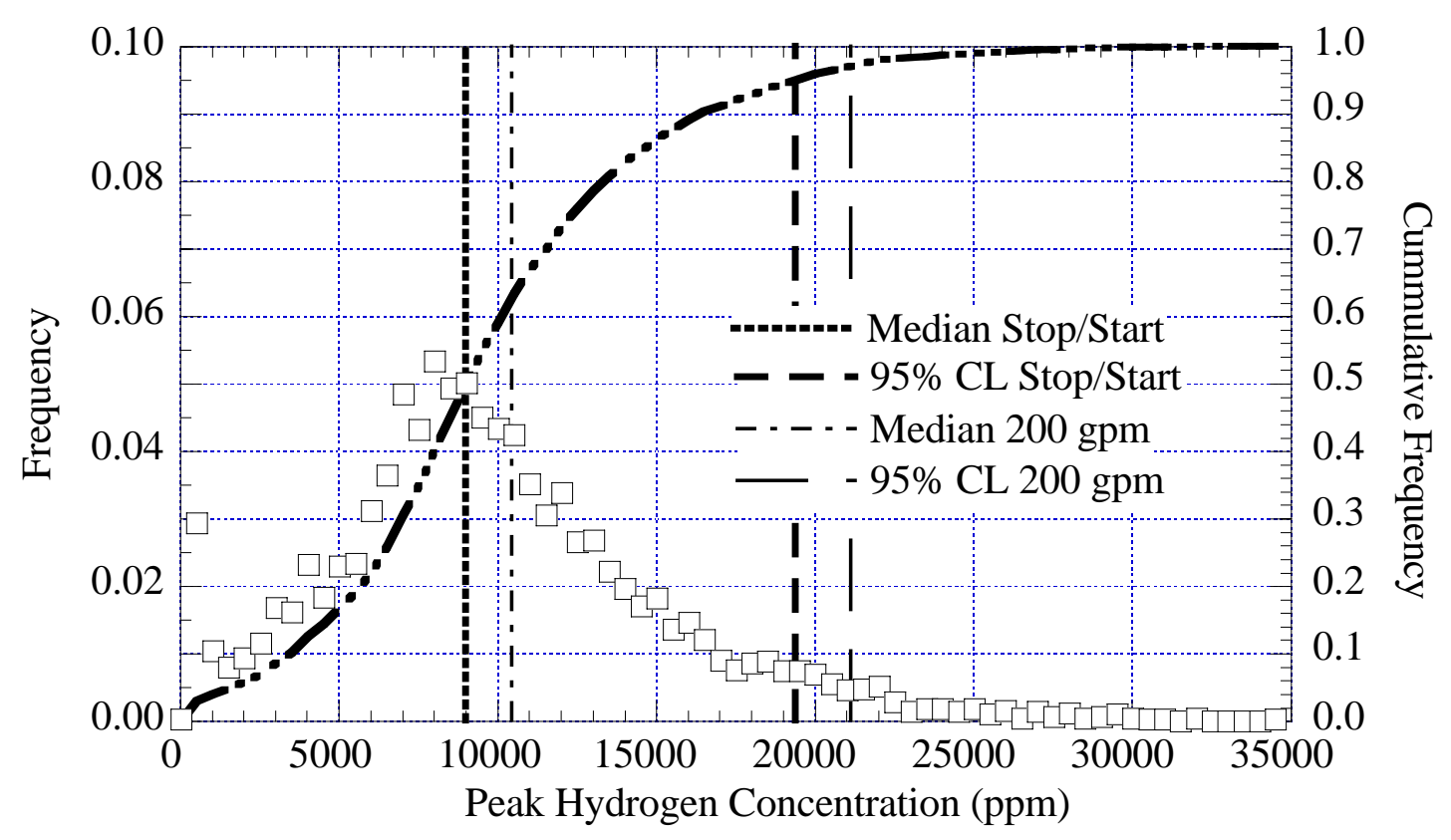

Figure 3.28. Peak Hydrogen Concentration for the Stop-Start Case in AN-103

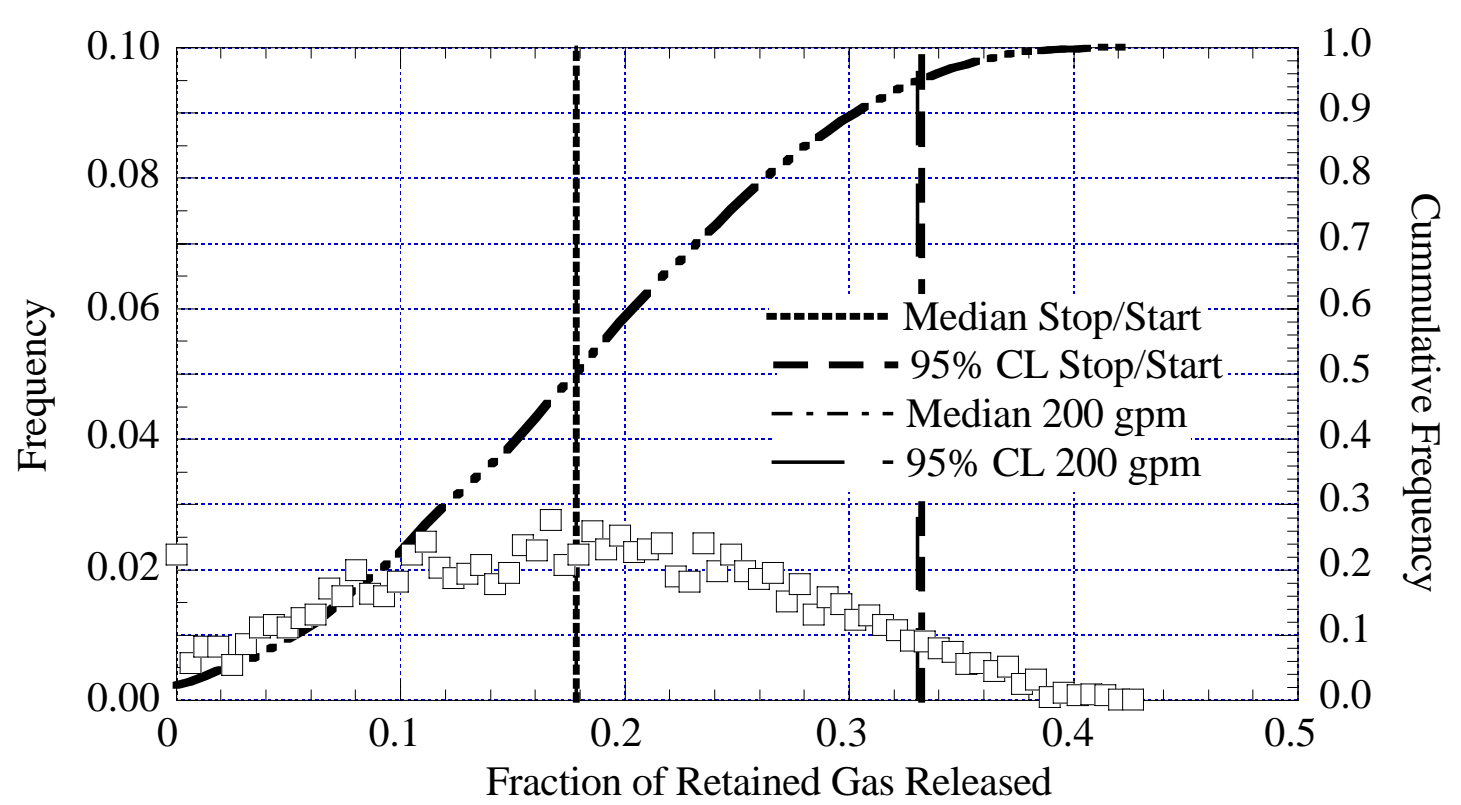

Figure 3.29. Gas Release Fraction for the Stop-Start Case in AN-103 


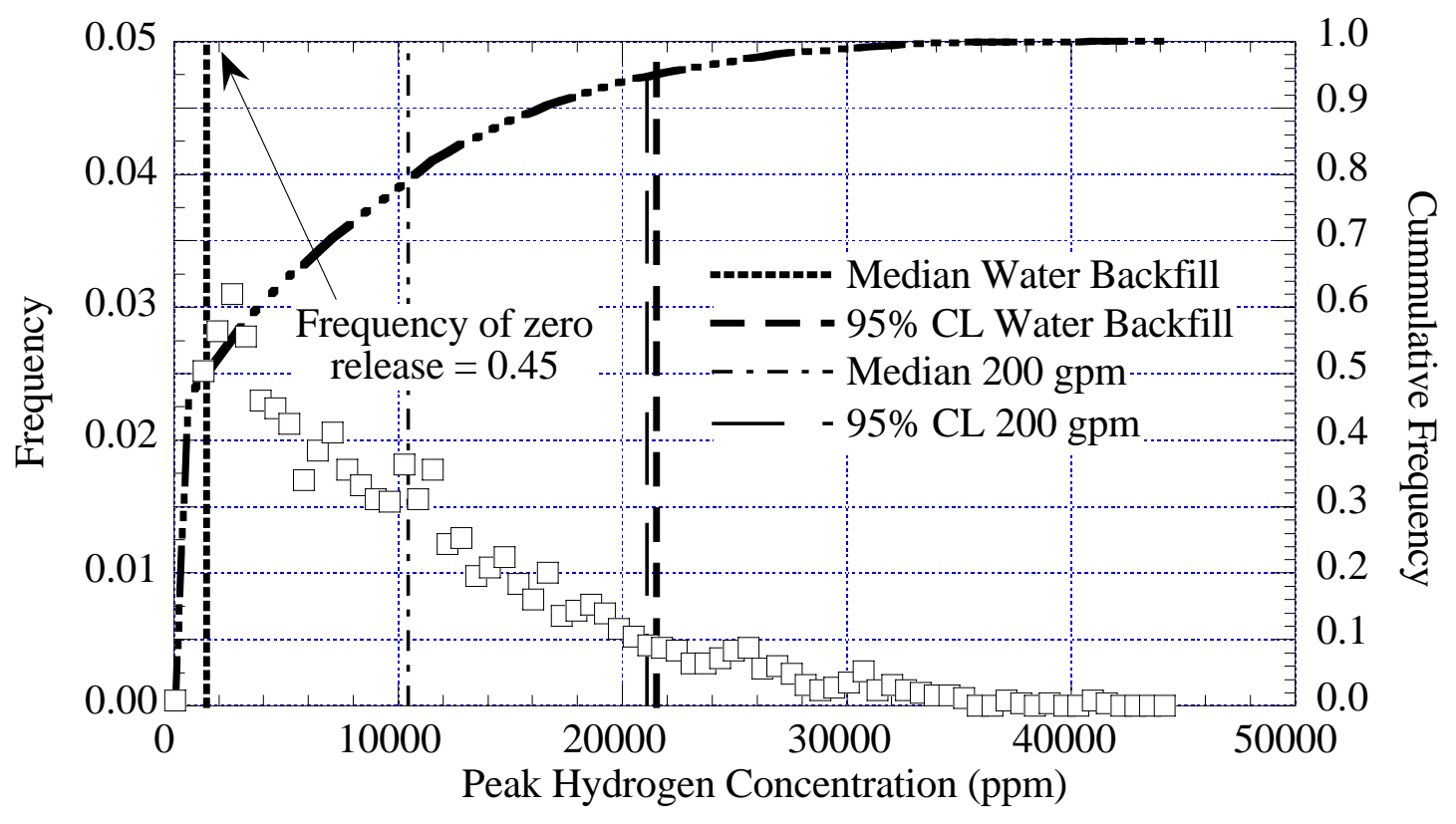

Figure 3.30. Peak Hydrogen Concentration for Water Backfill in AN-103

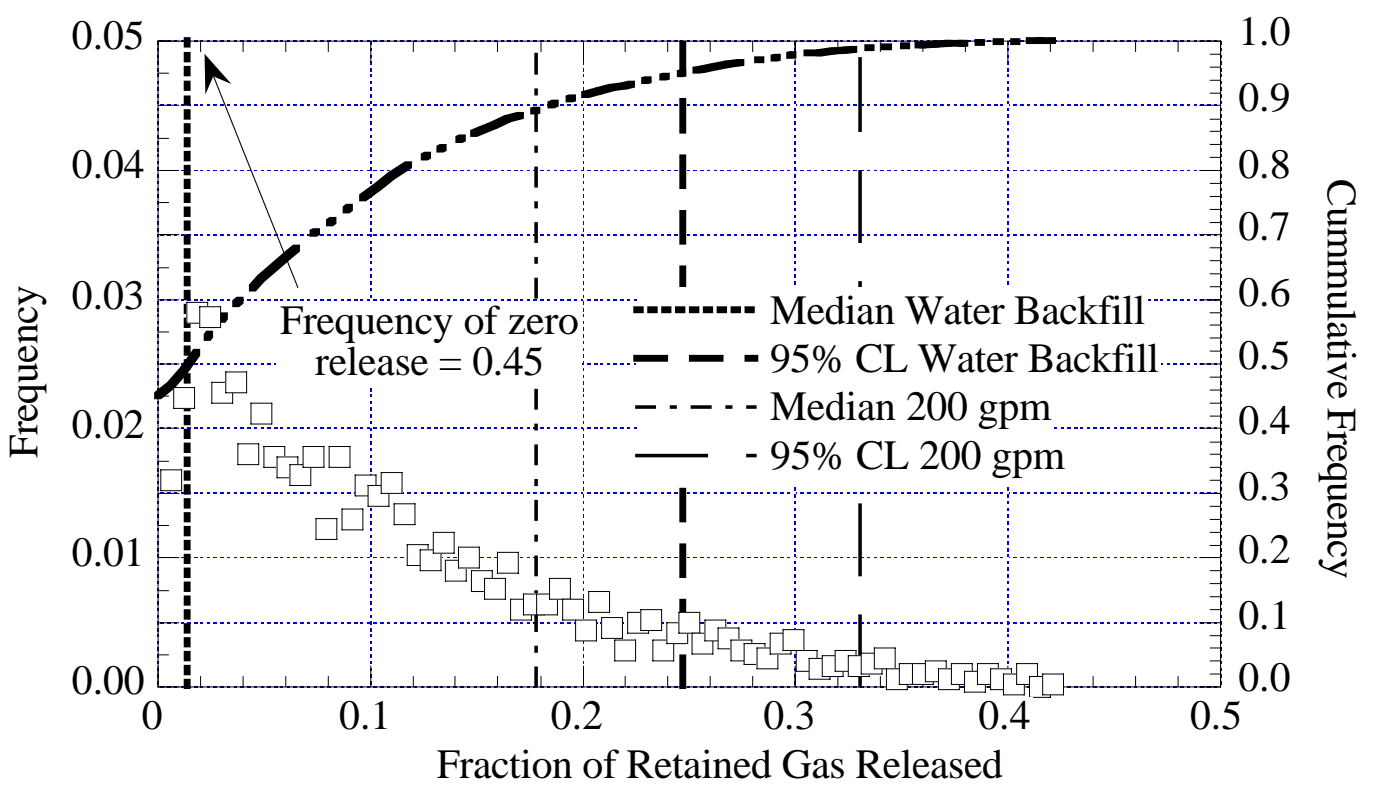

Figure 3.31. Gas Release Fraction for Water Backfill in AN-103 


\subsubsection{AN-104 Decant Results}

The retained gas volume in AN-104 is somewhat larger than in AN-105, but its headspace is larger. The spontaneous gas release history is very similar to that of AN-105. The net result is that AN-104 and AN-105 exhibit similar behavior during decant. The distributions of the peak hydrogen concentration and gas release fraction for the base case are given in Figures 3.32 and 3.33 , respectively. The median and $95^{\text {th }}$ percentile values of the peak hydrogen concentration distribution are 2,800 ppm and 7,100 ppm, respectively. The gas release fraction has a median of 0.12 and a $95^{\text {th }}$ percentile value of 0.27 . A total of about $10 \%$ of the base case runs did not experience a BDGRE during the decant.

As with AN-105, the results for the $30 \mathrm{gpm}$ decant are similar to those of the base case (Figures 3.34 and 3.35. The stop-start control results are shown in Figures 3.36 and 3.37. Approximately $8 \%$ of the runs had hydrogen concentrations that exceeded 6,250. The maximum hydrogen concentration during these stopped periods typically occurred two hours after the decant stopped. The median peak concentration during the stop-start periods was about $7,200 \mathrm{ppm}$, and the 95 th percentile value was just over $25 \%$ of the LFL at 10,400 ppm.

Results for the water backfill control strategy are shown in Figures 3.38 and 3.39. The control strategies do not significantly alter the $95^{\text {th }}$ percentile results, and the median results for the water backfill case return to the background concentration and zero BDGRE gas release, with about $70 \%$ of the simulations having no BDGREs.

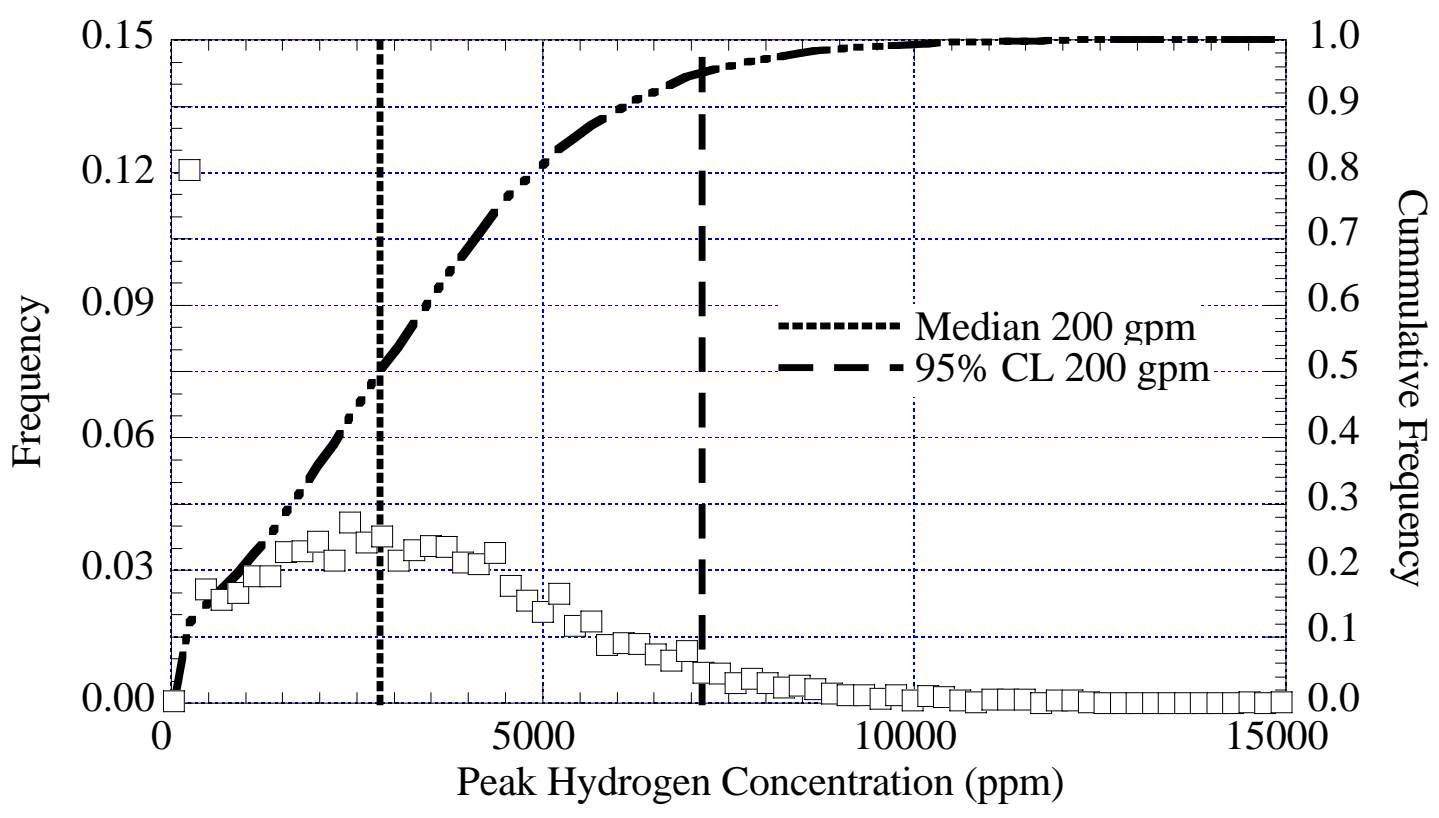

Figure 3.32. Peak Hydrogen Concentration for the AN-104 Base Case 


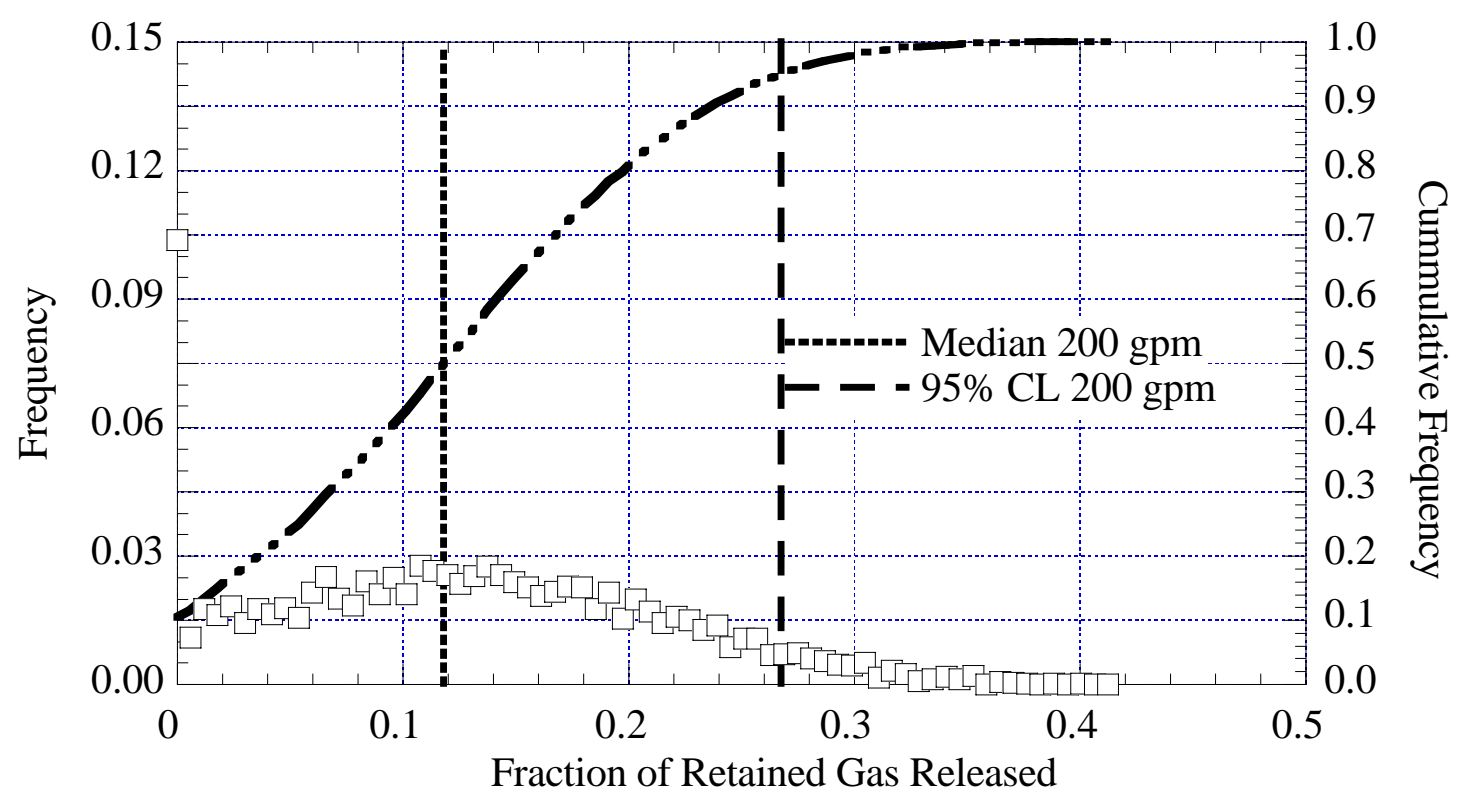

Figure 3.33. Gas Release Fraction for the AN-104 Base Case

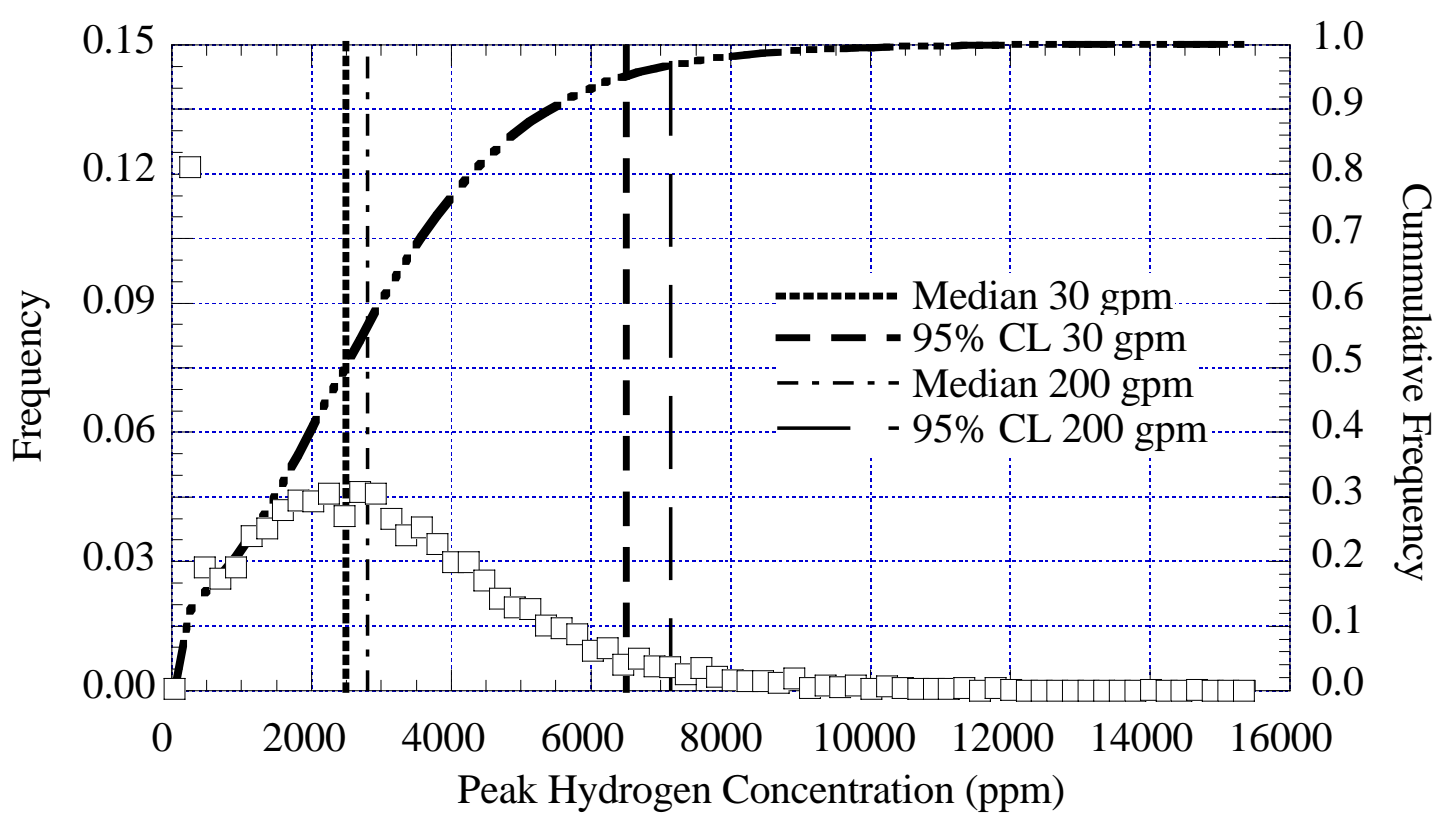

Figure 3.34. Peak Hydrogen Concentration for 30 gpm Decant Rate in AN-104 


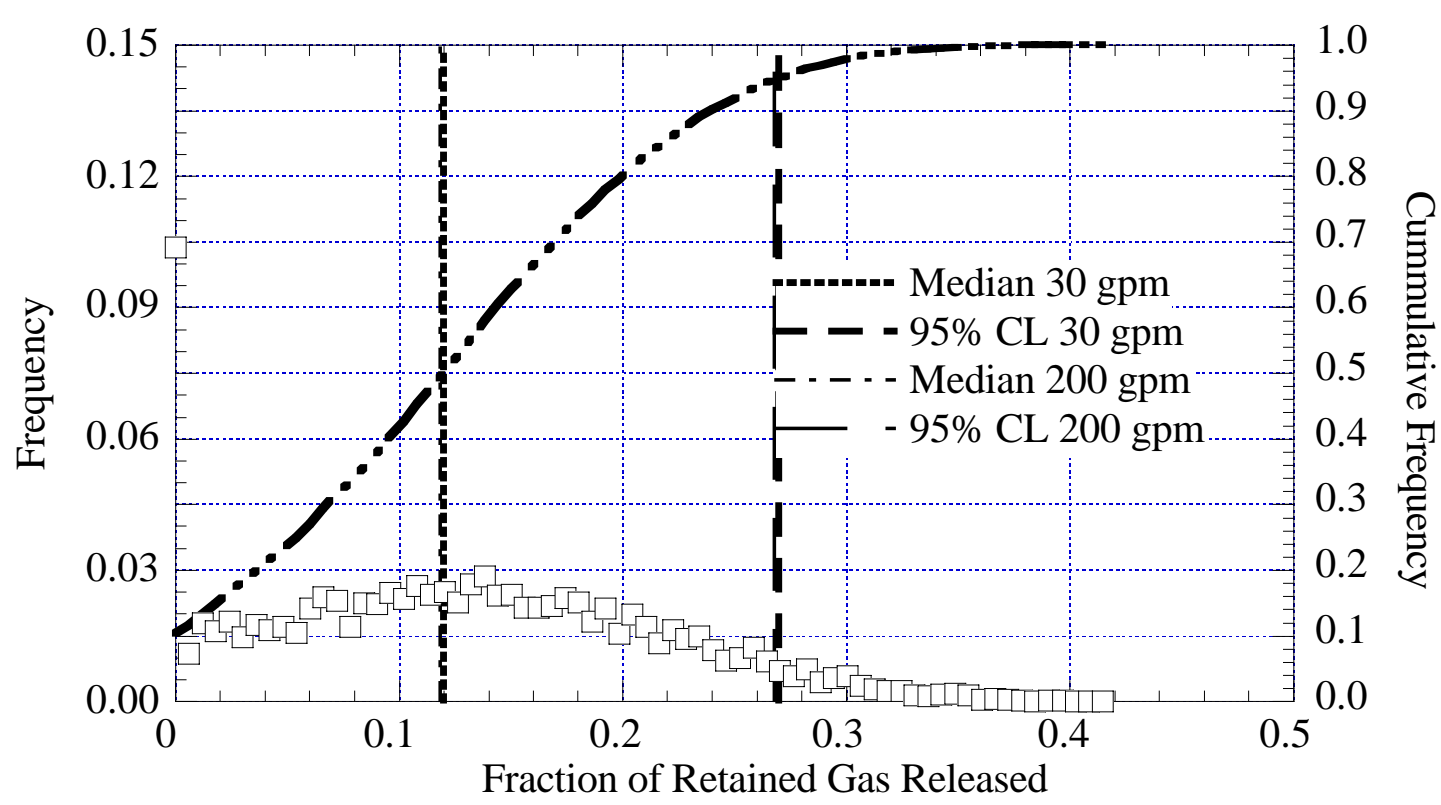

Figure 3.35. Gas Release Fraction for 30 gpm Decant Rate in AN-104

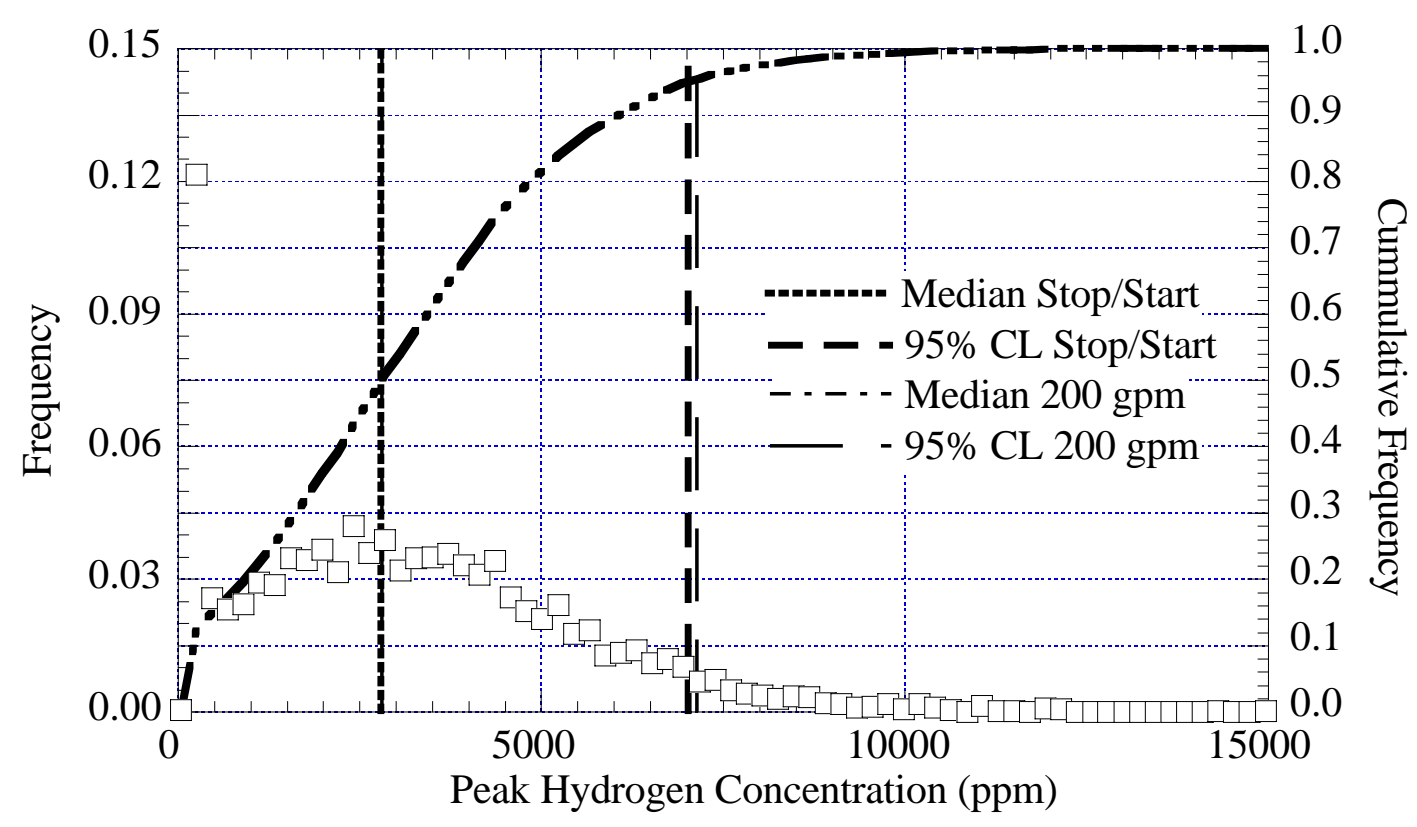

Figure 3.36. Peak Hydrogen Concentration for the Stop-Start Case in AN-104 


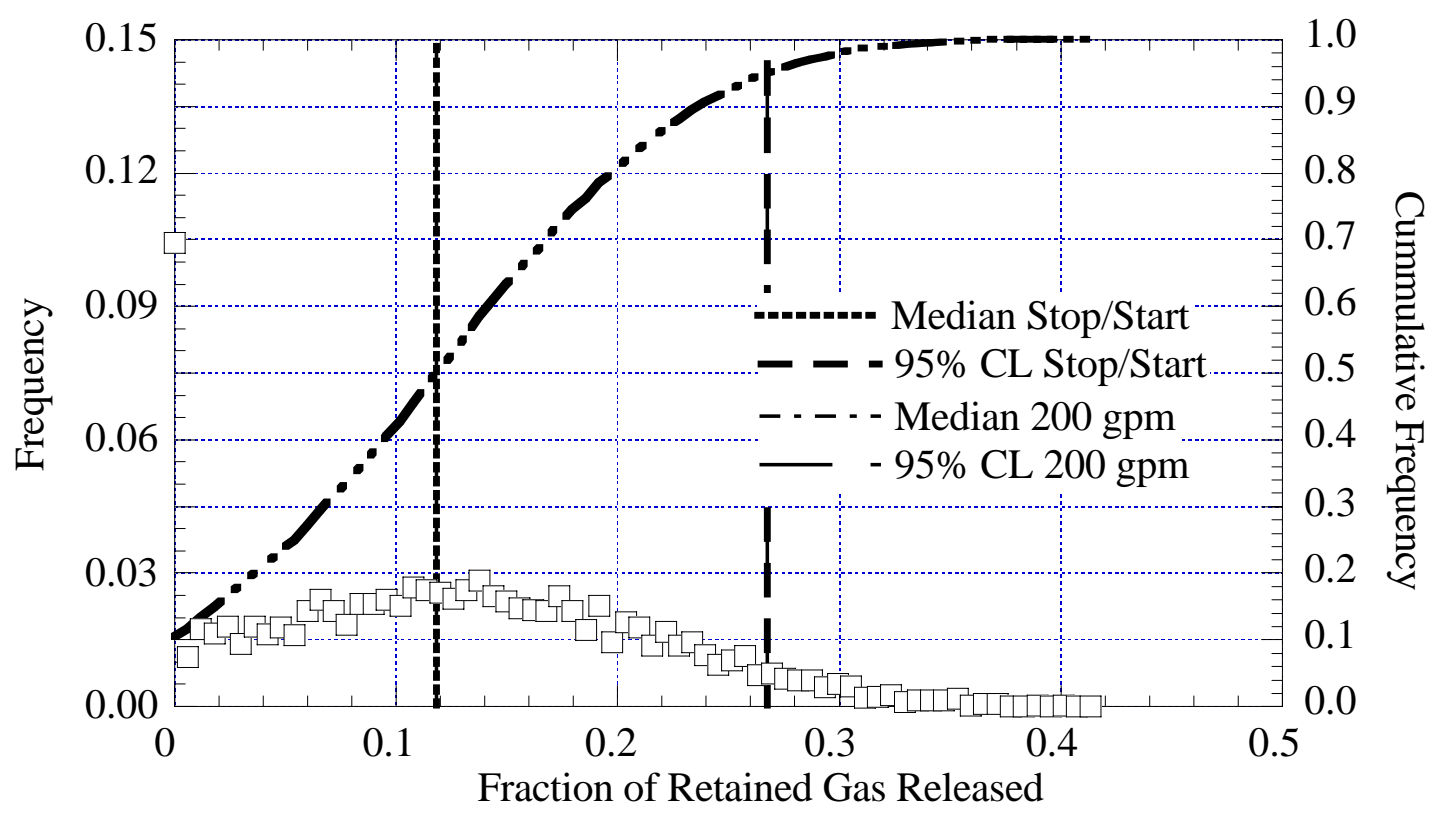

Figure 3.37. Gas Release Fraction for the Stop-Start Case in AN-104

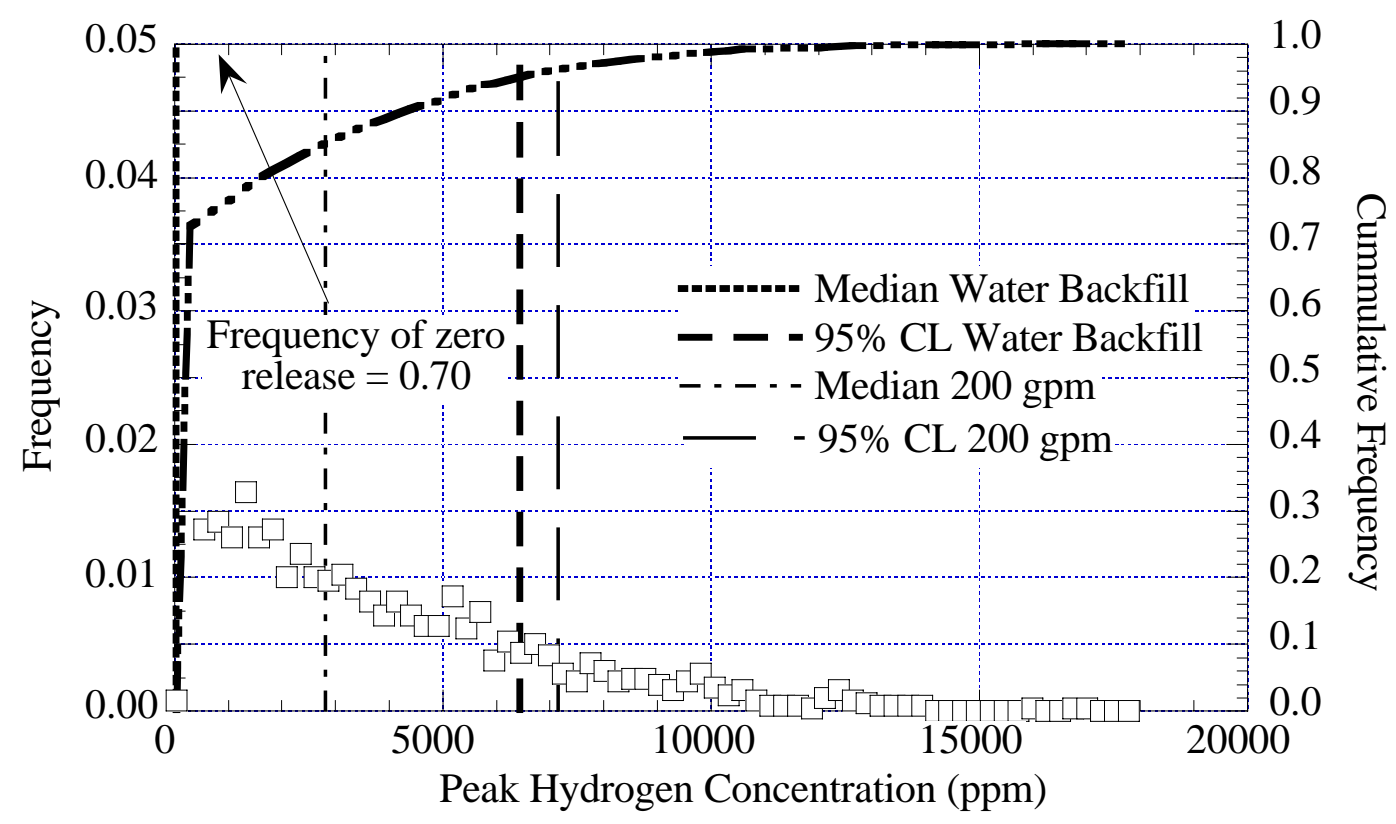

Figure 3.38. Peak Hydrogen Concentration for Water Backfill in AN-104 


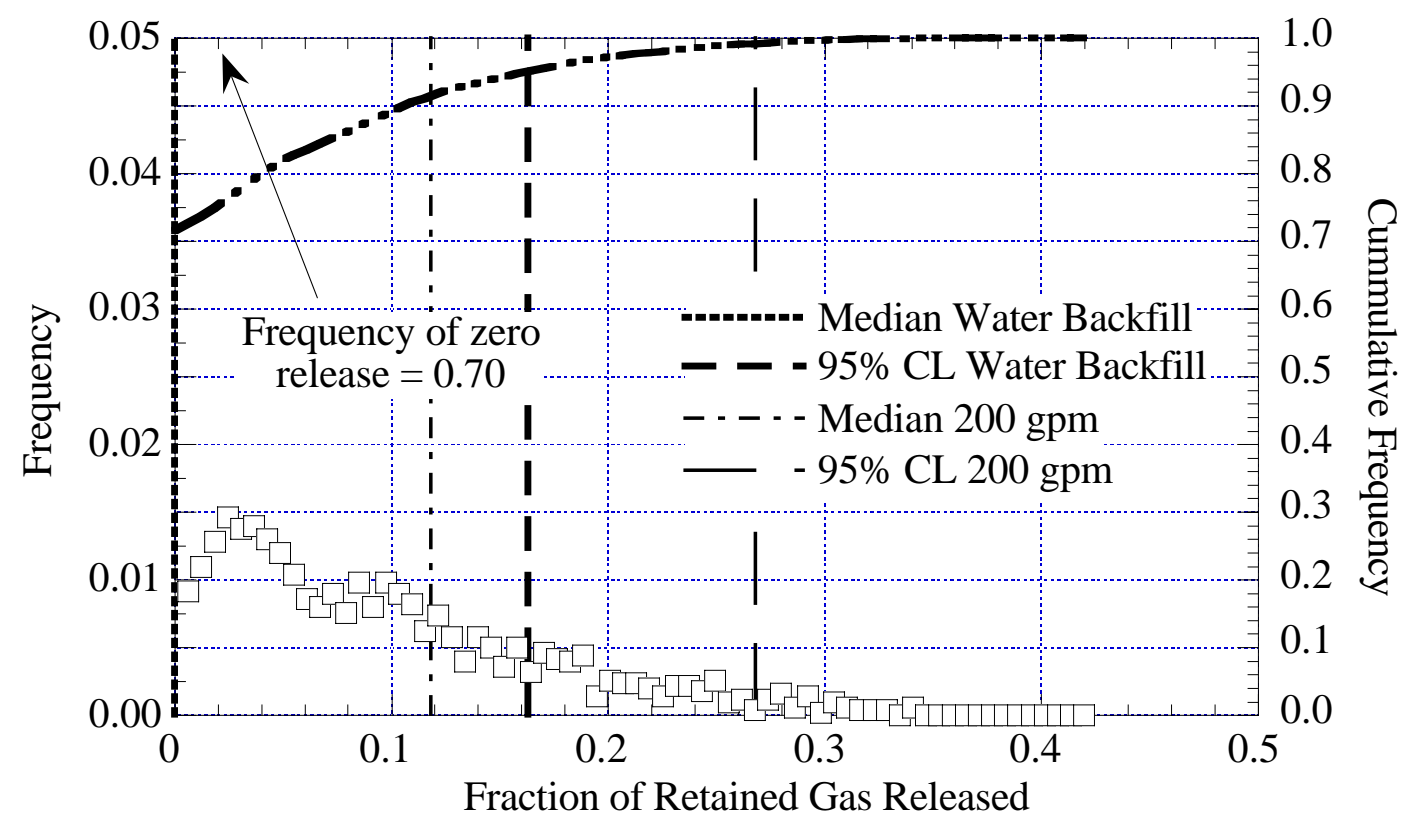

Figure 3.39. Gas Release Fraction for Water Backfill in AN-104

\subsubsection{AW-101 Decant Results}

Tank AW-101 is notable for having the least retained gas volume of the four tanks under consideration. In fact, the stored gas volume is barely sufficient to bring the tank headspace to the LFL if all of it were instantaneously released. Because a much lower fraction of it is released during decant, and the releases are spaced out over a relatively long time, the peak hydrogen concentration is much lower. The distributions of peak hydrogen concentration and gas release fraction for the base case are provided in Figures 3.40 and 3.41, respectively. The median and $95^{\text {th }}$ percentile values of the peak hydrogen concentration are only $400 \mathrm{ppm}$ and $1,500 \mathrm{ppm}$, respectively. Approximately $15 \%$ of the runs did not have a BDGRE. The median gas release fraction was 0.05 and 0.17 at the $95^{\text {th }}$ percentile.

As shown in Figures 3.42 and 3.43, the results for the 30 gpm decant are similar to those of the base case. The stop-start control was not applicable to this tank, as the maximum concentration did not exceed $6,250 \mathrm{ppm}$ in the base case. The water backfill control results are shown in Figures 3.44 and 3.45. Both the $95^{\text {th }}$ percentile and median water backfill results are significantly lower than the base case as $86 \%$ of the runs had no BDGREs occur, and gas release was significantly reduced in those runs that did have BDGREs. 


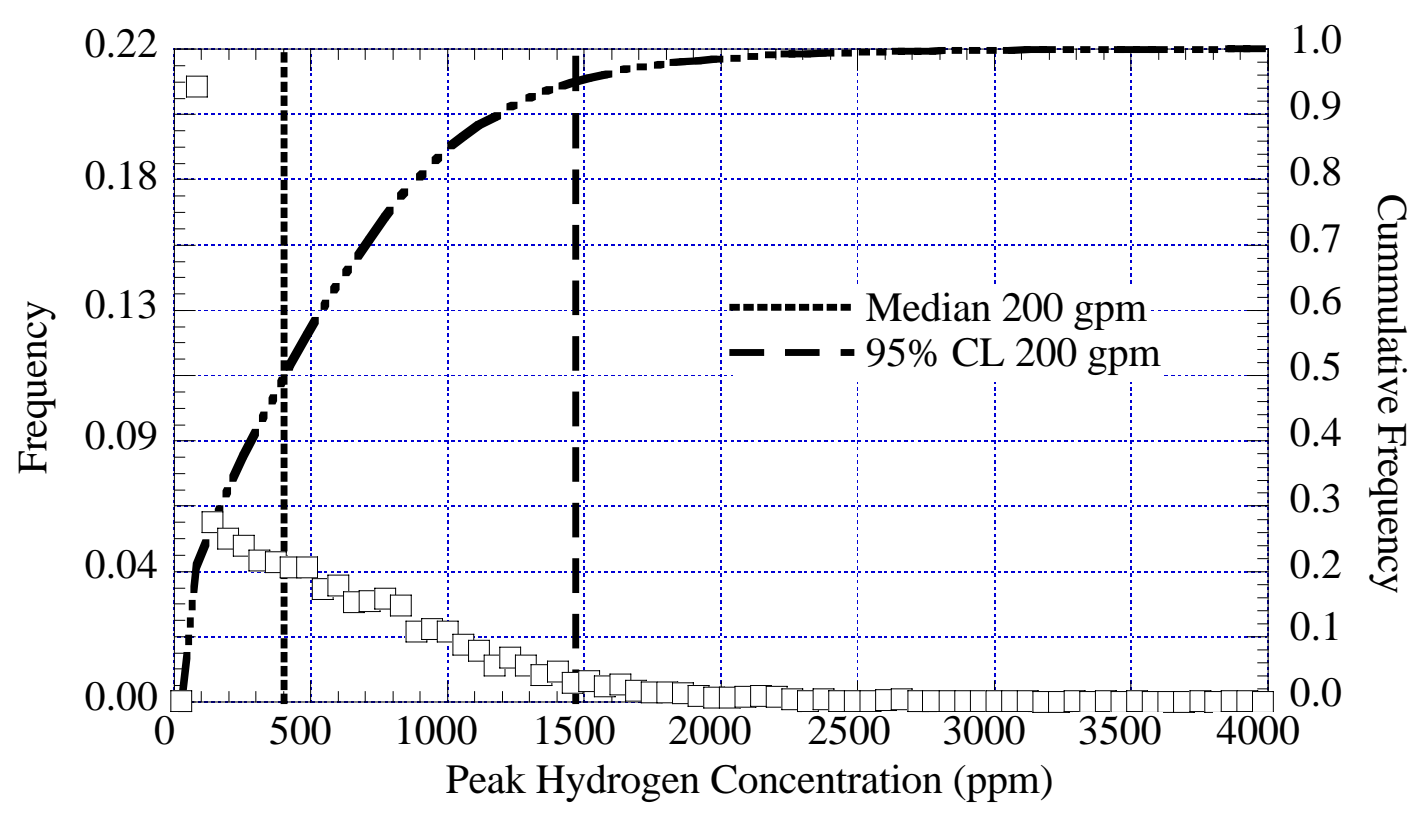

Figure 3.40. Peak Hydrogen Concentration for the AW-101 Base Case

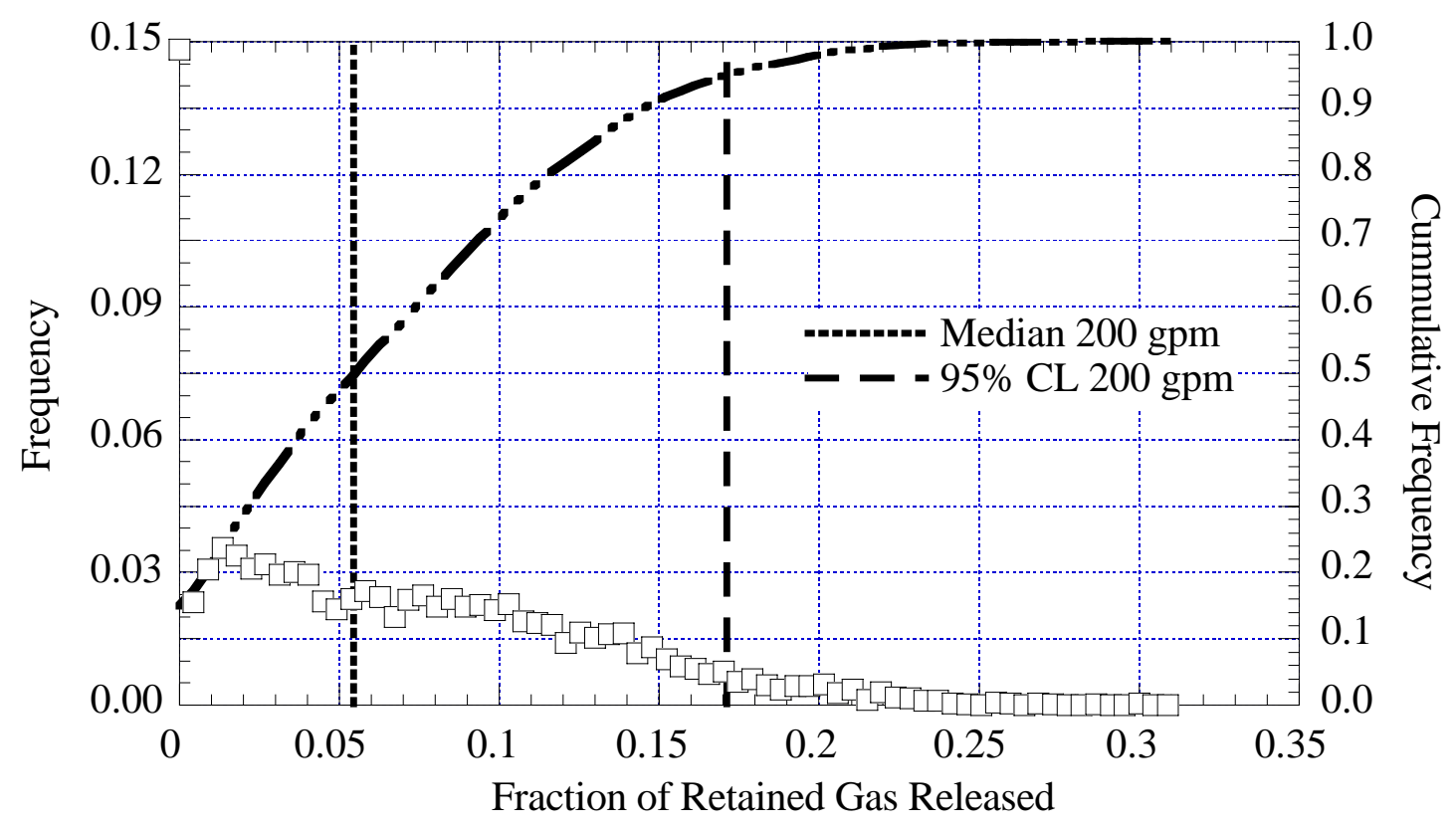

Figure 3.41. Gas Release Fraction for the AW-101 Base Case 


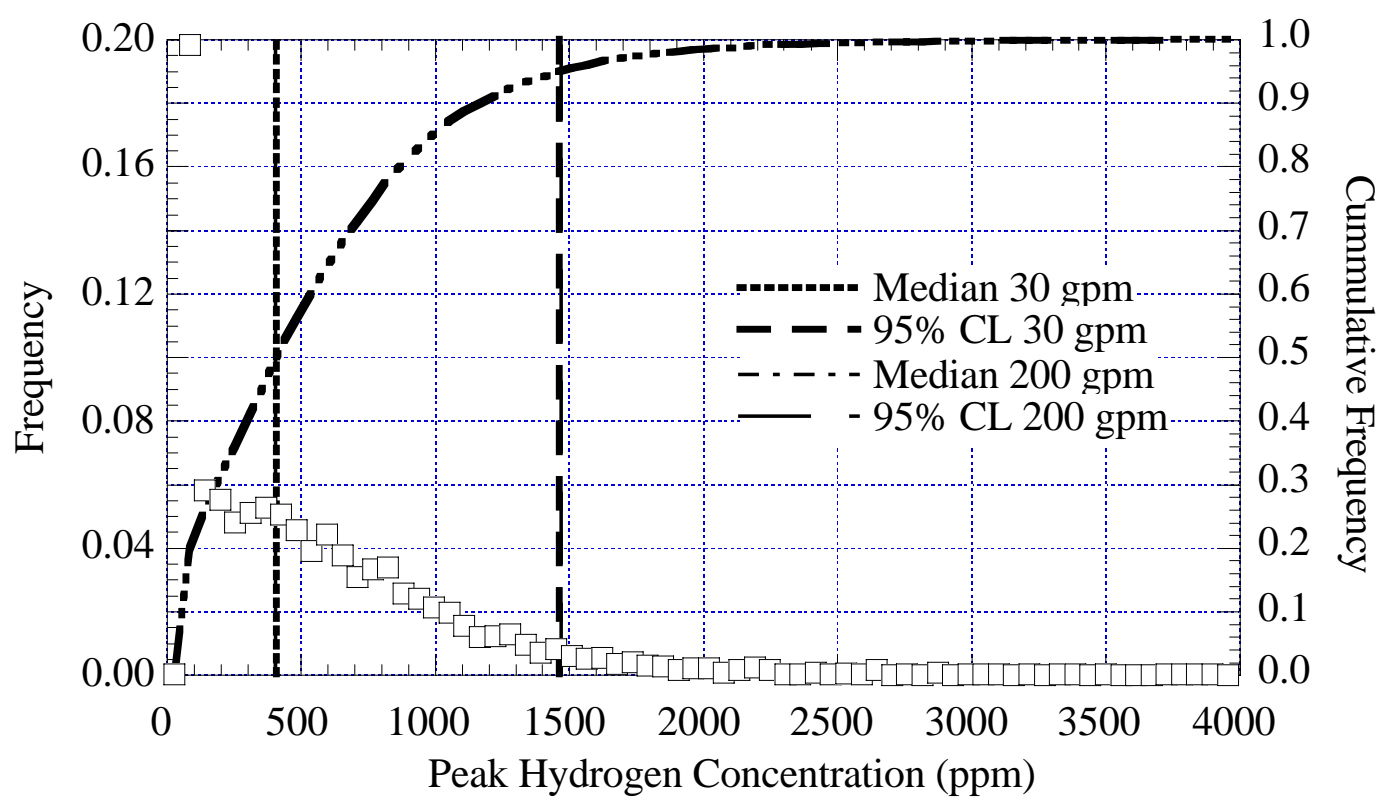

Figure 3.42. Peak Hydrogen Concentration for 30 gpm Decant Rate in AW-101

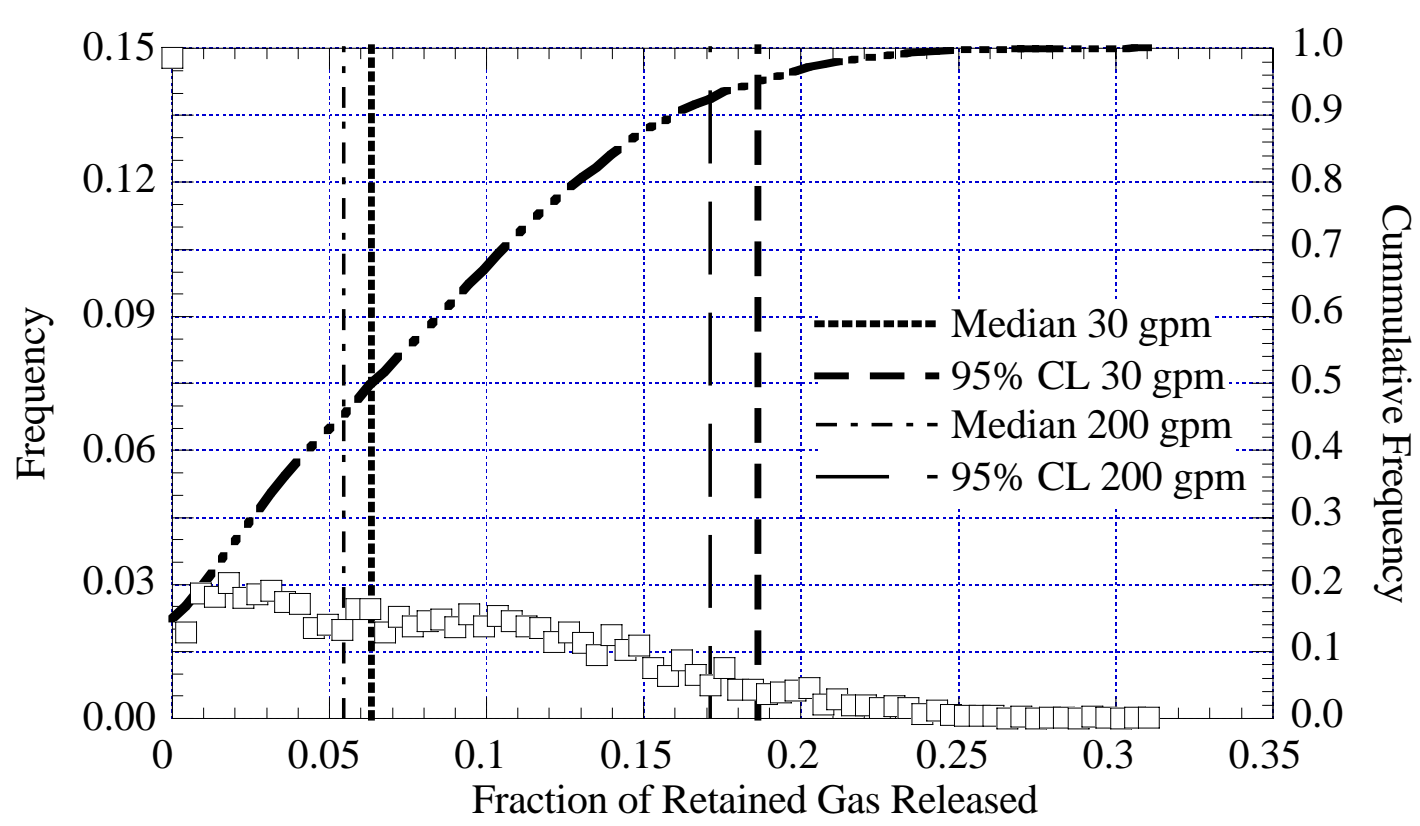

Figure 3.43. Gas Release Fraction for 30 gpm Decant Rate in AW-101 


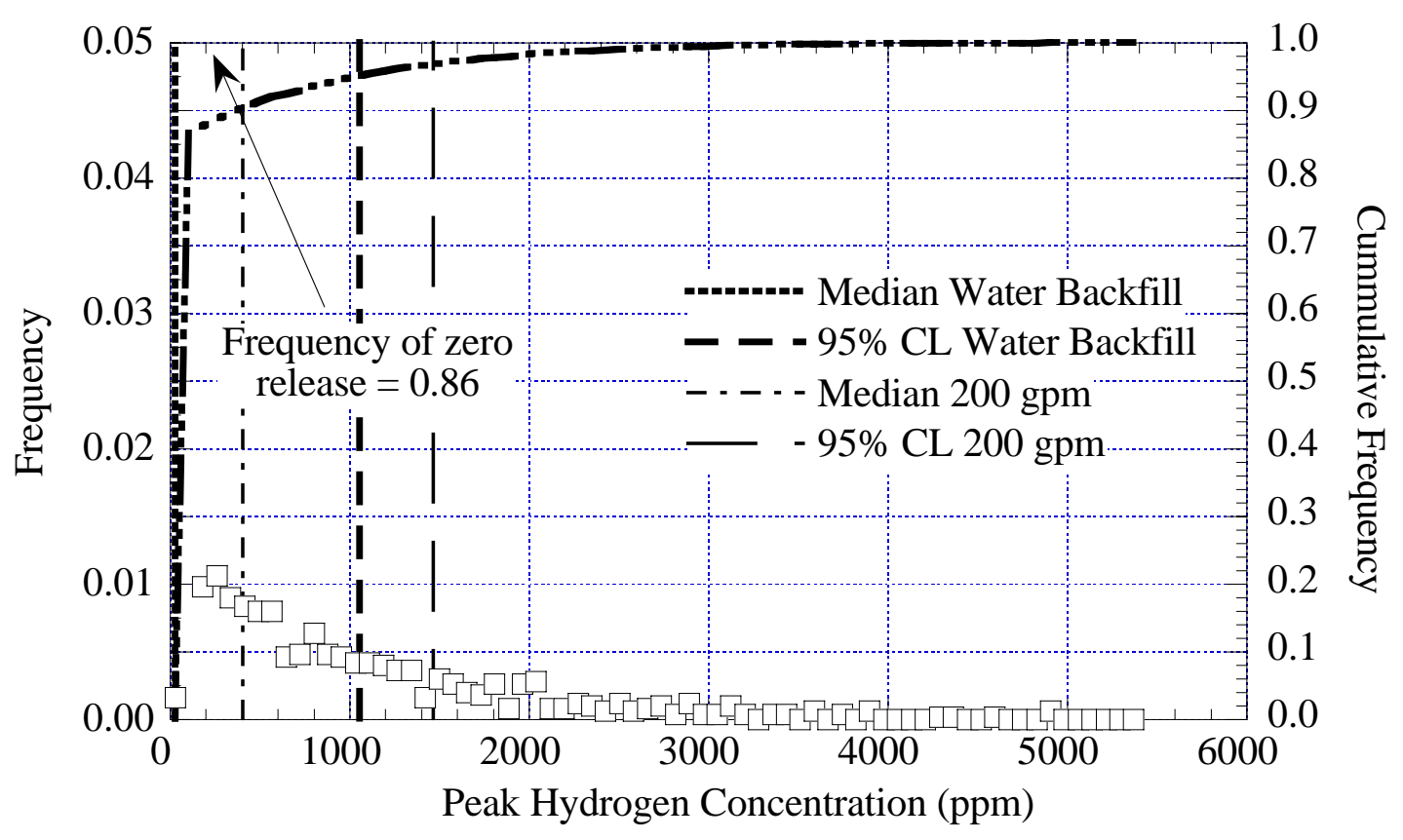

Figure 3.44. Peak Hydrogen Concentration for Water Backfill in AW-101

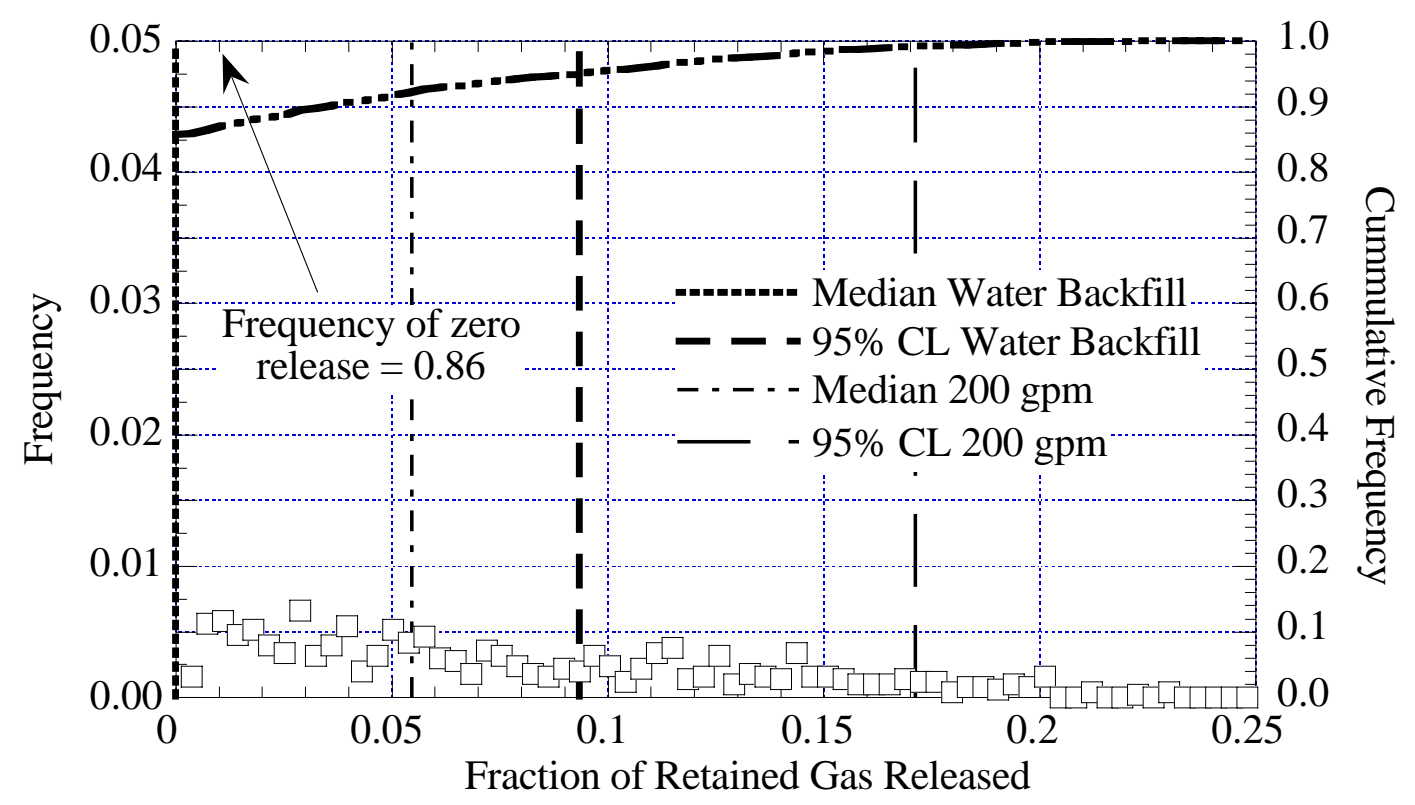

Figure 3.45. Gas Release Fraction for Water Backfill in AW-101 


\subsection{Conclusions from Supernate Decant Modeling Results}

The numerical results are summarized in Table 3.9. Except for AN-103, the peak hydrogen concentrations resulting from gas releases predicted to be induced by supernate decant correspond roughly to those produced by the larger spontaneous GREs. AN-103 is an exception because its history of very small, infrequent gas releases does not match the expectation based on its waste configuration. Nevertheless, none of the runs for AN-103 reached the LFL, even though the majority of the runs had BDGREs occur relatively early in the decant. AW-101 lies

Table 3.9. Summary of Decant Analysis Results

\begin{tabular}{|c|c|c|c|c|}
\hline Tank & Run & Quantity & Median & $95 \% \mathrm{CL}$ \\
\hline \multirow{8}{*}{$\overline{\mathrm{AN}-105}$} & Base Case & Hydrogen (ppm) & 2,800 & 8,300 \\
\hline & & Fraction Gas Release & 0.09 & 0.22 \\
\hline & 30 gpm Decant & Hydrogen (ppm) & 2,600 & 8,000 \\
\hline & & Fraction Gas Release & 0.09 & 0.22 \\
\hline & Stop-Start Control & Hydrogen (ppm) & 2,800 & 8,200 \\
\hline & & Fraction Gas Release & 0.09 & 0.22 \\
\hline & Water Backfill & Hydrogen (ppm) & 23 & 7,600 \\
\hline & & Fraction Gas Release & 0.00 & 0.13 \\
\hline \multirow[t]{8}{*}{ AN-104 } & Base Case & Hydrogen (ppm) & 2,800 & 7,100 \\
\hline & & Fraction Gas Release & 0.12 & 0.27 \\
\hline & 30 gpm Decant & Hydrogen (ppm) & 2,500 & 6,500 \\
\hline & & Fraction Gas Release & 0.12 & 0.27 \\
\hline & Stop-Start Control & Hydrogen (ppm) & 2,800 & 7,000 \\
\hline & & Fraction Gas Release & 0.12 & 0.27 \\
\hline & Water Backfill & Hydrogen (ppm) & 34 & 6,400 \\
\hline & & Fraction Gas Release & 0.00 & 0.16 \\
\hline \multirow[t]{8}{*}{ AN-103 } & Base Case & Hydrogen (ppm) & 10,500 & 21,100 \\
\hline & & Fraction Gas Release & 0.18 & 0.33 \\
\hline & $30 \mathrm{gpm}$ Decant & Hydrogen (ppm) & 8,200 & 19,100 \\
\hline & & Fraction Gas Release & 0.18 & 0.33 \\
\hline & Stop-Start Control & Hydrogen (ppm) & 9,000 & 19,400 \\
\hline & & Fraction Gas Release & 0.18 & 0.33 \\
\hline & Water Backfill & Hydrogen $(\mathrm{ppm})$ & 1,500 & 21,600 \\
\hline & & Fraction Gas Release & 0.01 & 0.25 \\
\hline \multirow[t]{8}{*}{ AW-101 } & Base Case & Hydrogen (ppm) & 400 & 1,500 \\
\hline & & Fraction Gas Release & 0.05 & 0.17 \\
\hline & $30 \mathrm{gpm}$ Decant & Hydrogen (ppm) & 400 & 1,500 \\
\hline & & Fraction Gas Release & 0.06 & 0.19 \\
\hline & Stop-Start Control & Hydrogen (ppm) & Not Applicable & Not Applicable \\
\hline & & Fraction Gas Release & Not Applicable & Not Applicable \\
\hline & Water Backfill & Hydrogen (ppm) & 27 & 1,100 \\
\hline & & Fraction Gas Release & 0.00 & 0.09 \\
\hline
\end{tabular}


at the other extreme in that its predicted peak hydrogen concentrations are far below even the 6,250-ppm action level. These analysis results indicate that removing the supernatant liquid from these four tanks will not induce gas releases that cause the tank headspace to reach the LFL.

These results are not sensitive to the decant rate, and the peak hydrogen concentration is not reduced by stopping the decant at the action level of 6,250 ppm hydrogen. However, backfilling with water during the decant has a significant probability of preventing BDGREs, though the peak hydrogen concentration approximates the base case if they do occur (excepting AW-101). 


\subsection{Analysis of Gas Releases Induced by Mixer Pump Operation}

Jet mixing is a mechanical disturbance that mobilizes sediment by creating hydrodynamic shear and pressure forces that exceed the strength of the material. This disruption of sediment releases retained gas bubbles that eventually enter the tank headspace. Thus gas release is expected from the volume of waste actually disrupted. A powerful mixer pump may potentially affect a large waste volume at a relatively high rate, which, by design, suspends a large fraction of the mobilized solids in the liquid layer. As described in Section 2.1, this increases the density of the supernate, reducing the neutral buoyancy gas fraction and potentially inducing BDGREs in gobs that were not buoyant when mixing started.

The model developed to represent this process treats gas releases from both the direct disturbance of the waste by the mixer pump and from subsequent induced BDGREs. It consists of two independent parts: the first estimates the volume of waste actually mobilized by a mixer pump of known characteristics running at a given speed and duration; the second determines the headspace hydrogen concentration resulting from a specified schedule of pump runs, each of which mobilizes a given volume of waste. The first model is built on jet theory and is described in Section 4.1. The second is a direct adaptation of the detailed supernate decant model derived in Section 3.1, with relatively minor modifications as described in Section 4.2. Parameters for the gas release model are defined in Section 4.3. Results of applying the model to mixing in AN-105 are presented in Section 4.4 and those for the other three tanks in Section 4.5. Conclusions on mixer pump induced gas releases are given in Section 4.6.

\subsection{Modeling the Effect of the Mixer Pump Jet on the Waste}

Ideally, when the mixer pump is initially operated, one would like to know how much gas will be released in terms of the mixer pump operational parameters such as flow rate (or motor $\mathrm{rpm}$ ), run time, and nozzle orientation. In that way, the pump can be operated in a safe manner. From a fluid mechanics point of view, one would like to understand the role of the waste configuration and physical properties as well. These include waste strength, layer densities, rheology, etc.

In this section we attempt to develop a working model for predicting gas release as a function of all the pertinent operational parameters, waste configuration, and waste physical properties. This is a complex undertaking because the physical system is complex and limited data are available for validation. However, by applying fairly straightforward physical principles, a useful model can be developed for gas release during mixer pump operation. We attempt to validate the model against a very limited data set from initial mixer pump operation in Tank SY-101 and present recommendations for further model validation. 


\subsubsection{The Theory of Jet-Induced Gas Release}

In this section we review and extend the basic theory of jet-induced gas release. The goal is to provide the theoretical basis to develop a working model for predicting gas release during mixer pump operation.

\subsubsection{Important Physical Parameters}

In general, we would expect gas release during mixer pump operation to depend on a number of physical parameters. These parameters and an explanation of their importance are as follows:

Discharge velocity $\left(\mathbf{U}_{0}\right)$ : The velocity of fluid discharging from the mixer pump nozzles is important for several reasons. Energy and momentum imparted to the gas-retaining sediment layer depend upon the local fluid velocities. Local velocities, in turn, scale with discharge velocity. Discharge velocity also determines the volume or mass flow rate. These can be important because the physical volume of the discharge must displace some waste, potentially releasing gas in the process. For a given pump operating in a given fluid, discharge velocity will be proportional to motor rpm; hence they can be used somewhat interchangeably.

Nozzle diameter $\left(\mathbf{d}_{0}\right)$ : The nozzle diameter is important because the total hydraulic power depends on the nozzle area. The nozzle diameter also determines the scale for velocity decay. Additionally, mass and volume flow rate depend on nozzle diameter.

Density $\left(\rho_{0}\right)$ : The density of the jet discharge contributes to the momentum, energy, hydraulic power, and mass flow rate. The density of the waste affects its inertia. The relative density of the discharging fluid $(\Delta \rho)$ and the surrounding waste dictates buoyancy, which can have a significant effect on jet behavior.

Yield strength $(\boldsymbol{\tau})$ : The yield strength of the sediment layer is important for several reasons. First, if the yield strength is large, it is more difficult for a jet to mobilize and break up the sediment layer material; hence it releases less gas. Also, the propensity for the sediment layer material to cave in after being eroded by the jet increases with decreasing yield strength. Finally, the strength of the solid/liquid matrix determines how firmly gas bubbles are held in place.

Viscosity $(\mu)$ : The viscosity of the waste is unimportant as long as fluid velocities are high. Here the hydrodynamic forces are dominated by inertia resulting in turbulent conditions. As jet velocities decrease (far from the nozzle) viscous forces come into play. The magnitude of the viscosity (or effective viscosity for materials with complex rheology) affects mixing and velocity decay far from the nozzle.

Gas (void) fraction $(\alpha)$ : Gas release during mixer pump operation is clearly affected by the gas fraction in the sediment layer. All things being equal, we would expect waste with larger void fraction to release more gas during a mixer pump operation than waste with a smaller void fraction. 
Pressure (p): The average pressure at which gas is stored in the sediment layer is important because it contributes to the total number of moles of gas at a given void fraction. During gas release, the volume of released gas expands into the tank headspace, with final volume depending on initial pressure.

Pump run time (t): Pump run time is important to gas release during mixer pump operation. The longer the pump is operated, the more mass, fluid volume, and hydraulic power are delivered to the waste. The rate of gas release likely changes during a pump run. Eventually, gas release likely diminishes for long pump run times.

In general then, we can say that the total gas release volume during mixer pump operation should depend on the above-mentioned parameters. This is expressed mathematically as

$$
\mathrm{V}_{\text {rel }}=\mathrm{f}\left(\mathrm{U}_{0}, \mathrm{~d}_{0}, \rho_{0}, \Delta \rho, \tau, \mu, \alpha, \mathrm{p}, \mathrm{t}\right)
$$

\subsubsection{Important Physical Processes}

The function in Eq. (4.1) can be found if we model the conservation laws adequately for mass, momentum, and energy. In fluid dynamics, these laws form the Navier-Stokes equations. In the case of a multiphase medium (gas, liquid, and pseudo solid), the equations become exceedingly complex and certainly intractable. As an alternative, we consider simplified expressions for the important physical mechanisms and processes.

Mass flow: Mass is conserved during mixer pump operation. The total flow rate of liquid, solids, and gas discharging from the nozzle is equal to the total flow rate of material entering the pump inlet. If the density of the discharge and inlet streams is the same, the volume flow rate is also the same. The mass flow rate is given by

$$
\dot{\mathrm{m}}_{0}=\rho_{0} \mathrm{U}_{0} \mathrm{~A}_{0}
$$

where $\mathrm{A}_{0}=\frac{\pi}{4} \mathrm{~d}_{0}^{2}$ is the nozzle area. The total mass of fluid that is discharged in time $\mathrm{t}$ is

$$
M(t)=\rho_{0} U_{0} A_{0} t
$$

Similarly, the volume flow rate is given by

$$
\mathrm{Q}_{0}=\mathrm{U}_{0} \mathrm{~A}_{0}
$$

and the total volume of fluid discharged during time $t$ is

$$
\mathrm{V}(\mathrm{t})=\mathrm{U}_{0} \mathrm{~A}_{0} \mathrm{t}
$$

Because volume is approximately conserved, Eq. (4.5) sets an upper bound on the volume of waste that can be disturbed during mixer pump operation. 
Momentum flux: Moving fluids are associated with momentum. This momentum creates forces on the sediment layer material as the fluid impinges on it. The momentum flux discharging from the nozzle is given by

$$
\mathrm{P}=\rho_{0} \mathrm{U}_{0}^{2}
$$

The momentum flux has units of pressure and in fact can be thought of as a pressure. The momentum flux is independent of nozzle area.

Kinetic energy: The kinetic energy flux of the discharging fluid from the nozzle is given by

$$
\mathrm{ke}=\frac{1}{2} \rho_{0} \mathrm{U}_{0}^{3}
$$

The power of the discharge is given by

$$
\dot{\mathrm{P}}_{\mathrm{k}}=\frac{1}{2} \rho_{0} \mathrm{U}_{0}^{3} \mathrm{~A}_{0}
$$

The power shown in Eq. (4.8) is approximately equal to the power produced by the pump minus any losses in the ductwork and an amount that accounts for pressure differences between the inlet and nozzle.

The total kinetic energy discharged in time $t$ is

$$
\mathrm{KE}(\mathrm{t})=\frac{1}{2} \rho_{0} \mathrm{U}_{0}^{3} \mathrm{~A}_{0} \mathrm{t}
$$

The energy shown in Eq. (4.9) is not conserved downstream of the jet discharge. As soon as the fluid discharges the nozzle, turbulent motions, slip between solid and liquid phases, viscous shear stresses, and other irreversible interactions cause the energy to be dissipated. Even so, Eq. (4.9) sets the upper limit on the amount of energy delivered to the sediment layer material matrix.

Potential Energy: If the density of the discharging fluid is different from the density of the surrounding material in the sediment layer, the jet will posses potential energy. This potential energy can be either negative (dense jet) or positive (buoyant jet). The potential energy flux is given by

$$
\text { pe }=\left(\rho_{0}-\rho_{S}\right) g h U_{0}
$$

where $\rho_{\mathrm{S}}$ is the density of the sediment layer, $g$ is the gravitational constant, and $\mathrm{h}$ can be thought of as the distance from the nozzle to the top of the sediment layer. The rate of buoyant energy production, or "buoyant power," is given by

$$
\dot{\mathrm{P}}_{\mathrm{b}}=\left(\rho_{0}-\rho_{\mathrm{S}}\right) \mathrm{ghU}_{0}^{2} \mathrm{~A}_{0}
$$


and the total buoyant energy discharged from the nozzle is

$$
\operatorname{PE}(t)=\left(\rho_{0}-\rho_{S}\right) g h U_{0}^{2} A_{0} t
$$

To get an idea of the relative magnitudes of the kinetic and potential energy, we divide Eq. (4.9) by Eq. (4.12) to obtain

$$
\mathrm{PE} / \mathrm{KE}=\frac{\left(\rho_{0}-\rho_{\mathrm{S}}\right)}{\rho_{0}} \frac{2 \mathrm{gh}}{\mathrm{U}_{0}}
$$

For typical conditions for retrieval systems in DSTs $\left(\mathrm{U}_{0} \sim 10-20 \mathrm{~m} / \mathrm{s}, \mathrm{h} \sim 2-4 \mathrm{~m}, \rho_{0} \sim 1.4-1.6\right.$ $\mathrm{g} / \mathrm{cc}, \rho_{\mathrm{s}} \sim 1.6-1.7 \mathrm{~g} / \mathrm{cc}$ ), the ratio ranges from about $0-1.7$. Hence potential energy can be a significant factor, depending primarily on the relative density of the discharge and surrounding material.

Strain energy: For gas to be released from the sediment layer material, energy, or work, is required to deform the material. The minimum energy required to deform gas-retaining sediment layer material of volume $\mathrm{V}$ is approximately $\tau \mathrm{V}$. The rate of work, or power for yielding the material, is given by

$$
\dot{\mathrm{P}}_{\mathrm{y}}=\tau \mathrm{u}_{\mathrm{f}} \mathrm{S}
$$

where $\mathrm{u}_{\mathrm{f}}$ is the speed of the yielding front and $\mathrm{S}$ is the surface area. The work done on the sediment layer material as a function of time is given by

$$
\mathrm{YE}(\mathrm{t})=\tau \mathrm{u}_{\mathrm{f}} \mathrm{St}
$$

Equations (4.14) and (4.15) set a lower bound on the amount of energy or power required to release the gas stored in a given volume of sediment layer material. This energy, at a minimum, must be supplied by the mixer pump. Of course, because energy is not conserved, the actual amount of energy required will be greater than the strain energy.

Onset of Yield: For material to begin to yield, the pressure acting on the material surface must exceed the yield strength. The pressure of a jet is made up of both static and dynamic components. The static component is negligible because both the moving fluid and the static, unyielded sediment layer material will be at the same pressure. The dynamic component of pressure is $1 / 2 \rho u^{2}$, where $\rho$ is the local jet density and $u$ is the local jet velocity. The dynamic pressure must exceed the yield strength for deformation to occur. This leads to the following requirement for local sediment layer yield:

$$
\rho u^{2} / 2 \tau>1
$$

Equation (4.16) relates to the normal stress acting on a surface of sediment layer material. Shear stresses resulting from jet flow also may erode the sediment layer and will generally be 
proportional to $1 / 2 \mathrm{\rho u}^{2}$, with the constant of proportionality being the friction coefficient. Hence, a condition similar to Eq. (4.16) can be written if the friction coefficient is known.

Entrainment: Another potential mode of sediment layer yield leading to gas release is the phenomenon of entrainment. Entrainment is similar to erosion by shear stress but is due to a fundamentally different mechanism. Entrainment in turbulent jets results from large-scale turbulent eddies that engulf regions of quiescent fluid that are outside the core of the jet. It is not known what role entrainment plays when a turbulent jet is surrounded by a pseudo solid. One likely criterion is that the local pressure fluctuation due to eddies is greater than the yield stress. Because pressure fluctuations scale locally with $1 / 2 \rho \mathrm{u}^{2}$ for Newtonian turbulent flows, it is reasonable to assume a condition similar to Eq. (4.16) applies to entrainment as well.

Collapse: Another likely mechanism for sediment layer yield is collapse. As the jet bores into the sediment layer by some combination of the processes and mechanisms discussed above, material above should eventually collapse into the jet cavity. The collapsed material may release gas spontaneously during the collapse or during subsequent jet action. Collapse generally occurs when the lithostatic pressure differences exceed the yield stress locally. Because most sediment layers have relatively low yield stress (40 to $300 \mathrm{~Pa}$ ), it is fairly easy for material to collapse.

\subsubsection{System Configuration Issues}

In addition to the physical processes and mechanisms described in the previous section, there are a number of other factors associated with the mixer pump system that affect gas release behavior. The first is the orientation of the discharge. Nozzles aimed toward the center of the tank will likely produce gas release in different amounts than those aimed at or near a wall. The wall necessarily will turn the jet flow upward and along the wall. The elevation of the nozzles above the tank bottom is also important. Jet flows parallel to a solid surface generally turn toward that surface and attach themselves. The characteristics of these attached jet flows are somewhat different from jet flows that are not influenced by solid surfaces.

For several reasons, perhaps the most important system configuration issue is the location of the pump inlet. First, if the inlet is far from the nozzle (either higher or lower), the density of the discharge will likely be different from that of the surrounding waste. If the inlet is near the surface or above the sediment layer, supernatant will be drawn into the pump. This is the case for the mixer pump installed in Tank SY-101. Because the supernate may be significantly less dense than the sediment layer, buoyant effects may be very significant in or even dominate the resulting jet flow structure. Aside from buoyancy, the need to conserve volume may also force the jet up through the sediment layer to provide a return path for the discharged fluid.

If the mixer pump inlet is located near the tank bottom (such as is planned for waste retrieval operations), density effects will be minimized. However, another phenomenon may occur. The possibility of discharge "short circuiting" is increased for this situation. The jet may act more like a confined jet, where at some distance from the nozzle the jet stops, turns, and then returns to the inlet. Under these conditions, the gas release characteristics could vary significantly from a case where the inlet is in the supernatant like it was in SY-101. 


\subsubsection{Observations and Data from Initial Mixing of SY-101}

Because the initial phases of mixer pump testing in SY-101 released gas from the waste, as intended, the tank data taken during that period are potentially useful in understanding and predicting gas releases from other tanks as they are degassed by mixing. Our objective in reviewing the SY-101 data was to glean any correlations between any of the pump operational parameters and the amount of gas released. These data can potentially be used to validate or calibrate a working model of mixer-pump-induced gas release.

Caley et al. (1998) were the first to use the SY-101 pump testing data as a way to estimate possible gas releases during mixer pump degassing of AN-105. They noted that the maximum daily gas release during high-speed mixer pump testing in SY-101 (Phase B) was $14.3 \mathrm{~m}^{3}$ $\left(408 \mathrm{ft}^{3}\right)$ and that it was produced by a run that delivered $18.2 \mathrm{~kW}-\mathrm{hr}(24.4 \mathrm{hp}-\mathrm{hr})$ of energy to the waste. Because this same amount of energy is supplied to the AN-105 waste by one W-211 pump operating at its lowest allowable speed of $700 \mathrm{rpm}$ for 24 minutes, a degassing run of the same speed and duration would not produce unacceptably large gas releases in AN-105.

\subsubsection{Total Gas Release}

In the current study, we revisited the SY-101 pump testing data with a somewhat more detailed approach. The SY-101 pump runs that were most pertinent to initial degassing operations are those toward the beginning of Phase B, when high-speed runs were aimed at waste regions that were as yet undisturbed or minimally disturbed by pumping. Prior disturbance by GREs cannot be ruled out. Eight such runs were identified and are listed in Table 4.1. The jet discharge velocity $\mathrm{U}_{0}$ was determined from the pump speed by using the pump performance correlation in Figure 2.7 of Caley et al. (1998) and a nozzle diameter $\mathrm{d}_{0}$ of $0.066 \mathrm{~m}$ (2.6 in.).

The exponential gas release model (Hedengren et al. 2000) discussed in Section 2.3 in the analysis of historical gas release data was used to examine the behavior of gas releases during the selected pump runs listed in Table 4.1. This model was originally developed for analysis of spontaneous gas releases in the waste tanks, where the stimulus for an event is not generally known. Examination of hydrogen concentration histories in the headspace of SY-101 for days with pump runs, however, reveals that the induced gas releases exhibit behavior similar to spontaneous releases (see Sections 2.3 and 4.1.2.2).

The general shape of a plot of the hydrogen concentration data versus time for the days of interest shows a characteristic steep rise to a relatively sharp peak at the initiation of the pump run followed by an approximately exponential decay (subsection 4.1.2.2). In many cases, there appear to be secondary releases in the hours following the pump run, similar to the multiple releases discussed in Section 2.3. These releases may be part of the response of the waste to the disturbance due to the pump run, or they may in some cases be induced BDGREs. In either case, the individual gas releases making up the GRE have essentially the same character and are analyzed using the exponential gas release model. The analysis evaluated the hydrogen concentration data for the entire day for each pump run, using the multiple-release analysis approach described in Section 2.3. This analysis was performed for the eight pump runs. 
Table 4.1. SY-101 Pump Runs That Disturbed Fresh Waste

\begin{tabular}{|c|c|c|c|c|c|}
\hline Date & $\begin{array}{l}\text { Direction } \\
\text { (degrees) }\end{array}$ & $\begin{array}{l}\text { Pump } \\
\text { Speed } \\
\text { (rpm) }\end{array}$ & $\begin{array}{c}\text { Run } \\
\text { Duration } \\
\text { (minutes) }\end{array}$ & $\mathbf{U}_{0}(\mathrm{~m} / \mathrm{s})$ & Comment \\
\hline $7 / 26 / 93$ & $0 / 180$ & 997 & 9.3 & 21.1 & $\begin{array}{l}\text { More than } 20 \text { pump runs in this direction } \\
\text { in the preceding } 22 \text { days, but most of these } \\
\text { were less than a minute long or were at } \\
719 \mathrm{rpm} \text { or less. Considering the greater } \\
\text { speed and duration of this run, it is } \\
\text { expected to have disturbed fresh waste. }\end{array}$ \\
\hline $10 / 22 / 93$ & $35 / 215$ & 509 & 19.7 & 11.0 & $\begin{array}{l}\text { One run in this direction the day before, a } \\
\text { run at } 36 \text { and } 216 \text { degrees that lasted less } \\
\text { than a minute. }\end{array}$ \\
\hline $11 / 5 / 93$ & $65 / 245$ & $\begin{array}{l}359 \\
509 \\
719 \\
919\end{array}$ & $\begin{array}{l}19.7 \\
19.7 \\
19.7 \\
19.7\end{array}$ & $\begin{array}{c}7.9 \\
11.0 \\
15.3 \\
19.6\end{array}$ & No earlier runs in this direction. \\
\hline $11 / 10 / 93$ & $95 / 275$ & 359 & 20 & 7.9 & No earlier runs in this direction. \\
\hline $11 / 11 / 93$ & $95 / 275$ & $\begin{array}{l}359 \\
509 \\
719 \\
919 \\
\end{array}$ & $\begin{array}{l}19.7 \\
19.7 \\
19.7 \\
19.7 \\
\end{array}$ & $\begin{array}{c}7.9 \\
11.0 \\
15.3 \\
19.6 \\
\end{array}$ & $\begin{array}{l}\text { The run on the previous day would have } \\
\text { disturbed a small amount of waste in this } \\
\text { direction, but much fresh waste must have } \\
\text { been reached by this run. }\end{array}$ \\
\hline $11 / 12 / 93$ & $125 / 305$ & $\begin{array}{l}359 \\
509 \\
719 \\
919\end{array}$ & $\begin{array}{l}19.7 \\
19.7 \\
19.7 \\
19.7\end{array}$ & $\begin{array}{c}7.9 \\
11.0 \\
15.3 \\
19.6\end{array}$ & No earlier runs in this direction. \\
\hline $11 / 13 / 93$ & $155 / 335$ & $\begin{array}{l}359 \\
509 \\
719 \\
919 \\
\end{array}$ & $\begin{array}{l}19.7 \\
19.7 \\
19.7 \\
19.7\end{array}$ & $\begin{array}{c}7.9 \\
11.0 \\
15.3 \\
19.6 \\
\end{array}$ & No earlier runs in this direction. \\
\hline $11 / 21 / 93$ & $125 / 305$ & 749 & 60 & 15.9 & $\begin{array}{l}\text { Although the ramp-up run of } 11 / 12 / 93 \text { had } \\
\text { been in this direction, this run was also } \\
\text { included as a nonramp-up run for } \\
\text { comparison. }\end{array}$ \\
\hline
\end{tabular}

Table 4.2 presents the total gas release volume for the respective pump runs as well as results for a selected subset of the data consisting of only the initial gas release event at the beginning of the pump run. The initial gas release or the first peak of the data is relevant to the analyses presented in Sections 4.2 through 4.6.

From the point of view of establishing a relationship between pump run parameters and releases from undisturbed waste, the full data set from Phases A and B is somewhat ambiguous. Large releases occurred on the days listed in Table 4.1 but also on days when pump runs were not carried out, or when there were only short low-speed runs, or when the jets were directed into waste that had already been disturbed.

The arguments developed in Section 4.1.1 from theoretical consideration of jet mixing behavior suggest that the quantities $\rho_{0}\left(\mathrm{U}_{0} \mathrm{~d}_{0}\right)^{2}, \mathrm{tU}_{0} \mathrm{~d}_{0}{ }^{2}$, and $\mathrm{t} \rho_{0} \mathrm{U}_{0}{ }^{3} \mathrm{~d}_{0}{ }^{2}$ (corresponding to discharge momentum, volume, and power, respectively) could be related to the volume of waste disturbed by the pump jets. Figures 4.1 to 4.3 show the daily gas release volume (in excess of background) 
Table 4.2. Gas Releases Associated with SY-101 Pump Runs of Interest

\begin{tabular}{|c|c|c|c||}
\hline Pump & Total Gas & \multicolumn{2}{|c|}{ Initial Gas Release } \\
\cline { 3 - 4 } & $\begin{array}{c}\text { Rute } \\
\text { Release }\end{array}$ & $\begin{array}{c}\text { Total Gas Release } \\
\left(\mathbf{m}^{\mathbf{3}}\right)\end{array}$ & $\begin{array}{c}\text { Time to Peak Gas Release Rate } \\
(\mathbf{m i n})\end{array}$ \\
\hline $7 / 26 / 93$ & 1.73 & 1.16 & 6.0 \\
\hline $10 / 22 / 93$ & 0.39 & 0.39 & 20.0 \\
\hline $11 / 5 / 93$ & 3.62 & 2.71 & 38.0 \\
\hline $11 / 10 / 93$ & 0.14 & 0.14 & 17.9 \\
\hline $11 / 11 / 93$ & 4.13 & 1.61 & 20.0 \\
\hline $11 / 12 / 93$ & 5.07 & 2.82 & 29.0 \\
\hline $11 / 13 / 93$ & 4.83 & 2.41 & 20.2 \\
\hline $11 / 21 / 93$ & 3.41 & 1.12 & 32.4 \\
\hline
\end{tabular}

plotted versus these three parameters (the waste density has not been included to keep it simple). In the cases of the ramp-up runs on November 5, 11, and 13, 1993, the values used were the weighted averages of each incremental step.

In Figure 4.1 we see little correlation with discharge momentum, evidently because run time has not been taken into account. However, Figures 4.2 and 4.3 show the consistent trend of increasing gas release volume for the quantities $\mathrm{tU}_{0} \mathrm{~d}_{0}{ }^{2}$ and $\mathrm{tU}_{0}{ }^{3} \mathrm{~d}_{0}{ }^{2}$, although there is scatter at the higher values. From the limited data available, parameter groups associated with volume flow and power are fairly equivalent in terms of how they tie in with gas release.

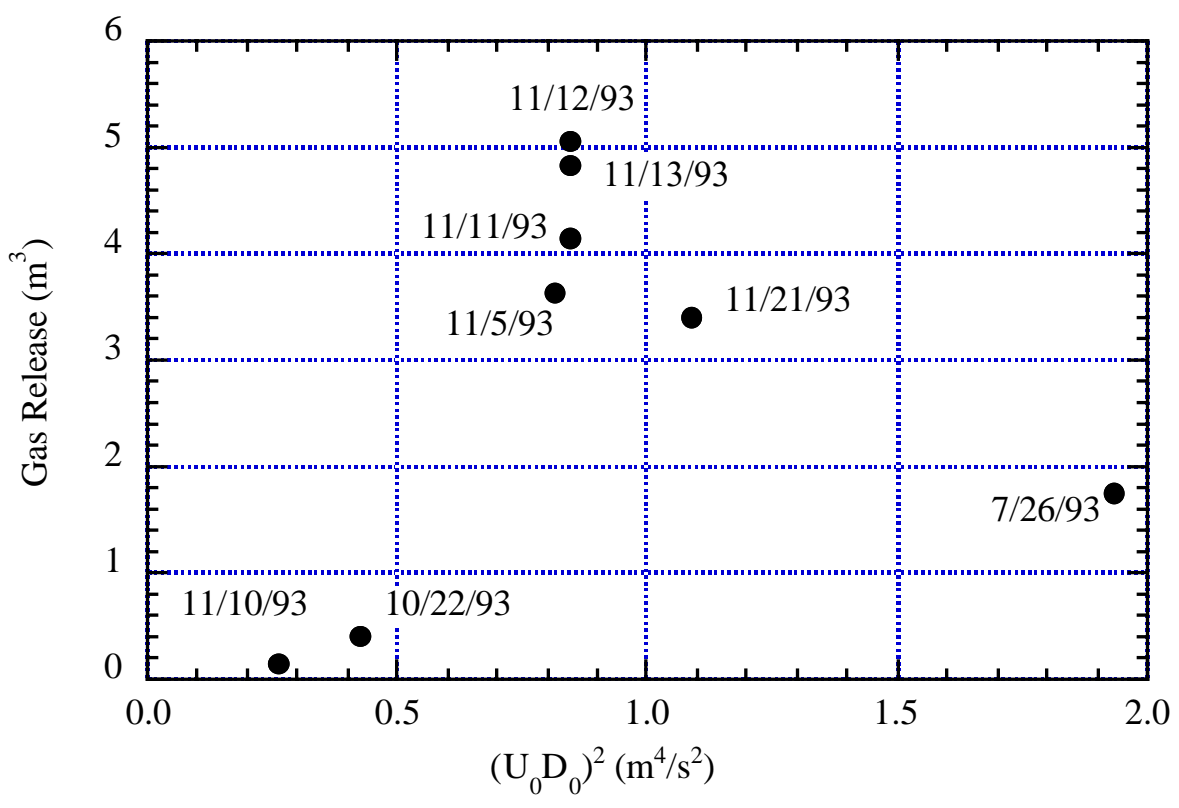

Figure 4.1. Gas Release from SY-101 Mixer-Pump Operation Correlated with Jet Momentum Scaling 


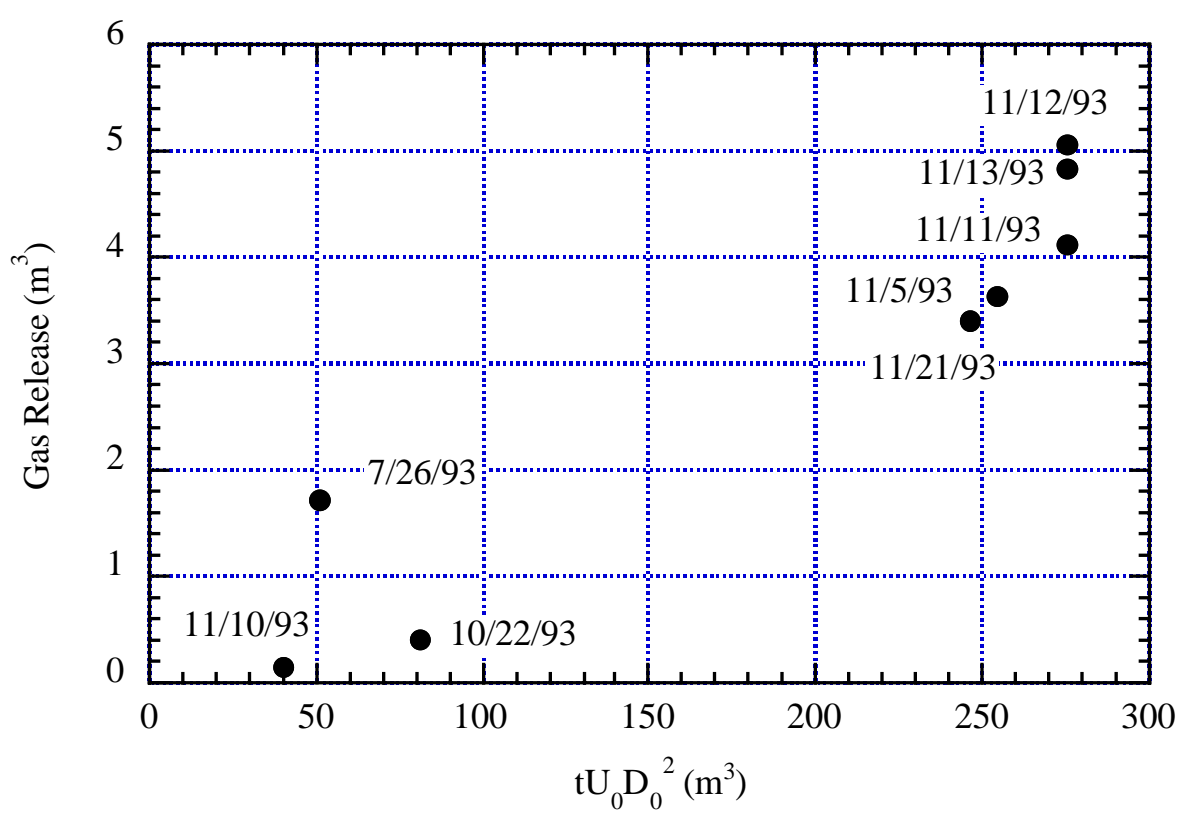

Figure 4.2. Gas Release from SY-101 Mixer-Pump Operation Correlated with Jet Volume Flow Scaling

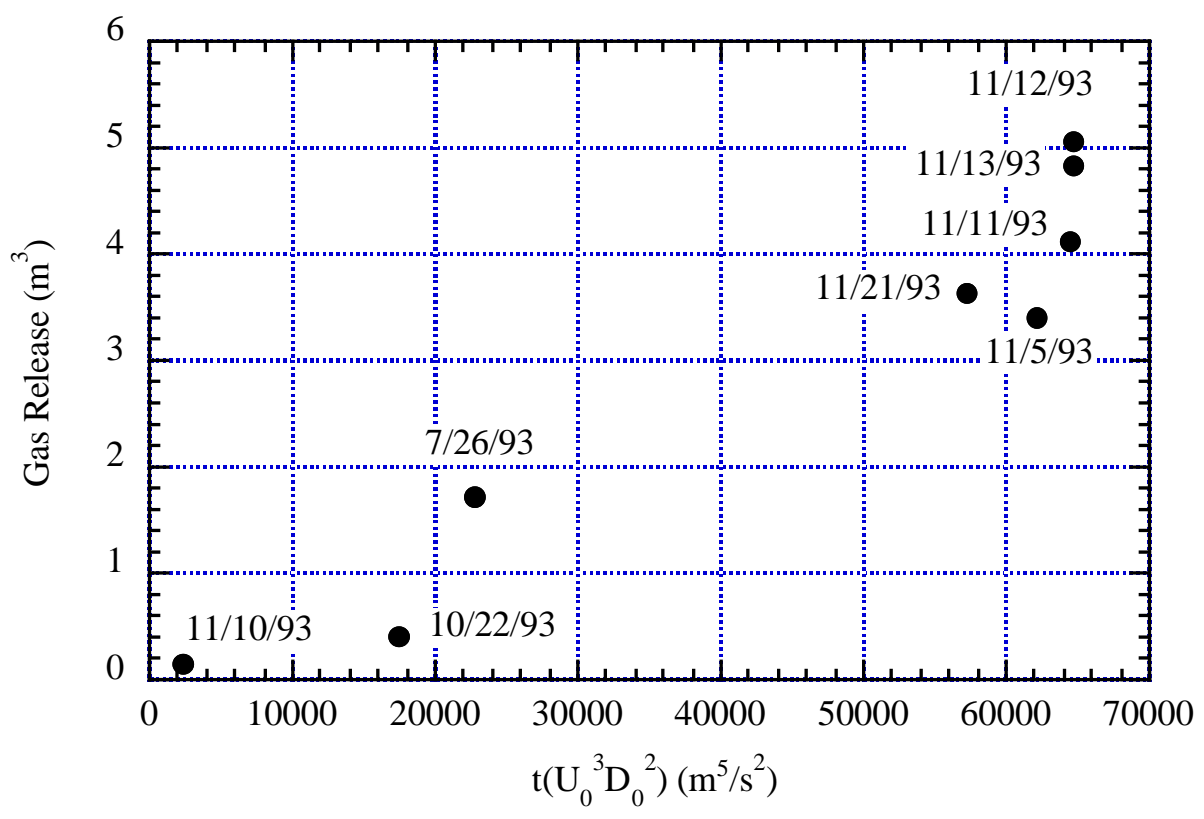

Figure 4.3. Gas Release from SY-101 Mixer-Pump Operation Correlated with Jet Hydraulic Power Scaling 


\subsubsection{Gas Release Delay}

Another perspective on the relationship between the pump run duration and gas release was found by examining the time profiles of the headspace hydrogen for the days of interest. These profiles are shown in Figures 4.4 through 4.11. All of the data indicate that time to peak gas release from the start of the pump run exceeds pump run time. Figure 4.12 shows that the time to peak gas release increases with the gas release volume. While the reason for these trends cannot be known precisely, the behavior is consistent with a gas holdup mechanism the crust might provide. Figures 4.13 and 4.14 show gas release delay plotted versus the volume flow, $\mathrm{tU}_{0} \mathrm{~d}_{0}{ }^{2}$, and the jet power, $\mathrm{tU}_{0}{ }^{3} \mathrm{~d}_{0}{ }^{2}$. Again, a reasonable relationship can be seen for both scaling groups, with little indication of which provides a better correlation.

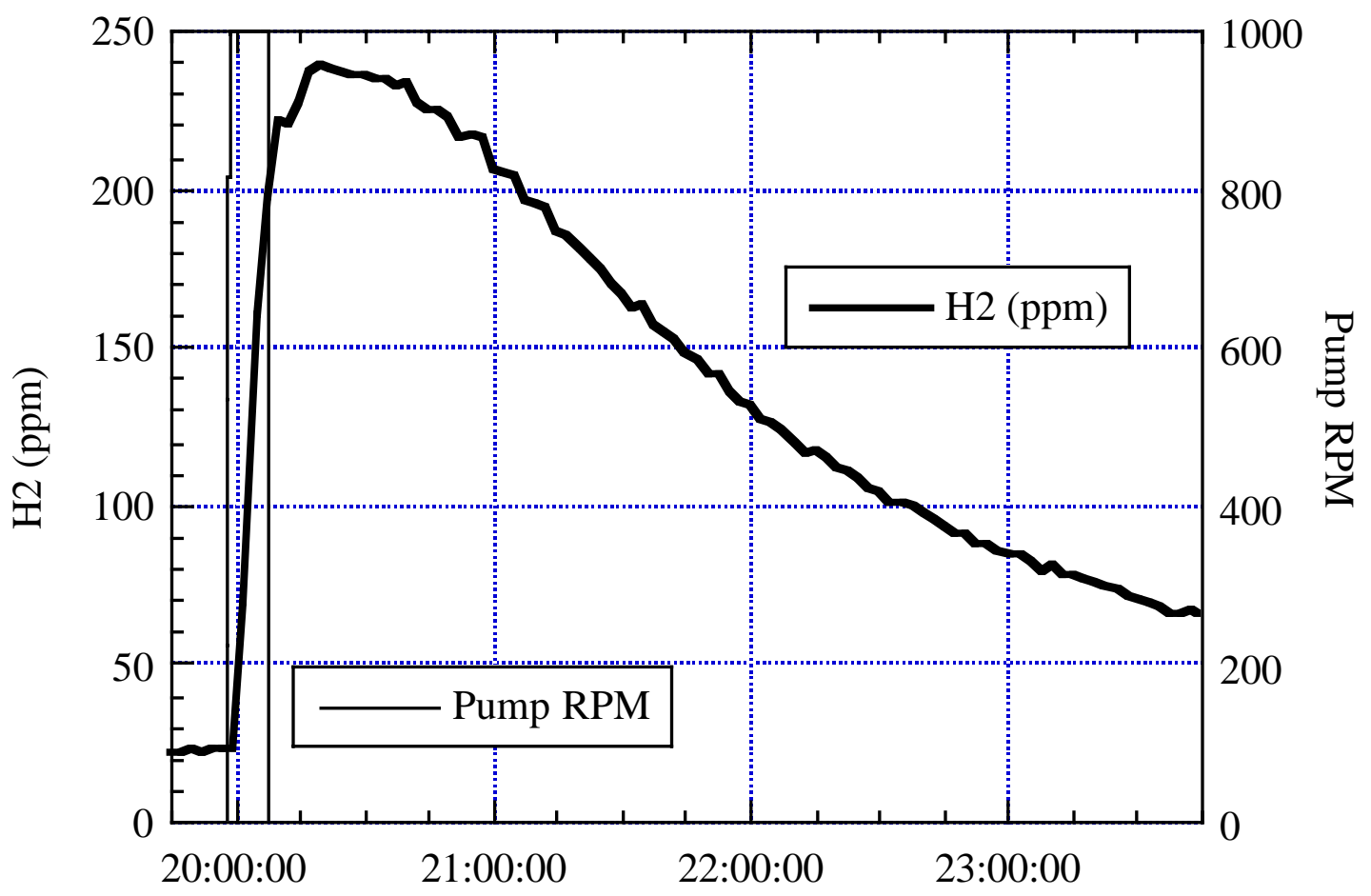

Figure 4.4. July 26, 1993 Pump Run and GC Data 


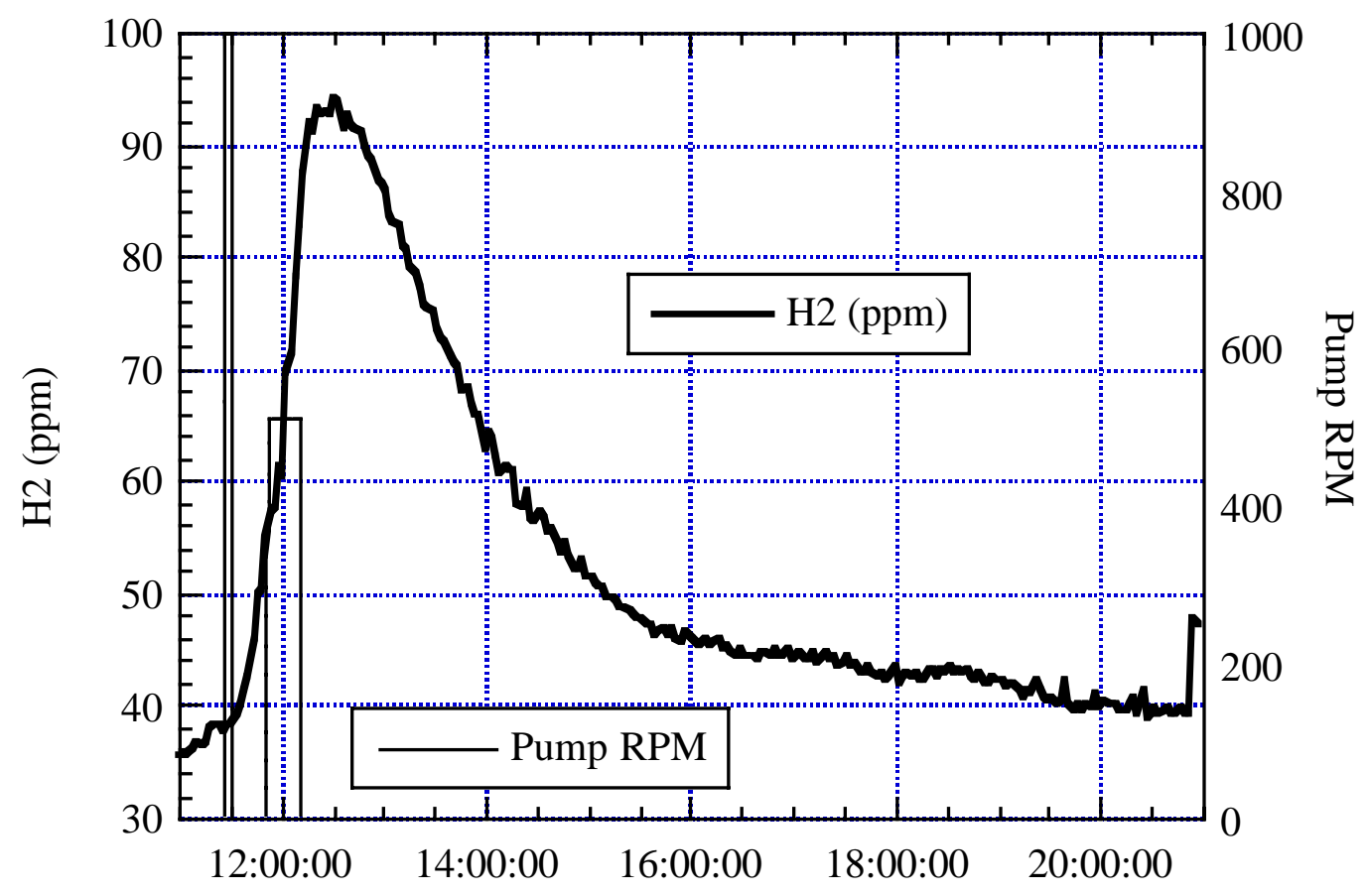

Figure 4.5. October 22, 1993 Pump Run and GC Data

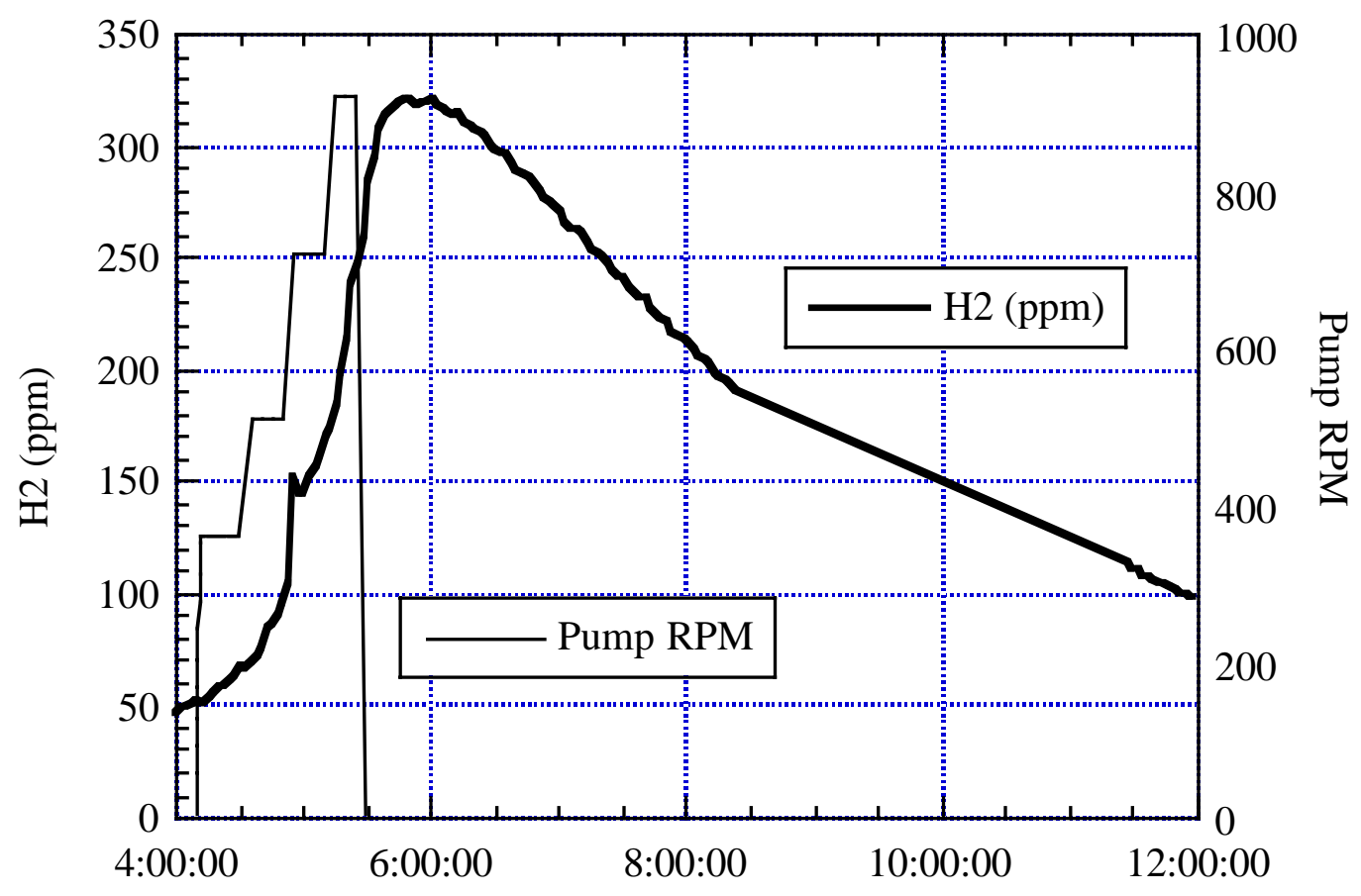

Figure 4.6. November 5, 1993 Pump Run and GC Data 


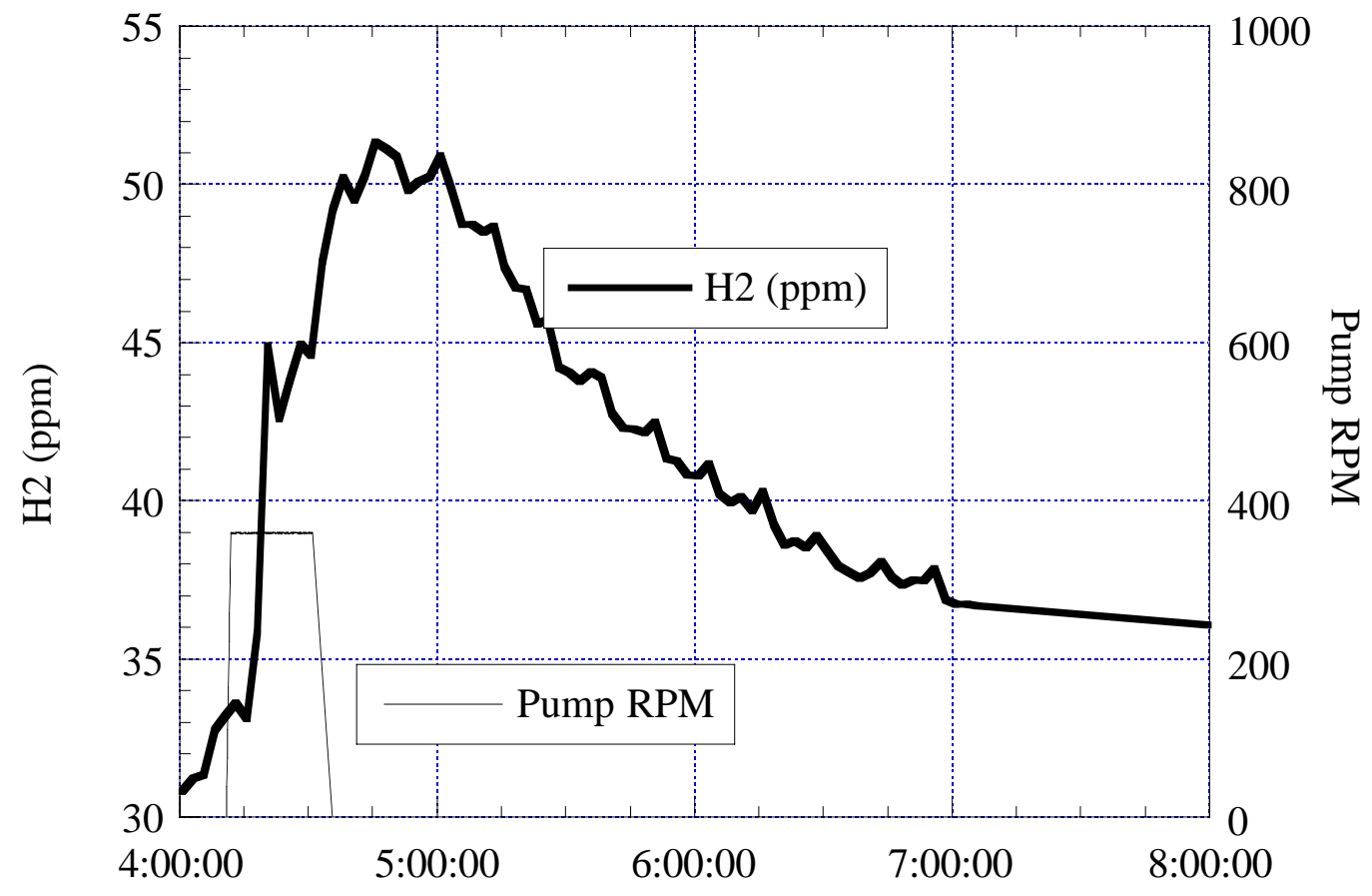

Figure 4.7. November 10, 1993 Pump Run and GC Data

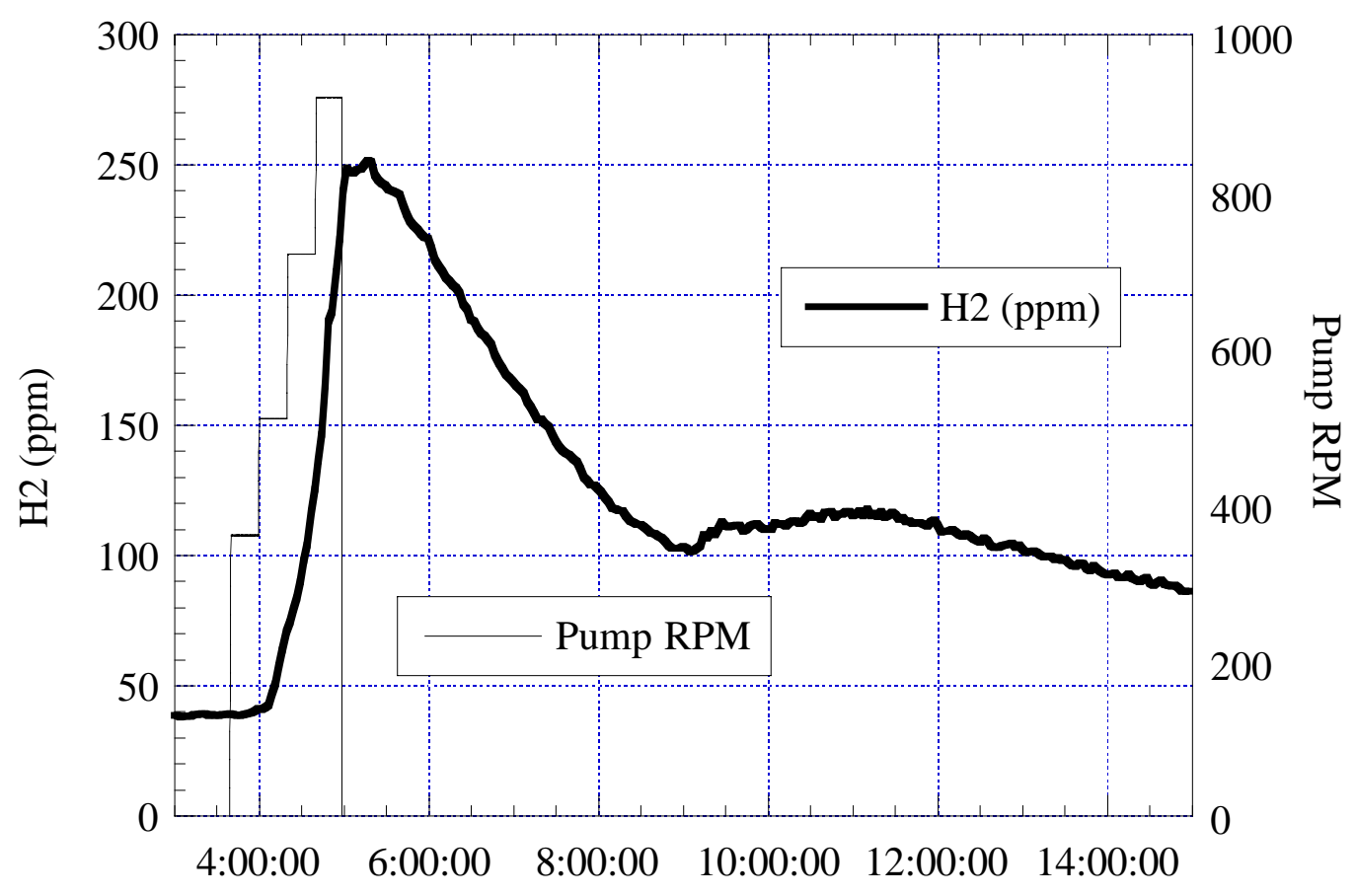

Figure 4.8. November 11, 1993 Pump Run and GC Data 


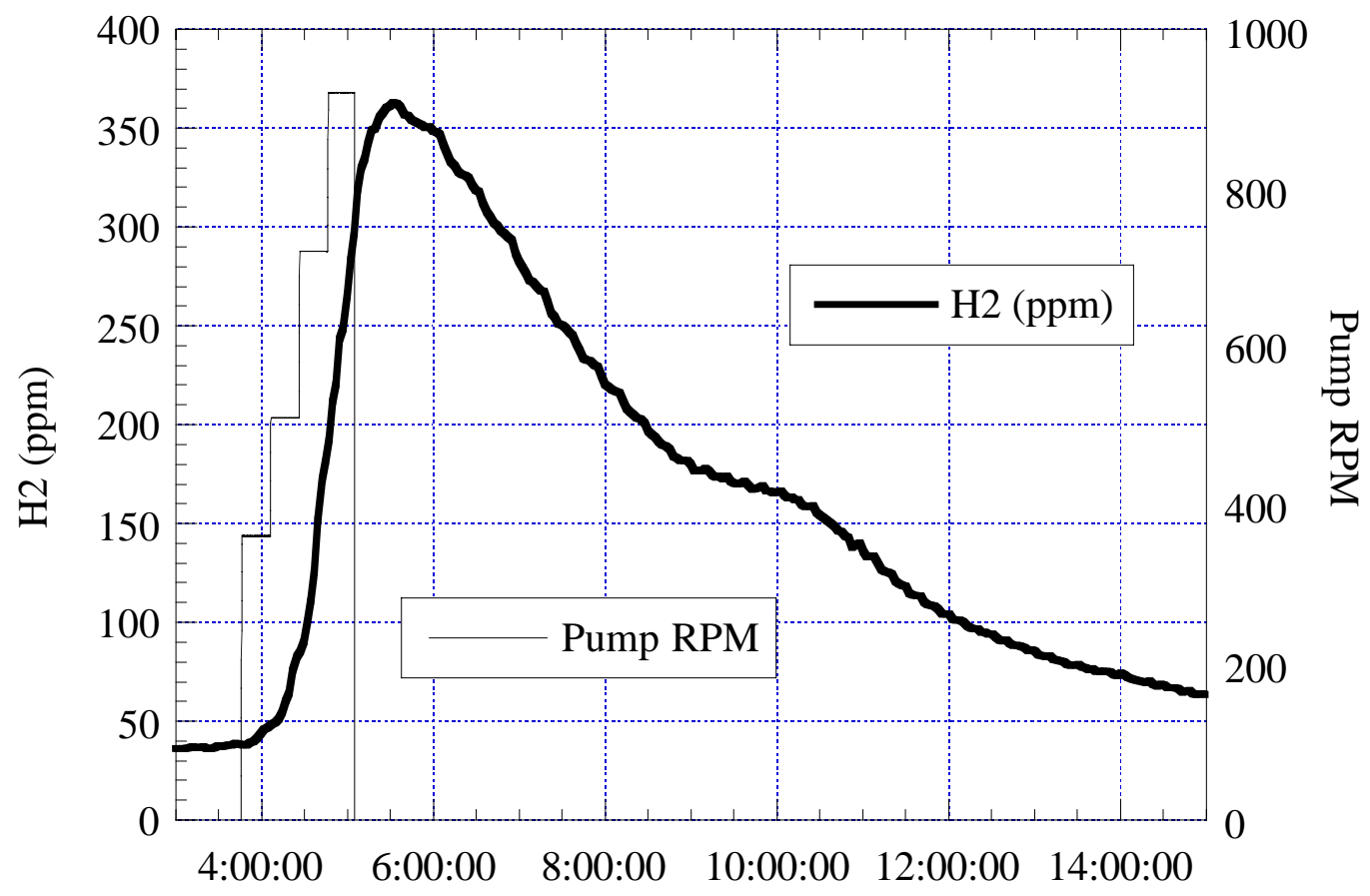

Figure 4.9. November 12, 1993 Pump Run and GC Data

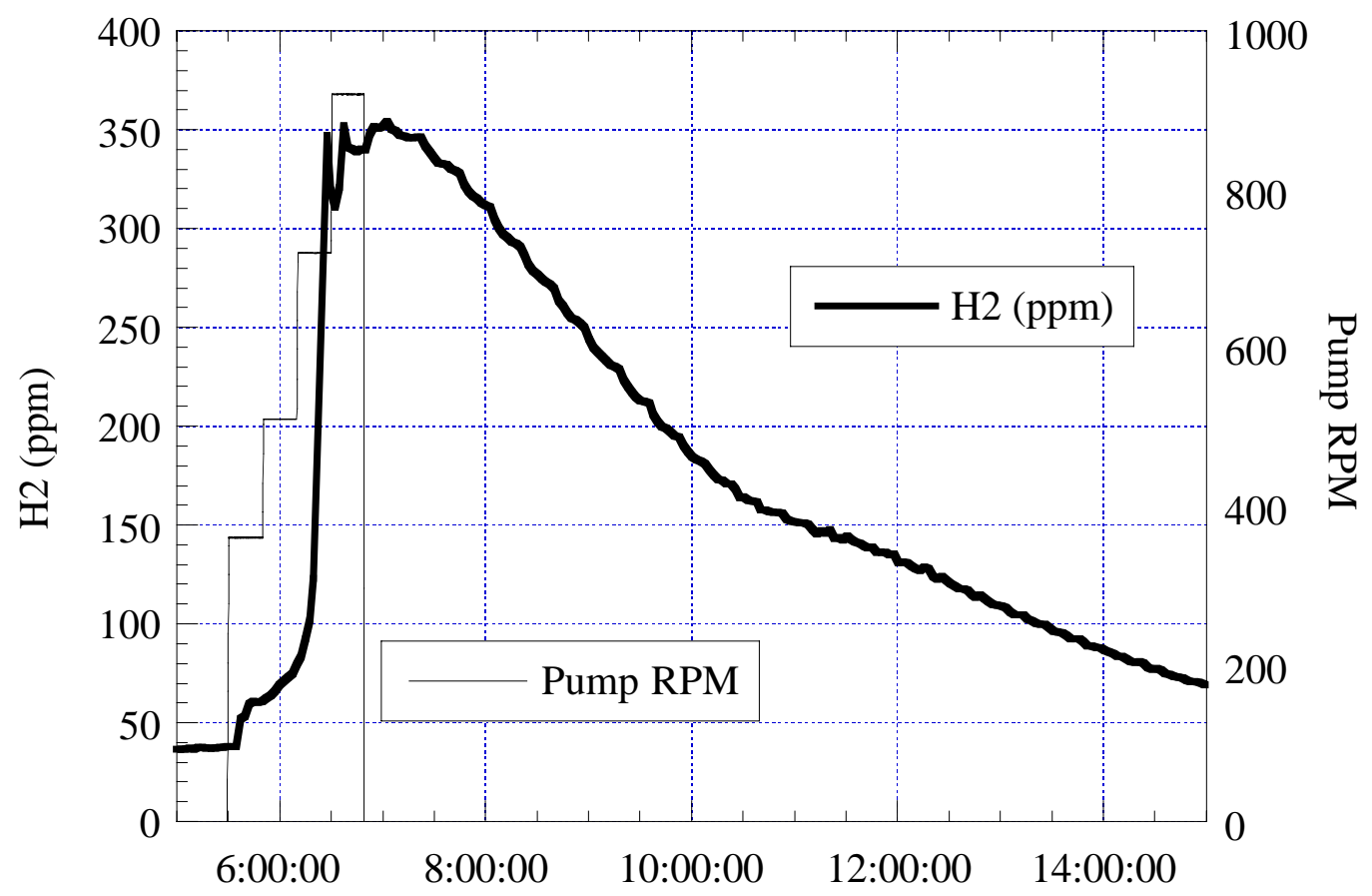

Figure 4.10. November 13, 1993 Pump Run and GC Data 


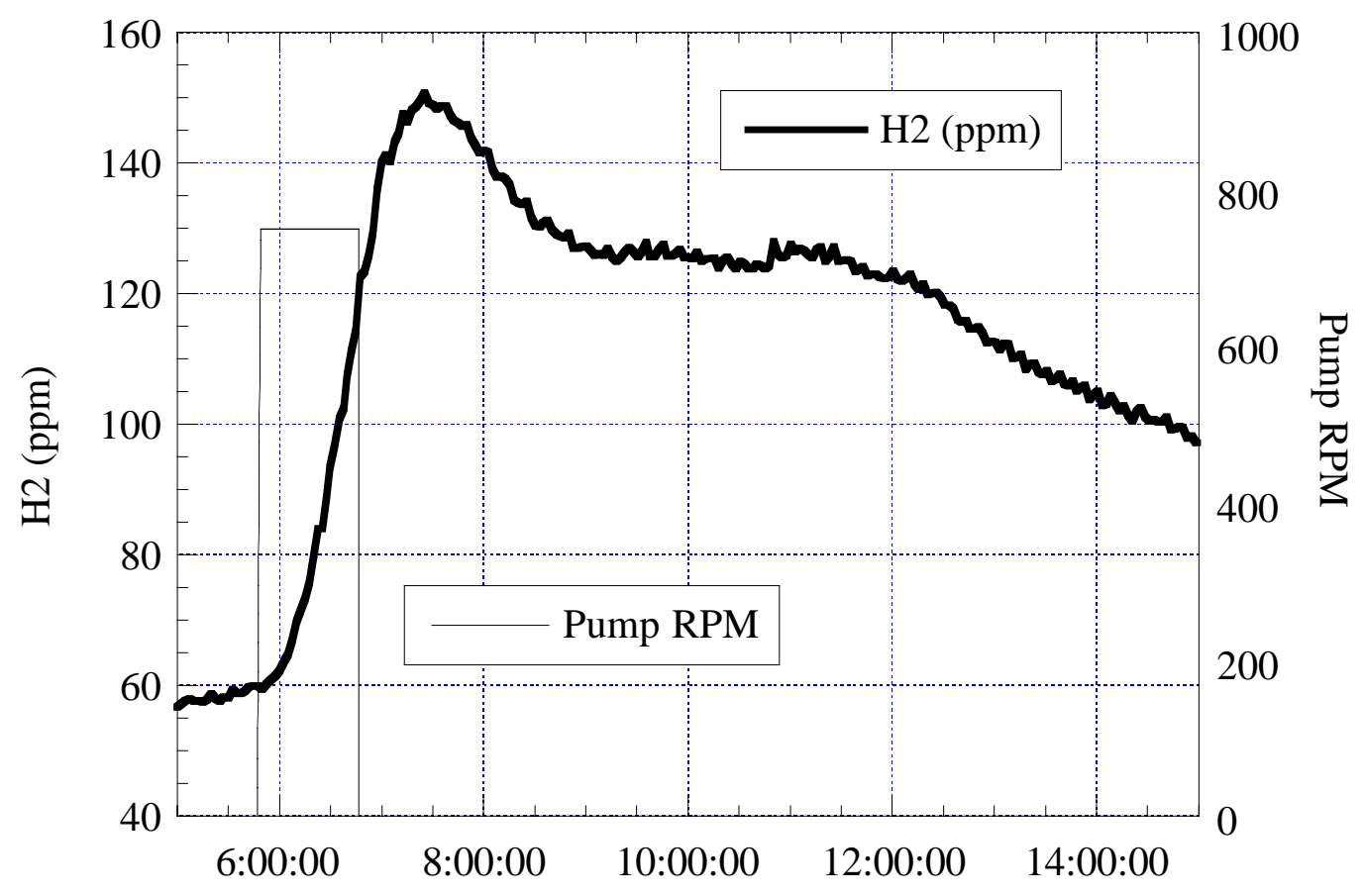

Figure 4.11. November 21, 1993 Pump Run and GC Data

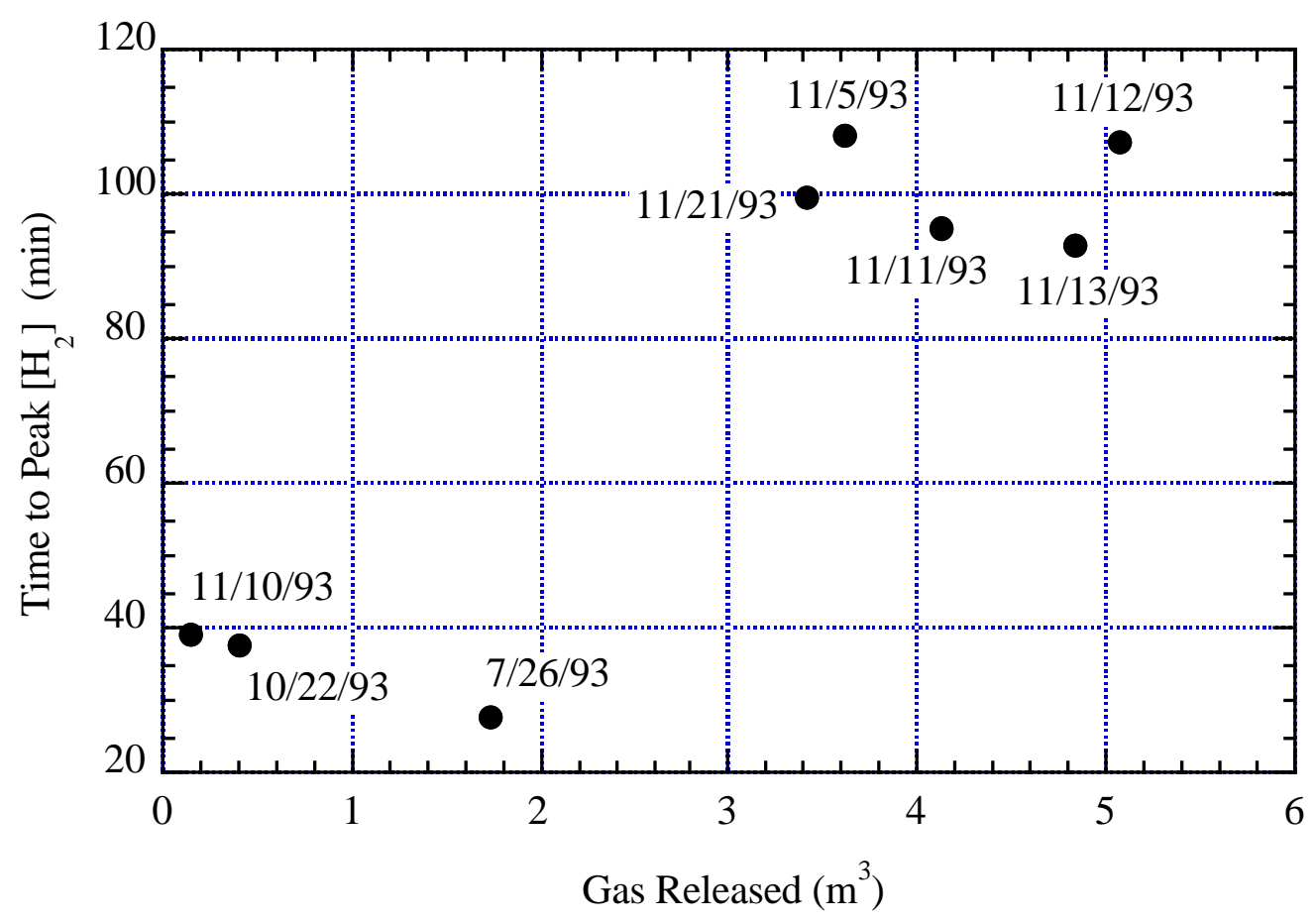

Figure 4.12. Relation Between Peak $\mathrm{H}_{2}$ Delay and Total Gas Release 


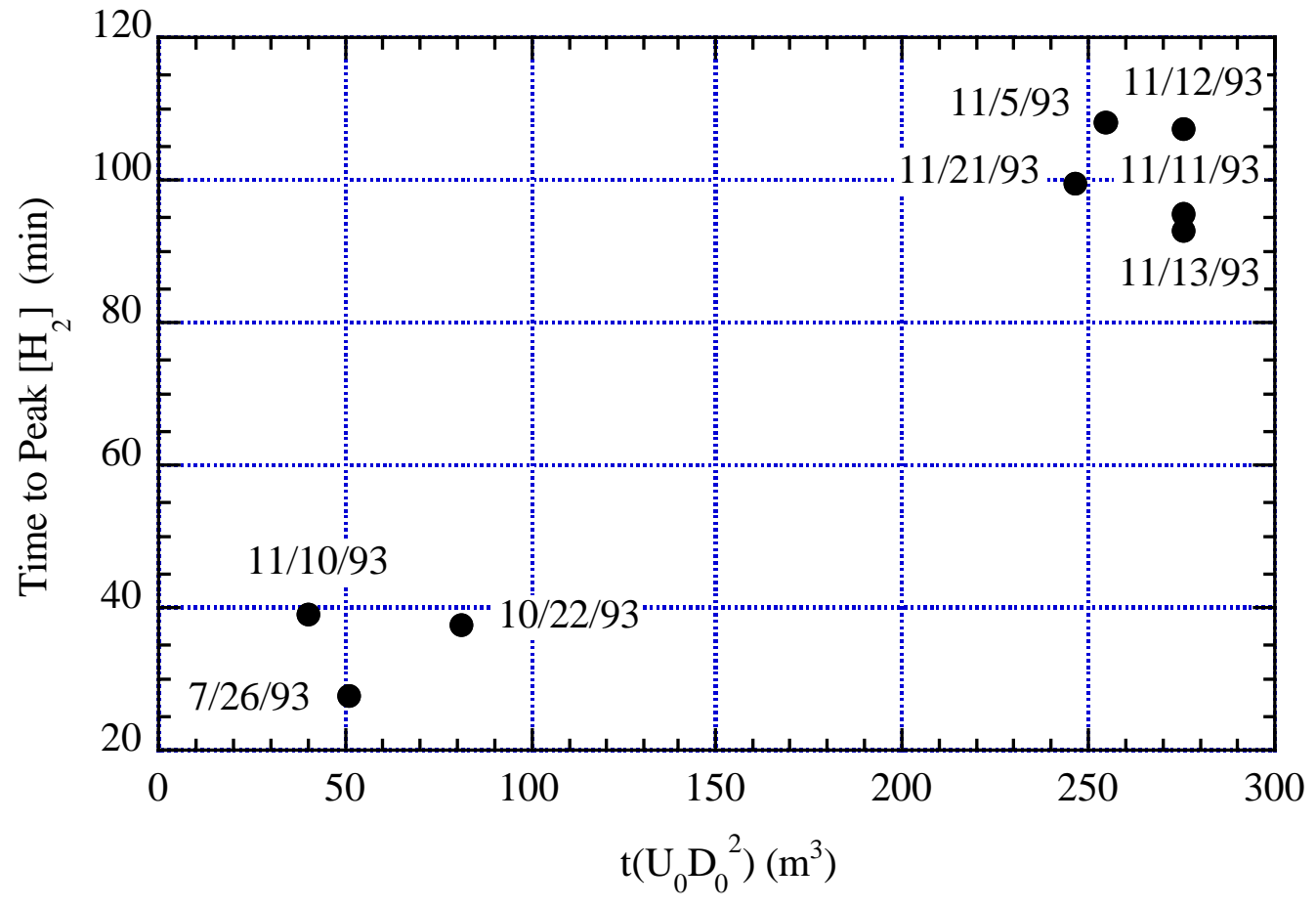

Figure 4.13. Relation Between Peak $\mathrm{H}_{2}$ Delay and Jet Flow Rate Scaling

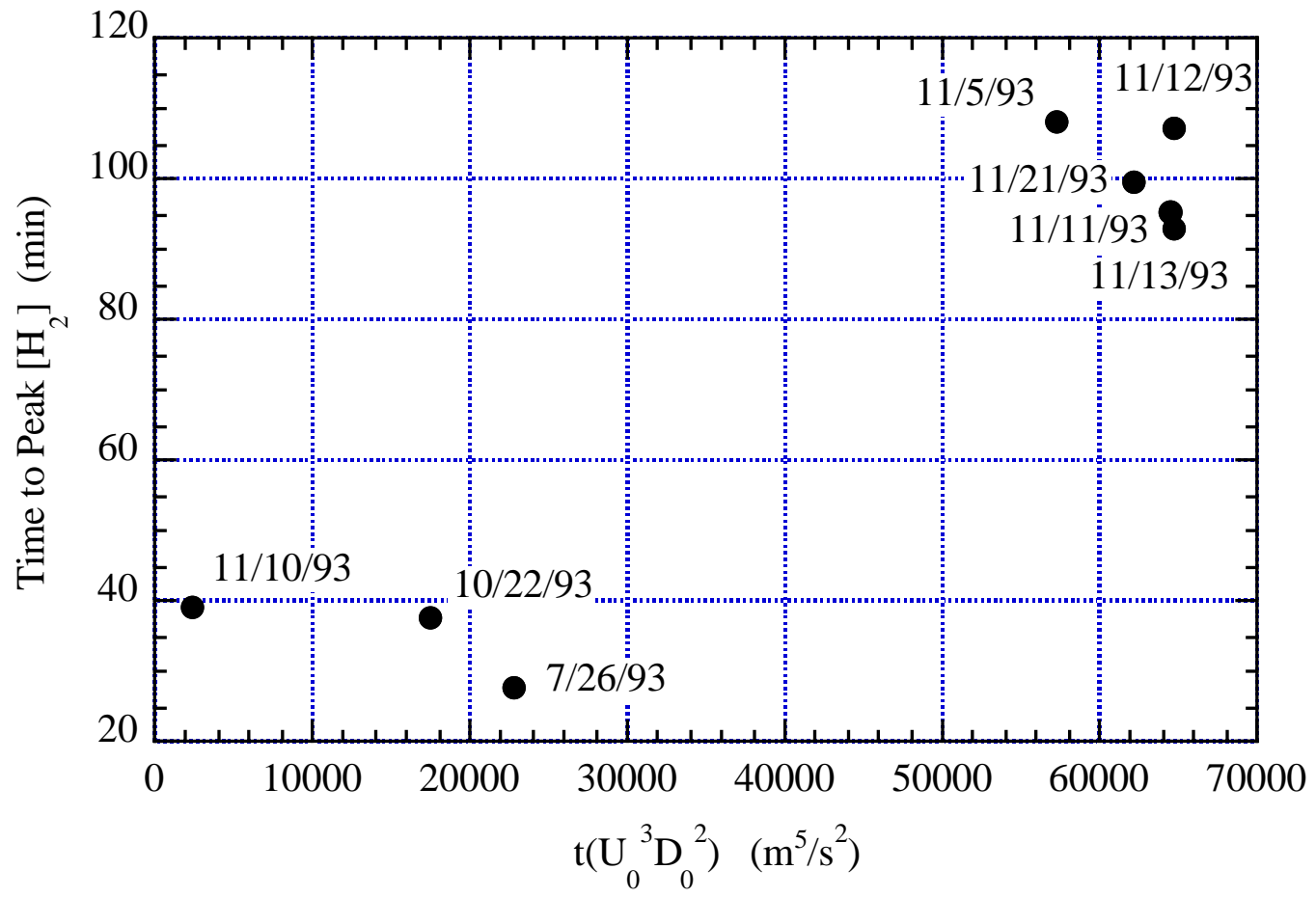

Figure 4.14. Relation Between Peak $\mathrm{H}_{2}$ Delay and Jet Hydraulic Power Scaling 


\subsubsection{Energy Balance Model for Waste Disturbance}

We now attempt to develop a model for determining the gas release as a function of mixer pump parameters and waste properties.

\subsubsection{The Free Turbulent Jet}

We begin by considering the behavior of free, nonbuoyant turbulent jets in Newtonian fluids. While the jet created by the mixer pump is not free (the unmobilized nonconvective layer may act as a solid surface) and may not be Newtonian, some of the basic relations and scaling may still be applicable. Figure 4.15 shows the basic features of the free jet.

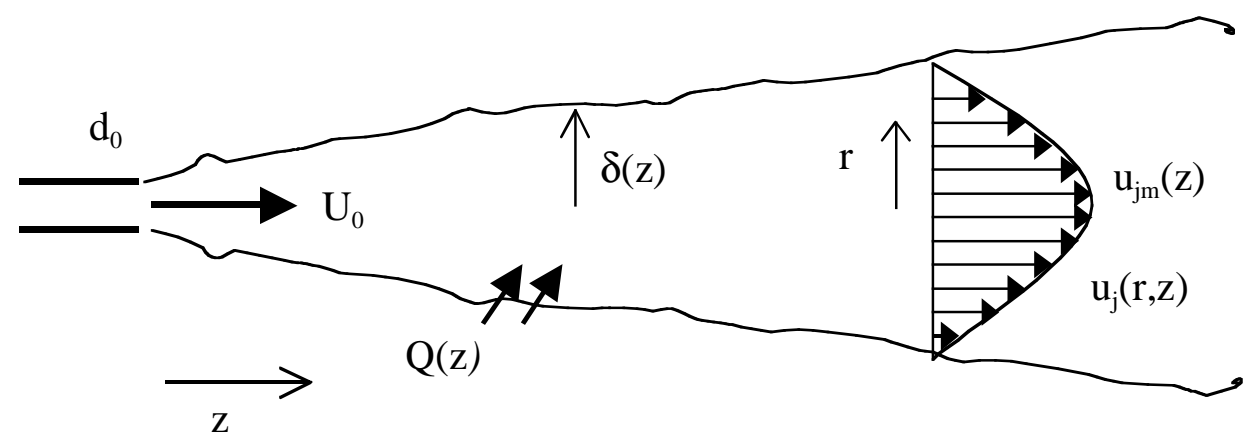

Figure 4.15. The Free Turbulent Jet

The jet has a time-averaged velocity profile that varies in the both the $\mathrm{z}$ and $\mathrm{r}$ directions. The peak velocity, $\mathrm{u}_{\mathrm{jm}}$, varies according to

$$
\mathrm{u}_{\mathrm{jm}}(\mathrm{z})=\frac{6 \mathrm{U}_{0} \mathrm{~d}_{0}}{\mathrm{z}}
$$

The radial variation can be approximated by a Gaussian:

$$
u_{j}(r, z)=u_{j m}(z) e^{-4.6\left(\frac{r}{\delta}\right)^{2}}
$$

where $\delta$ is the half jet spread angle. The average jet velocity at any z location is approximately given by

$$
\overline{\mathrm{u}}_{\mathrm{j}}(\mathrm{z})=\frac{\mathrm{u}_{\mathrm{jm}}(\mathrm{z})}{\delta^{2}(\mathrm{z})} \int_{0}^{\infty} \mathrm{re}^{-4.6\left(\frac{\mathrm{r}}{\delta(\mathrm{z})}\right)^{2}} \mathrm{dr}
$$

which can be evaluated to give

$$
\overline{\mathrm{u}}_{\mathrm{j}}(\mathrm{z}) \approx 0.11 \mathrm{u}_{\mathrm{jm}}(\mathrm{z})
$$


The jet spreads at a constant half-angle $\theta$ so that

$$
\delta(\mathrm{z})=\theta \mathrm{z}
$$

Turbulent jets spread at about 15 degrees total angle, corresponding to $\theta=0.13 \mathrm{rad}$. The swept volume of the jet cone is given by

$$
\mathrm{V}(\mathrm{z})=\frac{\pi}{3} \delta^{2} \mathrm{z}=\frac{\pi}{3} \theta^{2} \mathrm{z}^{3}
$$

Finally, the total entrainment rate into the jet up to location $\mathrm{z}$ is given by (Rajaratnam 1974):

$$
\mathrm{Q}(\mathrm{z}) \approx 0.3 \mathrm{Q}_{0} \mathrm{z} / \mathrm{d}_{0}
$$

where $\mathrm{Q}_{0}$ is the discharge flow rate given by Eq. (4.4)

Finally, consider the efficiency of the jet from a hydraulic power point of view. The initial power of the jet at the nozzle is given by Eq. (4.8). The power at any station $\mathrm{z}$ is given by

$$
\overline{\mathrm{p}}_{\mathrm{j}}(\mathrm{z})=\frac{1}{2} \rho \mathrm{u}^{3}{ }_{\mathrm{jm}}(\mathrm{z}) \int_{0}^{\infty} \mathrm{r} \mathrm{e}^{-3 \cdot 4.6\left(\frac{\mathrm{r}}{\delta(\mathrm{z})}\right)^{2}} \mathrm{dr}
$$

which is evaluated to give

$$
\overline{\dot{p}}_{j}(z)=\frac{0.036}{2} \rho u^{3}{ }_{j m}(z) \delta^{2}
$$

Here we see that the average power at any location is only about $3.6 \%$ of the power computed from maximum velocity at the same location. This power is very much less than the initial power of the jet as it leaves the nozzle. Hence it is evident that a great deal of energy is dissipated in the turbulent jet, and only a small fraction of the initial energy is available for yielding the sediment layer material.

\subsubsection{A Model for Jet Penetration in a Pseudo Solid}

Now we consider the case of a turbulent jet penetrating into a weak pseudo solid such as the sediment layer. Consider a conceptual model for the penetration, as shown in Figure 4.16. Here the jet has a distinct solid front with area $A_{f}$ and is moving at some average velocity $u_{f}$ into the pseudo solid with strength $\tau$. Well behind the front, we assume the jet looks similar to the free turbulent jet. The spread angle and the entrainment may be different, but we assume the functional forms in Eq. (4.17) to (4.23) still hold, possibly with modified constants. If the front velocity $u_{f}$ is smaller than the equivalent free jet velocity $u_{j}$ that would be present at the same location, the fluid must turn outward radially or return toward the nozzle to satisfy continuity. 


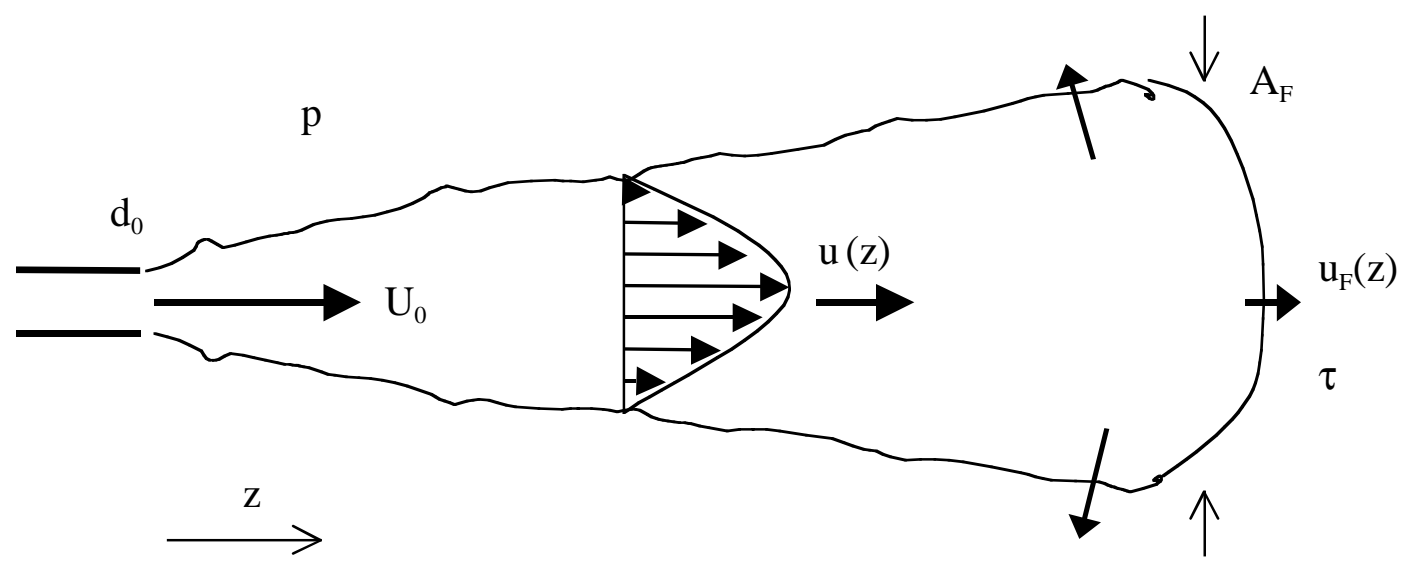

Figure 4.16. A Model for Jet Penetration into a Pseudo Solid

\subsubsection{An Energy Balance}

We now attempt to develop and expression for the movement of the jet front. If this can be done, the swept volume can be determined as a function of time, and the associated gas release can be computed.

Consider an energy balance on the moving front:

$$
\begin{aligned}
& \text { Rate of work } \\
& \text { applied to front }
\end{aligned}=\begin{aligned}
& \text { Power required } \\
& \text { to yield solid }
\end{aligned}-\begin{aligned}
& \text { Losses due to } \\
& \text { dissipation }
\end{aligned}+\begin{aligned}
& \text { Hydraulic power } \\
& \text { of fluid at moving } \\
& \text { front }
\end{aligned}
$$

This is expressed mathematically by

$$
\left(\mathrm{p}_{\mathrm{f}}-\mathrm{p}_{0}\right) \mathrm{A}_{\mathrm{f}} \mathrm{u}_{\mathrm{f}}=\tau \mathrm{A}_{\mathrm{f}} \mathrm{u}_{\mathrm{f}}+\Phi+\frac{1}{2} \rho \mathrm{u}_{\mathrm{f}}^{2} \mathrm{~A}_{\mathrm{f}} \mathrm{u}_{\mathrm{f}}
$$

where $\mathrm{p}_{\mathrm{f}}$ is the local pressure at the front and $\mathrm{p}_{0}$ is the ambient pressure in the sediment layer at the elevation of the jet. The total dissipation $\Phi$ takes into account all viscous loss mechanisms. We assume the dissipation is proportional to the net work on the front so that

$$
\Phi=\mathrm{c}\left(\mathrm{p}_{\mathrm{f}}-\mathrm{p}_{0}-\tau\right) \mathrm{A}_{\mathrm{f}} \mathrm{u}_{\mathrm{f}}
$$

So Eq. (4.2) can be written

$$
\frac{1}{2} \rho u_{\mathrm{f}}^{2}=\mathrm{e}\left(\mathrm{p}_{\mathrm{f}}-\mathrm{p}_{0}-\tau\right)
$$

where $\mathrm{e}=1-\mathrm{c}$ is the efficiency of the process. When $\mathrm{c}=0$ (no dissipation), $\mathrm{e}=1$, and from Eq. (4.28) we see that all the net pressure work is converted into kinetic energy of the front. 
The total pressure $\mathrm{p}_{\mathrm{tf}}$ at the front is given by

$$
\mathrm{p}_{\mathrm{tf}}=\mathrm{p}_{\mathrm{f}}+\frac{1}{2} \rho \mathrm{u}_{\mathrm{f}}^{2}
$$

The total pressure, $\mathrm{p}_{\mathrm{tf}}$, will be approximately equal to the total pressure that would be present at the same location for a free turbulent jet, $\mathrm{p}_{\mathrm{t} j}$. This is indicated from experimental data for free jets impinging on solid walls (Rajaratnam 1974). Therefore, we can write

$$
\mathrm{p}_{\mathrm{f}}+\frac{1}{2} \rho \mathrm{u}_{\mathrm{f}}^{2} \approx \mathrm{p}_{0}+\frac{1}{2} \rho \mathrm{u}_{\mathrm{jm}}^{2}
$$

We expect that the motion of the front is slow enough so that the dynamic component in Eq. (4.30) is negligible compared to the static component. With this approximation we get

$$
\mathrm{p}_{\mathrm{f}}-\mathrm{p}_{0} \approx \frac{1}{2} \rho \mathrm{u}_{\mathrm{jm}}^{2}
$$

The pressure term in Eq. (4.28) can be evaluated with the aid of Eq. (4.31) to obtain

$$
\frac{1}{2} \rho u_{\mathrm{f}}^{2}=\mathrm{e}\left(\frac{1}{2} \rho u_{\mathrm{jm}}^{2}-\tau\right)
$$

In the limit of no shear strength, the local efficiency would be 1 (because no yield process would occur), and Eq. (4.32) shows that the front velocity becomes the free jet velocity.

When the free jet velocity given by Eq. (4.17) is substituted into Eq. (4.32), we obtain an expression for the front velocity as a function of position $\mathrm{z}_{\mathrm{f}}$ :

$$
u_{f}\left(z_{f}\right)=U_{0} \sqrt{\left.e\left(\frac{6 d_{0}}{z_{f}}\right)^{2}-\frac{2 \tau}{\rho U_{0}^{2}}\right)}
$$

Noting that $\mathrm{u}_{\mathrm{f}}=\mathrm{dz}_{\mathrm{f}} / \mathrm{dt}$, Eq. (4.33) can be integrated to obtain the front position as a function of time. We can write Eq. (4.33) as

$$
\frac{d z_{f}^{\prime}}{d t^{\prime}}=\sqrt{e\left(\left(\frac{6}{z_{f}^{\prime}}\right)^{2}-\gamma\right)}
$$

where

$$
\begin{aligned}
& \mathrm{z}_{\mathrm{f}}^{\prime}=\mathrm{z}_{\mathrm{f}} / \mathrm{d}_{0} \\
& \mathrm{t}^{\prime}=\mathrm{tU}_{0} / \mathrm{d}_{0}
\end{aligned}
$$




$$
\gamma=\frac{2 \tau}{\rho U_{0}^{2}}
$$

The term $\gamma$ is the ratio of material strength to jet momentum. For $\gamma<<1$, the material is weak compared with the jet's penetrating strength, and we expect significant penetration. For $\gamma \sim 1$, we expect the jet to do very little, or very slow, penetrating. For $\gamma>1$ we expect that the jet will not penetrate into the waste sediment.

Equation (4.34) can be integrated to obtain

$$
\mathrm{z}_{\mathrm{f}}^{\prime}\left(\mathrm{t}^{\prime}\right)=\frac{6}{\sqrt{\gamma}}\left(1-\left(1-\frac{\gamma \sqrt{\mathrm{e}} \mathrm{t}^{\prime}}{6}\right)^{2}\right)^{1 / 2}
$$

The time to steady state (when the front no longer progresses) is

$$
\mathrm{t}_{\max }^{\prime}=\frac{6}{\gamma \sqrt{\mathrm{e}}}
$$

In terms of dimensional variables this is

$$
\begin{aligned}
& \mathrm{z}_{\mathrm{f}}(\mathrm{t})=\frac{6 \mathrm{~d}_{0}}{\sqrt{\gamma}}\left(1-\left(1-\frac{\gamma \sqrt{\mathrm{e}} \mathrm{tU}_{0}}{6 \mathrm{~d}_{0}}\right)^{2}\right)^{1 / 2} \\
& \mathrm{t}_{\max }=\frac{6 \mathrm{~d}_{0}}{\gamma \sqrt{\mathrm{e}} \mathrm{U}_{0}}=\frac{3 \rho}{\tau \sqrt{\mathrm{e}}} \mathrm{U}_{0} \mathrm{~d}_{0}
\end{aligned}
$$

The penetrated volume as a function can be found by substituting Eq. (4.40) into Eq. (4.22):

$$
\mathrm{V}(\mathrm{t})=72 \pi \theta^{2} \frac{\mathrm{d}_{0}^{3}}{\gamma^{3 / 2}}\left(1-\left(1-\frac{\gamma \sqrt{\mathrm{e}} \mathrm{t} \mathrm{U}_{0}}{6 \mathrm{~d}_{0}}\right)^{2}\right)^{3 / 2}
$$

The volume of gas contained in the swept volume is

$$
\mathrm{V}_{\mathrm{gas}}=\alpha \mathrm{pV}
$$

So from Eq. (4.42) we obtain

$$
\mathrm{V}_{\text {gas }}(\mathrm{t})=72 \pi \theta^{2} \alpha \mathrm{p} \frac{\mathrm{d}_{0}^{3}}{\gamma^{3 / 2}}\left(1-\left(1-\frac{\gamma \sqrt{\mathrm{e}} \mathrm{t} \mathrm{U}_{0}}{6 \mathrm{~d}_{0}}\right)^{2}\right)^{3 / 2}
$$

The maximum gas release according to the model is 


$$
\mathrm{V}_{\max }=72 \pi \theta^{2} \alpha \mathrm{p} \frac{\mathrm{d}_{0}^{3}}{\gamma^{3 / 2}}=72 \pi \theta^{2} \alpha \mathrm{p}\left(\frac{\rho}{2 \tau}\right)^{3 / 2}\left(\mathrm{U}_{0} \mathrm{~d}_{0}\right)^{3}
$$

From Eq. (4.45) we can write Eq. (4.44) as

$$
\frac{\mathrm{V}_{\mathrm{gas}}}{\mathrm{V}_{\max }}=\left(1-\left(1-\frac{\mathrm{t}}{\mathrm{t}_{\max }}\right)^{2}\right)^{3 / 2}
$$

For short times, the nonlinear term in Eq. (4.46) can be approximated to obtain

$$
\frac{\mathrm{V}_{\mathrm{gas}}}{\mathrm{V}_{\max }} \approx\left(\frac{2 \mathrm{t}}{\mathrm{t}_{\max }}\right)^{3 / 2} \quad \frac{\mathrm{t}}{\mathrm{t}_{\max }}<<1
$$

In terms of the physical parameters, Eq. (4.47) can be written

$$
\mathrm{V}_{\text {gas }}(\mathrm{t}) \approx \frac{72}{\sqrt{27}} \pi \theta^{2} \alpha \mathrm{p}(\mathrm{e})^{3 / 4}\left(\mathrm{U}_{0} \mathrm{~d}_{0} \mathrm{t}\right)^{3 / 2} \quad \mathrm{t}<<\frac{3 \rho}{\tau \sqrt{\mathrm{e}}} \mathrm{U}_{0} \mathrm{~d}_{0}
$$

Eq. (4.47) and (4.48) are referred to as the linearized model, which applies only during short times, as quantified in the equations. Figure 4.17 plots Eq. (4.46) and (4.47) together.

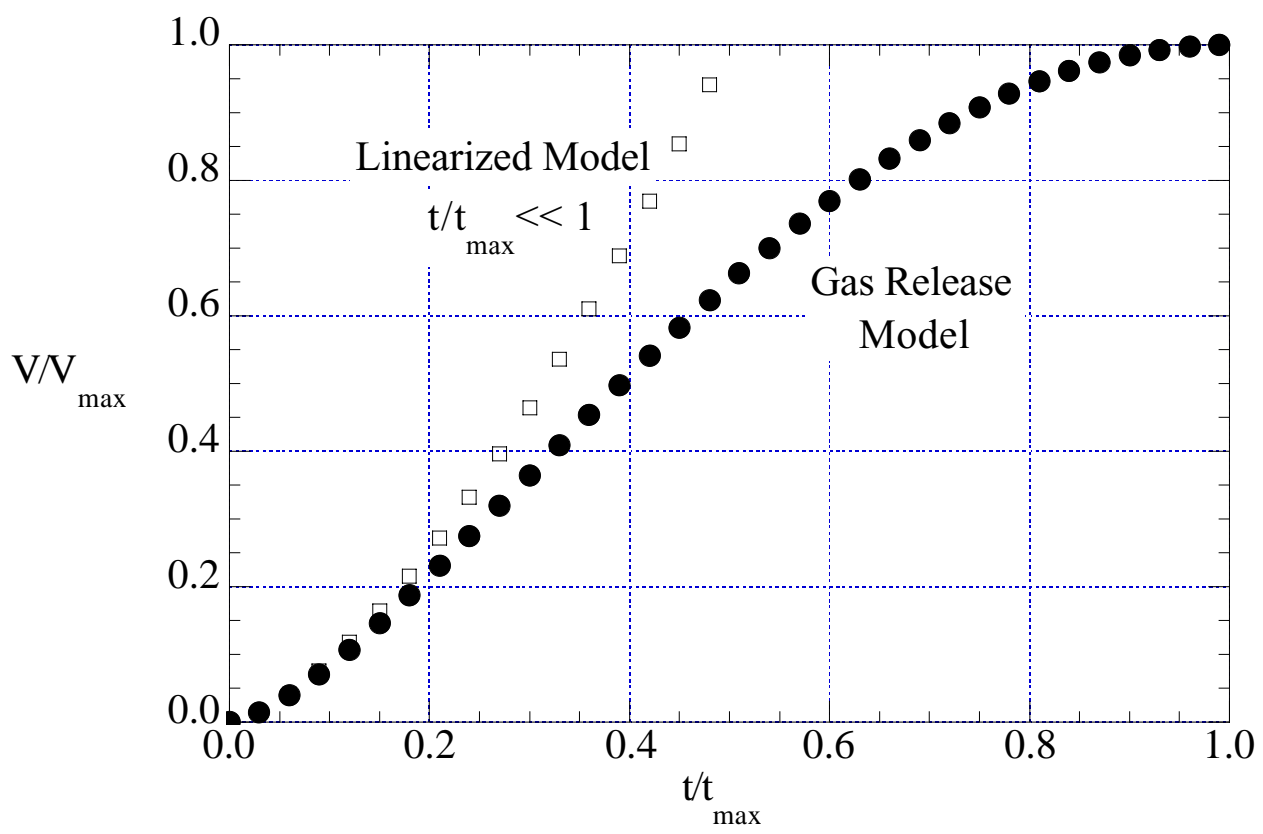

Figure 4.17. Gas Release Model Results for Eq. (4.46) and (4.47) 
We can make several observations from these modeling results:

- The gas release volume depends on all the parameters initially expected, ${ }^{(a)}$ which are shown in Eq. (4.1).

- Unlike the correlation attempts made in Section 4.2, the time dependence is not simple because it is both nonlinear and dependent on a number of parameters.

- The cubic dependence on $\mathrm{U}_{0} \mathrm{~d}_{0}$ in Eq. (4.45) is unlike jet volume flow, momentum, or power.

- For short times, the penetrated volume (and associated gas release) does not depend on shear strength or density (Equation (4.48)).

\subsubsection{Gas Release Model}

We now apply the model derived in the previous section. We begin by using gas release data from SY-101 mixer pump operation to demonstrate the functional form of the model and adjust the free constants as appropriate. The model is then applied to conditions in Tank AN-105.

\subsubsection{Results for Tank SY-101}

We now wish to evaluate Eq. (4.44) for the conditions corresponding to the various SY-101 pump runs discussed in Section 4.2. Table 4.3 shows the pertinent parameters for the tank waste and pump runs. Also shown are the parameter $\gamma$ and $\sqrt{e t}_{\text {max }}$ (e is left explicit for now because it will need to be evaluated from the data). For the pump runs where the discharge was ramped up, gas release was computed for each step; then the total was computed as the sum of the individual step contributions. Figure 4.18 shows a comparison of the gas release computed with Eq. (4.44)

Table 4.3. Model Input Parameters for Tank SY-101 Mixer Pump Tests

\begin{tabular}{||l|c|c|c|c||}
\hline \multicolumn{1}{||}{ Date } & $\mathbf{U}_{\mathbf{0}}(\mathbf{m} / \mathbf{s})$ & $\mathbf{g}$ & $\begin{array}{c}\text { Duration } \\
(\mathbf{m i n})\end{array}$ & $\begin{array}{c}\mathbf{e}^{\mathbf{1 / 2}} \mathbf{t}_{\mathbf{m a x}} \\
(\mathbf{s})\end{array}$ \\
\hline $7 / 26 / 93$ & 21.1 & 0.00056 & 9.3 & 33.4 \\
\hline $10 / 22 / 93$ & 11.0 & 0.00256 & 19.7 & 15.7 \\
\hline $11 / 10 / 93$ & 7.9 & 0.00424 & 20 & 12.2 \\
\hline $11 / 5 / 93$ & 7.9 & 0.00401 & 19.7 & 12.5 \\
$11 / 11 / 93$ & 11.0 & 0.00207 & 19.7 & 17.4 \\
$11 / 12 / 93$ & 15.3 & 0.00107 & 19.7 & 24.2 \\
$11 / 13 / 93$ & 19.6 & 0.00065 & 19.7 & 31.0 \\
\hline $11 / 21 / 93$ & 15.9 & 0.00100 & 60 & 25.0 \\
\hline For all runs: $\mathrm{d}_{0}=0.066 \mathrm{~m}, \rho=1500 \mathrm{~kg} / \mathrm{m}^{3}, \tau=200 \mathrm{pa}, \alpha=.08, \mathrm{p}=2$. \\
\hline
\end{tabular}

(a) Exceptions are density difference and viscosity. Buoyancy effects associated with the density difference have not been treated to simplify the analysis. The viscosity is treated implicitly because turbulent jet theory applies to flows with large Reynolds number and hence the viscosity is not important. In actuality, the flow near the penetrating front may be slow enough that viscous effects are important. 


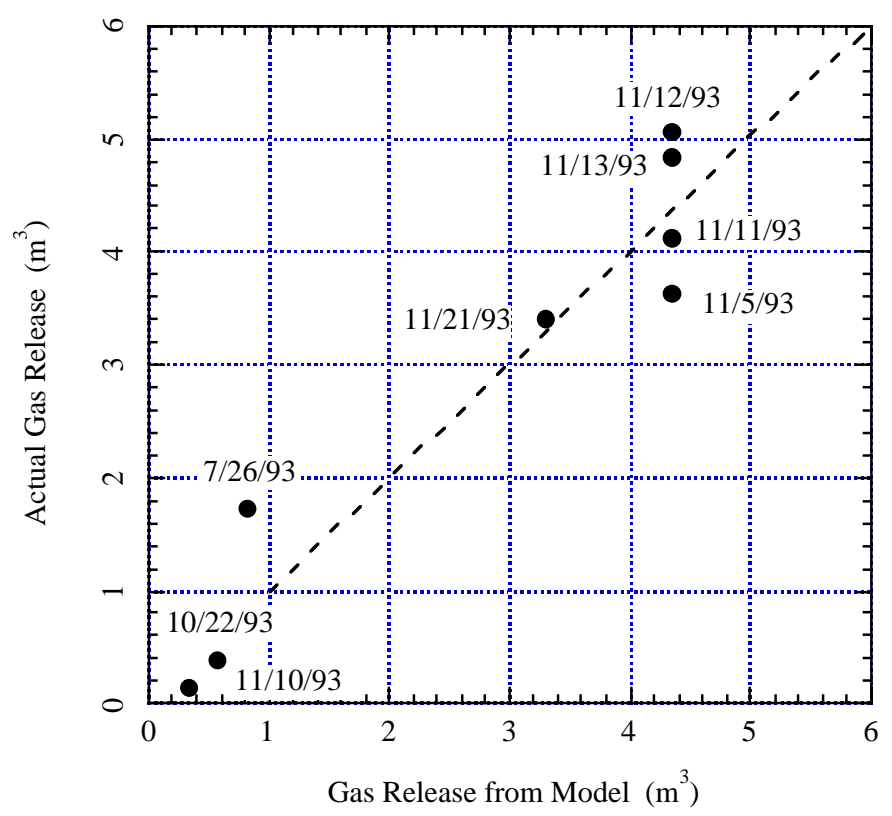

Figure 4.18. Comparison of Predicted Gas Release from Model (Eq. 4.44) and Observed Releases in SY-101 (data from Table 4.4; $\theta=4.7$ degrees and $\mathrm{e}=0.00005$ )

and the actual gas releases from the mixer pump runs. The jet spread angle $\theta$ was set to 4.7 degrees, and the efficiency, e, was set to 0.00005 . The reduced jet spread angle is consistent with the expectation that the finite strength of the sediment layer reduces entrainment. The efficiency, however, is quite low. From Eq. (4.25) we expect an efficiency of 0.036 if the average jet power is completely converted to yielding the sediment layer. The value of 0.00005 is only $0.2 \%$ of this value, suggesting the process is very inefficient. A better explanation, perhaps, is that much of the useful power of the jet is not being applied to the process of penetration. This is consistent with the observation from SY-101 thermocouple data, which suggests the jet becomes quite buoyant and finds a "leak" path to the top of the sediment layer rather quickly.

\subsubsection{Results for Tank AN-105}

Using the values in Table 4.4, Eq. (4.44) and (4.48) are evaluated ${ }^{(a)}$ and become

$$
\mathrm{V}_{\text {gas }}=16.8\left(1-\left(1-\frac{\mathrm{t}}{125}\right)^{2}\right)^{3 / 2}
$$

(a) The gas release model was derived for a single jet. Hence, when applying to mixer pumps systems, the results are multiplied by 2 . 
Table 4.4. Model Parameter Values for Tank AN-105

\begin{tabular}{||l|l|}
\hline Parameter & Value \\
\hline Nozzle discharge velocity $\mathrm{U}_{0}(\mathrm{~m} / \mathrm{s})$ & $11.6 \mathrm{~m} / \mathrm{s}$ \\
\hline Nozzle diameter $\mathrm{d}_{0}(\mathrm{~m})$ & 0.152 \\
\hline Nonconvective layer density $\rho\left(\mathrm{kg} / \mathrm{m}^{3}\right)$ & 1500 \\
\hline Yield strength $\tau(\mathrm{Pa})$ & 150 \\
\hline Average void fraction $\alpha$ & 0.06 \\
\hline Nonconvective layer average pressure ratio $\mathrm{p}$ & 2 \\
\hline Jet spread angle $\theta$ (degrees) & 4.7 \\
\hline Penetration Efficiency e & 0.00005 \\
\hline
\end{tabular}

$$
\mathrm{V}_{\text {gas }} \approx 0.034(\mathrm{t})^{3 / 2} \quad \mathrm{t}<<125 \min
$$

where $t$ has been changed to have units of minutes and gas release volume remains in cubic meters. Eq. (4.49) and (4.50) are plotted in Figure 4.19.

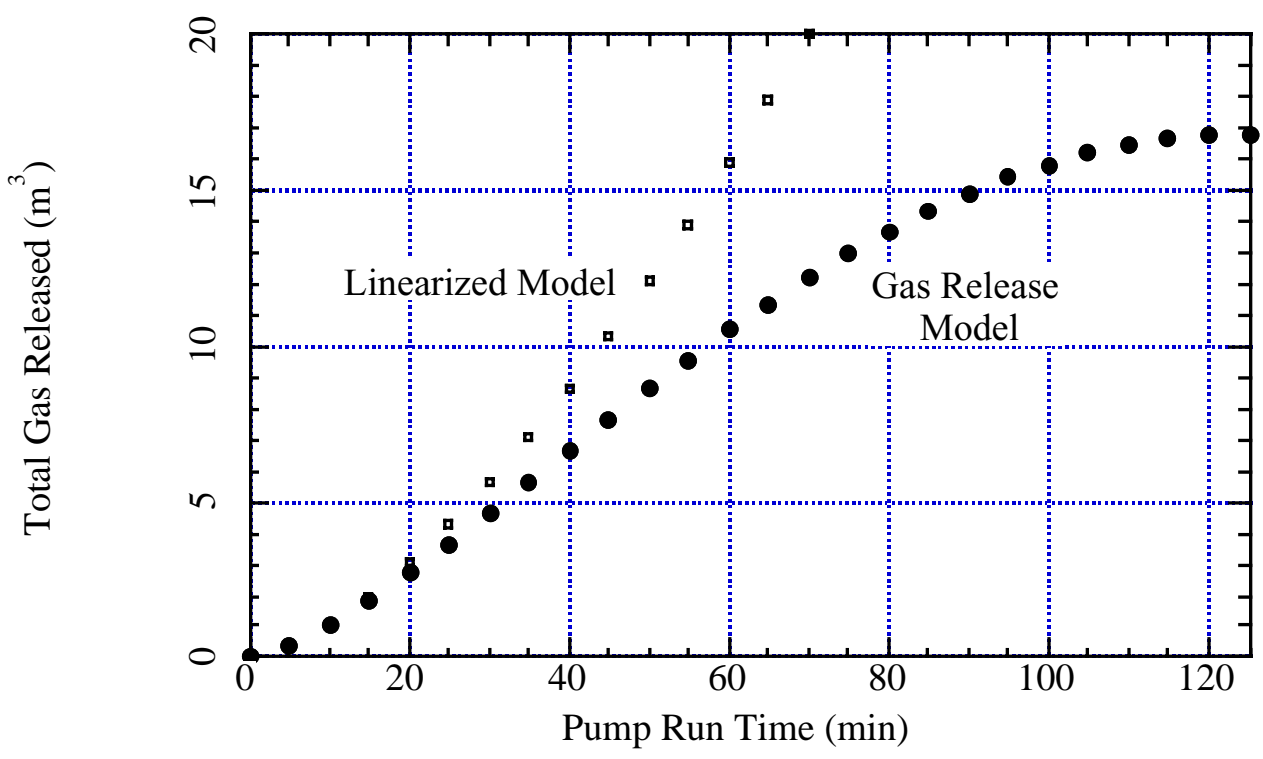

Figure 4.19. Predicted Gas Release Volume from Operation of a Mixer Pump in Tank AN-105

\subsubsection{Recommendations for Further Modeling and Validation}

This section presents a discussion of the limitations and uncertainties associated with the gas release model and presents suggestions for further modeling and validation. 


\subsubsection{Model Limitations and Uncertainties}

Mixer pump induced gas release in Hanford waste tanks is complex. The models presented should be thought of as a first-order attempt to capture some of the important features of the process. There are a number of model limitations and uncertainties that should be strengthened in order to have confidence in actual mixer-pump induced gas release behavior. The following is a brief list and discussion of various issues:

- The model does not account for buoyant effects, yet buoyancy was clearly an important feature in the data set from Tank SY-101, which was used for model calibration.

- The small value of the efficiency parameter e is consistent with the observed behavior in SY-101-that the jet quickly penetrated the top of the sediment layer due to buoyancy. Larger values of e are likely if buoyancy effects are minimized (as will be the case for Tank AN-105). This would likely cause the gas release to occur more quickly.

- Gas release from the model "turns off" after the maximum jet penetration is attained. Clearly, there would be additional gas release from whatever recirculating currents are established in the waste.

- The model does not consider collapse of sediment layer material into penetrated regions. Basic scaling suggests that collapse is very likely. Collapse could introduce additional gas retaining material into the path of the jet and thereby increase the overall amount of gas released.

- The model assumed uniform waste properties. Regarding yield stress, it has been observed from the ball rheometer data that yield stress tends to increase with increasing depth in the sediment layer. This type of yield stress gradient will likely cause the jet to turn upward. The net effect would likely be to decrease gas release.

- The model assumes nothing regarding the return path of the discharge flow. For SY-101, this is likely acceptable because the pump inlet was in the convective layer far from the nozzle and jet. For Tank AN-105, the lower inlet will likely result in a strong recirculating flow. The features of this flow are not clear, and the applicability of the current model to this situation is unclear.

\subsubsection{Recommendations}

To improve our ability to predict gas release in Tank AN-105 (and other tanks) there are several activities that would be beneficial:

- Extend the current model to account for buoyancy effects that may dominate the flow, depending on the location of the pump inlet.

- Develop a theoretical model for the recirculating jet that would likely be present. This would be based on a cavity-type flow for which there are some experimental data.

- Address the phenomenon of sediment layer collapse into the jet penetration region.

- Develop additional data for model validation. A great deal of information would be gleaned from lab-scale experiments with jets in simulated gas-retaining sediment layers. Because we are primarily after first-order behavior, many of the technical issues associated with developing representative simulants would not be of concern. 
- Perform computational fluid dynamics simulations (TEMPEST; Onishi and Trent 1998) with the goal of examining the penetration and collapse features. In this way, model development could be guided. Additionally, it is possible to develop a gas release model for the TEMPEST code. This feature would allow gas to be released as the pump is operated.

\subsection{Model for Direct and Induced Gas Releases}

Operation of a mixer pump in the sediment layer of a tank will serve to not only release gas from the disturbed region but to mix and suspend solids into the supernatant layer, which can induce BDGREs. Therefore, the effect of mixer pump operation on the headspace hydrogen concentration must include the possibility of induced BDGREs as well as the direct release of gas from the mixer pump operation.

\subsubsection{Modeling Mixer Pump Operations}

To investigate the flammable gas issues associated with mixer pump operation, that is, what effect does the amount of sediment material mobilized by a given pump operation or run have on the tank headspace hydrogen concentration, we have simply specified that a mixer pump operation will disturb a set amount of the sediment layer. The volume of sediment disturbed determines the volume of the retained gas released and the amount of material suspended into the supernatant liquid to potentially induce BDGREs. By varying the amount of sediment layer disturbed and observing the resulting headspace hydrogen concentration transient, we can determine bounds on mixer pump operation in terms of the maximum disturbed volume and the waiting time necessary between runs. The mixing model described in Section 4.1 can then be used to determine the pump speed and duration that will produce the given disturbance.

Modeling of the headspace hydrogen concentration during mixer pump operation was based on the following assumptions:

- All of the gas in the volume of sediment layer disturbed by the mixer pump is released.

- The gas is released into the headspace at a time-varying rate similar to that of a BDGRE.

- The mixing and suspension of the sediment is instantaneous.

To model BDGREs induced by suspension of sediment in the supernate, the mixer pump model was coupled with the decant model (with the decant rate set to zero) described in Section 3.1. This coupling also allowed us to use the initial conditions of gob count, size, and gas fraction distributions that were created for the decant analysis (see Section 3.2). The Monte Carlo simulation method was likewise used to produce probability distributions of the model results (see Section 3.2.5).

The pump operations or runs are modeled by specifying the volume of sediment layer material disturbed by each run. Mixer pump runs occur at set intervals in time until the entire sediment layer has been disturbed. During the mixer pump operations, BDGREs are allowed to occur in exactly the same fashion as presented in Section 3, except that now we have only the 
minimal depressurization due to gas release, so the effect of solids suspension during mixing is the dominant initiation mechanism. The total hydrogen concentration in the headspace is therefore a combination of mixer pump-driven releases and BDGREs.

The methodology for determining the characteristics of the sediment layer that will be affected by subsequent mixer pump operations (i.e., what gobs are affected) is presented in Section 4.2.2, and the changes to the decant model are discussed in detail in Section 4.2.3.

\subsubsection{Partitioning the Waste Disturbance Among Gobs}

Actual mixer pump operations in the tank will disturb different areas of the sediment layer as the pump is manipulated (e.g., changing orientation, speed, and duration). As was discussed in Section 3, the sediment layer consists of a collection of semi-independently evolving gobs of varying sizes with different degrees of gas retention and relative buoyancy. The gas release from a pump run therefore depends on which gobs or portions of gobs are affected by that run.

The decant model did not consider the actual geometric location of individual gobs, and, though nonphysical, the collection of circular gobs was assumed to occupy the entire circular tank area. The assumed shape, size, and gas fractions of these gobs are discussed in Section 3.2. The same assumptions are retained in the mixer pump model. To account for the gob or gobs disturbed by a pump run, the set of gobs is ordered randomly and disturbed sequentially.

A given fraction of the sediment layer is assumed to be disturbed by each mixer pump run. The first gob in the input set is disturbed first. If the gob's volume is less than the pump disturbed volume, that gob volume is set to zero and the second gob disturbed. If the second gob volume is less than the pump disturbed volume minus the volume of the first gob, then the third gob is affected, and so on. If the subsequent gob volume is greater than the remaining volume to be disturbed by the pump, the difference is subtracted from that gob, leaving a smaller gob with its original gas fraction to be disturbed on the next run or to experience a BDGRE.

This process illustrated in Figure 4.20. The input set of gobs is represented by the top row of circles. Their sizes represent the varying size and gas fraction of the input set. The total volume disturbed by the first mixer pump run is denoted by the vertically hashed ellipsoid. The "volume" of the mixer pump operation requires that the first gob is entirely disturbed, while just a portion of the second gob is disturbed. The second mixer pump run, which occurs a set interval after the first run, is represented by the horizontally slashed ellipsoid, and it disturbs the remainder of the second gob, all of the third gob, and a portion of the fourth gob. This process continues until all of the gobs (i.e., sediment layer volume) have been disturbed.

The disturbance of a gob results in all of the disturbed portions' gas being released at a timevarying rate and a fraction of its solids being suspended in the supernatant liquid for the duration of mixer pump operations. At any time during this process, BDGREs may be induced, as described in Sections 2 and 3. If, for example, the third gob in Figure 4.20 had an induced BDGRE between the first and second mixer pump operations, the gob's bulk and gas volumes would have been reduced, as specified in Section 3. The second mixer pump run would then have disturbed this altered gob, and more of the fourth gob would have been disturbed. 


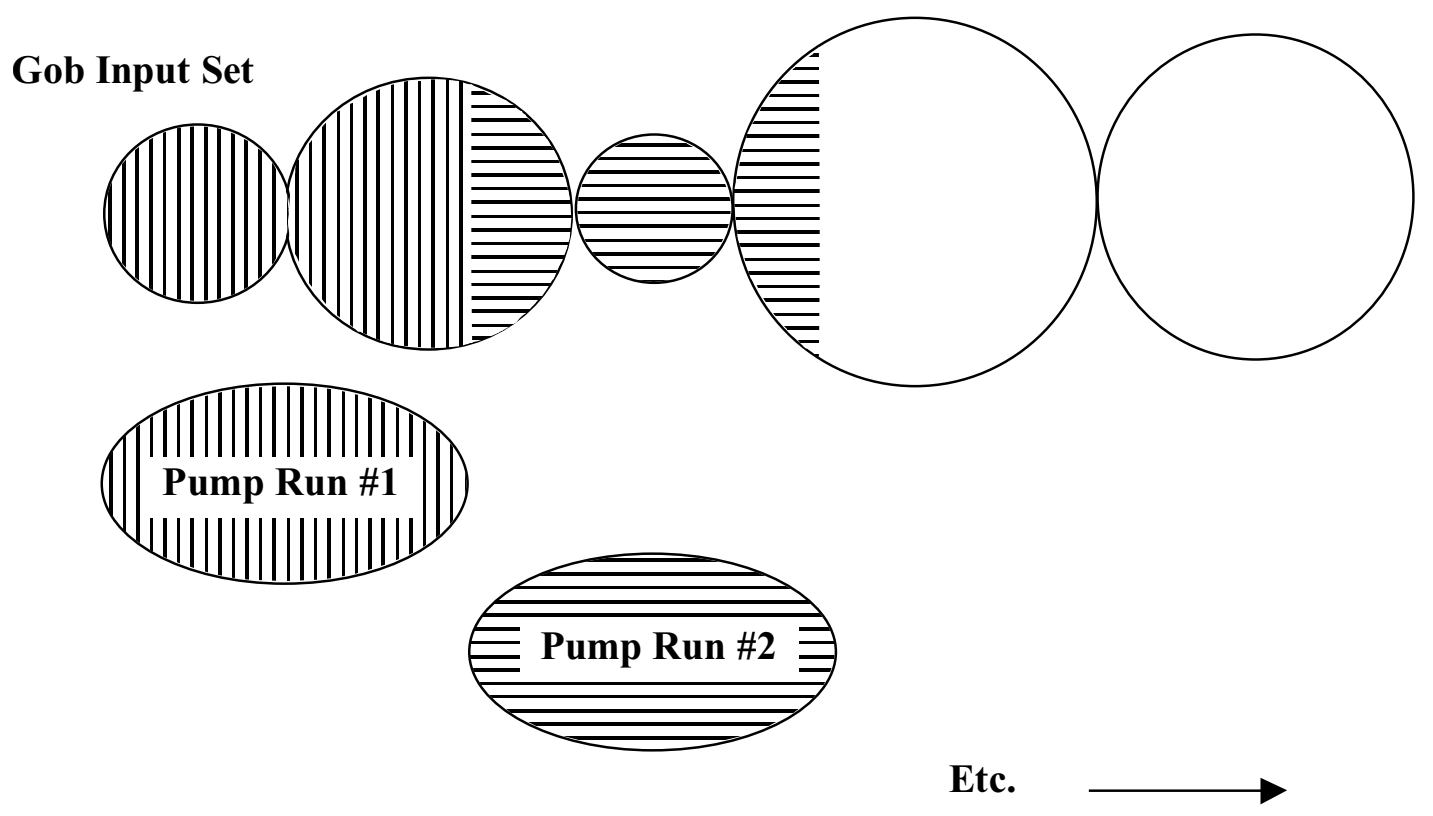

Figure 4.20. Schematic of Mixer Pump Operation and Effect on Gob Distribution

The bulk of the changes to the decant model are therefore bookkeeping steps to account for whether a portion or all of a gob has been disturbed by the mixer pump and whether those gobs or portions of gobs may experience a BDGRE. Those gobs that do experience BDGREs must then be "tracked" after their release for future mixer pump disturbance.

\subsubsection{Modification of Decant Model}

As discussed, the mixer pump model was coupled into the decant model presented in Section 3.1. All modeling remains the same unless otherwise noted in this section. The bulk sediment layer volume disturbed by a mixer pump run is given by

$$
\mathrm{V}_{\mathrm{Pm}}=\Psi \mathrm{h}_{\mathrm{S}} \mathrm{A}_{\mathrm{T}}
$$

where the subscript $m$ denotes which mixer pump operation, $\Psi$, is the fraction of the sediment layer volume disturbed by a single pump operation; $h_{S}$ is the sediment layer depth; and $A_{T}$ is the tank area. This disturbed volume is composed of a gob or gobs, as discussed above. The initial bulk gob volume is computed from

$$
\mathrm{V}_{\mathrm{gb} 0, \mathrm{n}}=\frac{\pi}{4} \mathrm{D}_{\mathrm{n}}^{2} \mathrm{~h}_{\mathrm{S}}
$$

where the subscript $\mathrm{n}$ denotes the gob and $\mathrm{D}$ is the gob diameter (see Section 3.2.2). Mixer pump operations or a BDGRE can change the gob volume by

$$
\mathrm{V}_{\mathrm{gbi}, \mathrm{n}}=\mathrm{V}_{\mathrm{gbi}-1, \mathrm{n}}-\mathrm{V}_{\mathrm{Pm}, \mathrm{n}}-\frac{\mathrm{V}_{\mathrm{re} \ln \mathrm{i}-1(\mathrm{BDGRE})}}{\mathrm{P}_{\mathrm{S}}}-\mathrm{V}_{\mathrm{gobSi}-1, \mathrm{n}(\mathrm{BDGRE})}
$$


where the subscript $\mathrm{i}$ denotes the time step. The subscript (BDGRE) implies that a BDGRE occurred at the previous time step in that gob. Therefore, $\frac{V_{\text {re ln } i-1(B D G R E)}}{P_{S}}$ is the total in situ gas volume released from the gob when the gob was released at the preceding time step (term is zero otherwise) where $P_{S}$ is the average hydrostatic pressure in the sediment layer (see Eq. 3.1 and 3.23). Likewise, $\mathrm{V}_{\text {gobSi-1,n(BDGRE) }}$ is the total volume of nongaseous gob material suspended into the supernate when the gob was released at the preceding time step (term is zero otherwise; see Eq. 3.27-3.29).

The total gas released from a gob in the disturbed region is

$$
\mathrm{V}_{\text {PGi,n,m }}=\mathrm{V}_{\text {gasAi,n }}\left(1-\frac{\mathrm{V}_{\mathrm{gbni}}}{\mathrm{V}_{\mathrm{gbni}-1}}\right)
$$

where $\mathrm{V}_{\text {gasAi,n }}$ is the average volume of gas in the gob computed by

$$
\mathrm{V}_{\mathrm{gasAi,n}}=\alpha_{\mathrm{Ai}, \mathrm{n}} \mathrm{V}_{\mathrm{gbi}, \mathrm{n}}-\mathrm{V}_{\mathrm{PGi}, \mathrm{n}}-\frac{\mathrm{V}_{\mathrm{re} \ln \mathrm{i}-1(\mathrm{BDGRE})}}{\mathrm{P}_{\mathrm{Si}-1}}
$$

where $\alpha_{\mathrm{Ai}, \mathrm{n}}$ is the average gas fraction in a gob (Eq. 3.8). If the gob has already experienced a BDGRE, the average volume of gas in the gob is given by

$$
\mathrm{V}_{\text {gasAi,n }}=\mathrm{V}_{\text {gasAi-1,n }}-\mathrm{V}_{\mathrm{PGi}, \mathrm{n}}
$$

The total pump gas release rate at atmospheric conditions is

$$
\mathrm{V}_{\mathrm{PGi}}=\frac{1}{\Delta \mathrm{t}} \sum_{\mathrm{n}=1}^{\text {disturbed_gobs }} \mathrm{V}_{\mathrm{PGi}, \mathrm{n}} \mathrm{P}_{\mathrm{Si}}
$$

This gas volume is released into the tank headspace at a time varying rate computed equivalently to Eq. (3.26) as

$$
\mathrm{Q}_{\mathrm{PGi}}=\frac{\mathrm{V}_{\mathrm{PGi}}}{\Delta \mathrm{t}}\left\lfloor\left(\frac{\mathrm{t}_{\mathrm{i}}-\mathrm{t}_{0 \mathrm{n}}}{\tau_{\mathrm{P}}}+1\right)^{\frac{\mathrm{t}_{0 \mathrm{n}}-\mathrm{t}_{\mathrm{i}}}{\tau_{\mathrm{P}}}}-\left(\frac{\mathrm{t}_{\mathrm{i}+1}-\mathrm{t}_{0 \mathrm{n}}}{\tau_{\mathrm{P}}}+1\right) \mathrm{e}^{\frac{\mathrm{t}_{0 n}-\mathrm{t}_{\mathrm{i}+1}}{\tau_{\mathrm{P}}}}\right\rfloor
$$

where $\Delta \mathrm{t}$ is the time step, $\mathrm{t}_{0 \mathrm{n}}$ is the gas release start time, and $\tau_{\mathrm{P}}$ is the time to the peak release rate for mixer pump operations (see Section 4.3).

The headspace hydrogen concentration given by Eq. (3.39) is then 


$$
\mathrm{C}_{\mathrm{Hi}}=\frac{\mathrm{C}_{\mathrm{Hi}-1}-\frac{\left(\mathrm{Q}_{\mathrm{GASi}}+\mathrm{Q}_{\mathrm{PGi}}\right) \cdot \chi_{\mathrm{H}}}{\mathrm{V}_{\mathrm{HSi}}} \Delta \mathrm{t}}{1+\frac{\left(\mathrm{Q}_{\mathrm{VIN}}+\mathrm{Q}_{\mathrm{GASi}}+\mathrm{Q}_{\mathrm{PGi}}\right)}{\mathrm{V}_{\mathrm{HSi}}} \Delta \mathrm{t}}
$$

where $\chi_{\mathrm{H}}$ is the volume fraction of hydrogen in the waste gas, $\mathrm{Q}_{\mathrm{GAS}}$ is the release rate of gas from BDGREs (see Eq. 3.26), $\mathrm{Q}_{\mathrm{VIN}}$ is the headspace vent rate, and $\mathrm{V}_{\mathrm{HS}}$ is the headspace volume (Eq. 3.32). The supernatant liquid level in Eq. (3.32) is now given by

$$
\mathrm{h}_{\mathrm{Li}}=\mathrm{h}_{\mathrm{Li}-1}-\frac{\Delta \mathrm{t}}{\mathrm{A}_{\mathrm{T}}}\left(\frac{\mathrm{Q}_{\mathrm{GASi}-1}}{\mathrm{P}_{\mathrm{Si}-1}}+\frac{\mathrm{Q}_{\mathrm{PGi}}}{\mathrm{P}_{\mathrm{Si}}}\right)
$$

Solids from disturbed gobs mix into the supernate layer, and a fraction of these solids is assumed to remain suspended, increasing the liquid density. The nongaseous sediment layer volume disturbed by pump operation and suspended in the supernatant liquid is determined from

$$
\mathrm{V}_{\mathrm{PSi}, \mathrm{m}}=\mathrm{FP}\left(\mathrm{V}_{\mathrm{Pi}, \mathrm{m}}-\mathrm{V}_{\mathrm{PGi}, \mathrm{m}}\right)
$$

where FP is the fraction of the solids that remain suspended (see Section 4.3).

The liquid density $\left(\rho_{\mathrm{L}}\right)$ at time i must now account for the suspension of solids from mixer pump operation and BDGREs, and is given by

$$
\rho_{\mathrm{Li}}=\frac{\left(\mathrm{h}_{\mathrm{Li}} \mathrm{A}_{\mathrm{T}}+\sum_{\mathrm{t}=1}^{\mathrm{i}-1} \mathrm{~V}_{\mathrm{gobSt}}+\sum_{\mathrm{t}=1}^{\mathrm{i}-1} \mathrm{~V}_{\mathrm{PSt}}\right) \rho_{\mathrm{Li}-1}+\left(\mathrm{V}_{\mathrm{gobSi}}+\mathrm{V}_{\mathrm{PSi}}\right) \rho_{\mathrm{S}}}{\mathrm{h}_{\mathrm{Li}} \mathrm{A}_{\mathrm{T}}+\sum_{\mathrm{t}=1}^{\mathrm{i}-1} \mathrm{~V}_{\mathrm{gobSt}}+\sum_{\mathrm{t}=1}^{\mathrm{i}-1} \mathrm{~V}_{\mathrm{PSt}}+\mathrm{V}_{\mathrm{gobSi}}+\mathrm{V}_{\mathrm{PSi}}}
$$

\subsection{Parameter Definitions and Constraints}

With the exception of the time to the peak gas release rate for mixer pump operations, $\tau_{\mathrm{p}}$, and the fraction of solids mobilized by the pump that remain suspended, FP, the input distributions were exactly equivalent to those used in the decant model as presented in Section 3.2 and shown in Appendix C.

Gas releases due to mixer pump operations were evaluated on the initial operations of the SY-101 mixer pump (discussed in Section 4.1.2). The time to the peak gas release rate was determined from hydrogen concentration histories (Section 4.1.2 and Table 4.2) via the methods in Section 2.3. As with spontaneous BDGREs, no definitive value for $\tau_{\mathrm{P}}$ was identifiable. Thus, for each simulation a $\tau_{\mathrm{P}}$ was assigned from the distribution created from the SY-101 analysis (Figure 4.21). 
As discussed, solids suspended into the liquid layer can induce BDGREs. The spontaneous releases observed in SY-101 during initial mixer pump operations may have been caused by this phenomenon. A distribution for the fraction of the solids mobilized by the pump that remain suspended was assigned based on the mixer pump performance in SY-101. Prior to disturbance by the mixer pump, the sediment layer in SY-101 was approximately $5.8 \mathrm{~m}$ deep. After the tank was fully mixed, the unsuspended settled solids depth was estimated to be approximately 1.25 to $2 \mathrm{~m}$ (50 to $80 \mathrm{in}$.). These results indicate that approximately 60 to $80 \%$ of the solids from the sediment layer were suspended. These results are corroborated by the solid particle sizes measured in SY-101 (Reynolds 1993), which indicate that about 75\% of the solids would have settling velocities such that they would remain suspended for significant periods. Because a significant fraction of larger particles would settle out quickly, long-term suspension of $100 \%$ of the solids was assigned a zero probability. With no data defining a lower end, a symmetric distribution was chosen, as shown in Figure 4.22.

Values for the time to the peak gas release rate for mixer pump operations and the fraction of the solids mobilized by the pump that remain suspended were randomly selected from these distributions and formed additional inputs for the decant inputs sets. These are shown in Appendix C. As specified in Section 3.2.5, 10,000 runs were conducted in a Monte Carlo simulation for each retrieval operation, so the outputs represent all possible physical scenarios given the conditions constraining the inputs.

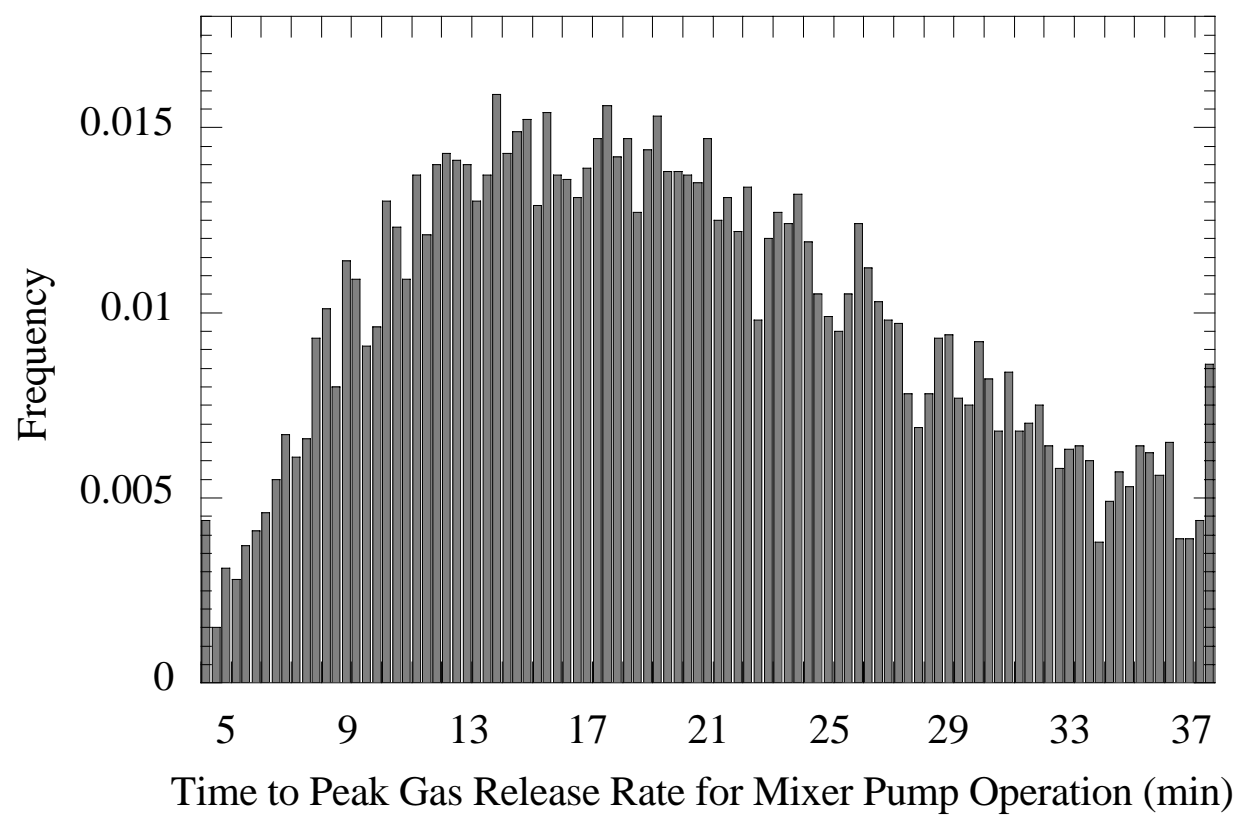

Figure 4.21. Distribution for Time to Peak Gas Release Rate for Mixer Pump Operations 


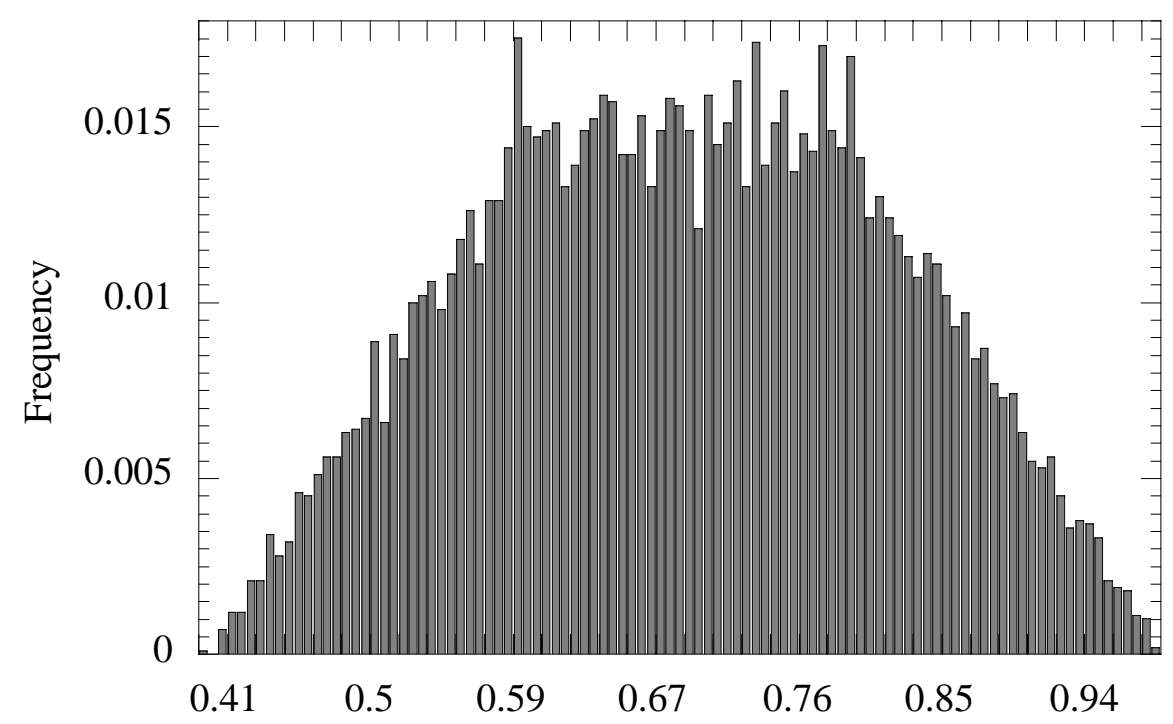

Fraction of Solids Mobilized by the Pump that Remain Suspended

Figure 4.22. Distribution for Fraction of Solids, Mobilized by Pump, that Remain Suspended

\subsection{Analysis of Mixing-Induced Gas Releases in AN-105}

As with the supernate decant simulations, Tank AN-105 was selected for special consideration for mixer pump-induced gas releases because 1) it has experienced BDGREs with the highest headspace hydrogen concentration, 2) it is one of the first tanks scheduled to be retrieved for vitrification, and 3) it is representative of the other tanks. The results of mixing analyses for the other three tanks (AN-103, AN-104, and AW-101) are presented in Section 4.5.

The mixing model developed in Section 4.1 assumes the flow from the pump nozzles behaves as a turbulent jet impinging on and slowly penetrating a wall of weak solid material. The net hydraulic power of the jet deposited on the waste at the penetration zone is set equal to the power required to yield the solid matrix. Jet theory applied to this energy balance shows that the maximum volume of waste disturbed is proportional to the quantity $\left(u_{0} d_{0}\right)^{3}$, where $u_{0}$ and $d_{0}$ are the initial jet velocity and nozzle diameter, respectively, while the time required to achieve this disturbance is proportional to $\mathrm{u}_{0} \mathrm{~d}_{0}$. The model was used to extrapolate gas release data from initial mixing tests in SY-101, at $150 \mathrm{hp}$, to degassing AN-105 with one of the 300-hp W-211 mixer pumps. The model predicts that running a single mixer pump in AN-105 at a fixed azimuth for about 26 minutes at $1200 \mathrm{rpm}$ (maximum speed) will theoretically mobilize 5\% of the sediment volume. A 76-minute run at the same speed mobilizes about $20 \%$ of the sediment. Combinations of pump speed and run time to achieve disturbances of 5,10, and $20 \%$ of the sediment are given in Table 4.5. Maximum theoretical disturbance at the given speed is also listed.

Cases were run (mixer pump operations were conducted at the set schedule until the entire sediment layer was disturbed) to investigate the sensitivity of the peak hydrogen concentration during mixing to the pump operation as follows: 
1. Base Case: Mixer pump runs are assumed to release gas from 5\% of the sediment layer per run. One run is made every 24 hours until the entire sediment layer has been mixed (20 runs).

2. Pump Speed Sensitivity: The base case was rerun with the fraction of sediment disturbed increased to $20 \%$. It was anticipated that larger disturbance would increase the peak hydrogen concentration in at least direct proportion. The $20 \%$ was selected as the maximum practical fraction of waste disturbed by one pump running at a fixed azimuth.

3. Pump Schedule Sensitivity: The base case was rerun with one pump run every 8 hours.

The input conditions are described in Sections 3.2 and 4.3 and listed in Appendix C. A Monte Carlo simulation approach was used as outlined in Section 3.2.5, and results of each of these cases for AN-105 are presented in separate subsections below. The method of presentation of the results is the same as used in Sections 3.3 and 3.4. Results of the base case are shown in Section 4.4.1 and the pump speed sensitivity study in Section 4.4.2. The pump schedule sensitivity study results are presented in Section 4.4.3, and the overall conclusions of these studies are summarized in Section 4.4.4. The results of the base case simulations and selected other cases for the other three tanks are given in Section 4.5, and the mixing results are summarized in Section 4.6.

Table 4.5. Fraction of Sediment Disturbed for Given Mixer Pump Run Conditions

\begin{tabular}{|c|c|c|}
\hline Pump Speed & $\begin{array}{c}\text { Fraction of Sediment } \\
\text { Disturbed per Run }\end{array}$ & Run Time (min) \\
\hline \multirow{4}{*}{$1200 \mathrm{rpm}$} & 0.050 & 26 \\
\hline & 0.100 & 44 \\
\hline & 0.200 & 76 \\
\hline & $0.409(\max )$ & 199 \\
\hline \multirow{4}{*}{$1000 \mathrm{rpm}$} & 0.050 & 32 \\
\hline & 0.100 & 54 \\
\hline & 0.200 & 104 \\
\hline & $0.255(\max )$ & 170 \\
\hline \multirow{3}{*}{$900 \mathrm{rpm}$} & 0.050 & 35 \\
\hline & 0.100 & 62 \\
\hline & $0.193(\max )$ & 155 \\
\hline \multirow{3}{*}{$775 \mathrm{rpm}$} & 0.050 & 41 \\
\hline & 0.100 & 77 \\
\hline & $0.137(\max )$ & 138 \\
\hline \multirow{3}{*}{$700 \mathrm{rpm}$} & 0.050 & 48 \\
\hline & 0.100 & 110 \\
\hline & $0.102(\max )$ & 125 \\
\hline
\end{tabular}




\subsubsection{Base Case in AN-105}

The distribution of the peak hydrogen concentration for the base case is presented in Figure 4.23. This case had 5\% of the sediment layer disturbed every 24 hours. The median of the peak hydrogen concentration is 7,500 ppm and the $95 \%$ confidence limit is $15,100 \mathrm{ppm}$. The 95\% confidence limit exceeds $25 \%$ LFL (15,100 ppm corresponds to about 38\% LFL).

The majority of the simulations had induced BDGREs. The distribution for the fraction of the retained gas released by BDGREs during the simulations is shown in Figure 4.24. Similar quantities of the retained gas was released by BDGREs induced by the operation of the mixer pump as was released during the decant operations (Section 3.3). The median release fraction is 0.09 , and the $95 \%$ confidence level is 0.23 . The high frequency of 0.13 at zero gas release indicates that for 1,300 of the 10,000 simulations, no BDGREs were triggered.

The headspace hydrogen concentration for a simulation representing the $95 \%$ confidence level peak hydrogen concentration is plotted in Figure 4.25. The repeated pump runs can be seen in spikes every 24 hours. The varied concentration results over the pump runs from 80 to 380 hours are caused by the gas volume distribution in the gobs affected by the subsequent pump runs (see Section 4.2). The maximum concentration is caused by two induced BDGREs immediately following the fourth pump operation. A final BDGRE, induced at 384.5 hours, can be seen in the increased hydrogen concentration in the second to last pump run.

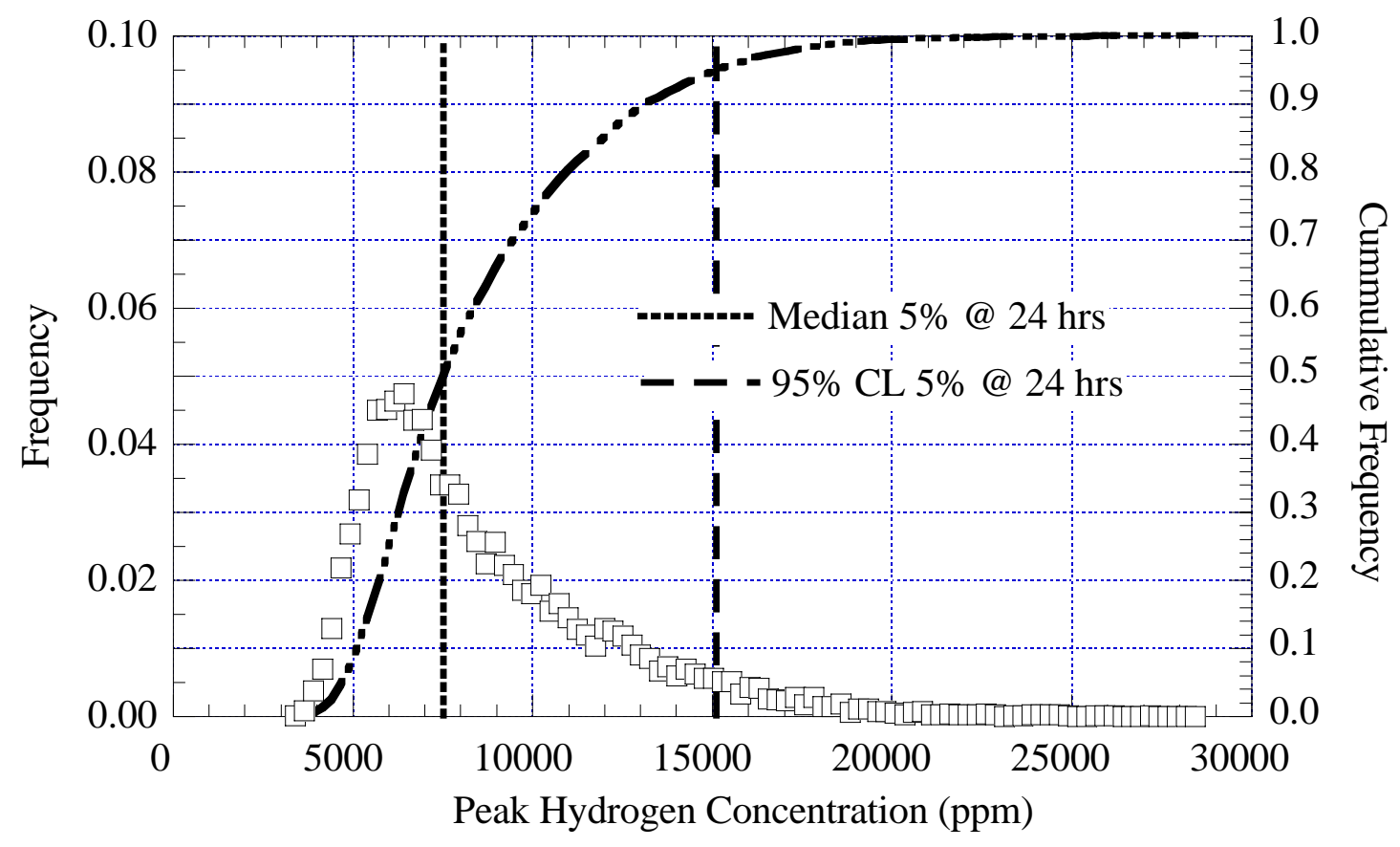

Figure 4.23. Peak Hydrogen Concentration: 5\% Disturbance Every 24 Hours in AN-105 


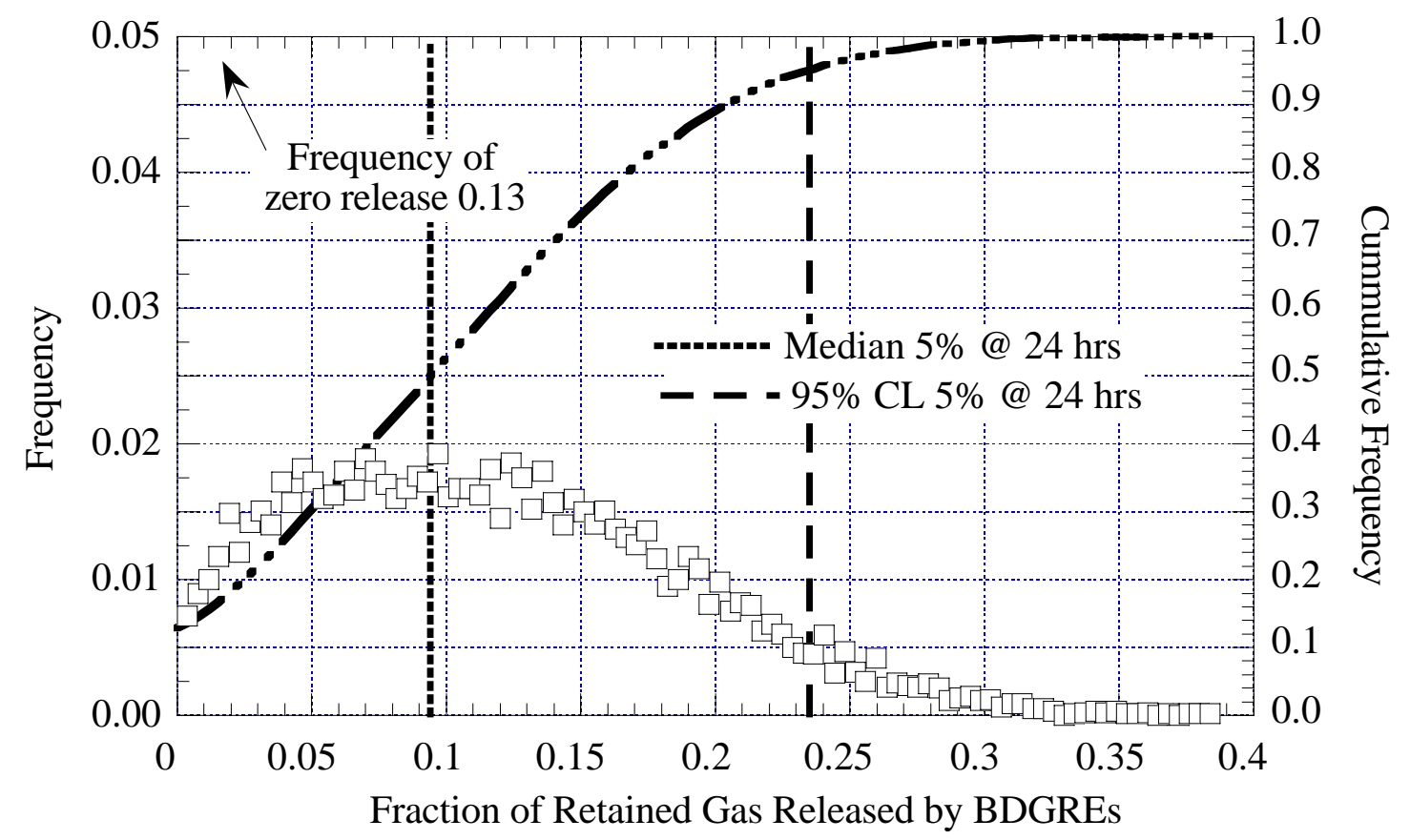

Figure 4.24. Gas Released by BDGREs: 5\% Disturbance Every 24 Hours in AN-105

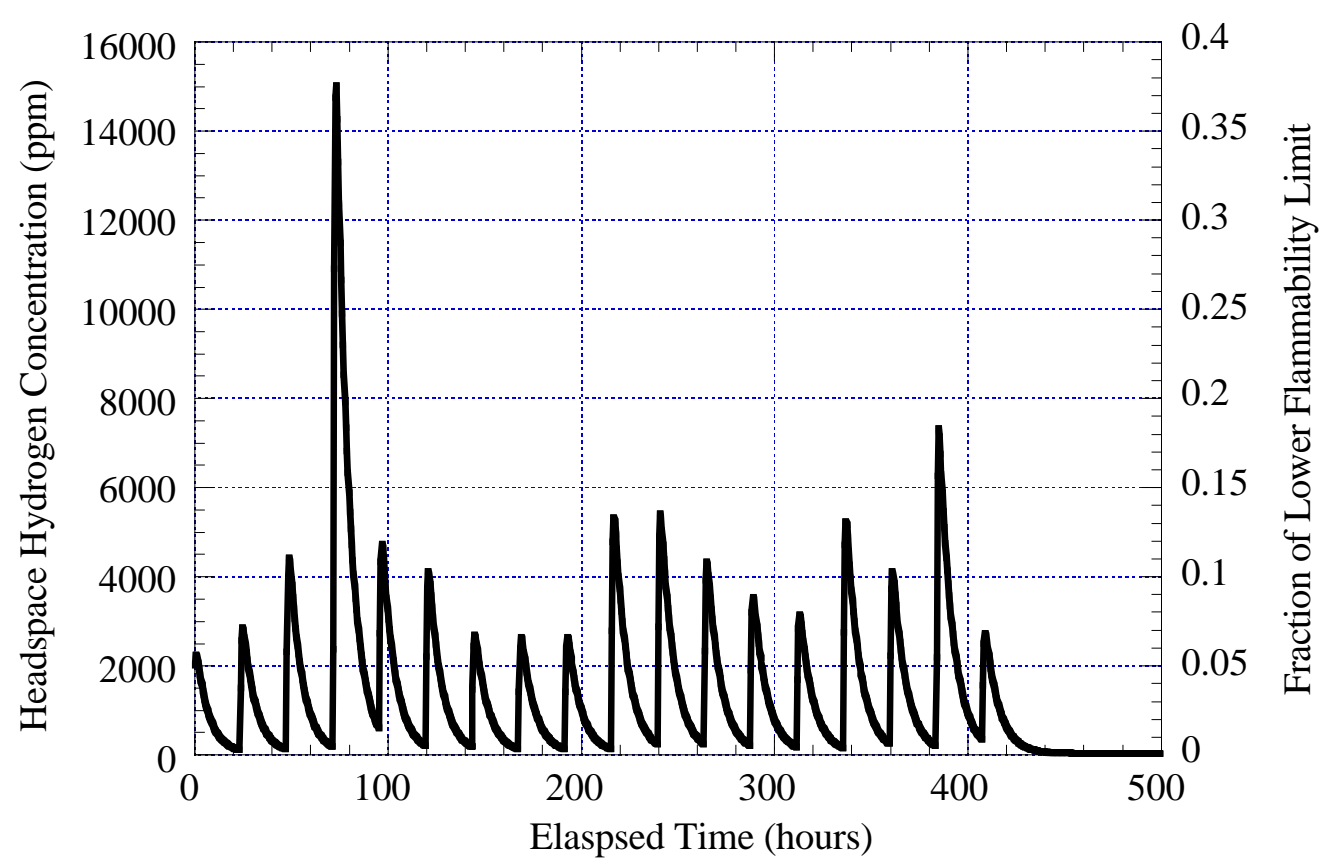

Figure 4.25. Example Simulation Hydrogen Concentration: 5\% Disturbance Every 24 Hours in AN-105 
The results show that, for $5 \%$ of the sediment layer disturbed by mixer pump operations every 24 hours, it is highly likely in the $95 \%$ confidence level that the hydrogen concentration will exceed $25 \%$ of the LFL. However, it is highly unlikely it will reach the LFL. The highest hydrogen concentrations during the mixer pump operation are caused by induced BDGREs adding to the gas release caused directly by the mixer pump. As with decant operations, the gas releases are more rapid than the ventilation rate (see Sections 3.2 and 4.3). Varying the ventilation rate for the mixer pump operations by a factor of two alters the predicted maximum hydrogen concentration at the $95 \%$ confidence level by approximately $15 \%$.

\subsubsection{Pump Speed Sensitivity}

The amount of sediment disturbed by the mixer pump operation is a function of the pump speed. The base case was rerun with the fraction of sediment disturbed increased to $20 \%$. It was anticipated that a larger disturbance would increase the peak hydrogen concentration.

Figure 4.26 shows the distribution of peak hydrogen concentrations for $20 \%$ of the sediment layer disturbed every 24 hours. The median and 95\% confidence level from the base case (5\% at 24 hours) are also shown. The median value increased from 7,500 ppm in the base case to $20,300 \mathrm{ppm}$ and the $95 \%$ confidence level from 15,100 to $28,400 \mathrm{ppm}$. In five of the 10,000 simulations, the peak hydrogen concentration exceeded the LFL. Clearly, increasing the amount of sediment layer material disturbed by a mixer pump run increases hydrogen concentration.

The fraction of the retained gas released by BDGREs during the simulations was slightly less than the base case (Figure 4.27). This results directly from the loss of gobs with the potential for BDGREs to the more vigorous effect of the mixer pump. A total of 1,785 simulations had no BDGREs, up from 1,300 in the base case.

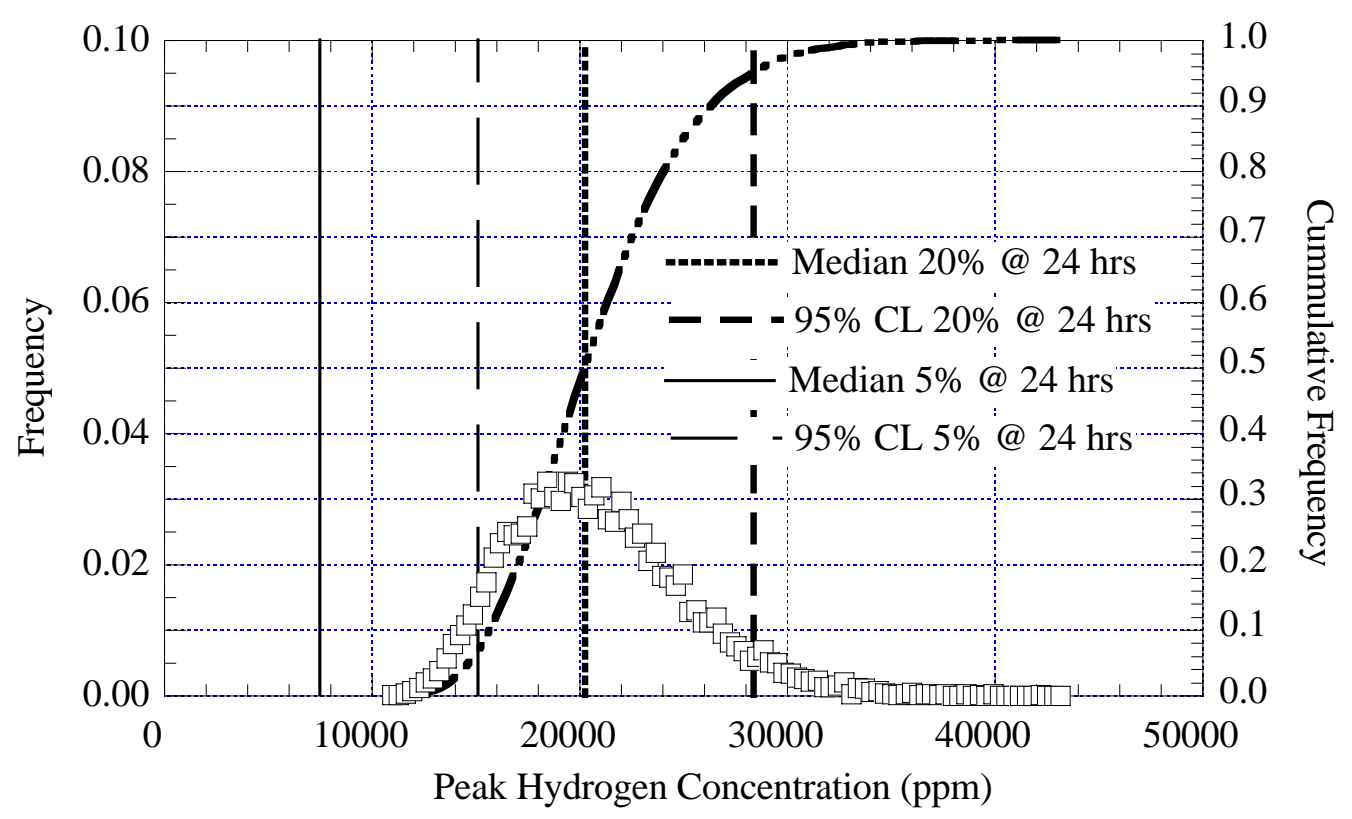

Figure 4.26. Peak Hydrogen Concentration: 20\% Disturbance Every 24 Hours in AN-105 


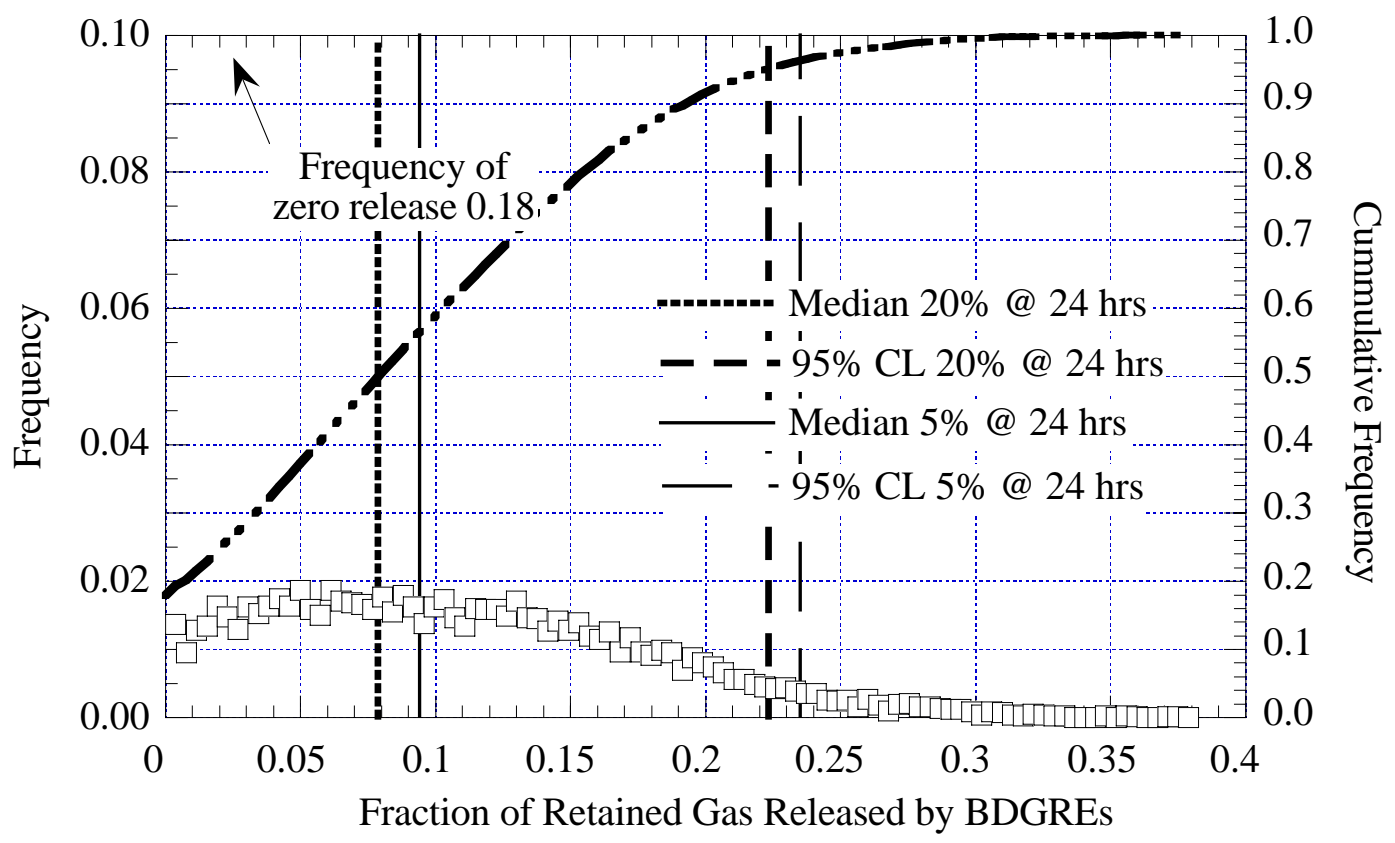

Figure 4.27. Gas Released by BDGREs: 20\% Disturbance Every 24 Hours in AN-105

The headspace hydrogen concentration for a simulation representing the 95\% confidence level of the peak hydrogen concentration (same simulation as base case, figure 4.25) is plotted in Figure 4.28. The spikes due to the pump runs are now significantly larger, as expected. The

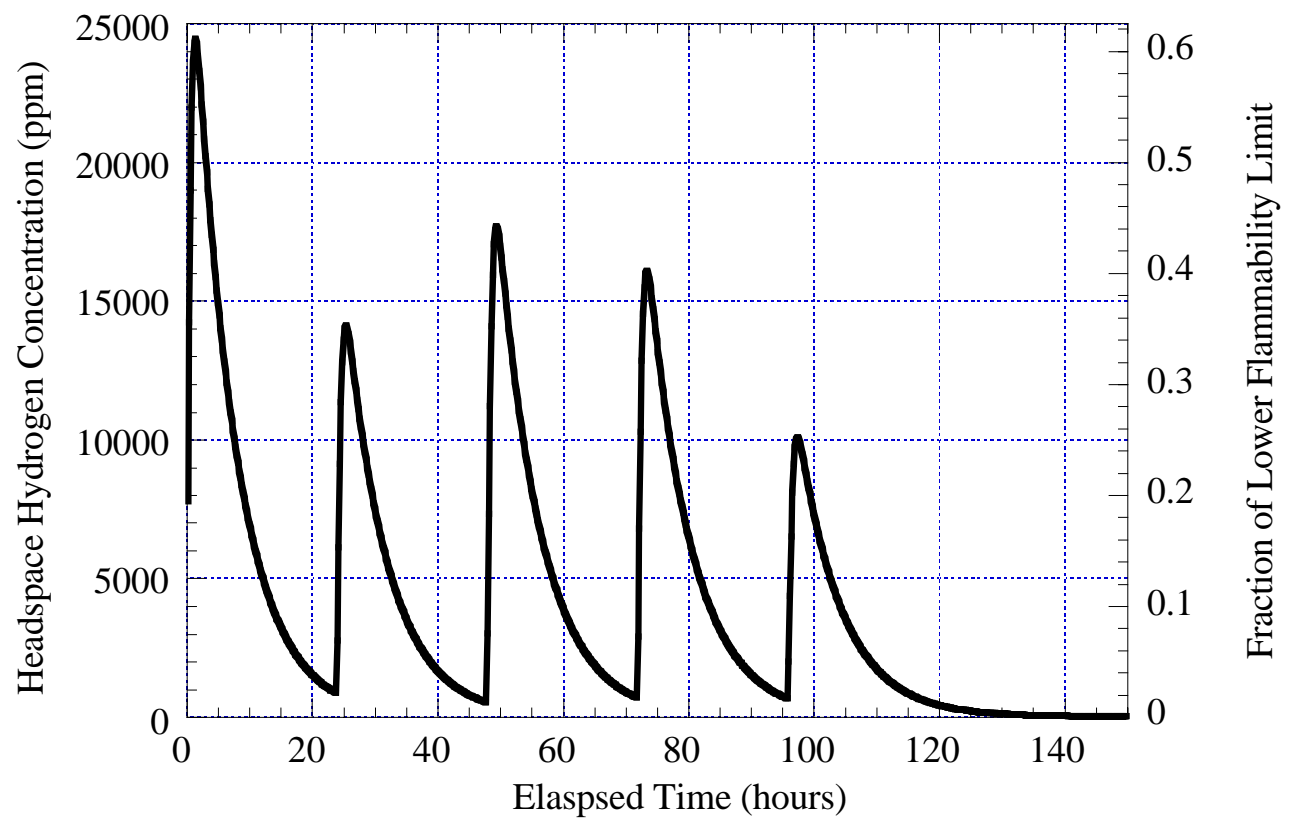

Figure 4.28. Example Simulation Hydrogen Concentration: 20\% Disturbance Every 24 Hours in AN-105 
same two gobs that are triggered after the fourth pump operation in the base case happen immediately after the first pump operation. The final BDGRE from the base case is not triggered because it is affected by the mixer prior to achieving buoyancy. Notice also that the total mixing time has reduced from approximately 410 hours to 100 hours.

\subsubsection{Pump Schedule Sensitivity}

The elevation of the hydrogen concentration by subsequent pump runs is postulated to be affected by altering the separation of the pump operations in time, allowing a longer or shorter time for the headspace to clear between runs. To investigate this, the base case was rerun with one pump run every 8 hours instead of every 24 hours.

The change in the distribution of the peak hydrogen concentration when the wait time between pump runs is decreased to 8 hours is presented in Figure 4.29. The median of the peak hydrogen concentration is $9,800 \mathrm{ppm}$, compared with $7,500 \mathrm{ppm}$ in the base case, and the $95 \%$ confidence limit is 17,100 ppm, exceeding $25 \%$ LFL by $70 \%$ (15,100 ppm in the base case).

As in the base case, 1,300 of the 10,000 simulations had no BDRGEs. This may be seen in the distribution for the fraction of the retained gas released by BDGREs during the simulations (Figure 4.30). The median release fraction is 0.09 , and the $95 \%$ confidence level is 0.23 , equivalent to the base case results.

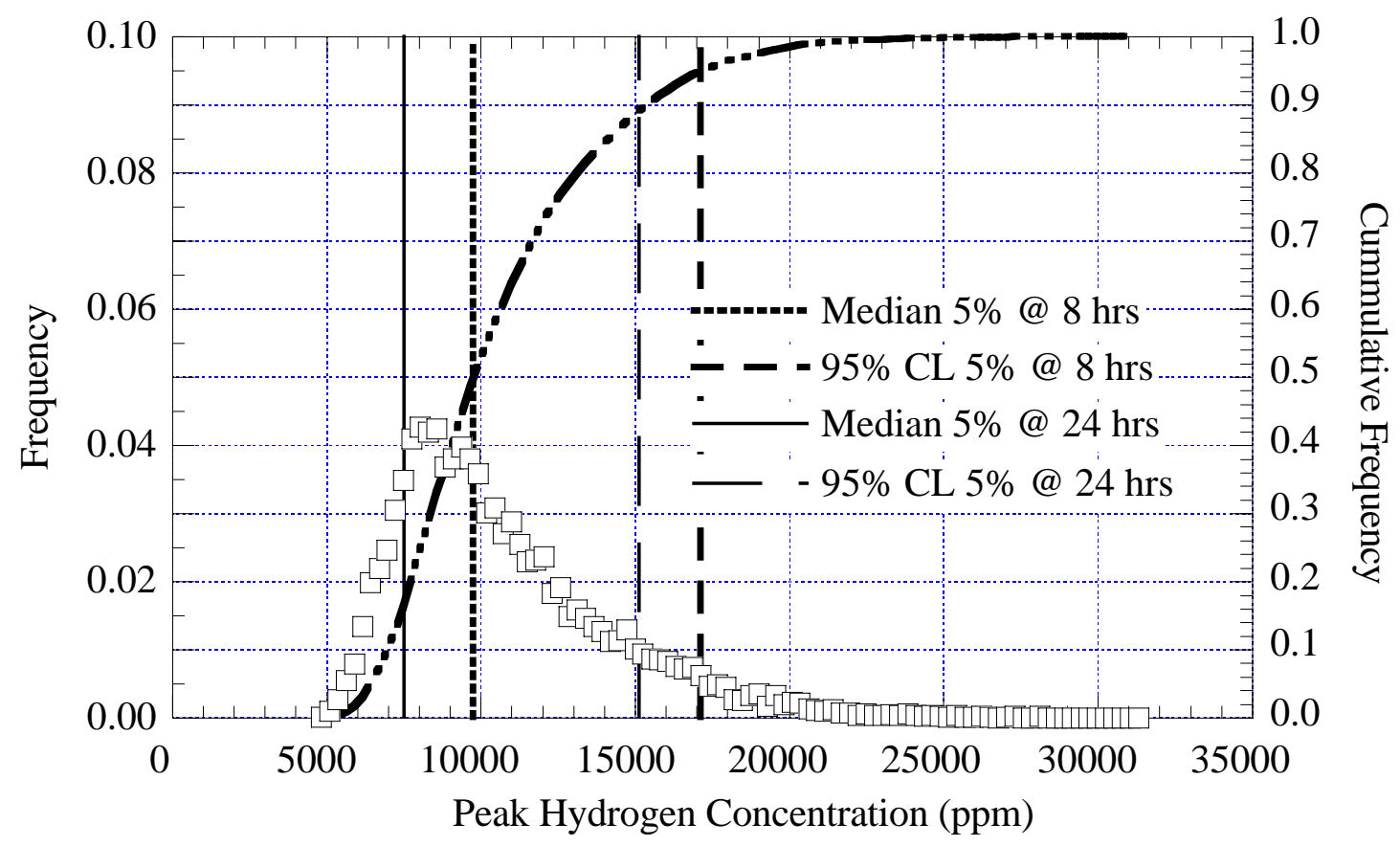

Figure 4.29. Peak Hydrogen Concentration: 5\% Disturbance Every 8 Hours in AN-105 


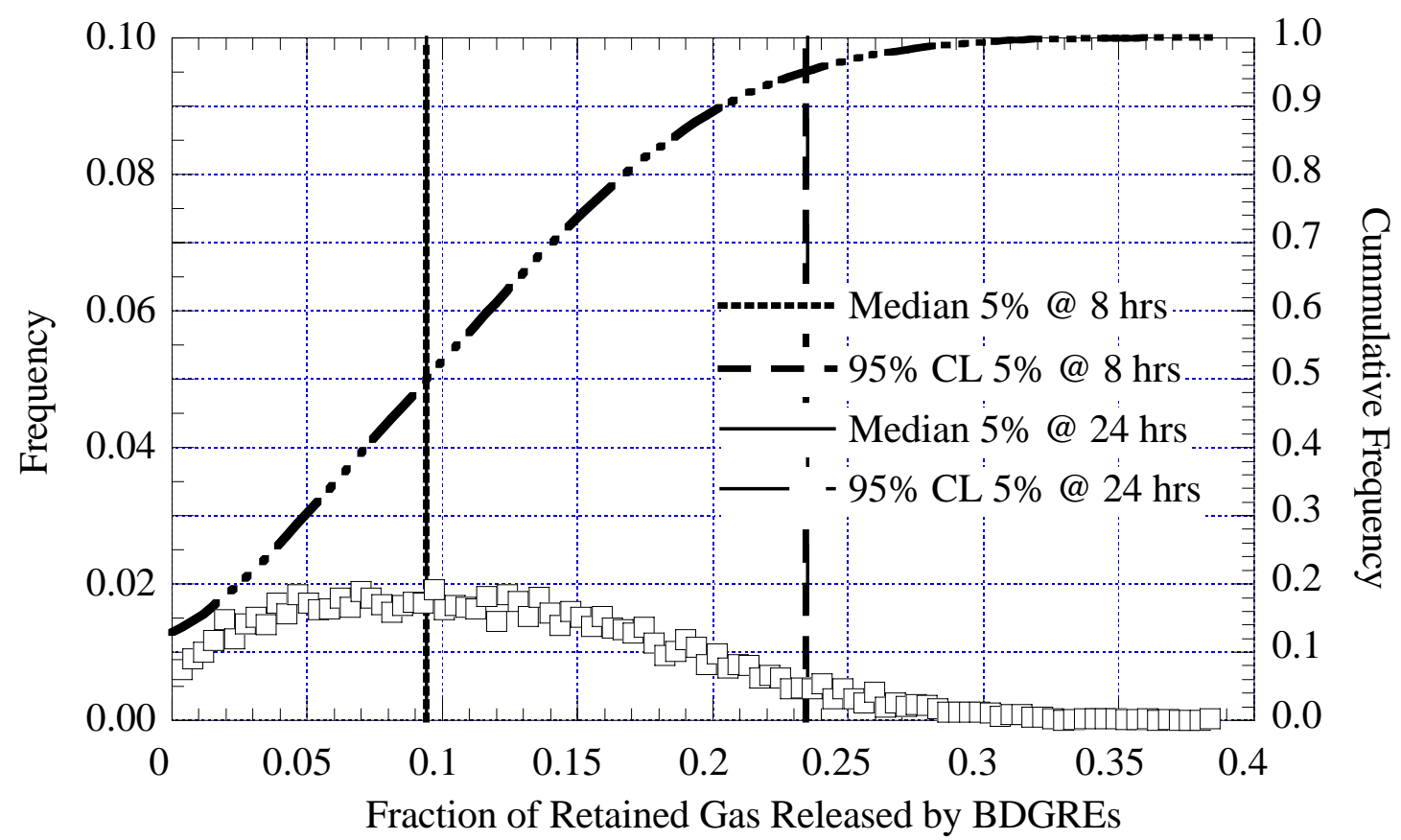

Figure 4.30. Gas Released by BDGREs: 5\% Disturbance Every 8 Hours in AN-105

The headspace hydrogen concentration for a simulation representing the $95 \%$ confidence level peak hydrogen concentration is plotted in Figure 4.31 (same simulation as base case). Again, the repeated pump runs can be seen in spikes every eight hours, and the highest headspace hydrogen concentrations are due to spontaneous BDGREs. Two BDGREs are triggered immediately after the fourth pump run at 24 hours, and the final BDGRE is triggered on the next to the last pump run at 128 hours. The gas volume distribution in the gobs affected by the subsequent pump runs is again directly evident in the varied concentration results over the pump runs (see Section 4.2). The total mixing time is approximately 140 hours.

The average hydrogen concentration in Figure 4.31 is greater than that in Figure 4.25, suggesting the potential for increased risk with a higher initial concentration. As illustrated in Figure 4.29 however, this effect does not have significant impact on the $95 \%$ confidence level of the peak hydrogen concentration.

The results show that for 5\% of the sediment layer disturbed by mixer pump operations every eight hours, it is highly likely in the 95\% confidence level that the hydrogen concentration will exceed $25 \%$ of the LFL. However, it is highly unlikely it will reach the LFL. Lengthening the time between pump runs to 24 hours, as in the base case, clearly allows more time for hydrogen in the headspace to dissipate, but this does not appear to reduce the peak concentrations significantly. 


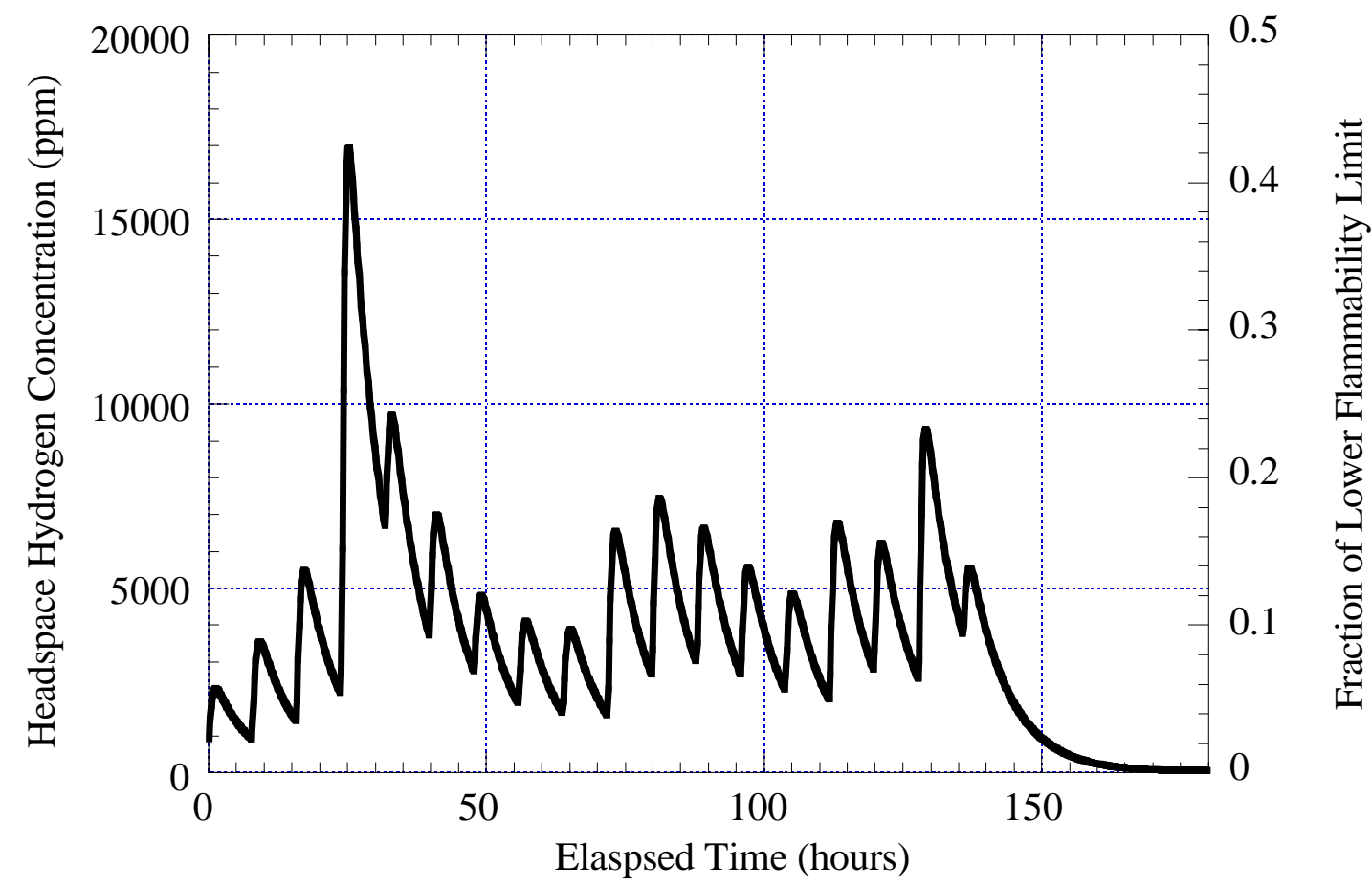

Figure 4.31. Example Simulation Hydrogen Concentration: 5\% Disturbance Every 8 Hours in AN-105

\subsubsection{Summary of Mixer Pump Analyses for AN-105}

The mixing results for AN-105 are summarized in Table 4.6. The gas releases due to mixer pump operation and induced BDGREs are not likely to approach flammability as long as pump operations are carefully controlled. The results indicate that pump runs that disturb on the order of $5 \%$ of the sediment layer repeated every 24 hours provide an acceptable margin between the peak headspace hydrogen concentration and the flammability limit. Increasing the pump speed and/or duration to disturb $20 \%$ of the waste produces hydrogen concentrations approaching within almost $75 \%$ of the LFL. Decreasing the waiting time between runs to eight hours increases the peak hydrogen concentration by $13 \%$.

Table 4.6. Summary of Mixer Pump Analysis Results in AN-105

\begin{tabular}{||l|l|l|c|c||}
\hline Tank & Run & Quantity & Median & 95\% CL \\
\hline AN-105 & $5 \%$ at 24 hours & Hydrogen $(\mathrm{ppm})$ & 7,500 & 15,100 \\
\hline & & Fraction Gas Release by BDGREs & 0.09 & 0.23 \\
\hline & $20 \%$ at 24 hours & Hydrogen $(\mathrm{ppm})$ & 20,300 & 28,400 \\
\hline & Fraction Gas Release by BDGREs & 0.08 & 0.22 \\
\hline & $5 \%$ at 8 hours & Hydrogen $(\mathrm{ppm})$ & 9,800 & 17,100 \\
\hline & Fraction Gas Release by BDGREs & 0.09 & 0.23 \\
\hline
\end{tabular}




\subsection{Analysis of Mixing-Induced Gas Releases in Other Tanks}

Mixing-induced gas releases in the three other tanks were analyzed. The initial conditions for each tank were determined from historical data and measurements the same way as AN-105, as described in Sections 3.2 and 4.3. Based on the results of the decant analysis (Sections 3.3. to 3.5) and the mixing results in AN-105 (Section 4.4), only select cases were run in each tank, as discussed below. The mixing results for all of the tanks are summarized in Section 4.6.

\subsubsection{AN-103 Mixing Results}

As discussed and as reflected in the decant results (Sections 3.3 to 3.5), AN-103 contains by far the highest retained gas volume of the four tanks considered in this study. Therefore, the base case (5\% disturbance every 24 hours) was the only case run in AN-103 because this produced the lowest headspace hydrogen concentration in AN-105 (Section 4.4).

The distribution of the peak hydrogen concentration for the base case is presented in Figure 4.32. The median of the peak hydrogen concentration is $15,300 \mathrm{ppm}$, and the $95 \%$ confidence limit is $25,800 \mathrm{ppm}$. Only 58 of the 10,000 simulations had no induced BDGREs. The distribution for the fraction of the retained gas released by BDGREs during the simulations is shown in Figure 4.33. The median release fraction is 0.20 and the $95 \%$ confidence level 0.33 .

The results show that for 5\% of the sediment layer disturbed by mixer pump operations every 24 hours, it is highly likely in the $95 \%$ confidence level that the hydrogen concentration will exceed $50 \%$ of the LFL, and 10 out the 10,000 simulations exceeded LFL.

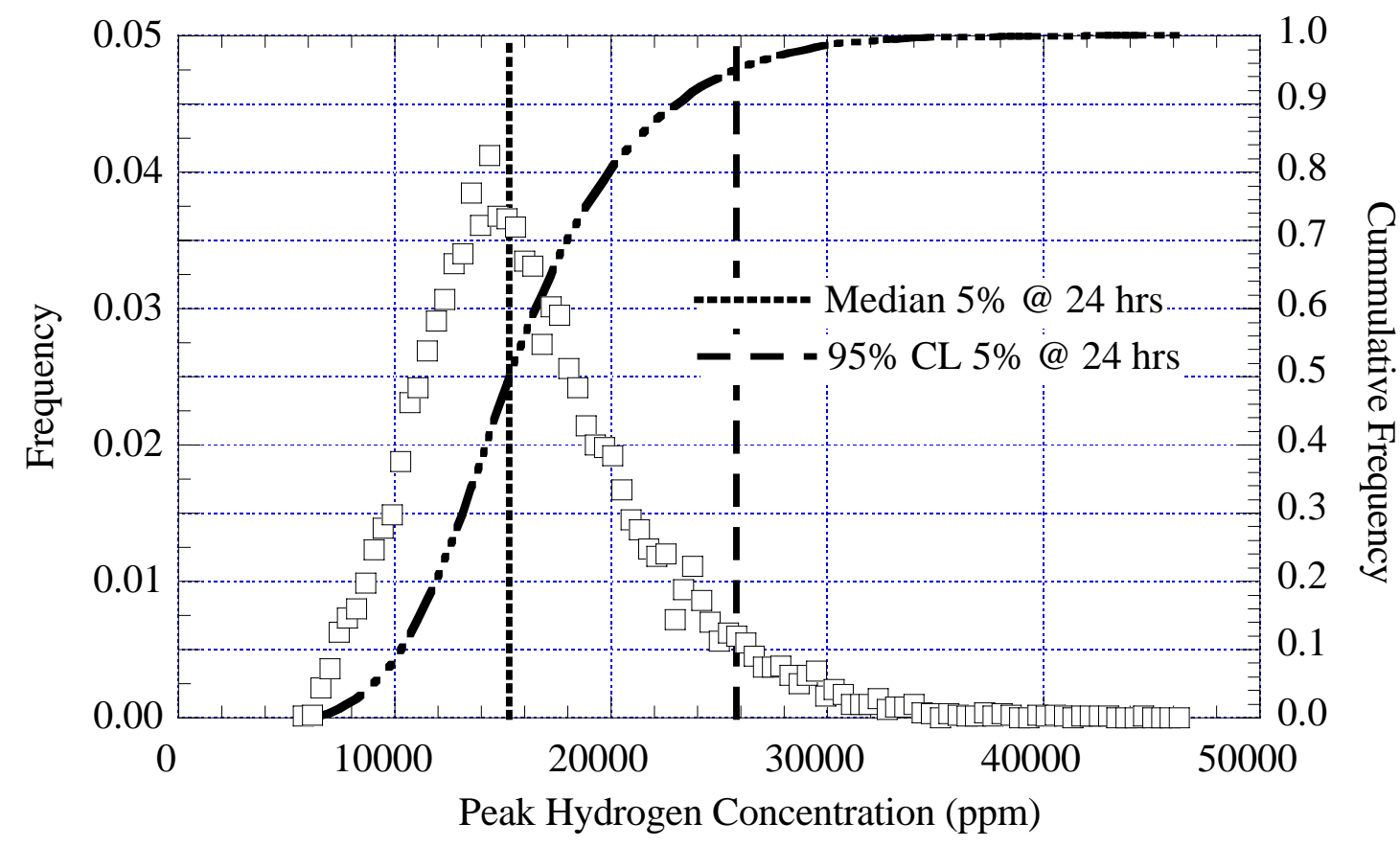

Figure 4.32. Peak Hydrogen Concentration: 5\% Disturbance Every 24 Hours in AN-103 


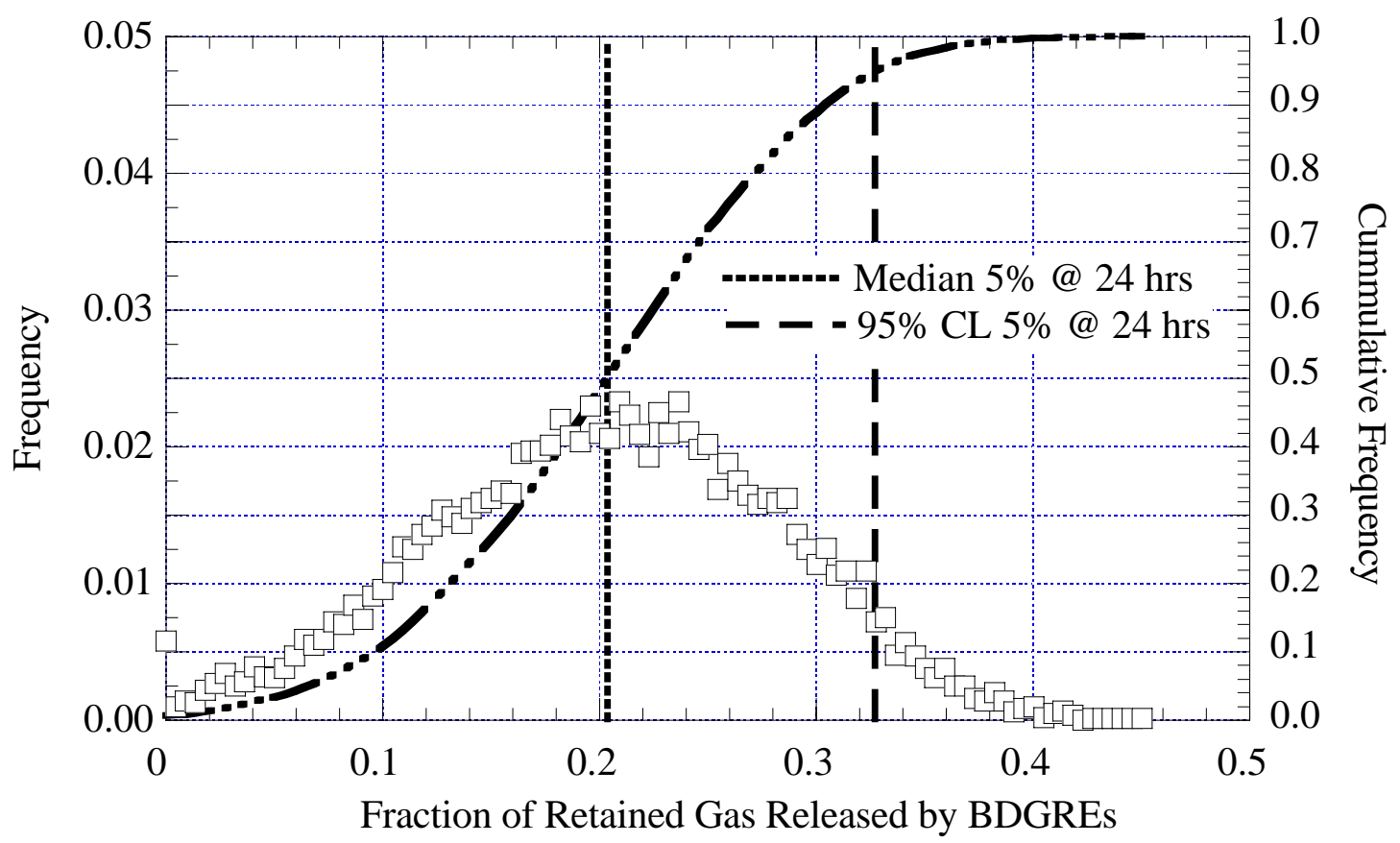

Figure 4.33. Gas Released by BDGREs: 5\% Disturbance Every 24 Hours in AN-103

\subsubsection{AN-104 Mixing Results}

Tank AN-104 has a larger retained gas volume and a larger headspace than AN-105 and exhibited behavior similar to AN-105 in the decant analysis (Sections 3.3 through 3.5). Thus it is expected that effects of the accelerated pump schedule (pump run every eight hours) will be similar, so only the base case (5\% disturbance every 24 hours) and the $20 \%$ disturbance every 24 hours were analyzed in AN-104.

The distributions of the peak hydrogen concentration and BDGRE gas release fraction for the base case are given in Figures 4.34 and 4.35, respectively. The median of the peak hydrogen concentration is $5,500 \mathrm{ppm}$ and the $95 \%$ confidence limit $10,600 \mathrm{ppm}$, slightly exceeding $25 \%$ LFL. The median BDGRE release fraction is 0.12 , the $95 \%$ confidence level is 0.26 , and 846 of the 10,000 simulations had no induced BDGREs.

As with AN-105, the $20 \%$ disturbance every 24 hours peak hydrogen concentration results are higher than the base-case results (Figure 4.36). The median is 12,900 ppm ( $32 \%$ LFL), and the $95 \%$ confidence level is $18,500 \mathrm{ppm}(\sim 46 \% \mathrm{LFL})$. The distribution for the fraction of the retained gas released by BDGREs during the simulations is shown in Figure 4.37. The median fraction of the retained gas released by BDGREs is 0.10 and the $95 \%$ confidence level is 0.25 , with 1,233 of the simulations having no induced BDRGEs. 


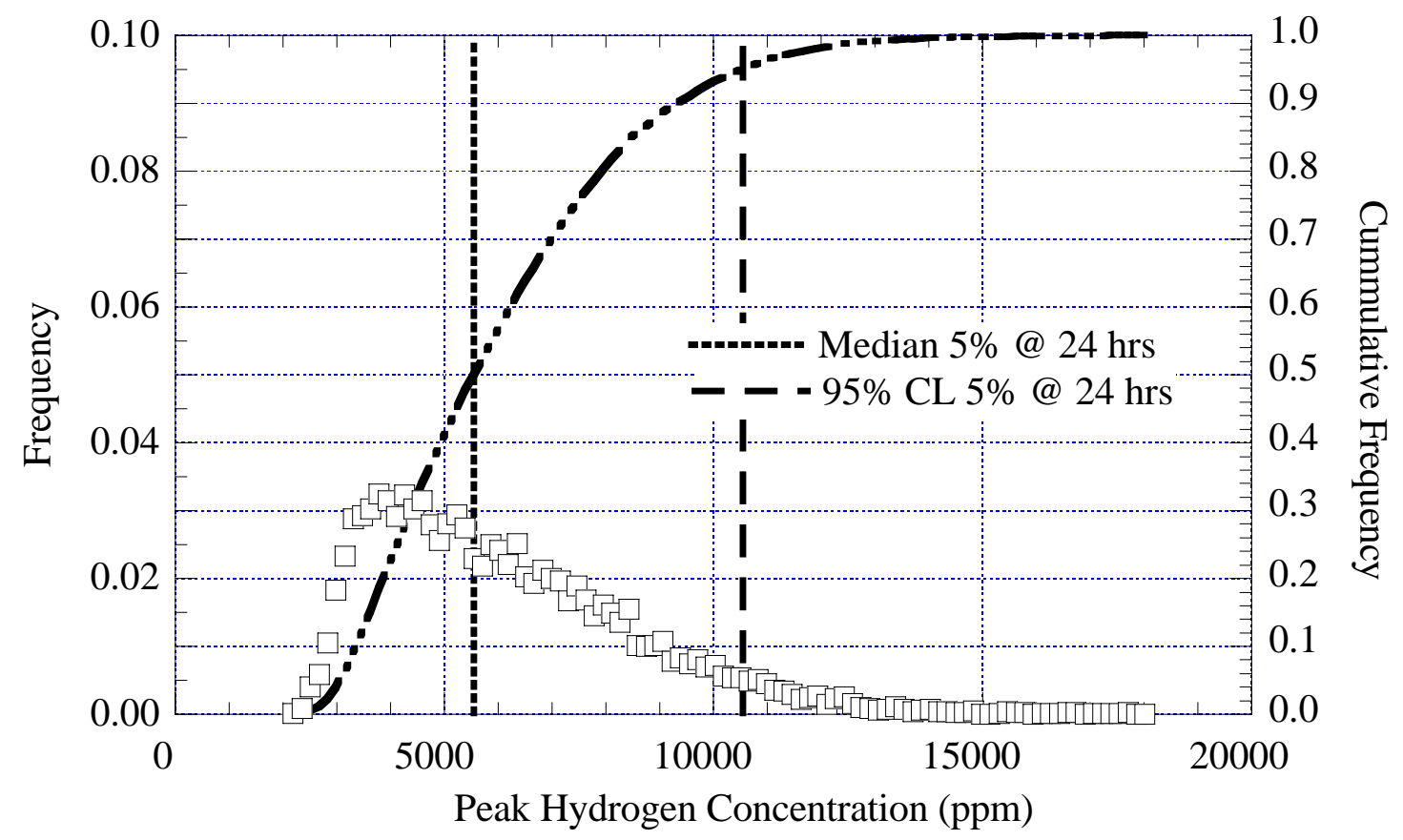

Figure 4.34. Peak Hydrogen Concentration: 5\% Disturbance Every 24 Hours in AN-104

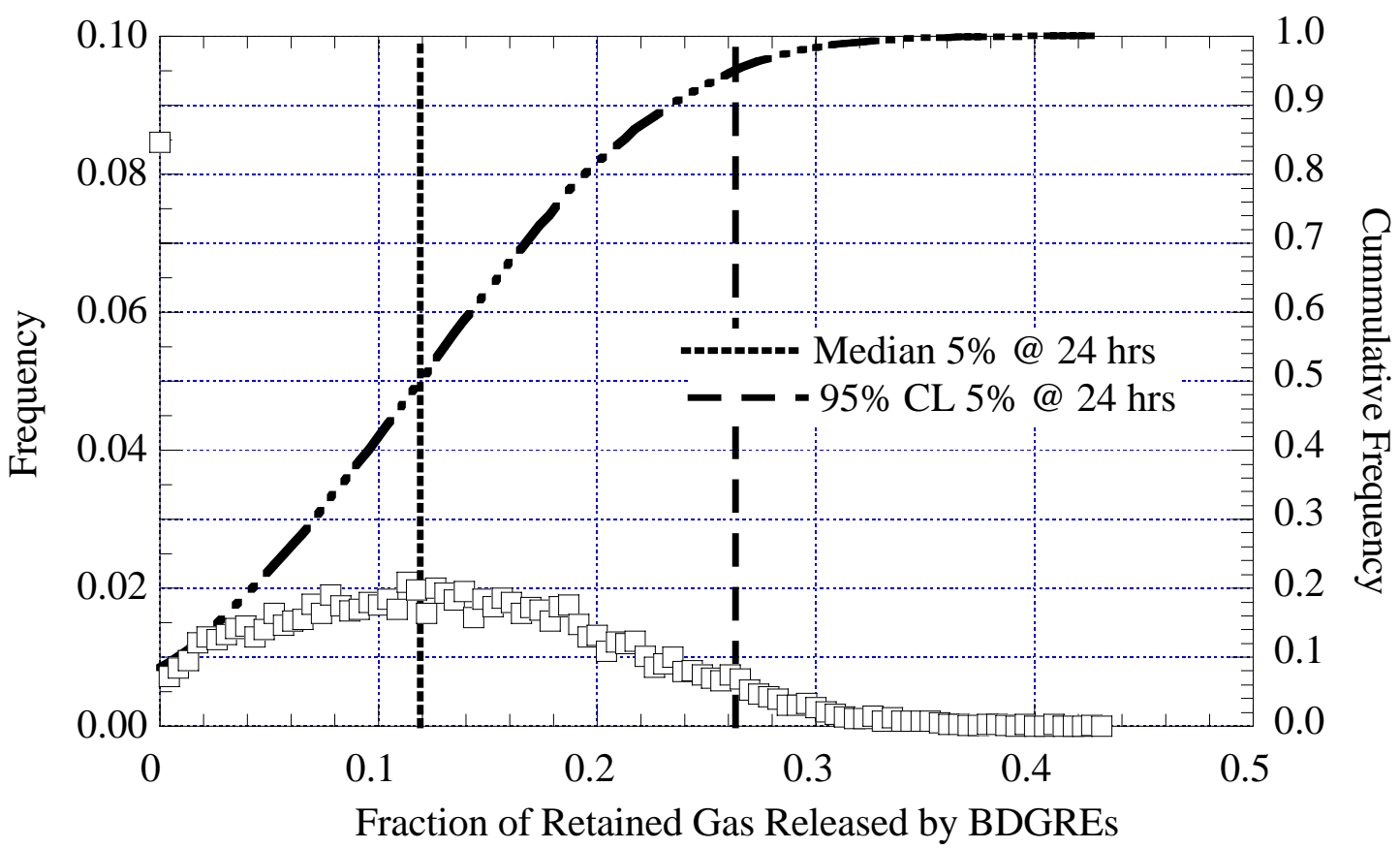

Figure 4.35. Gas Released by BDGREs: 5\% Disturbance Every 24 Hours in AN-104 


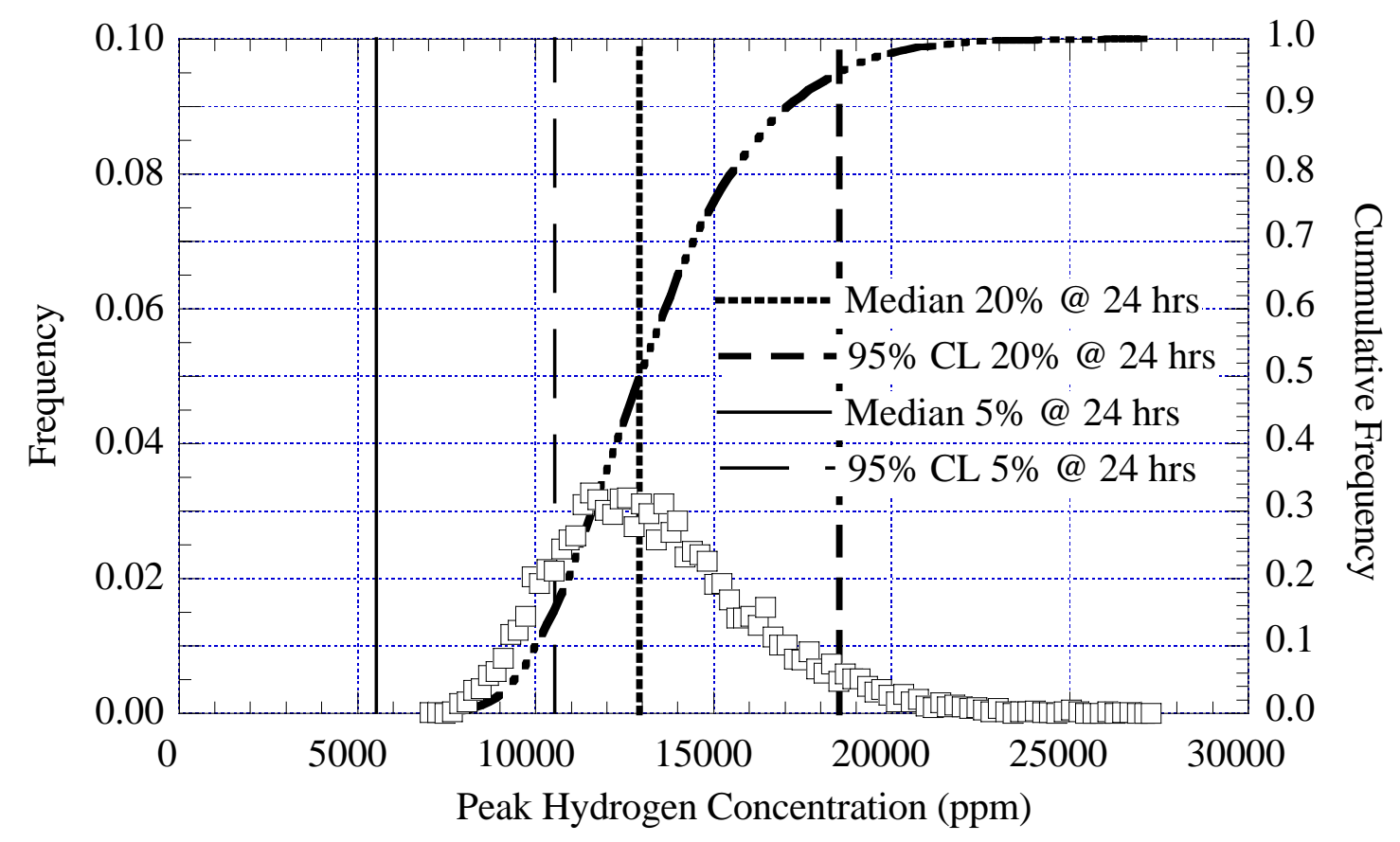

Figure 4.36. Peak Hydrogen Concentration: $20 \%$ Disturbance Every 24 Hours in AN-104

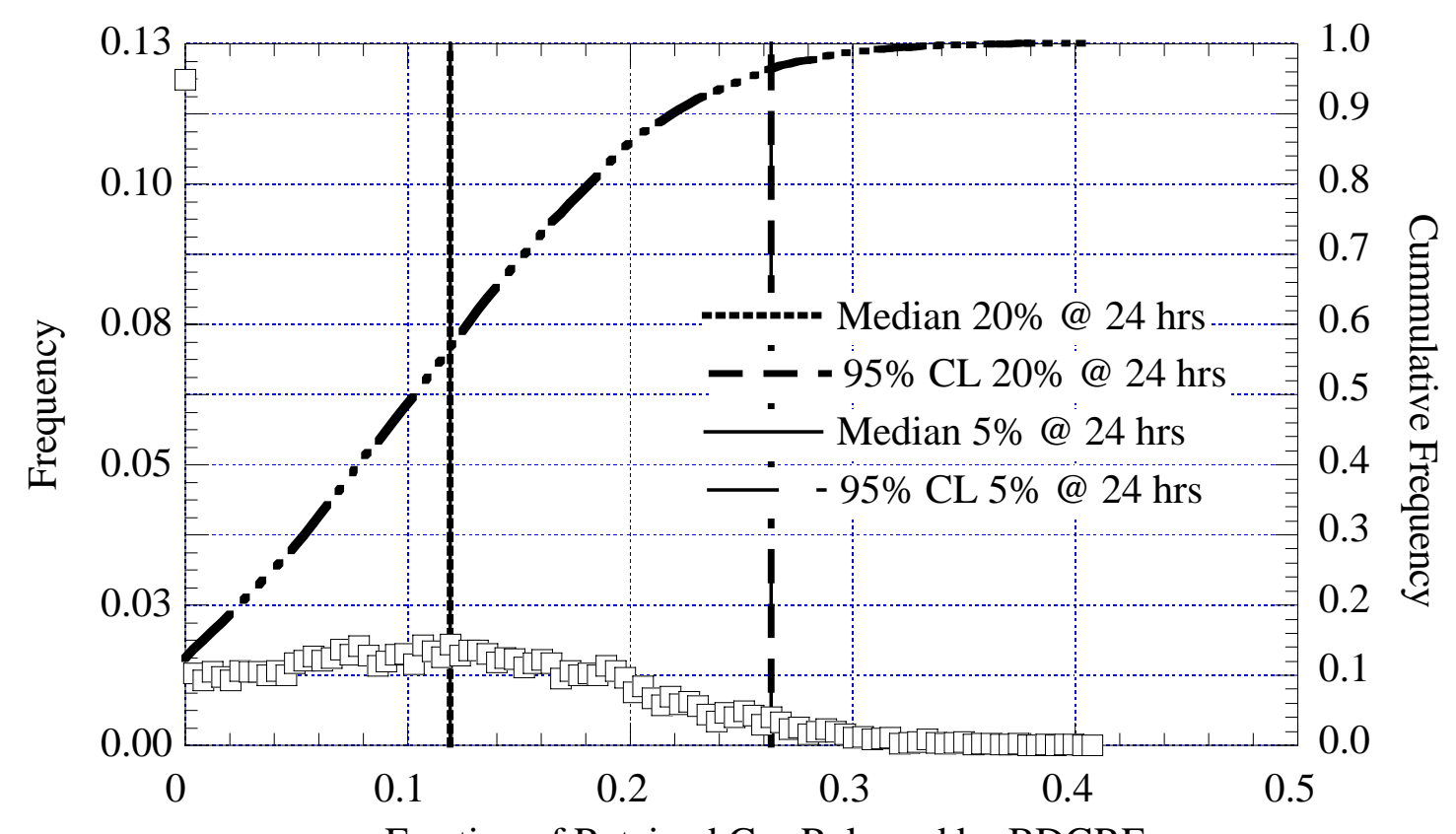

Figure 4.37. Gas Released by BDGREs: 20\% Disturbance Every 24 Hours in AN-104 


\subsubsection{AW-101 Mixing Results}

Tank AW-101 is notable for having the least retained gas volume of the four tanks under consideration. This was reflected in the decant results in Sections 3.4 and 3.5. Therefore, in addition to the base case, a 20\% disturbance every eight hours case was also evaluated.

The distributions of peak hydrogen concentration and gas release fraction for the base case are provided in Figures 4.38 and 4.39, respectively. The median and $95^{\text {th }}$ percentile values of the peak hydrogen concentration are only 1,800 ppm ( 5\% LFL) and 2,700 ppm ( 7\% LFL), respectively. The fraction of the retained gas released by BDGREs is 0.00 at the median and 0.14 at the $95^{\text {th }}$ percentile, with over $64 \%$ of the simulations having no BDGREs.

The $20 \%$ disturbance every eight hours peak hydrogen concentration and the fraction of the retained gas released by BDGREs results are shown in Figures 4.40 and 4.41, respectively. The median peak hydrogen concentration is $6,900 \mathrm{ppm}$, and the $95 \%$ confidence level is still below $25 \% \mathrm{LFL}$ at $8,800 \mathrm{ppm}$. The median fraction of the retained gas released by BDGREs is 0.00 , and the $95 \%$ confidence level is 0.13 . Almost $70 \%$ of the simulations had no induced BDGREs. These results indicate that in AW-101, even for the maximum feasible mixing schedule and operations, the LFL will not be threatened.

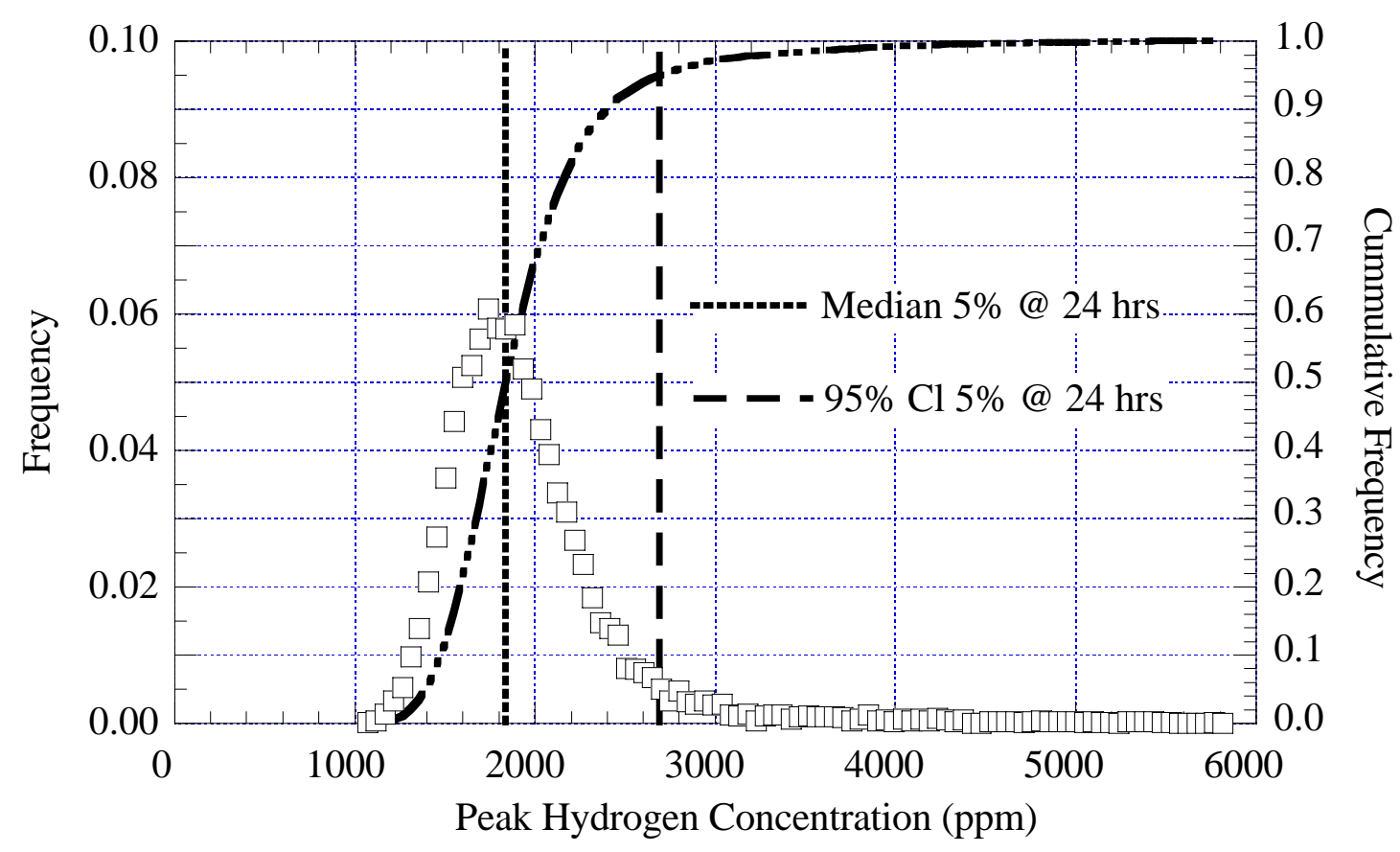

Figure 4.38. Peak Hydrogen Concentration: 5\% Disturbance Every 24 Hours in AW-101 


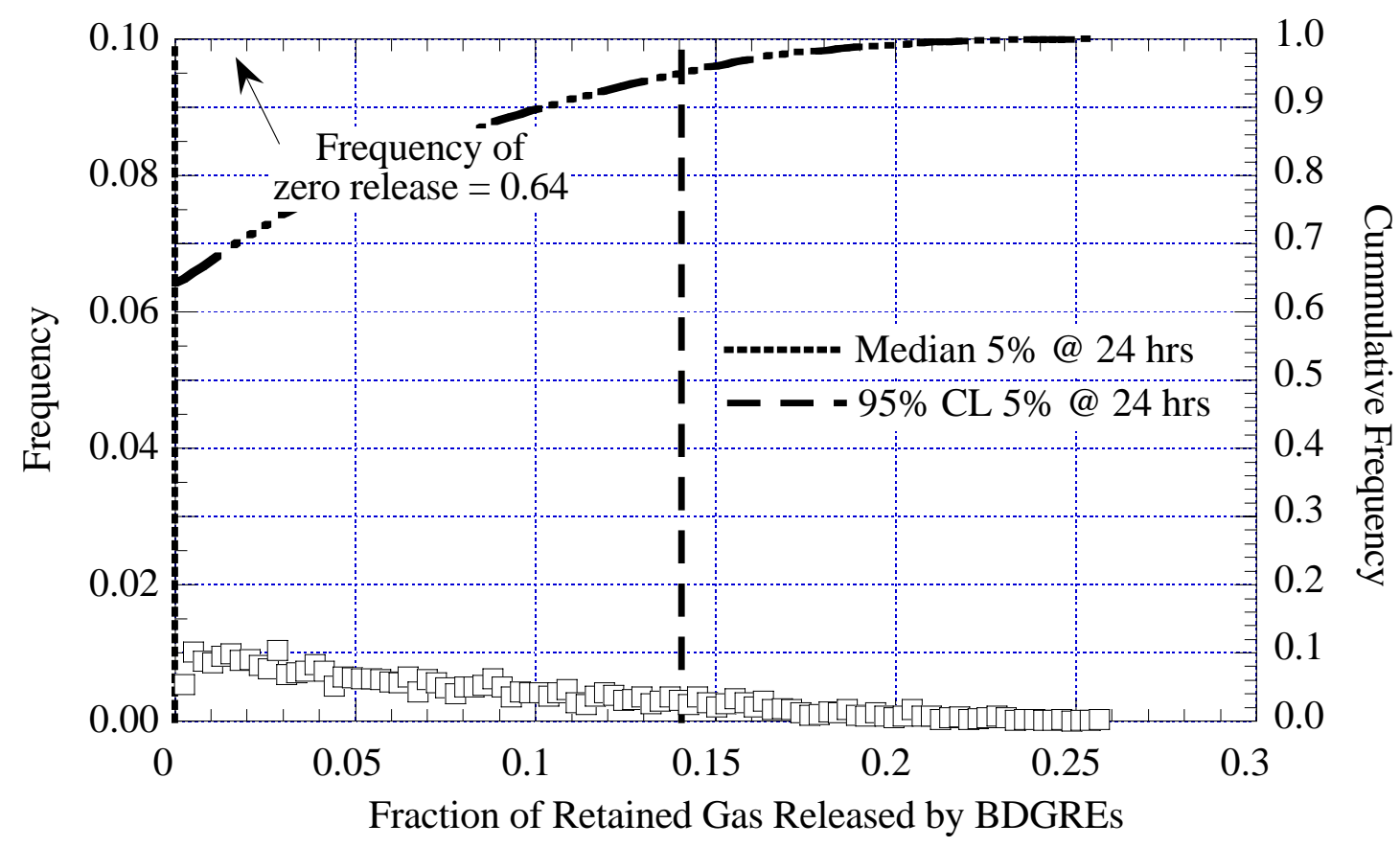

Figure 4.39. Gas Released by BDGREs: 5\% Disturbance Every 24 Hours in AW-101

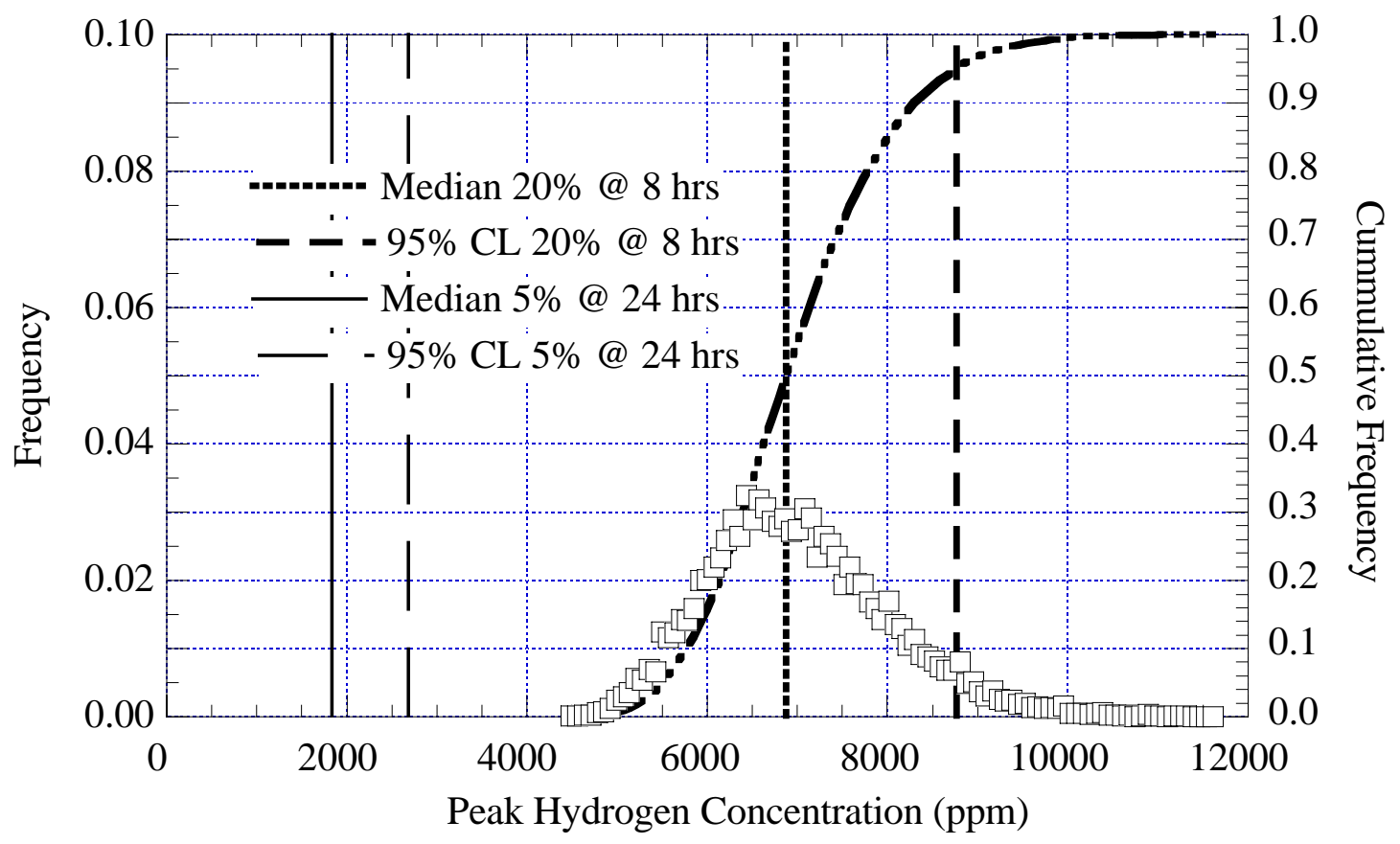

Figure 4.40. Peak Hydrogen Concentration: 20\% Disturbance Every 8 Hours in AW-101 


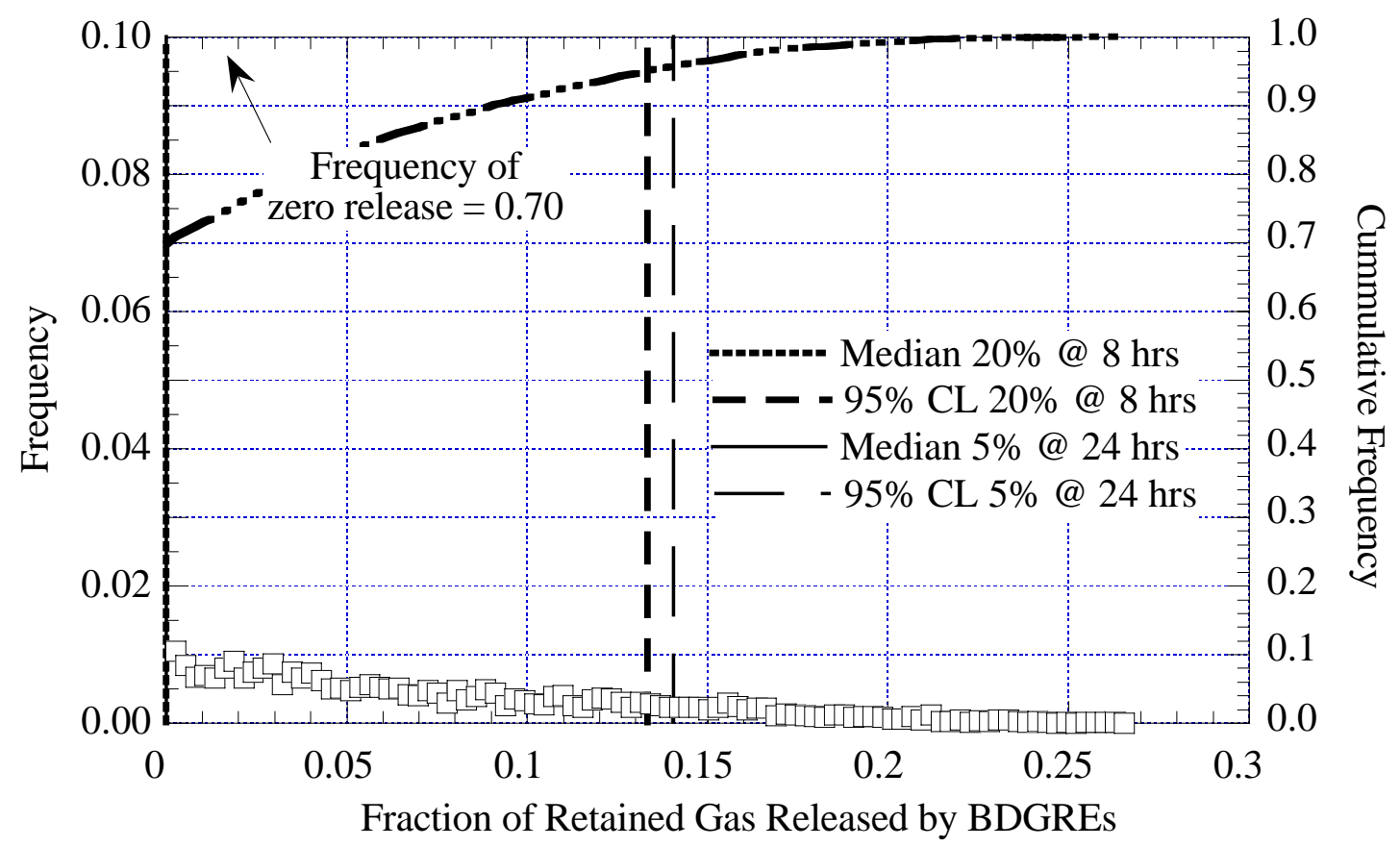

Figure 4.41. Gas Released by BDGREs: 20\% Disturbance Every 8 Hours in AW-101

\subsection{Conclusions from Mixing-Induced Gas Release Modeling Results}

The mixing results are summarized in Table 4.7. Except for AW-101, the base case (5\% disturbance every 24 hours) had results at the 95\% confidence level that exceeded $25 \%$ LFL. However, none of the tanks exceeded LFL at the 95\% confidence level. Increasing the volume of waste disturbed produces elevated hydrogen concentrations. To a lesser extent, decreasing the waiting time between pump runs also elevates the peak hydrogen concentrations. The peak hydrogen concentrations during mixing operations are caused by induced BDGREs. 
Table 4.7. Summary of Mixing Analysis Results

\begin{tabular}{|c|c|c|c|c|}
\hline Tank & Run & Quantity & Median & $95 \% C L^{(a}$ \\
\hline \multirow[t]{6}{*}{ AN-105 } & $5 \%$ at 24 hours & Hydrogen (ppm) & 7,500 & 15,100 \\
\hline & & Fraction Gas Release by BDGREs & 0.09 & 0.23 \\
\hline & $20 \%$ at 24 hours & Hydrogen (ppm) & 20,300 & 28,400 \\
\hline & & Fraction Gas Release by BDGREs & 0.08 & 0.22 \\
\hline & $5 \%$ at 8 hours & Hydrogen (ppm) & 9,800 & 17,100 \\
\hline & & Fraction Gas Release by BDGREs & 0.09 & 0.23 \\
\hline \multirow[t]{4}{*}{ AN-104 } & $5 \%$ at 24 hours & Hydrogen (ppm) & 5,500 & 10,600 \\
\hline & & Fraction Gas Release by BDGREs & 0.12 & 0.26 \\
\hline & $20 \%$ at 24 hours & Hydrogen (ppm) & 12,900 & 18,500 \\
\hline & & Fraction Gas Release by BDGREs & 0.10 & 0.25 \\
\hline \multirow{2}{*}{$\mathrm{AN}-103$} & $5 \%$ at 24 hours & Hydrogen (ppm) & 15,300 & 26,000 \\
\hline & & Fraction Gas Release by BDGREs & 0.20 & 0.33 \\
\hline \multirow[t]{4}{*}{ AW-101 } & $5 \%$ at 24 hours & Hydrogen (ppm) & 1,800 & 2,700 \\
\hline & & Fraction Gas Release by BDGREs & 0.00 & 0.14 \\
\hline & $20 \%$ at 8 hours & Hydrogen (ppm) & 6,900 & 8,800 \\
\hline & & Fraction Gas Release by BDGREs & 0.00 & 0.13 \\
\hline
\end{tabular}




\subsection{Conclusions and Recommendations}

A thorough review was conducted of buoyant displacement gas release mechanisms and how they can be induced and exacerbated by supernate decanting and mixer pump operation. A detailed analysis of historic gas release data has provided a much better understanding of tank behavior, and new methods have been developed for quantifying the jet mixing process. The result of these efforts is a new, robust model to predict the headspace hydrogen concentration during various retrieval operations. The derivation of this model and its application to supernate decanting and mixer pump degassing in Tanks AN-103, AN-104, AN-105 and AW-101 are the subject of this report. Overall conclusions from the analyses are given in Section 5.1 and recommendations in Section 5.2.

\subsection{Conclusions}

Except for AN-103, the peak hydrogen concentrations resulting from gas releases predicted to be induced by supernate decant roughly correspond to those produced by the larger spontaneous GREs and remain well below the LFL. AN-103 is an exception because its history of very small, infrequent gas releases does not match the expectation based on its waste configuration. Nevertheless, none of the runs for AN-103 reached the LFL, even though the majority of the runs had BDGREs occur relatively early in the decant. AW-101 lies at the other extreme in that its predicted peak hydrogen concentrations are far below even the 6,250-ppm action level. These analysis results indicate that removing the supernatant liquid from these four tanks will not induce gas releases that cause the tank headspace to reach the LFL.

These results are not sensitive to the decant rate, and the predicted peak hydrogen concentration is not reduced by stopping the decant at the action level of 6,250 ppm hydrogen. However, backfilling with water during the decant has a significant probability of preventing BDGREs, though the peak hydrogen concentration approximates the base case if they do occur (excepting AW-101).

The gas releases that occur during mixer pump degassing are not likely to approach flammability as long as the pump operations are carefully controlled. The modeling results indicate that pump runs that disturb on the order of 5\% of the sediment layer repeated every 24 hours provide an acceptable margin between the peak headspace hydrogen concentration and the LFL. Increasing the pump speed or duration to disturb $20 \%$ of the waste produces significantly elevated hydrogen concentrations. Decreasing the waiting time between runs to 8 hours elevates the peak hydrogen concentration to a lesser extent. Based on the jet mixing model developed in this study, operating one W-211 mixer pump at fixed azimuth for 26 minutes at 1,200 rpm (full power) or 48 minutes at $700 \mathrm{rpm}$ (idle) would disturb approximately $5 \%$ of the waste. A $20 \%$ waste disturbance would require 76 minutes at 1,200 rpm. 


\subsection{Recommendations}

Development of the jet-mixing model was hindered by the lack of good data on the transient mobilization of sediment and corresponding gas release. The only available data on gas release were from SY-101 and were complicated by the high uncertainty in the retained gas volume and the complexity of the mixer pump operations. While the model correlates these data very well, the extrapolation to the larger W-211 pumps with their different design and location in the tank is quite uncertain. The following two activities could greatly reduce the technical risk of waste feed delivery:

- Additional data for model validation should be developed from lab-scale experiments with jets in simulated gas-retaining sediment layers. Because we are looking primarily for first-order behavior, many of the technical issues associated with smaller scale and with developing representative waste simulants would not be of concern.

- Computational fluid dynamics simulations (TEMPEST; Onishi and Trent 1998) should be performed with the goal of looking at the penetration and collapse mechanisms and the effects of gradients in material strength. Information from these simulations would provide unique and valuable insights into the actual processes involved.

We believe that the modeling results show that decanting and mixing retrieval operations have a conservative margin with regard to flammable conditions and, with reasonable care, can be safely conducted in the tanks we analyzed. However, the most conservative approach to remove the uncertainty inherent in these analytical model results would be to perform decant or mixing operations in a tank that does not have sufficient gas to render the headspace flammable even if it were all released instantaneously. Tanks AW-101 and SY-103 meet this criterion. 


\subsection{References}

Alleman RT, ZI Antoniak, WD Chavala, LE Efferding, JG Fadeff, JR Friley, WB Gregory, JD Hudson, JJ Irwin, NW Kirch, TE Michener, FE Panisko, CW Stewart, and BM Wise. 1994. Mitigation of Tank 241-SY-101 by Pump Mixing: Results of Testing Phases A and B. PNL-9423, Pacific Northwest Laboratory, Richland, Washington.

Brewster ME, NB Gallagher, JD Hudson, and CW Stewart. 1995. The Behavior, Quantity, and Location of Undissolved Gas in Tank 241-SY-101. PNL-10681, Pacific Northwest Laboratory, Richland, Washington.

Caley SM, CW Stewart, ZI Antoniak, JM Cuta, LA Mahoney, FE Panisko. 1998. Investigation of Flammable Gas and Thermal Safety Issues for Retrieval of Waste from Tank 241-AN-105. PNNL-11996. Pacific Northwest National Laboratory, Richland, Washington.

CHG. 2000. Tank Waste Remediation System Technical Safety Requirements, Rev. 2 as amended. HNF-SD-WM-TSR-006, CH2M HILL Hanford Group, Richland, Washington.

Hedengren DC, KM Hodgson, WB Barton, CW Stewart, JM Cuta, and BE Wells. 2000. Data Observations on Double-Shell Flammable Gas Watch List Tank Behavior. RPP-6655. Rev. 0. CH2M HILL Hanford Group, Inc., Richland, Washington.

Hu TA. 2000. Empirical Rate Equation Model and Rate Calculations for Hydrogen Generation for Hanford Tank Waste. HNF-3851 Rev. 0A, CH2M HILL Hanford Group, Inc., Richland, Washington.

Huckaby JL, JC Hayes, L Jensen, LL Buckley, RD Cromar, LD Pennington, and SR Wilmarth. 1997. Homogeneity of Passively Ventilated Waste Tanks. PNNL-11640, Pacific Northwest National Laboratory, Richland, Washington.

Mahoney LA, JL Huckaby, SA Bryan, and GD Johnson. 2000. Overview of the Flammability of Gases Generated in Hanford Waste Tanks. PNNL-13269, Pacific Northwest National Laboratory, Richland, Washington.

Mahoney LA, ZI Antoniak, JM Bates, and ME Dahl. 1999. Retained Gas Sampling Results for the Flammable Gas Program. PNNL-13000, Pacific Northwest National Laboratory, Richland, Washington.

McCain DJ. 2001. Results of Vapor Space Monitoring of Flammable Gas Watch List Tanks. HNF-SD-WM-TI-797 Rev. 6, CH2M HILL Hanford Group, Inc., Richland, Washington.

Meyer PA and CW Stewart. 2001. Preventing Buoyant Displacement Gas Release Events in Hanford Double-Shell Waste Tanks. PNNL-13337, Pacific Northwest National Laboratory, Richland, Washington. 
Meyer PA, ME Brewster, SA Bryan, G Chen, LR Pederson, CW Stewart, G Terrones. 1997. Gas Retention and Release Behavior in Double-Shell Waste Tanks. PNNL-11536 Rev. 1, Pacific Northwest National Laboratory, Richland, Washington.

Moore DJ, SA Barker, WB Barton, and MA Kufahl. 2000. The Gas Release Event Safety Analysis Tool Relational Data Base for Hanford Tanks. HNF-SD-WM-TI-806 Rev. 3, CH2M HILL Hanford Group, Inc., Richland, Washington.

Onishi Y and DS Trent. 1998. TEMPEST Code Modifications and Testing for Erosion-Resisting Sludge Simulations. PNNL-11787, Pacific Northwest National Laboratory, Richland, Washington.

Reynolds DA. 1993. Tank 101-SY Window E Core Sample: Interpretation of Results. WHC-EP0628, Westinghouse Hanford Company, Richland, Washington.

Rajaratnam N. 1976. Turbulent Jets. American Elsevier Publishing Company, Inc., New York.

Stewart CW, ME Brewster, PA Gauglitz, LA Mahoney, PA Meyer, KP Recknagle, and HC Reid. 1996. Gas Retention and Release Behavior in Hanford Single-Shell Waste Tanks. PNNL-11391, Pacific Northwest National Laboratory, Richland, Washington.

Sullivan HL. 1995. A Safety Assessment for Proposed Pump Mixing Operations to Mitigate Episodic Gas Releases in Tank 241-SY-101: Hanford Site, Richland, Washington. LA-UR-923196 Rev. 14, Los Alamos National Laboratory, Los Alamos, New Mexico. 